FACULDADE DE FILOSOFIA, CIÊNCIAS E LETRAS DE RIBEIRÃO PRETO PROGRAMA DE PÓS-GRADUAÇÃO EM ENTOMOLOGIA

\title{
A comparative morphology of Oscinellinae genera (Diptera: \\ Chloropidae): a framework towards a phylogeny of the subfamily
}

\begin{abstract}
"Morfologia comparada dos gêneros de Oscinellinae (Diptera: Chloropidae): estrutura para uma filogenia da subfamília"
\end{abstract}

\section{Zeinab Bazyar}

Thesis presented to the Faculty of Philosophy, Sciences and Letters of Ribeirão Preto of the University of São Paulo, part of the requirements for obtaining the title of Doctor of Philosophy (Ph.D), obtained in the Postgraduate Program in Entomology.

Ribeirão Preto - SP 
UNIVERSIDADE DE SÃO PAULO

FACULDADE DE FILOSOFIA, CIÊNCIAS E LETRAS DE RIBEIRÃO PRETO PROGRAMA DE PÓS-GRADUAÇÃO EM ENTOMOLOGIA

A comparative morphology of Oscinellinae genera (Diptera: Chloropidae): a framework towards a phylogeny of the subfamily

"Morfologia comparada dos gêneros de Oscinellinae (Diptera: Chloropidae):
estrutura para uma filogenia da subfamília"

Zeinab Bazyar

Advisor: Dalton de Souza Amorim

Thesis presented to the Faculty of Philosophy, Sciences and Letters of Ribeirão Preto of the University of São Paulo, part of the requirements for obtaining the title of Doctor of Philosophy (Ph.D), obtained in the Postgraduate Program in Entomology.

Ribeirão Preto - SP 
I authorize the reproduction and total or partial disclosure of this work, by any conventional or electronic means, for study and research purposes, provided the source is mentioned.

Bazyar, Zeinab

A comparative morphology of Oscinellinae genera (Diptera: Chloropidae): a framework towards a phylogeny of the subfamily / Zeinab Bazyar - 2019.

161 pages

Doctoral thesis presented at Faculdade de Filosofia, Ciências e Letras de Ribeirão Preto, University of São Paulo. Area of concentration: Entomology.

Advisor: Dalton de Souza Amorim

1. Acalyptratae. 2. Chloropidae. 3. Diptera. 4. Phylogeny. 5. Oscinellinae. 6. Worldwide. 


\section{Contents}

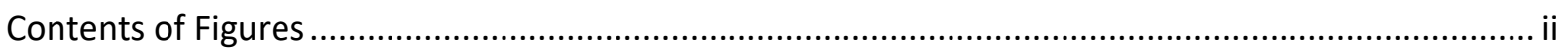

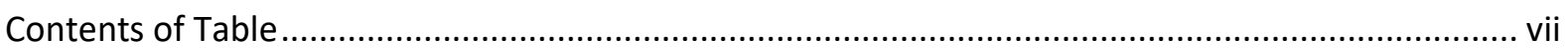

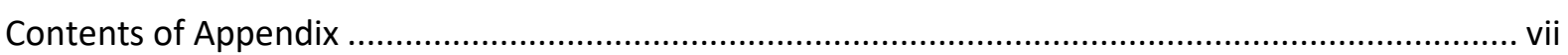

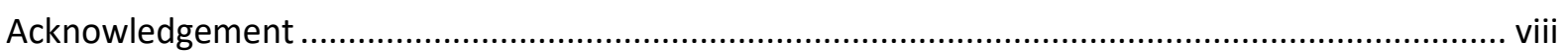

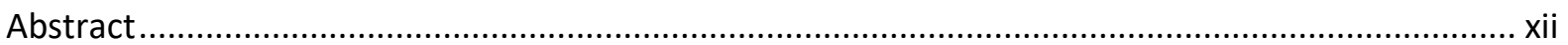

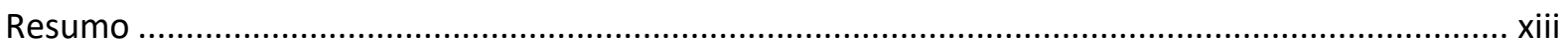

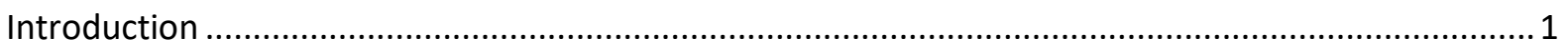

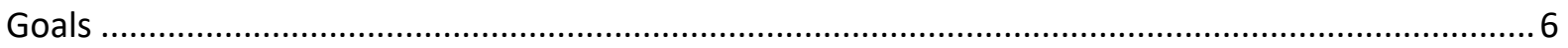

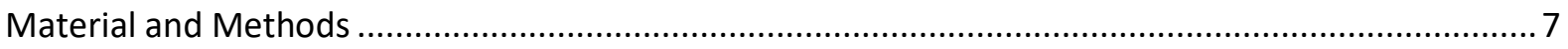

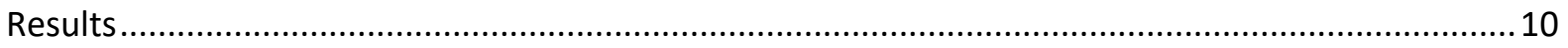

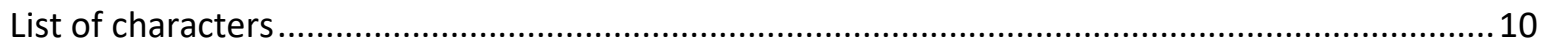

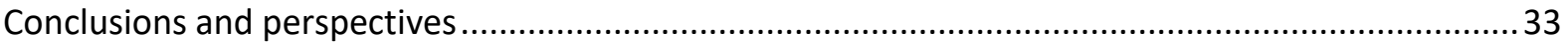

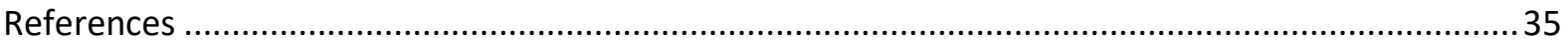

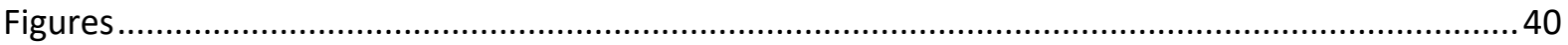

Table

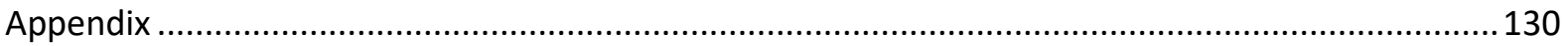




\section{Contents of Figures}

Figure 1. The single maximum parsimonious tree in phylogeny, under implied weighting scheme, setk script

Figure 2. The single maximum parsimonious tree in phylogeny, under implied weighting scheme, $k=$ 3

Figure 3. The phylogenetical relationship among the oscinellines (under implied weighting scheme, $k$ $=3$ ), with their tribal classifications in the previous literatures, front of each genus

Figures 4-8. The single maximum parsimonious tree in phylogeny, under implied weighting scheme, $k=3$, with unambiguous mapped on branches $44-48$

Figure 9a-c. Character 1, connection of head capsule with thorax .49

Figure 10a-c. Character 2, frons in dorsal view 49

Figure 11a-b. Character 3, occiput .50

Figure 12a-b. Character 4, occiput with pair of setae, basally (supercervial setae) .50

Figure 13a-c. Character 5, head in lateral view .51

Figure 14a-c. Character 6, ocellar triangle .51

Figure 15a-c. Character 7, texture of ocellar triangle .52

Figure 16a-f. Character 8 , pruinosity of ocellar triangle without tubercle .52

Figure 17a-c. Character 9, anterior end of ocellar triangle .53

Figure 18a-c. Character 10, ocellar triangle width at posterior margin .53

Figure 19a-c. Character 11, lateral margins of ocellar triangle ..............................................................54

Figure 20a-I. Character 12, color of ocellar triangle .........................................................................55

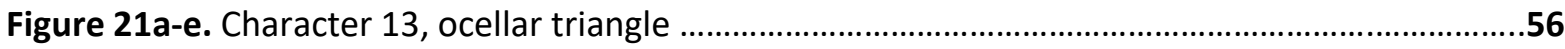

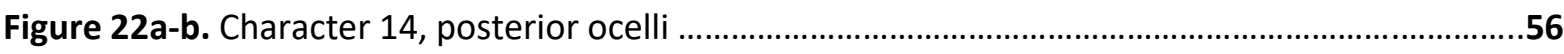

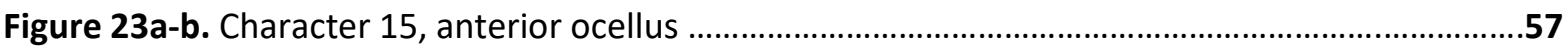

Figure 24a-c. Character 16, density of setation on frons ..................................................................57

Figure 25a-d. Character 17, anterior margin of frons in dorsal view (in comparison of the level of eyes)

Figure 26a-c. Character 18, lateral margins of frons in dorsal view ....................................................58

Figure 27a-h. Character 19, ground color of frons ..............................................................................59

Figure 28a-b. Character 20, length of orbital setae .............................................................................60

Figure 29a-b. Character 21, comparison of length of orbital setae to each other ................................60

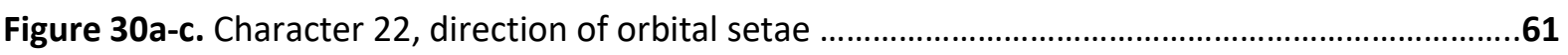

Figure 31a-c. Character 23, number of orbital setae ..........................................................................61 
Figure 32a-b. Character 24, inner vertical seta

Figure 33a-b. Character 25, length of ocellar setae

Figure 34a-f. Character 26, direction and orientation of ocellar setae

Figure 35a-b. Character 27, length of post-ocellar setae ....................................................................63

Figure 36a-c. Character 28, orientation of postocellar setae ...............................................................64

Figure 37a-b. Character 29, anterior interfrontal setae ......................................................................64

Figure 38a-c. Character 30, interfrontal setae relative to the frontal setae .........................................65

Figure 39a-d. Character 31, direction of interfrontal seta .................................................................65

Figure 40a-c. Character 32, height of gena relative to the height of first flagellomere of antenna ....66

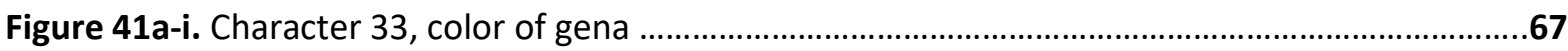

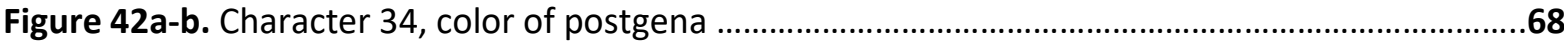

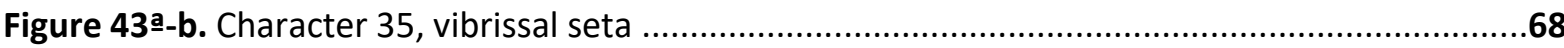

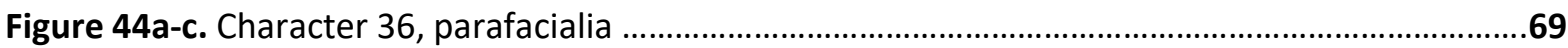

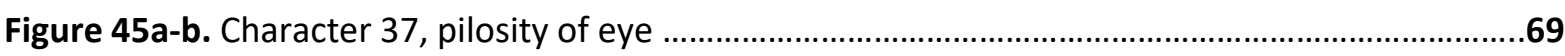

Figure 46a-b. Character 38, dispersal pilosity of eye ...............................................................................70

Figure 47a-b. Character 39, posterior margin of eye, $1 / 4$ ventral .............................................................70

Figure 48: Character 40, rate of eye compared with head capsule vertically in lateral view ...............71

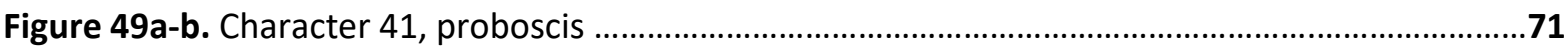

Figure 50a-b. Character 42, length of palpus in comparison with the oral cavity (lower margin of the

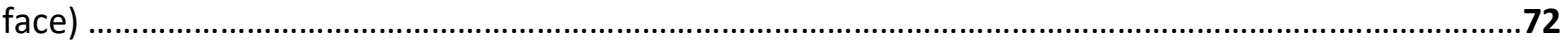

Figure 51a-b. Character 43, shape of facial carina ...........................................................................72

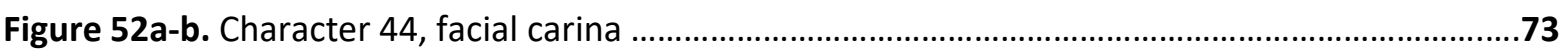

Figure 53: Character 45, rate of first antennal flagellomere (length/wide) ...........................................73

Figure 54a-d. Character 46, first antennal flagellomere distally ................................................................74

Figure 55a-g. Character 47, color of first antennal flagellomere …......................................................75

Figure 56a-b. Character 48, posterior end of first antennal flagellomere, in frontal view ...................76

Figure 57a-b. Character 49, color of basal segment of arista .................................................................76

Figure 58a-b. Character 50, color of distal segment of arista .................................................................77

Figure 59a-b. Character 51, width of distal half arista ......................................................................77

Figure 60 a-b. Character 52, position of arista on first antennal flagellomere ......................................78

Figure 61 a-b. Character 53, pilosity of entire arista comparing with the stem of arista ......................78

Figure 62a-b. Character 54, anterior part of mesonotum ..................................................................79

Figure 63: Character 55, length of scutum dorsally (width/length) .....................................................79 
Figure 64a-b. Character 56, acrostical seta

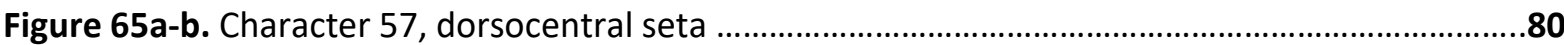

Figure 66a-b. Character 58, pruinosity all over the scutum ...............................................................81

Figure 67a-b. Character 59, color of pruinosity on scutum ...............................................................81

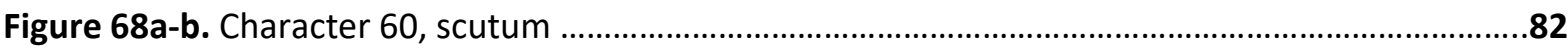

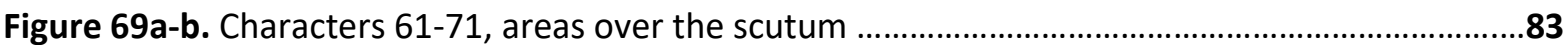

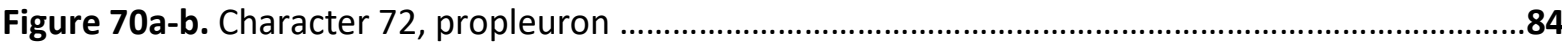

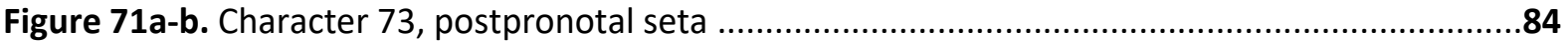

Figure 72a-b. Character 74, postpronotal seta orientation, in case of 2 or more setae ......................85

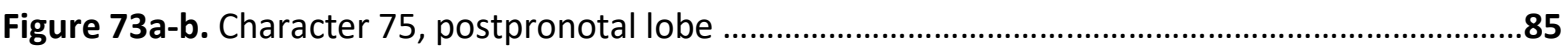

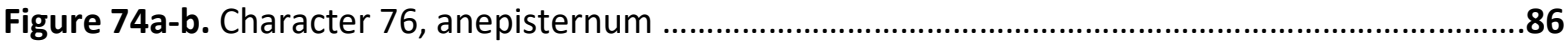

Figure 75a-b. Character 77, anepisternum setaceous ..............................................................................86

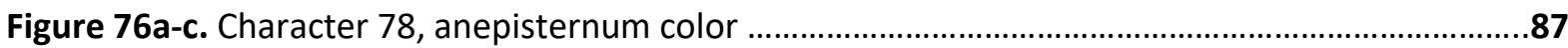

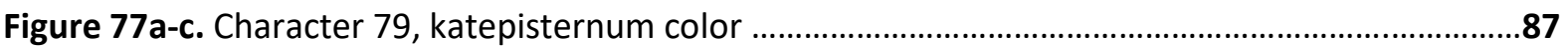

Figure 78a-c. Character 80, meron color ………...............................................................................88

Figure 79a-d. Character 81, number of distinct anterior notopleural setae ..........................................88

Figure 80a-c. Character 82, number of distinct posterior notopleural setae ......................................89

Figure 81a-b. Character 83, purinosity on postpronotal lobe ...............................................................89

Figure 82a-c. Character 84, numbers of supraalars …….......................................................................90

Figure 83a-c. Character 85, numbers of postalars ........................................................................90

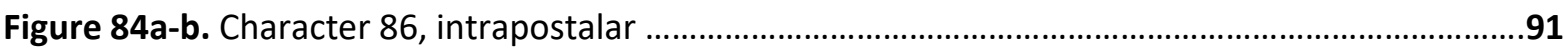

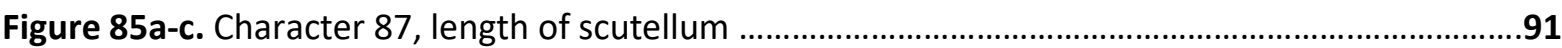

Figure 86a-b. Character 88, tip of scutellum in dorsal view ..................................................................92

Figure 87a-b. Character 89, laterals of scutellum in dorsal view ........................................................92

Figure 88a-c. Character 90, scutellar finger-like tubercles …..................................................................93

Figure 89a-b. Character 91, scutellar large setae position ......................................................................93

Figure 90a-d. Character 92, scutellum color ......................................................................................94

Figure 91: Character 93, wing length compared to abdomen length ..................................................95

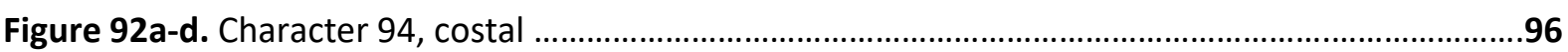

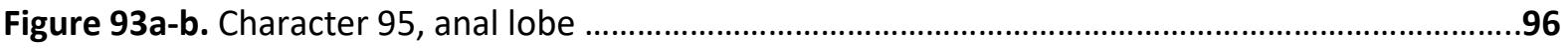

Figure 94: Character 96, alula (length/width) …………................................................................97

Figure 95: Characters 97-98; C97, pattern on the wing, apically; C98, pattern on the wing, basally; C99, pattern on the wing, medially 
Figure 96: Character 100, shape of pattern on the wing .98

Figure 97 a-b. Character 101, basal radial cell relating to the basal medial cell .98

Figure 98: Character 102, height of basal radial cell .99

Figure 99a-b. Character 103, sinusoidal of CuA1 (flexure part) .99

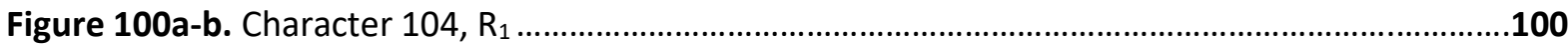

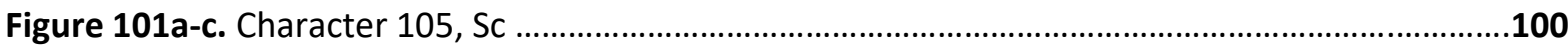

Figure 102a-b. Character 106, costal break on Sc part .........................................................................100

Figure 103: Character 107, costal break on humeral part of wing ..................................................101

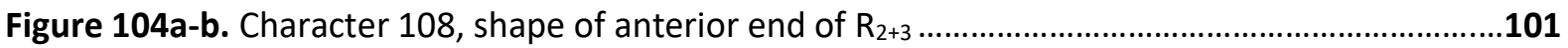

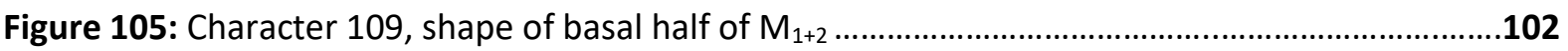

Figure 106: Character 110, shape of dm-cu ......................................................................................102

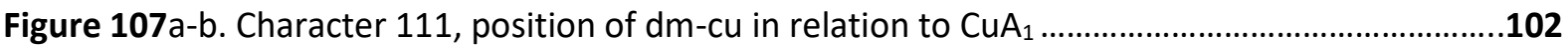

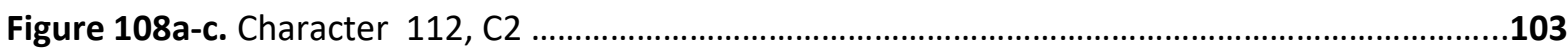

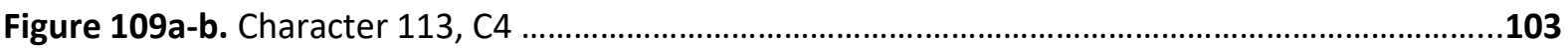

Figure 110a-b. Character 114, $\mathrm{M}_{1+2}$ thickness .................................................................................103

Figure 111a-b. Character 115-116; C115, halter color (knob); C116, halter color (stem) ..................104

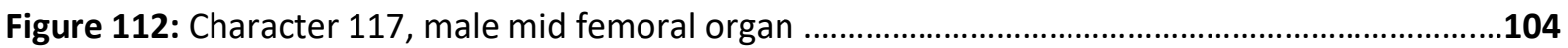

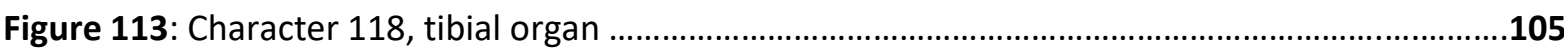

Figure 114a-b. Character 119, hind tibial spur ……….......................................................................105

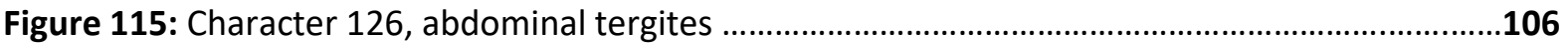

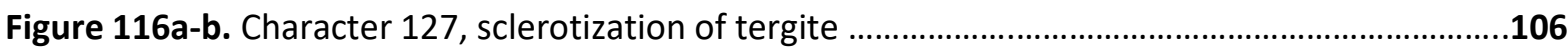

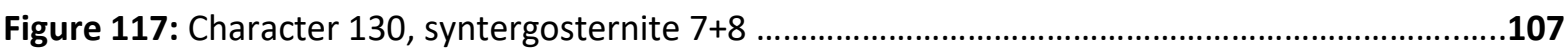

Figure 118: Character 131, epandrium medial line (dorsal sulcus) ......................................................107

Figure 119a-b. Character 132, antero-ventral corner of epandrium in posterior view .......................108

Figure 120a-b. Character 133, size of surstylus (comparing with the size of epandrium) ..................108

Figure 121a-b. Character 134, elongated setae at the edge of anterior lateral margins of surstylus109

Figure 122: Character 135, elongated setae at lateral margins of surstylus modified to spine .........109

Figure 123a-b. Character 136, varginal of surstylus in posterior view ............................................110

Figure 124a-d. Character 137, anterior and posterior lateral margins of surstylus ............................111

Figure 125a-b. Character 138, location of cercus on epandirum ......................................................112

Figure 126a-b. Character 139, cercus articulation .........................................................................112

Figure 127a-e. Character 140, length of cercus ...................................................................................113 
Figure 128a-c. Character 141, elongated setae at tip of cercus

Figure 129a-d. Character 142, cerci

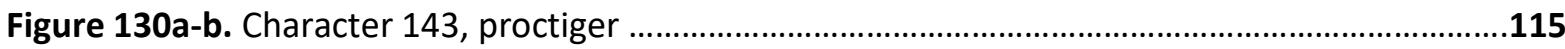

Figure 131a-c. Character 144, proctiger in posterior view ........................................................115

Figure 132a-c. Characters 145-146; C145, shape of proctiger in lateral margins (posterior view);

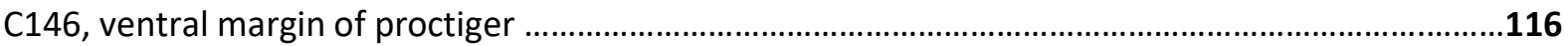

Figure 133: Character 146, ventral margin of proctiger ................................................................116

Figure 134a-b. Character 147, sub-anal plate (under proctiger) ..................................................117

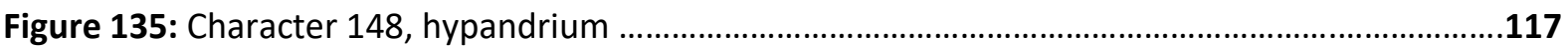

Figure 136a-b. Character 149-150; C149, shape of hypandrium; C150, phallapodeme length .........118

Figure 137: Character 150-151; C150, phallapodeme length and shape of base of phallapodeme;

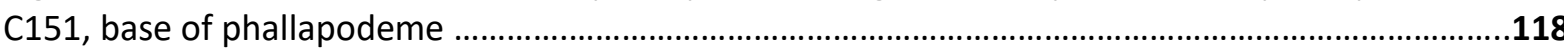

Figure 138: Character 151, base of phallapodeme ...................................................................119

Figure 139a-d. Character 152, phallapodem sclerite ..................................................................120

Figure 140a-b. Character 153, length of endophalic process ........................................................121

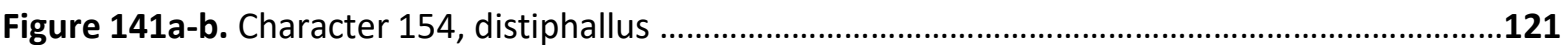

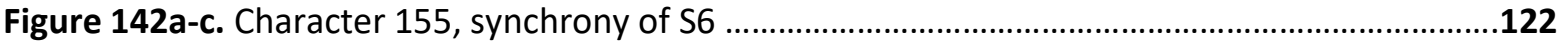

Figure 143a-b. Character 156, hypandrium, anterior view ..........................................................122

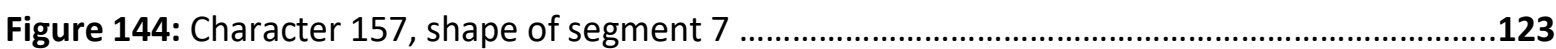

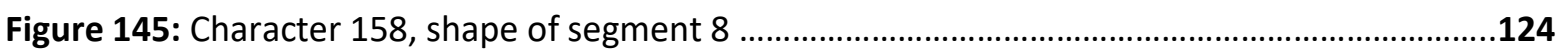

Figure 146a-b. Characters 159-160; C159, sternite 8; C160, shape of sternite 8 ..........................124

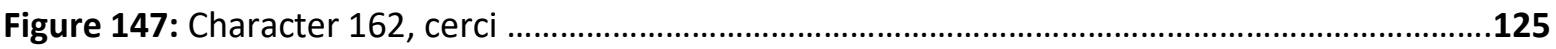

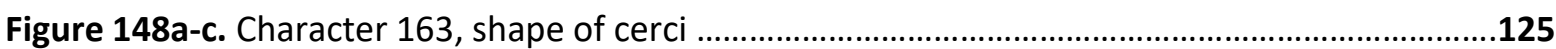

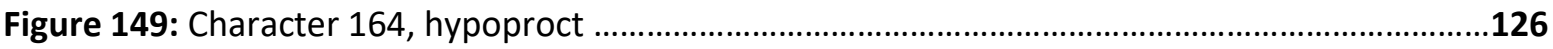

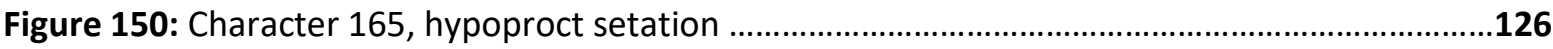

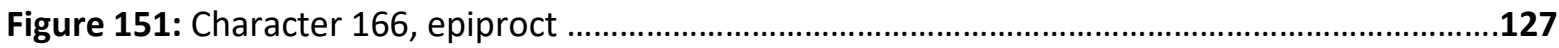

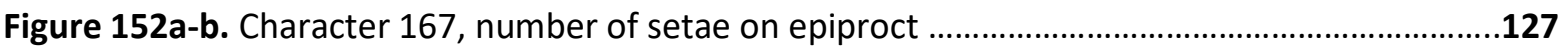




\section{Contents of Table}

Table 1. Morphological data matrix

\section{Contents of Appendix}

Appendix I. Placement of the genera of Oscinellinae and Rhodesiellinae into tribes or genus groups in different papers .131

Appendix II. List and distribution of the genera of Oscinellinae of the world 137

Appendix III. List of species examined in this study, with label details 140 


\section{Acknowledgement}

Before I say anything, I would love to thank God for everything, for all of the opportunities and kindness that were given to me, always thankful for God's endless love.

During the process of this study, there are so many people who kindly taught, helped and supported me in anytime and anywhere and I am really always so grateful to all of them.

I am extremely grateful to my advisor, Dalton de Souza Amorim, who with his exceptional kindness, support, guidance and patience taught and helped me. I am so thankful for the opportunity that he gave me and teaching me everything with passion. Also I am enormously grateful to him for the revision of this thesis with patience. It is/was always my honor to be his student.

I am also very grateful to my previous advisor, Vera Cristina Silva, who taught, helped and supported me so kindly during the first two years of my PhD study, before her retirement. I appreciate all of her kindness and help forever.

I would like to thank so much to the Coordenação de Aperfeicoamento de Pessoal de Nível Superior (CAPES), which provided me the financial support during my four years doctoral study.

So grateful and thankful to Faculdade de Filosofia, Ciências e Letras de Ribeirão Preto (FFCLRP), the organizers, evaluators, technicians, staff, secretaries and cleaners and anyone else, without whom, getting through this process would not have been possible.

I am extremely thankful to Maria Isabel, the technician of the laboratory, who always kindly helped and taught me everything, and would always be there with me at any time. Thankful to you, forever, and I really love you!

I am so much grateful and thankful to the collaborations and friendships of my friends in the Diptera Morphology and Evolution Lab, Depto. de Biologia, FFCLRP, Universidade de São Paulo and other Universities in Brazil, Diego Fachin, Gabriela Pirani, Heloisa Flores, Livia Frare, Paula Riccardi, Caleb Martins, Willian Teixeira, Alan Barbosa, Danilo Ament, Rafaela Falaschi, Sarah Oliveira, Renato Capellari, Marco Marinho and Marco Gottschalk. Without your friendship and help it was impossible to me to pass through all these.

I am forever thankful to the friendship and cooperation of my friends in other laboratories and Universities, Amanda Prato, Luana Santos, Jeferson Pedrosa, Lohan Valadares, Ruth Pele, Savio Costa, Eduardo Soares, Fabio Oliveira, Fabiano Stefanello, Stephania Sandoval, Will Franca, Diego Assis, Jaqueline Batista, Felipe Freitas, Isamara Mendes, Wafa AlHalabi, Priscila Oliveira, Neto Marcel, Ana Motta, Nanda Lima, Levi Barros, Kat Barrios, Daercio Lucena, Diego Sasso. Your presence and friendship to me means everything. Always thankful for that. 
Thank you so much to my great friend Rafael Carvalho, who supported me in any way he could, in any situation. Thank you so much for your great friendship, thank you for everything really.

My special thanks to my lovely sister, Thamiris Sipriano, and to her parents Margareth and Josemar Sipriano. You were/are always with me in any situation, supported me and kindly welcomed me to your open hearts and arms. I love you so much really.

Another special thank to my other lovely sister, Luana Santos, who always stayed with me and supported me with her warm heart and help. I love you so much really.

To my great friend and sister, Carmen Mima, who with her special lovely heart welcomed and helped me when I got to Brazil, and taught me the Portuguese language, without any expectation. I love you so much.

My respect and very special thanks and love to my great friends, Gail Kampmeier and Roger Thomason, who all the time, during many years, always supported and helped me, in any way they could, especially in English language. God bless you always.

To my great friend Livia and her lovely family, who always helped and supported me with so much kindness. I will never ever forget their warm hearts and kind help. I love you so much.

My enormous thanks to the family of Artoni. For everything I am thankful, any help, kindness, support, and their love. Always your heart and arms were/are open to me, and always welcomed me in any time and any situation. I love you so much really.

There are many many more friends who helped me in any way and any time I needed. I am just trying to mention their name here. Though their presence and friendship to me is everything and I always love them with the whole of my heart: Bahar Jahromi, Abu Fazel Dousti, Abdorassoul Zakerin, Behnam Behrooznam, Majid Fallahzadeh, Farzaneh Kazerani, Elena Moradi, Tais Sasahara, Fabricio Singaretti, Cynthia Prado, Maryam Halat, Marcia Machado, Camila Cardoso, Thiago Polizei, Keila Bariotto, Katia Amorim, Marilda Ribeiro, Bia Amorim, Amanda Fernandes, Joyce Hellyen, Natalia Vieira, Thays Quadros, Lizandra Amoroso, Natalia Santos, Natalia Salles, Dagmara Ramalho, Ana Nievas, Alan Rocha, Barbara Junqueira, Yagoub Ibrahim, Lucia Ferreros, Edna Celia, Flavio da Silva, Maria Elaine da Silva and many more friends who maybe I missed their name here and who even passed through my life shortly but gave me their passionate friendships.

My extreme gratitude to the great specialists, Michael von Tschirnhaus (Bielefeld University, Germany), John Ismay (Oxford University Museum of Natural History, UK), Barbara Ismay (Oxford University Museum of Natural History, UK), Emilia Nartshuk (Saint Petersburg Museum, Russia), Paula Riccardi (University of Rio de Janeiro, Brazil) for their participation in the survey who supported my work in this way and helped me with their great knowledge. Also thankful so much to 
Julia Mlynarek (McGill University, Canada) and Terry Wheeler (McGill University, Canada) (RIP), for all of their kind help.

So grateful to the curators and those involved, who with their scientific collections, for the loans and also the equipments for taking photo of the material, Australian Museum (NSW), British Museum of Natural History, London, England (NHM), Diptera Morphology and Evolution Lab, Depto. de Biologia, FFCLRP, Universidade de São Paulo (DMEL), Museu de Zoologia da Universidade de São Paulo, São Paulo, Brazil (MZUSP), Smithsonian Institution National Museum of Natural History, Washington DC, United States (USNM), the personal collection of Dr. John Waland Ismay, the Dr. Barbara Ismay for her kind helps in taking photo from the material, Museum of für Naturkunde an der Humbolt Universität, Berlin, Germany (ZMHU), California State Collection of Arthropods, California Department of Food and Agriculture, Sacramento, USA (CDFA), Naturhistorisches Museum Wien, Viena, Austria (NHMW), Queensland Museum (QM), Canadian National Collection of Insects, Arachnids and Nematodes (CNC), Michael von Tschirnhaus private collection, Germany (MVTPC) and Jari Flinck private collection, Finland (JFPC ). In addition, thank you so much to the Oxford University Museum of Natural History, Oxford, England (OUMNH), for giving the equipments to Dr. Barbara Ismay for taking photo of the material.

Now I would love to talk with my family with my own language:

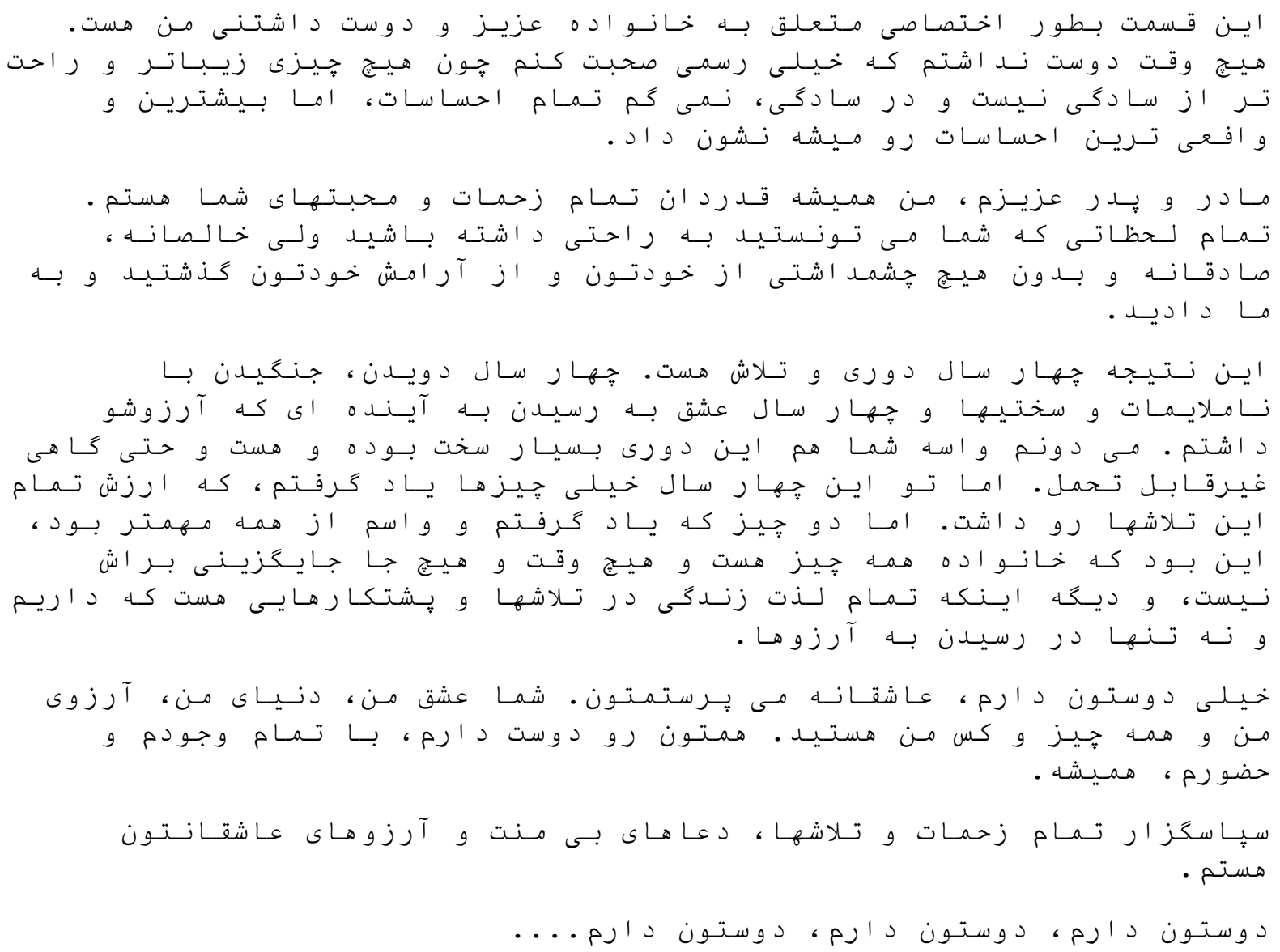


TO MY LOVING PARENTS AND LOVES OF MY LIFE. JUST LOVE YOU!

ex 


\section{Abstract}

The Chloropidae have about 3,000 described species and are often known as "grass flies": most members of the family are phytophagous and can be important pests of cereals and cultivated forage grasses. The Chloropidae have been often divided into four subfamilies Siphonellopsinae, Chloropinae, Rhodesiellinae, and Oscinellinae. The oscinellines include 124 genera in the world. This project intends to provide a systematic comparasion of the morphology of the oscinelline genera to test of the monophyly of the subfamily and the monophyly of the Rhodesiellinae, and begin to build a phylogenetic framework for the group. A total of 55 species of 50 genera of oscinellines were used as terminals, with five species of three genera of rhodesiellines, 12 species of 11 genera of chloropines, three species of two genera of siphonellopsines, two species of milichiids and one species of carnid used as outgroups. A total of 167 characters from external adult morphology (head, thorax, wing, legs, and abdomen) and from male and female genitalia have been coded and used to build a data matrix. A phylogenetic analysis was performed to reconstruct the relationships among the taxa using two different analytical parameters-implied weight with $\mathrm{k}=3$ and a $\mathrm{k}$ value $(\mathrm{k}=\sim 17)$. Both analyses resulted in one most parsimonius tree, with important differences between them on the position of many genera. The Rhodesiellinae is shown to be paraphyletic in relation to the Oscinellinae, with the Stenoscinini, a rhodesielline tribe, being sister to all remaining Oscinellinae. A large stable clade includes most Fiebriegellini and genera previously included in the Siphonellini, Tricimbini, Oscinisomatini, Liparaini, and Botanobiini. It was corroborated that the Neotropical group blanda of Oscinella does not fit together with the type-species of Oscinella and needs a group of generic rank. A large group of genera belonging to different tribes have no reliable position in the tree, suggesting that the Incertellini, Hippelatini, Oscinellini, and Siphonellini may not correspond to clades. Another large, stable clade (the "higher oscinellines") bring together the Dicraeini, and genera of the Botanobiini, Elachipterini, Liparaini, and Hippelatini. Information in the literature gives support to the idea of a clade that gathers these tribes. The use of a considerably large list of characters to analyse a larger generic taxon sampling of the subfamily will certainly help bringing stability to the classification of the group.

Key words: Acalyptratae, Chloropidae, Diptera, Phylogeny, Oscinellinae, Worldwide. 


\section{Resumo}

A família Chloropidae, têm cerca de 3.000 espécies descritas e são muitas vezes conhecidos como "moscas da grama": a maioria dos membros da família são fitófagos e podem ser importantes pragas de cereais e cultivos de gramíneas forrageiras. Essa família em sido freqüentemente dividida em quatro subfamílias: Siphonellopsinae, Chloropinae, Rhodesiellinae e Oscinellinae. Os Oscinellines incluem 124 gêneros no mundo. Este projeto pretende fornecer uma comparação sistemática da morfologia e monofilia do gênero de Oscinelinae e de Rhodesiellinae, bem como construir uma estrutura filogenética para o grupo. Um total de 55 espécies de 50 gêneros de oscinellines foram utilizados como taxon terminais, sendo cinco espécies de três gêneros de Rhodesiellines, doze espécies de onze gêneros de Chloropines, três espécies de dois gêneros de Siphonellopsines, duas espécies de Milichiideos e uma espécie de carnidos usada como grupo externo. Um total de 167 caracteres da morfologia externa de adultos (cabeça, tórax, asa, pernas e abdômen) e das genitálias masculina e feminina foram codificadas e usadas para construir uma matriz de dados. Uma análise filogenética foi realizada para reconstruir as relações entre os táxons usando dois parâmetros analíticos diferentes - peso implícito sendo $k=3$ e um valor $k(k=\sim 17)$. Ambas as análises resultaram em uma árvore mais parsimoniosa, com diferenças importantes entre si e em muitos gêneros. Rhodesiellinae mostrou-se parafilético em relação a Oscinellinae, e Stenoscinini, uma tribo de rhodesielline, irmã de todos os restantes de Oscinellinae. Um grande clado estável inclui a maioria dos gêneros Fiebriegellini anteriormente incluídos nos Siphonellini, Tricimbini,

Oscinisomatini, Liparaini e Botanobiini. Isso reafirmou que o grupo neotropical blanda de Oscinella não se encaixa com as espécies-tipo de Oscinella necessitando de uma classificação genérica para grupo. Um numeroso grupo de gêneros pertencentes a diferentes tribos não possuem uma posição confiável na árvore, sugerindo que os Incertellini, Hippelatini, Oscinellini e Siphonellini podem não corresponder aos respectivos clados. Outro amplo e estável clado (os "oscinelines superiores") reúne os Dicraeini e os gêneros de Botanobiini, Elachipterini, Liparaini e Hippelatini. Informações na literatura dão suporte à idéia de um clado que reúna essas tribos. O uso de uma lista de caracteres consideravelmente ampla que permita analisar uma amostragem genérica maior de taxons da subfamília, certamente, auxiliará e trará mais estabilidade à classificação do grupo.

Palavras-chave: Acalyptratae, Chloropidae, Diptera, Filogenia, Oscinellinae, Universal. 


\section{Introduction}

The Chloropidae correspond to a considerably large family of acalyptrate flies, with about 3,000 described species (Nartshuk 2014). Some authors estimate between 5,000 and 10,000 the real number of chloropid species worldwide (Ismay 2014). They are found in all regions of the world except Antarctica.

In temperate regions, many species are associated with grassland habitats (Ismay \& Nartshuk 2000). They are minute to small in size (1.0 to $4.0 \mathrm{~mm}$ ), rarely larger (6.0 to 9.0 $\mathrm{mm})$. They are mostly blackish, but some species may be greyish, largely yellow, or greenish. In many genera, the mesonotum often has a pattern with three to five dark longitudinal stripes against a lighter background (Nartshuk et al. 1988, Ismay 2014).

The family is often referred to as "grass flies" and has members with highly specialized biologies. Most members of the family are phytophagous and some species are important pests of cereals and cultivated forage grasses (Nartshuk 2006). Different genera have species that infest the shoots of cereal crops and grasses. Among these species, Chlorops Meigen, Elachiptera Macquart and Oscinella Becker are best known for the damage their larvae cause (Deeming \& Al-Dhafer 2012). The most important economic damages are of the Poaceae stem miners. They can live in different parts of the plant, deforming them in various ways, even producing galls (Ismay \& Nartshuk 2000). Alternatively, some species are saprophagous and live in decaying plant tissues (Ismay \& Nartshuk 2000) or in fungi (Deeming \& Al-Dhafer 2012). There are as well parasites and parasitoids of other insects and arthropods. Larvae of species of Thaumatomyia Zencker prey upon root-aphids, larvae of Batrachomyia Krefft are subdermal parasites of frogs, and larvae of Speccafrons Sabrosky develop in spider egg capsules (Deeming \& Al- Dhafer 2012).

A few genera of the family are considered vectors of diseases between animals and humans. The so-called "eye gnats" (Hippelates spp. and Liohippelates spp.) constitute important vectors, swarming around eyes and body secretions. In Brazil they may transmit purpuric fever, which initial symptom is acute conjunctivitis (Reilly et al. 2007, Deeming \& Al-Dhafer 2012).

McAlpine's (1989) study of the phylogeny of the Acalyptratae included Chloropidae as a monophyletic group. In his system, the Chloropidae fit into the Carnoidea, in which they 
are more closely related to the families Cryptochetidae and Milichiidae, what is supported by synapomorphies of the head capsule, proboscis, wing venation and spermathecae (Buck 2006). Chloropidae and Milichiidae are well-supported as sister groups due to the presence of a well developed ventral receptacle and a bare, truncate distiphallus in the male terminalia (Buck 2006).

The relationships within the Chloropidae remain in a large extent unsettled. Besides the considerably large number of species and taxa of generic rank in the family in a worldwide scale (Nartshuk \& Andersson 2013), some of the genera in the family are probably paraphyletic, thus tending to obscure the real affinities between the groups (Sabrosky 1951).

Great advance in the systematics of the Chloropidae was obtained from experts such as Andersson (1977), Kanmiya (1983), Sabrosky (1984), Nartshuk (1983), Ismay (1993), and Nartshuk \& Andersson (2013), providing the basis for the current tribal and subfamily classification of the family (Mlynarek \& Wheeler 2018). Andersson $(1977,1979)$ and Nartshuk $(1983,1987)$ presented some ideas on the phylogeny of the Chloropidae, but stressed that much further work was needed. Andersson $(1977,1979)$ remarked that the Chloropidae evolved relatively recently and, combined with the size of the chloropids, a morphology-based cladistic analysis would render difficult.

Becker (1910) divided the family Chloropidae in two main groups, now referred to the Oscinellinae and Chloropinae. Duda (1930a) divided the family into three subfamilies, Oscinellinae, Chloropinae and Paleoscinellinae (including Hippelates and related genera) (Sabrosky 1941). Later Duda (1932) changed his classification of the group to include two subfamilies, Siphonellopsinae and Chloropinae, dividing the latter into three tribes Chloropoidea, Oscinelloidea and Hippelatoidea (Sabrosky 1941). Sabrosky (1941) kept four chloropid subfamilies, Chloropinae, Oscinellinae, Siphonellopsinae and Heringiinae.

In a modern stage of the systematics of the family, Andersson (1977) recognized three subfamilies, Siphonellopsinae, Chloropinae and Oscinellinae, a position followed by Ismay \& Nartshuk (2000) and Mlynarek \& Wheeler $(2009,2018)$. Nartshuk \& Andersson (2013), in the phylogenetic section of their study, proposed the Siphonellopsinae to be sister to the set of remaining members of chloropids.

There are some synapomorphies that sustain that the Siphonellopsinae should be sister to the remaining chloropids. This subfamily often has more extensive bristling on the 
thorax, and the arrangement and orientation of cephalic setae are more similar (in terms of a plesiomorphic condition) to related families such as Milichiidae (Nartshuk \& Andersson 2013). The male genitalia is asymmetrical, which is assumed by Brake (2000) to be relatively plesiomorphic to the other chloropids. Only four genera are included by Nartshuk (2012) in this subfamily, Apotropina Hendel, Protohippellates Andersson, Siphonellopsina Andersson and Siphonellopsis Strobl. Mlynarek \& Wheeler (2018) discussed the morphological variation and the generic limits in this subfamily.

The Chloropinae contain 66 genera distributed and it has been unanimously accepted as monophyletic. The relationships within the Chloropinae have been addressed informally by Andersson (1977), Nartshuk (1983, 2012), Nartshuk \& Andersson (2013), and formally by Paganelli (2002), Riccardi (2016) and Riccardi \& Amorim (submitted). In Riccardi \& Amorim (submitted), the monophyly of the Chloropinae was corroborated and a group of stable clades has been ranked as tribes.

Nartshuk \& Andersson (2013) gave subfamily rank to the Rhodesiellinae, previously proposed as a tribe of Oscinellinae by Nartshuk (1983). Nartshuk \& Andersson (2013), in the phylogenetic section of their study, presented two alternative scenarios for the relationships in the chloropids except the Siphonellopsinae: (Chloropinae + (Rhodesiellinae + Oscinellinae)) or (Oscinellinae + (Rhodesiellinae + Chloropinae)). The number of features and the taxon sampling in their study is small and the monophyly of the Oscinellinae still needed further evidence. In other words, it was not actually clear if the "Rhodesiellini" would fit into the Oscinellinae or if it should be a clade sister to one of these two subfamilies. Nartshuk (2012) recognized three groups of genera within the Rhodesiellinae to which was given tribe rank.

The Oscinellinae now include 124 genera. For a long time, this group was referred to as Oscininae (Sabrosky 1941). This name of the group of the family was replaced by Oscinellinae, since Oscinis Laterille has been synonimyzed to Chlorops (Sabrosky 1941). Sabrosky (1980a) recorded about 245 described species for the Oscinellinae. Most species are 1 to $5 \mathrm{~mm}$ long, rather blackish, uniformly colored, with the costal vein extending to $M_{1+2}$ and usually with the presence of a male femoral organ. There is a wide variation in the subfamily, including size and color (Andersson 1977, Mlynarek \& Wheeler 2018).

Andersson (1977), as mentioned above, divided the Oscinellinae into two tribes, Oscinellini and Rhodesiellini. Andersson (1977) concentrated his taxon sampling mostly on 
the Old World fauna and did not examine many of the New World genera of the subfamily. The tribes Rhodesiellini and the Oscinellini were subdivided by Andersson (1977) into nine genus-groups: Javanoscinis, Elachiptera, Gaurax, Dicraeus, Lipara, Polyodaspis, Aphanotrigonum, Oscinella and Eribolus groups. Sabrosky (1984) dealt with most of Andersson's genus-groups as taxa without tribal rank.

Ismay (1993) addressed the question of the tribes Tricimbini and Incertellini, including a phylogenetic discussion of the relationships between groups of species of Tricimba and of Aprometopis, and of shared features between Tricimba and related genera. More recently, Nartshuk (2012) divided the subfamily Oscinellinae in 11 tribes, but the affiliation of many genera of the subfamily into the tribes were not established. The tribes are:

(1) Tribe Botanobiini Malloch, 1913: Cestoplectus Lamb, Gampsocera Schiner, Gaurax Loew, Hapleginella Duda, Leucochaeta Becker, Pselaphia Becker, Pseudogaurax Malloch, Pterogaurax Duda;

(2) Tribe Dicraeini Nartshuk, 1983: Dicraeus Loew;

(3) Tribe Elachipterini Lioy, 1864: Alombus Becker, Anatrichus Loew, Ceratobarys Coquillett, Cyrtomomyia Becker, Disciphus Becker, Elachiptera Macquart, Lasiochaeta Corti, Melanochaetomyia Cherian, Sepsidoscinis Hendel, Togeciphus Nishijima;

(4) Tribe Fiebrigellini Nartshuk, 1983: Anacamptoneurum Becker, Chaetochlorops Malloch, Epimadiza Becker, Fiebrigella Duda, Lasiambia Anonymous, Heteroscinis Lamb, Heteroscinoides Cherian, Polyodaspis Duda;

(5) Tribe Hippelatini Duda, 1932: Chaethippus Duda, Hippelates Loew, Liohippelates Duda, Lioscinella Duda;

(6) Tribe Incertellini Nartshuk, 1983: Apallates Sabrosky, Aphanotrigonum Duda, Biorbitella Sabrosky, Incertella Sabrosky, Malloewia Sabrosky, Meijerella Sabrosky, Microcercis Beschovski, Parameijerella Cherian, Parapallates Cherian \& Tilak;

(7) Tribe Liparaini Camero \& Tubbs, 1990: Anomoeoceros Lamb, Calamoncosis Enderlein, Dasyopa Malloch, Lipara Meigen, Pseudeurina Meijere;

(8) Tribe Oscinellini Becker, 1910: Camptoscinella Sabrosky, Conioscinella Duda, Olcella Enderlein, Oscinella Becker, Oscinimorpha Lioy, Neolcella Cherian, Neoscinella Sabrosky; 
(9) Tribe Oscinisomatini Enderlein, 1911: Eribolus Becker, Oscinisoma Lioy, Rhopalopterum Duda, Sabroskyina Beschovski;

(10) Tribe Siphonellini Lioy, 1864: Kurumemyia Kanmiya, Siphonella Macquart, Speccafrons Sabrosky;

(11) Tribe Tricimbini Nartshuk, 1983: Aphanotrigonella Nartshuk, Aprometopis Becker, Indometopis Cherian, Siphunculina Rondani, Pseudotricimba Ismay, Tricimba Lioy, Tricimbomyia Cherian.

Mlynarek \& Wheeler (2018) for the first time made a formal phylogenetic analysis in the subfamily, with a study of 75 species of nine genera of Elachipterini, with some of the genera of oscinellines as outgroups. Two major clades were recognized, the group Anathricus and the group Elachiptera. In Appendix I, we provide a comparison of Andersson's (1977), Kanmiya's (1983), Nartshuk's (1983, 2012), and Mlynarek \& Wheeler's (2018) systems concerning the position of the Oscinellinae and Rhodesiellinae genera into genus groups or tribes.

Andersson (1977), Kanmiya (1983), Nartshuk (1983) and Mlynarek \& Wheeler (2018) worked on the question of the position and relationships of genera within the system of the Oscinellinae/Rhodesiellinae. The picture drawn out from their papers is that a global approach of the Oscinellinae has not been possible so far. Many genera have not been dealt in each of these studies, there is considerable inconsistency between their conclusions, and strict phylogenetic inference was used only by Mlynarek \& Wheeler (2018). This strongly points to the need of a consistent comparative study of the genera of Oscinellinae and of their relationships so monophyly of the subfamily (including or excluding the Rhodesiellinae) can be verified and major clades within them can be detected. 


\section{Goals}

This study intends to perform a careful comparative study of the morphology of the genera of Oscinellinae to develop the basis for a formal phylogenetic analysis of the subfamily. Hence, the main goals of the thesis are:

(1) to describe in considerable detail morphological features not addressed in the literature for many genera and as much as possible convert this information into characters;

(2) to test of the monophyly of the Oscinellinae, which basically means to check the position of the Rhodesiellinae relative to the Oscinellinae;

(3) to establish the phylogenetic relationships between the genera of oscinellines, trying to recover major clades; and

(4) within the limits of the taxon sampling, to verify the existence of paraphyletic genera in the system. 


\section{Material and Methods}

A complete list of the genera of Oscinellinae and Rhodesiellinae is in Appendix II, with indication of the geographic distribution of each genus and the origin of the typespecies. We tried to use representatives of as many genera as possible. Whenever available, we preferred to examin the type-species of the genus. The Neotropical fauna of the subfamily was the easiest source of material from the oscinelline genera, but material from other regions have been requested and included in the analysis when made available.

A list of the specimens received for this project is presented in Appendix III, indicating gender and corresponding depositary institutions.

Abbreviations of institutions from which material was obtained are indicated below:

- $\quad$ Australian Museum (NSW);

- $\quad$ British Museum of Natural History, London, England (NHM);

- Diptera Morphology and Evolution Lab, Depto. de Biologia, FFCLRP, Universidade de São Paulo (DMEL);

- Museu de Zoologia da Universidade de São Paulo, São Paulo, Brazil (MZUSP);

- Smithsonian Institution National Museum of Natural History, Washington DC, United States (USNM);

- $\quad$ Dr. John Waland Ismay personal collection (JIPC), from which Barbara Ismay very kindly made photos of some species (referred to ahead);

- Museum of für Naturkunde an der Humbolt Universität, Berlin, Germany (ZMHU);

- California State Collection of Arthropods, California Department of Food and Agriculture, Sacramento, USA (CDFA);

- $\quad$ Naturhistorisches Museum Wien, Viena, Austria (NHMW);

- Queensland Museum (QM);

- $\quad$ Canadian National Collection of Insects, Arachnids and Nematodes (CNC);

- Michael von Tschirnhaus private collection, Bielefeld University, Germany (MVTPC);

- Jari Flinck private collections, Finland (JFPC)

- Zeinab Bazyar private collection of material from Iran and from donations from countries other than Brazil (ZBPC). 
This analysis is based on the study of the morphology of more than 5.000 specimens of the Diptera Morphology and Evolution Lab and on loan of material from different museums and collections of the world. The material in our data matrix includes males and/or females of 61 species of 54 genera of Oscinellinae (including the type-species of 38 genera), six species of four genera of Rhodesiellinae (including the type-species of one genus), 14 species of 13 genera of Chloropinae, three species of two genera of Siphonellopsinae, three species of two genera of Milichiidae, and one species of Carnidae. Some species were made available only through photos sent by museum curators. Part of the data was obtained or confirmed from the literature, particularly Andersson (1977), Chvala et al. (1974), Cherian \& Emiliyamma (1999), Kanmiya $(1983,1994)$, Kubik \& Bartak (2010), Liu et al. (2017), Malloch (1913b, 1928, 1938), Mlynarek \& Wheeler (2009, 2018), Sabrosky $(1941,1947)$, Spencer (1978), Walker (1860). The entire tree was rooted on the species of Carnidae.

Some of the illustrations were made vectorizing Dr. Paula Raile Riccardi's original drawings with her consent.

The dissection of specimens followed procedures that are standard for some groups of Diptera. The male terminalia was removed from the abdomen and placed into warm $10 \%$ $\mathrm{KOH}$ for 2-3 minutes. They were then transferred to acetic acid and, afterwards, to ethanol $70 \%$ for a few seconds. After dissection, the wing, abdomen, and the terminalia were stored in a microvial containing glycerol and pinned together with the specimen. In some cases, wings were mounted between cover slips with Euparal and pinned together the specimen. The female terminalia were removed from the abdomen and placed into cold $\mathrm{KOH}$ for 5-15 hours and then transferred to acetic acid. They were then transferred to ethanol $70 \%$ for a few seconds where the ovipositor was pulled out. The abdomen was transferred to a microvial with glycerol, which was pinned under the specimen.

Photos were taken using a DC500 Leica camera coupled to a Leica MZ16 stereomicroscope. Helicon Focus Software 6 was used for stacking. Photoshop CC was used for further editing of images. Initial drawings were made using a camera lucida coupled to a Leica DM2500 microscope, vectorized with Illustrator CC.

Morphology and terminology basically follows Cumming \& Wood (2009), except for particular chloropid features, that follow Andersson (1977), Kanmiya (1983) and Nartshuk \& Andersson (2013). 
The morphological matrix was constructed with WinClada (Nixon 1999-2002). All multistate characters were treated as unordered. Characters were polarized through the rooting between outgroups (Nixon \& Carpenter, 1993), in this case, specifically on Carnidae. Matrix analysis followed the cladistic method under the principle of parsimony (Lipscomb 1998, Schuh \& Brower 2009), carried out with the software TNT ver. 1.1 (Goloboff et al. 2008b). Both equal and implied weighting schemes (Goloboff 1993, Goloboff et al. 2005) were conducted with the "New Technology Search" option (random seed 0, irritation level 75, initial addseqs 11, finding minimum tree length 10 times, default values for Ratchet, Drift, Sectorial search and Tree fusion) saving the most parsimonious trees (MPTs). There is a major loss of information using equal weight and the trees obtained therefore were not used. The tree from both implied weight strategies had better resolution and are able to recognize and underscore highly incongruent characters. We used two different implied weighting schemes, one with $\mathrm{k}=3$, and the other with $\mathrm{k}=\sim 17$ using the setk.run script. Both trees represented in the results (Figures 1, 2). 


\section{Results}

The complete data matrix has 167 morphological characters for 84 terminal taxa. Along the stage of the analysis of the matrix, six terminals had a large amount of missing data-Cadrema lonchopteroides (NHM), Camptoscinella annulitibia (NHM), Deltastoma unipunctatum (NSW), Eugaurax floridensis (USNM), Meijerella cavernae (ZMHU) and Pterogaurax sp. nov. (DMEL)-and were removed from the final analyses. Part of the characters come from published morphological studies-as Andersson (1977), Kanmiya (1983), Nartshuk (1983), Nartshuk (2012) —or from phylogenetic analyses - Mlynarek \& Wheeler (2018) and Riccardi \& Amorim (submitted) -, sometimes with slightly different coding and the sources are duely recognized.

\section{List of characters}

\section{Head}

1. Connection of head capsule with thorax (Figure 9a-c): (0) ventrally; (1) posteriorly; (2) medially.

2. Frons (in dorsal view) (Figure 10a-c): (0) wider than long; (1) as wide as long; (2) longer than wide. Riccardi \& Amorim (submitted), Mlynarek \& Wheeler (2018)

3. Occiput: (0) convex (Figure 11a-b): (1) straight, angulated with frons. Riccardi \& Amorim (submitted), Mlynarek \& Wheeler 2018

4. Occiput with pair of setae, basally (supercervial setae) (Figure 12a-b): (0) absent; (1) present.

5. Head in lateral view (Figure 13a-c): (0) longer than wide; (1) as wide as long; (2) wider than long. Mlynarek \& Wheeler 2018

6. Ocellar triangle (Figure 14a-c): (0) well marked, margins of ocellar triangle conspicuous; (1) not well marked, margins not clear or entirely absent. Riccardi \& Amorim (submitted)

7. Texture of ocellar triangle (Figure 15a-c): (0) opaque, rugose; (1) shining. Mlynarek \& Wheeler (2018)

8. Pruinosity of ocellar triangle without tubercle (Figure 16a-f): (0) almost entirely pubescent; (1) posterior two third pubescent; (2) posterior third pubescent; (3) almost 
bare; (4) anterior two third pubescent; (5) anterior third pubescent; (6) anterior tip of triangle bare; (7) anterior tip of triangle pubescent. Riccardi \& Amorim (submitted)

9. Anterior end of ocellar triangle (Figure 17a-c): (0) reaching or almost reaching anterior margin of frons; (1) between anterior margin of frons and middle of frons; (2) at most reaches middle of frons. Riccardi \& Amorim (submitted), Mlynarek \& Wheeler (2018)

10. Ocellar triangle width at posterior margin (Figure 18a-c): (0) hardly wider than the tubercle itself; (1) wider than tubercle, reaching halfway to eye margin; (2) getting close or laterally reaching the eye margins. Riccardi \& Amorim (submitted)

11. Lateral margins of ocellar triangle (Figure 19a-c): (0) convex; (1) concave; (2) straight. Mlynarek \& Wheeler (2018)

12. Color of ocellar triangle (Figure 20a-I): (0) light brown to yellow entirely; (1) dark brown to black entirely; (2) anterior end light, the rest dark; (3) anterior half light, posterior half dark; (4) posterior end light, the rest dark; (5) posterior light, anterior dark; (6) middle (oval or linear) light, around dark; (7) posterior end dark, the rest light; (8) anterior fifth and a median band dark, with dark yellow background; (9) dark tubercle with darker lateral areas over lighter background.

13. Ocellar triangle (Figure 21a-e): (0) concolor; (1) with spot; (2) with band.

14. Posterior ocelli (Figure 22a-b): (0) yellow or light brown; (1) black or dark brown.

15. Anterior ocellus (Figure 23a-b): (0) yellow or light brown; (1) black or dark brown.

16. Density of setation on frons (Figure 24a-c): (0) bare; (1) dense; (2) scattered.

17. Anterior margin of frons in dorsal view (in comparison of the level of eyes) (Figure 25a-d): (0) straight or slightly projected; (1) projected moderately beyond the anterior margin of eyes; (2) concave, medially with projection; (3) concave, medially straight. Riccardi \& Amorim (submitted)

18. Lateral margins of frons in dorsal view (Figure 26a-c): (0) converging towards anterior margin; (1) diverging towards anterior margin; (2) almost parallel. Riccardi \& Amorim (submitted), Mlynarek \& Wheeler (2018)

19. Ground color of frons (Figure 27a-h): (0) anterior third yellow; (1) anterior half yellow; (2) anterior two third yellow; (3) entirely yellow; (4) entirely dark; (5) anterior third dark; (6) anterior two third dark; (7) anterior half dark.

20. Length of orbital setae (Figure 28a-b): (0) long; (1) short. Riccardi \& Amorim (submitted) 
21. Comparison of length of orbital setae to each other (Figure 29a-b): (0) of same length; (1) some clearly longer than others. Mlynarek \& Wheeler (2018)

22. Direction of orbital setae (Figure 30a-c): (0) all reclinate; (1) all proclinate; (2) anterior one proclinate, posterior two reclinate. Riccardi \& Amorim (submitted)

23. Number of orbital setae (Figure 31a-c): (0) less than five; (1) between five and ten; (2) more than ten. Riccardi \& Amorim (submitted) In all cases the last posterior seta, which was different to the rest, was ignored.

24. Inner vertical seta (Figure 32a-b): (0) conspicuous and strong; (1) absent or not distinguishable. Mlynarek \& Wheeler (2018)

25. Length of ocellar setae (Figure 33a-b): (0) shorter than or as long as ocellar tubercle; (1) longer than ocellar tubercle. Riccardi \& Amorim (submitted), Mlynarek \& Wheeler 2018

26. Direction and orientation of ocellar setae (Figure 34a-f): (0) proclinate and convergent; (1) proclinate and divergent; (2) proclinate and parallel; (3) reclinate and convergent; (4) reclinate and parallel; (5) reclinate and divergent. Riccardi \& Amorim (submitted), Mlynarek \& Wheeler 2018

27. Length of post-ocellar setae (Figure 35a-b): (0) shorter or as long as the ocellar tubercle; (1) longer than the ocellar tubercle.

28. Orientation of postocellar setae (Figure 36a-c): (0) convergent; (1) divergent; (2) parallel.

29. Anterior interfrontal setae (Figure 37a-b): (0) longer than remaining interfrontal setae; (1) shorter or as long as remaining interfrontal setae.

30. Interfrontal setae relative to the frontal setae (Figure 38a-c): (0) absent; (1) present, as long as frontal setae; (2) present, conspicuous. Riccardi \& Amorim (submitted)

31. Direction of interfrontal seta (Figure 39a-d): (0) all proclinate; (1) all inclinate; (2) anteriorly proclinate and posteriorly inclinate; (3) all straight.

32. Height of gena relative to the height of first flagellomere of antenna (Figure 40a-c): (0) shorter; (1) almost as long; (2) longer. Riccardi \& Amorim (submitted)

33. Color of gena (Figure 41a-i): (0) all over yellow or light brown; (1) posterior half yellow; (2) anterior half yellow; (3) dark color; (4) posterior third yellow; (5) anterior third yellow; (6) posterior two third yellow; (7) anterior two third yellow; (8) all over yellow except anterior tip dark. 
34. Color of postgena (Figure 42a-b): (0) black or dark brown; (1) yellow or light brown.

35. Vibrissal seta (Figure 43a-b): (0) present; (1) absent.

36. Parafacialia (related to the broadness of first flagellomere of antenna) (Figure 44a-c): (0) absent; (1) narrow; (2) wider. Riccardi \& Amorim (submitted)

37. Pilosity of eye (Figure 45a-b): (0) bare; (1) hairy.

38. Dispersal pilosity of eye (Figure 46a-b): (0) scattered; (1) dense.

39. Posterior margin of eye, $1 / 4$ ventral (Figure $47 a-b)$ : (0) continues; (1) with notch.

40. Rate of eye compared with head capsule vertically in lateral view (Figure 48): (0) smaller than 1.3; (1) between 1.3 and 1.4; (2) between 1.41 and 1.5; (3) between 1.51 and 1.6; (4) bigger than 1.6.

41. Proboscis (Figure 49a-b): (0) geniculate; (1) not geniculate.

42. Length of palpus in comparison with the oral cavity (lower margin of the face) (Figure 50a-b): (0) at most at the margin of oral cavity; (1) longer than the margin of oral cavity. Mlynarek \& Wheeler (2018)

43. Shape of facial carina (Figure 51a-b): (0) wide dorsally and ventrally; (1) narrow ventrally and wide or narrow dorsally.

44. Facial carina (Figure 52a-b): (0) complete, reaching to the epistomal margin or very close; (1) incomplete, extending almost halfway to epistomal margin. Riccardi \& Amorim (submitted), Mlynarek \& Wheeler 2018

45. Rate of first antennal flagellomere (length/wide) (Figure 53): (0) 1; (1) between 1.1 and $1.5 ;(2)$ more than 1.5 .

46. First antennal flagellomere distally (Figure 54a-d): (0) pointed; (1) angulated; (2) rounded. Riccardi \& Amorim (submitted)

47. Color of first antennal flagellomere (Figure 55a-g): (0) posterior half yellow or light; (1) entirely yellow or light brown; (2) entirely dark brown or black; (3) posterior two third yellow or light brown; (4) entirely yellow or light brown except at base of arista; (5) yellow or light brown, dark dorsobasally; (6) posterior third yellow or light brown; (7) dark with yellow ventral margin. Riccardi \& Amorim (submitted)

48. Posterior end of first antennal flagellomere, in frontal view (Figure 56a-b): (0) straight; (1) concave (reniform). Riccardi \& Amorim (submitted), Mlynarek \& Wheeler 2018

49. Color of basal segment of arista (Figure 57a-b): (0) dark; (1) yellow. 
50. Color of distal segment of arista (Figure 58a-b): (0) dark; (1) yellow. Riccardi \& Amorim (submitted)

51. Width of distal half arista (Figure 59a-b): (0) thin; (1) thick. Riccardi \& Amorim (submitted), Mlynarek \& Wheeler 2018

52. Position of arista on first antennal flagellomere (Figure 60a-b): (0) placed at most equal with arista base; (1) more than arista base, at least twice. Riccardi \& Amorim (submitted)

53. Pilosity of entire arista comparing with the stem of arista (Figure 61a-b): (0) short hairs; (1) long hairs. Riccardi \& Amorim (submitted), Mlynarek \& Wheeler 2018

\section{Thorax}

54. Anterior part of mesonotum (Figure 62a-b): (0) not projected; (1) projected (neck form).

55. Length of scutum dorsally (Figure 63): (0) as long as wide; (1) longer than wide; (2) wider than long. Mlynarek \& Wheeler (2018)

56. Acrostical seta (Figure 64a-b): (0) present; (1) absent.

57. Dorsocentral seta (Figure 65a-b): (0) present; (1) absent. Riccardi \& Amorim (submitted)

58. Pruinosity all over the scutum (Figure 66a-b): (0) present; (1) absent. Riccardi \& Amorim (submitted)

59. Color of pruinosity on scutum (Figure 67a-b): (0) dark brown or black; (1) yellow or whitish.

60. Scutum (Figure 68a-b): (0) no longitudinal sulcus; (1) with 1-3 longitudinal sulcus. Riccardi \& Amorim (submitted)

61. Area over dc (Figure 69a): (0) dark; (1) yellow.

62. Area over ac (Figure 69a): (0) dark; (1) yellow.

63. Lateral area, dorsal to margin on scutum (Figure 69a): (0) dark; (1) yellow.

64. Lateral most area on scutum (Figure 69a): (0) dark; (1) yellow.

65. Scutum dorsal to wing base (Figure 69a): (0) dark; (1) yellow.

66. Scutum posterior end (Figure 69a): (0) dark; (1) yellow.

67. Scutum area between ac and dc (Figure 69a): (0) dark; (1) yellow. Riccardi \& Amorim (submitted) 
68. Scutum area, external line to the dorso-lateral to dc (Figure 69a): (0) dark; (1) yellow.

69. Scutum area, external line to the ventro-lateral to dc (Figure 69a): (0) dark; (1) yellow.

70. Scutum anterior half (Figure 69b): (0) dark; (1) yellow.

71. Scutum area posterior half (Figure 69b): (0) dark; (1) yellow.

72. Propleuron (Figure 70a-b): (0) well developed; (1) weakly developed.

73. Postpronotal seta (Figure 71a-b): (0) 0 or 1 ; (1) 2 or more. Riccardi \& Amorim (submitted), Mlynarek \& Wheeler 2018

74. Postpronotal seta orientation, in case of 2 or more setae (Figure 72a-b): (0) only lateroclinate; (1) reclinate and lateroclinate.

75. Postpronotal lobe (Figure 73a-b): (0) mostly yellow; (1) mostly brown or black.

76. Anepisternum (Figure 74a-b): (0) purinosity; (1) bare. Riccardi \& Amorim (submitted)

77. Anepisternum setaceous (Figure 75a-b): (0) present; (1) absent.

78. Anepisternum color (Figure 76a-c): (0) entirely yellow(ish); (1) entirely brown or black;

(2) mostly yellow with brown marketing ventrally.

79. Katepisternum color (Figure 77a-c): (0) entirely yellow(ish); (1) entirely brown or black; (2) mostly yellow with brown marketing.

80. Meron color (Figure 78a-c): (0) yellow or light brown; (1) brown or black; (2) yellow(ish), dark marketing.

81. Number of distinct anterior notopleural setae (Figure 79a-d): (0) 1 ; (1) 2 ; (2) more; (3) 0. Riccardi \& Amorim (submitted), Mlynarek \& Wheeler 2018

82. Number of distinct posterior notopleural setae (Figure 80a-c): (0) 1 ; (1) 2; (2) more. Riccardi \& Amorim (submitted), Mlynarek \& Wheeler 2018

83. Purinosity on postpronotal lobe (Figure 81a-b): (0) present; (1) absent.

84. Numbers of supraalars (Figure 82a-c): (0) $0 ;(1) 1 ;(2) 2$. Mlynarek \& Wheeler (2018)

85. Numbers of postalars (Figure 83a-c): (0) 1 ; (1) 2 ; (2) more. Riccardi \& Amorim (submitted)

86. Intrapostalar (Figure 84a-b): (0) absent; (1) present. Mlynarek \& Wheeler (2018)

87. Length of scutellum (Figure 85a-c): (0) longer than wide; (1) wider than long; (2) almost as long as wide. Mlynarek \& Wheeler (2018)

88. Tip of scutellum in dorsal view (Figure 86a-b): (0) apically rounded or slightly pointed; (1) apically straight (flattened).

89. Laterals of scutellum in dorsal view (Figure 87a-b): (0) convex; (1) straight. 
90. Scutellar finger-like tubercles (Figure 88a-c): (0) absent; (1) present, only apicals; (2) present, apicals and subapicals. Mlynarek \& Wheeler (2018)

91. Scutellar large setae position (Figure 89a-b): (0) marginal; (1) ventral.

92. Scutellum color (Figure 90a-d): (0) entirely dark brown or black; (1) entirely yellow or light brown; (2) apically and laterally yellow or brown; (3) black or dark brown anteriorly; (4) laterally yellow or light brown; (5) basally yellow or brown; (6) apically and basally yellow or brown; (7) laterally black or dark brown; (8) yellow or light brown anteriorly.

\section{Wing}

93. Wing length compared to the abdomen length (Figure 91): (0) longer or as long; (1) shorter. Mlynarek \& Wheeler (2018)

94. Costal (Figure 92a-d): (0) ending at $R_{4+5} ;(1)$ ending at $M_{1+2} ;(2)$ ending between $R_{4+5}$ and $\mathrm{M}_{1+2} ;$ (3) ending before $\mathrm{R}_{4+5}$. Riccardi \& Amorim (submitted)

95. Anal lobe (Figure 93a-b) (0) present; (1) absent. Riccardi \& Amorim (submitted), Mlynarek \& Wheeler, 2018

96. Alula (length/width) (Figure 94): (0) lesser than 3; (1) between 3 to 5; (2) more than 5.

97. Pattern on the wing, apically (Figure 95): (0) translucent; (1) marked. Mlynarek \& Wheeler (2018)

98. Pattern on the wing, basally (Figure 95): (0) translucent; (1) marked.

99. Pattern on the wing, medially (Figure 95): (0) translucent; (1) marked.

100. Shape of pattern on the wing, apically (Figure 96): (0) restricted between veins; (1) unrestricted.

101. Basal radial cell relating to the basal medial cell (Figure 97a-b): (0) reaches before middle of basal medial cell; (1) at least at the medium of basal medial cell. Riccardi \& Amorim (submitted)

102. Height of basal radial cell (Figure 98): (0) no modification; (1) projecting dorsally.

103. Sinusoidal of CuA (Figure 99a-b): (0) absent (straight or slightly inward); (1) present (notably inward). Riccardi \& Amorim (submitted)

104. $R_{1}$ (Figure 100a-b): (0) slightly distally; (1) with a short bump.

105. Sc (Figure 101a-c): (0) almost entirely independent from $R_{1}$; (1) distinct from $R_{1}$ basally; (2) entirely indistinctive. 
106. Costal break on Sc part (Figure 102a-b): (0) present; (1) absent. Riccardi \& Amorim (submitted)

107. Costal break on humeral part (Figure 103): (0) present; (1) absent. Riccardi \& Amorim (submitted)

108. Shape of anterior end of $R_{2+3}$ (Figure 104a-b): (0) curved to the $C ;(1)$ about straight to the $\mathrm{C}$.

109. Shape of basal half of $\mathrm{M}_{1+2}$ (Figure 105): (0) convex anteriorly; (1) straight.

110. Shape of dm-cu (Figure 106): (0) convex; (1) straight.

111. Position of $\mathrm{dm}$-cu in relation to $\mathrm{CuA}_{1}$ (Figure $\left.107 \mathrm{a}-\mathrm{b}\right)$ : (0) more than $100^{\circ} ;(1)$ about $90^{\circ}$ or less.

112. C2 (Figure 108a-c): (0) at most reaches to the middle of the wing; (1) between middle and tip of the wing; (2) at the tip of the wing. Riccardi \& Amorim (submitted)

113. C4 (Figure 109a-b): (0) ending below the apical margin of the wing; (1) ending at the apical margin of the wing.

114. $M_{1+2}$ thickness (Figure $\left.110 a-b\right)$ : (0) as thick as $R_{4+5}$; (1) thinner than $R_{4+5}$. Riccardi \& Amorim (submitted)

115. Halter color (knob) (Figure 111a-b): (0) black or dark brown; (1) yellow-ish or light brown. Riccardi \& Amorim (submitted)

116. Halter color (stem) (Figure 111a-b): (0) black or dark brown; (1) yellow-ish or light brown.

Legs

117. Male mid femoral organ (Figure 112): (0) present; (1) absent. Mlynarek \& Wheeler (2018)

118. Tibial organ (Figure 113): (0) absent; (1) present; (2) not developed well. Riccardi \& Amorim (submitted)

119. Hind tibial spur (Figure 114a-b): (0) shorter or slightly longer than hind tibia diameter; (1) clearly longer; (2) absent. Mlynarek \& Wheeler (2018)

120. Hind tibia color: (0) yellow or light brown entirely; (1) black or dark brown entirely; (2) dorsal tip yellow, the rest dark; (3) dorsal and ventral tips yellow, the rest dark; (4) dorsal and ventral tips dark, the rest yellow; (5) around tibial organ dark, the rest yellow; (6) dorsally, medially and ventrally yellow, the rest dark. 
121. Hind tibial organ: (0) dark color; (1) yellow or white.

122. First hind tarsal segment: (0) yellow or light brown; (1) black or dark brown; (2) dorsally yellow, ventrally dark.

123. Fore tibia color: (0) yellow or light brown entirely; (1) black or dark brown entirely; (2) small part at the middle dark, the rest yellow; (3) dorsal tip yellow, the rest dark; (4) dorsally, medially and ventrally yellow, the rest dark.

124. First fore tarsal segment: (0) yellow or light brown entirely; (1) black or brown; (2) dorsal tip yellow, the rest dark.

125. Fore coxa color: (0) yellow or light brown entirely; (1) black or dark brown entirely; (2) ventral half yellow, dorsal half dark; (3) dorsal and ventral tip yellow, the rest dark; (4) ventral tip yellow, the rest dark.

\section{Abdomen}

126. Abdominal tergites (Figure 115): (0) no modification; (1) syntergites $1+2$ expanded comparing with other tergites. Mlynarek \& Wheeler (2018)

127. Sclerotization of tergite (Figure $116 a-b)$ : (0) syntergites $1+2$ slightly sclerotized; (1) syntergites $1+2$ firmly sclerotized; (2) syntergites $1+2$ and tergite 3 firmly sclerotized. Mlynarek \& Wheeler (2018)

128. Color of 5th tergit of abdomen: (0) entirely yellow; (1) entirely black or dark brown; (2) dark in 2 or 3 vertical lines; (3) anteriorly yellow, posteriorly dark; (4) anteriorly dark, posteriorly yellow.

129. Color of $1^{\text {st }}$ tergit of abdomen: (0) entirely yellow; (1) entirely black or dark brown; (2) dark in 1 to 3 vertical lines; (3) anteriorly yellow, posteriorly dark; (4) ventral laterals dark.

\section{Male terminalia}

130. Syntergosternite 7+8 (Figure 117): (0) broad; (1) narrow.

131. Epandrium medial line (dorsal sulcus) (Figure 118): (0) present; (1) absent.

132. Antero-ventral corner of epandrium in posterior view (Figure 119a-b): (0) with a short projection; (1) with a long projection; (2) absent. Riccardi \& Amorim (submitted)

133. Size of surstylus (comparing with the size of epandrium) (Figure 120a-b): (0) short; (1) long. Riccardi \& Amorim (submitted), Mlynarek \& Wheeler 2018 
134. Elongated setae at the edge of anterior lateral margins of surstylus (Figure 121a-b): (0) present; (1) absent. Riccardi \& Amorim (submitted)

135. Elongated setae at lateral margins of surstylus modified to spines (Figure 122): (0) present; (1) absent.

136. Marginals of surstylus in posterior view (Figure 123a-b): (0) straight; (1) indentured. Riccardi \& Amorim (submitted)

137. Anterior and posterior lateral margins of surstylus (Figure 124a-d): (0) parallel; (1) convergent; (2) divergent. Mlynarek \& Wheeler (2018)

138. Location of cercus on epandirum (Figure 125a-b): (0) placed on proctiger, free; (1) below proctiger, connected with epandrium. Riccardi \& Amorim (submitted)

139. Cercus articulation (Figure 126a-b): (0) articulated; (1) fused to epandirum.

140. Length of cercus (Figure 127a-e): (0) almost vestigial, in the middle; (1) almost vestigial, in laterals; (2) small; (3) enlarged, broad; (4) enlarged, narrow. Riccardi \& Amorim (submitted), Mlynarek \& Wheeler 2018

141. Elongated setae at tip of cercus (Figure 128a-c): (0) absent; (1) present, 1 to 3; (2) present, several. Mlynarek \& Wheeler (2018)

142. Cerci (Figure 129a-d): (0) separated from each other; (1) fused basally or until midway to apex; (2) fused entirely or more than midway broadly; (3) fused entirely narrowly (mesolobe). Mlynarek \& Wheeler (2018)

143. Proctiger (Figure 130a-b): (0) projected dorsally; (1) almost flat, not visible laterally.

144. Proctiger in posterior view (Figure 131a-c): (0) elongated dorsoventrally; (1) widened laterally; (2) almost as long as wide.

145. Shape of proctiger in lateral margins (posterior view) (Figure 132a-c): (0) concave; (1) almost $90^{\circ} ;(2)$ almost straight.

146. Ventral margin of proctiger (Figures 132c, 133): (0) concave; (1) straight.

147. Sub-anal plate (under proctiger) (Figure 134a-b): (0) present; (1) absent. Riccardi \& Amorim (submitted)

148. Hypandrium (Figure 135): (0) firmly connected to epandrium, articulated outside of epandrium; (1) sclerotized simply, articulated inside.

149. Shape of hypandrium (Figure 136a-b): (0) arms not connected posteriorly; (1) arms connected posteriorly. Riccardi \& Amorim (submitted) 
150. Phallapodeme length (Figures 136a, 137): (0) long; (1) short. Riccardi \& Amorim (submitted)

151. Base of phallapodeme (Figures 137, 138): (0) unbranched basally; (1) branched basally.

152. Phallapodem sclerite (Figure 139a-d): (0) absent; (1) developed only basally (branches are occupied by that); (2) developed only distally (branches are free); (3) developed basally and distally. Riccardi \& Amorim (submitted)

153. Length of endophalic process (Figure 140a-b): (0) developed; (1) vestigial.

154. Distiphallus (Figure 141a-b): (0) hairy; (1) bare. Riccardi \& Amorim (submitted)

155. Synchrony of S6 (Figure 142a-c): (0) asymmetrical; (1) symmetrical, frontally covers hypandrium; (2) absent. Riccardi \& Amorim (submitted)

156. Hypandrium, anterior view (Figure 143a-b): (0) not sclerotized ventrally; (1) sclerotized ventrally, divided it in 2 parts.

\section{Female terminalia}

157. Shape of segment 7 (Figure 144): (0) longer than wide; (1) as long as wide; (2) shorter than wide.

158. Shape of segment 8 (Figure 145): (0) three times or more longer than wide; (1) longer or as long as wide; (2) shorter than wide.

159. Sternite 8 (Figure 146a-b): (0) one piece; (1) V-shaped; (2) completely divided longitudinally in two pieces lateral sclerites.

160. Shape of sternite 8 (Figure 146a): (0) flattened at the segment 8 ; (1) protruding in 2 lobes anteriorly.

161. Cerci coming out: (0) separated distally; (1) close to each other distally.

162. Cerci (Figure 147): (0) not sclerotized; (1) sclerotized.

163. Shape of cerci (Figure 148a-c): (0) elongated, cylindral; (1) short, round; (2) short, triangular.

164. Hypoproct (Figure 149): (0) densely covered with microtrichia; (1) few microtrichia present; (2) entirely bare.

165. Hypoproct setation (Figure 150): (0) aligned at the frontal margin of hypandrium in row(s); (1) spread all over of the disk of hypoproct.

166. Epiproct (Figure 151): (0) with microtrichia; (1) entirely bare of microtrichia. 
167. Number of setae on epiproct (Figure 152a-b): (0) 2 in the centre of epiproct disk; (1) 3 in the centre of epiproct disk; (2) numerous and dispersal; (3) 0.

Of the complete list of characters, 101 are binary characters and 66 have three or more character states, in a matrix with 55 oscinelline species of 50 genera, and five rhodesielline species of three genera. The analysis under implied weight with $\mathrm{k}$ value $=3$ rendered one single maximum parsimonious tree (MPT) with 1984 steps (Figure 2). The Consistency index $(\mathrm{Ci})$ is 0.14 and the Retention index ( $\mathrm{Ri})$ 0.42. The analysis under implied weight using the setk.run script $(k=17.17770)$ also provided only one single most parsimonious tree, with 1903 steps and $\mathrm{Ci}=0.15$ and $\mathrm{Ri}=0.44$ (Figure 1 ).

As briefly discussed above, the use of successive posterior weighting of characters prevents the risk of highly plastic characters to establish nodes in the parts of the tree with less support from mutually congruent characters. The topology chosen as the best approach here, with default option in TNT with $\mathrm{k}=3$, enhances the weight of the features with nested congruence over incongruent characters. Our position is that, more important than exact topology of the trees obtained with the two different $k$ values, the comparison of between the trees helps detecting the rogue terminals and the larger stable clades in the tree.

There was an effort to extend the outgroup sampling within chloropids, rooting at Carnidae, although the character sampling at the level of the outgroups was beyond the scope of this study. Nevertheless, the discussion of the relationships between the subfamilies is relevant. There is a considerable evidence that the Siphonellopsinae is sister to the remaining chloropids. In this sense, the position of the Chloropinae as sister to the remainder of the family in our tree (Figure 2) seems to be due to undersampling of characters. The monophyly of (Rhodesiellinae + Oscinellinae), however, has clear support in the literature (Figures 2, 4, 5).

The monophyly of the Rhodesiellinae, with its tribes Rhodesiellini, Stenoscinini, and Scoliophthalmini, as proposed by Nartshuk (2012), was not recovered here. The results we obtained, however, shed light over the problem of the relationships between the Rhodesiellinae and the Oscinellinae. Rhodesiella indeed appears as a clade sister to all Oscinellinae (including the Stenoscinini). Stenoscinis and Psilacrum, on the other hand, do not fit together with Rhodesiella in a clade (Figure 5). We did not have samples of Scoliphthalmus, so for the time being we cannot solve the problem of its position. 
Stenoscinis belongs in a clade which is sister to all other oscinellines. The curve at the base of $\mathrm{M}_{1+2}$ vein of the wing define well a clade with Rhodesiella and close by genera (Figures 5 , 105). The overall delimitation of Rhodesiellinae in Nartshuk (2012), however, mix apomorphic features shared by Rhodesiellinae s.s. (i.e., Rhodesiellini) and Oscinellinae, and the plesiomorphic condition of features that are apomorphic for the core oscinellines. This helps to produce Rhodesiellinae as a grade at the base of the Oscinellinae.

Stenoscinis in our results fits into a clade together with Disciphus, Anatrichus and Rhopalopterum, which is sister to all remaining oscinellines (Figures 3,5 ). The position of this clade in the tree with $k=3$ differs from the position in the tree obtained from the setk script (Figures 1, 2). The generic composition of the clade, however, does not change, meaning that the clade itself is stable. It is supported by the presence of supercervial setae, an elongated palpus, the presence of purinosity on the anepisternum, the color of the first tarsal segment of the foretibia, and the shape of the female proctiger in posterior view (Figure 5). Hence, we keep the tribal status of Stenoscinini (for the time being with a different generic composition), but transferred back the tribe from the Rhodesiellinae to the Oscinellinae.

The position of Psilacrum was particularly puzzling. In none of our analyses it gathered either with Rhodesiella or with Stenoscinis, as it would first be expected (Figures 2, 8). This either means that the position of Psilacrum in the $\mathrm{k}=3$ tree is due to noise or that the position of Psilacrum is indeed not in the Rhodesiellinae or in the Stenoscinini, in such a way that non-homologous similarities between Psilacrum and Stenoscinis would have resulted in their approximation in the classification. We do not have now information at hand to answer this question. A wider study of the rhodesiellines will help to establish a clear picture of the position of the other genera. This includes particularly Scoliphthalmus, but also Javanoscinis, and the remaining genera associated to Rhodesiella.

Andersson's (1977) discussion on the Rhodesiellinae (as a tribe in his system) clearly supports the conclusions of this study at this level. He carefully discussed the features shared by oscinellines and rhodesiellines from a phylogenetic perspective (Andersson 1977, p. 55): "The Oscinellinae genera can be grouped into two groups which I think deserve the rank of tribes, viz. Rhodesiellini, which show many plesiomorphous features such as divergent ocellar setae, hairy eyes, hairy mesopleuron, and Oscinellini, with a higher 
number of apomorphous characters." Kanmiya (1983, p.32) agreed with this division and added some additional features of the male terminalia.

In other words, Andersson (1977) was already able to recognize apomorphic features that join these two main groups, but recognized that there are apomorphies of the core oscinellines which are not present in the Rhodesiellinae s.s., i.e., excluding Javanoscinis. Later on, Andersson says (p. 56), "The Rhodesiellini undoubtedly belongs to Oscinellinae but is quite distinct from Oscinellini. The plesiomorphous feature, divergent procurved oc is present in both Chloropinae and Siphonellopsinae but not in Oscinellini except in the odd genus Javanoscinis. On the other hand the apomorphous feature, femoral organ is present also in Oscinellini". This means that he even recognized that there are apomorphic features in Javanoscinis (what also applies to Stenoscinis, a genus he did not examine) that do not strictly fit into a clade with Rhodesiella. Kanmiya $(1983$, p. 64$)$ had a species of Stenoscinis in his study. He included the genera Stenoscinis and Psilacrum (Oscinoides s.l.) together with Javanoscinis into the "Oscinellini".

The presence of Anatrichus and Disciphus within the Stenoscinini is confusing. Andersson (1977, p. 66) and Kanmiya (1983) include them in the Elachiptera genus group, a position followed by Mlynarek \& Wheeler (2012). We hardly would have any doubt about their connection to the Elachipterini, and we understand that possibly decisions concerning character coding may have displaced these two genera. Sabrosky (1980) mentioned that Rhopalopterum corresponds to Lioscinella by Andersson (1977) and that Nearctic species associated to the genus have been previously placed in Stenoscinis. Hence, there is a chance that Rhopalopterum is either part of this clade with Stenoscinis, though deserving its generic rank, or is a relatively plesiomorphic genus of core oscinellines.

With the results of this study (Figure 2), both kinds of ranking at this level would work-a single subfamily Oscinellinae, with two main subclades, as in Andersson's (1977) system, or two sister subfamilies, Rhodesiellinae and Oscinellinae, as in Nartshuk's (2012) system. We prefer here to keep the subfamily status given to the Rhodesiellinae, with more space for a detailed tribal classification within the oscinellines. The basic difference with Nartshuk's (2012) system about the Rhodesiellinae is that we transfer back the Stenoscinini to the Oscinellinae, where it fits as a clade sister to the core oscinellines. Mlynarek \& Wheeles's (2018) study of the Elachipterini had a sampling of outgroups with eight oscinelline genera, what does not warrant a safe test of the hypothesis of monophyly of the 
tribe. They found, within their composition of the Elachipterini, a clade sister to the remaining members of the tribe composed by the genera Disciphus, Allomedeia, Alombus, Sepsidoscinis and Anatrichus. The other clade has the genera Goniaspis, Ceratobarys, Lasiochaeta, and Elachiptera. As mentioned ahead, our results corroborate the position of Sepsidoscinis close to Elachiptera. It is interesting to note that Anatrichus and Disciphus come together in the Stenoscinini.

The genera Goniaspis and Monochaetoscinella in our study (Figures 2, 6) compose a clade which is sister to all remaining oscinellines except the Stenoscinini. This suggests that these two genera are plesiomorphic for a number of important features in the core oscinellines and that they should not be part of the higher clades of the subfamily. Both these genera are unplaced at the tribal level in Nartshuk's (2012) system for the Oscinellinae. Their precise position, however, is not clear and we prefer, for the time being, to keep them incertae sedis, in the classification.

The relationships found here between the genera of the core oscinellines are not easy to interpret (Figure 2). One of the reasons is that 14 of the 53 genera included in our analysis were not included by Nartshuk's (2012) in any of the tribes; and 38 of the 66 genera included in her system could not be included in this study. Hence, of the 124 genera of Oscinellinae, only 38 are common to both studies.

A second reason for the complexity of the task is that the diagnoses of some of the tribes in Nartshuk's (2012) system are based on plesiomorphies or homoplasies. To bring together the entire breadth of the oscinellines into a single system is a major effort and a guide for the study of the group. Still, some of the tribes under her delimitation apparently do not correspond to clades. A final reason for the difficulty in interpreting the incongruence is that we are aware that this first approach still does not have the taxon density and density of characters to recover the entire robust clades.

The differences in the list of genera examined by Andersson (1977) and by Nartshuk (2012), with many genera missing in both systems, creates an even more difficult scenario to evaluate the results of this study, in the sense that there is not a common ground between authors in the literature.

In the group that we informally refer to as the core oscinellines (Figure 6), there is a clade including the type-species of Oscinella-Oscinella frit-that is sister to all oscinellines except Goniaspis and Monochaetoscinella and the Stenoscinini. The clade includes the 
genera Oscinella, Malloewia and Lipara. The generic composition of our Oscinellini, of course, is puzzling-supported by inner vertical setae, the rate of eye comparing with head capsule vertically, the shape of the anterior end of $R_{2+3}$, the color of the stem of halter and the length of endophallic process. Lipara has a broad facial carina (Figure 51a), which is not typical of Oscinella s.s. These features are highly homoplastic and may have gathered genera not indeed nor directly related. The Oscinella genus group of Andersson (1977) also includes Tropidoscinis (not analysed in this study), Lioscinella and Hapleginella. The Oscinellini of Nartshuk (2012) include Oscinella and the genera Camptoscinella Sabrosky (removed from the matrix due to amount of missing data), Conioscinella Duda, Olcella Enderlein (not analysed here), Oscinimorpha Lioy, Neolcella Cherian (also not analysed), and Neoscinella Sabrosky. Malloewia, not present in Andersson`s (1977) and Kanmiya`s (1983) studies, integrates the Incertellini sensu Nartshuk (2012).

This position of Oscinella in the tree (Figure 6), however, seems consistent: the diagnosis of the Oscinella genus group of Andersson (1977) is largely composed of plesiomorphies, what may explain why the genera gathered in Andersson's (1977) Oscinella genus group also have a relatively basal position in the clade corresponding to the core oscinellines. In other words, the genera Tropidoscinis, Hapleginella, and Lioscinella are clearly plesiomorphic for features in higher oscinellines, as in the enlarged Elachipterini clade or in the Siphonellini / Oscinisomatini / Fiebrigellini clade.

Our clade Siphonellini/Oscinisomatini/Fiebrigellini/Tricimbini corresponds to a rather large group including the genera Calamoncosis, Chaetochlorops, Epimadiza, Eribolus, Fiebrigella, Hapleginella, Heteroscinis, Oscinisoma, Loxobathmis, Polyodaspis, Siphonella, Siphunculina and Tricimba (Figure 6). This clade is supported by the color of the first antennal flagellomere (which is homoplastic) and the height of the basal radial cell (dorsally projected), which is an apomorphy unique to this group in the tree (Figure 98).

Hapleginella is included by Andersson (1977) and Kanmiya (1983) in the Oscinella group, while Nartshuk (2012) includes it in the Botanobiini. This conflict is quite revealing of the doubts about position of the genus. Independently of the question of its precise position, Hapleginella is clearly plesiomorphic for modified features in the higher oscinellines, so it is most certainly not a Botanobiini. Calamoncosis is placed in the Lipara genus group by Andersson (1977) and Kanmiya (1983), and in the Liparaini by Nartshuk 
(2012), so its position in the clade with genera of Siphonellini, Oscinisomatini, and Fiebriegellini should be dismissed as due to noise in the analysis.

Most of the other genera in this clade belong to the Fiebrigellini of Nartshuk (2012)-Chaetochlorops, Epimadiza, Fiebrigella, Heteroscinis, Polyodaspis-, meaning that there is a considerably large overlap between Nartshuk's (2012) delimitation of the tribe and this clade. The clade, however, also includes genera of other tribes, as Hapleginella (Botanobiini), Tricimba (Tricimbini), Oscinisoma (Oscinisomatini), Calamoncosis (Liparaini), and Siphonella (Siphonellini). Considering the conflicts between the generic composition of the supra-generic taxa in the systems of Andersson (1977) and Nartshuk (2012), and the amount of plesiomorphies and homoplasies in the diagnoses of these groups, it is hard to assess how robust or how weak is our clade.

The Lipara group of Andersson (1977) includes Lipara, Calamoncosis and Siphonella, while the Polyodaspis genus group of Andersson (1977) includes most of Nartshuk's (2012) genera of Fiebrigellini. Sabrosky (1941, p. 749) already had mentioned that "there is considerable doubt about the relation of Calamoncosis to Gymnopoda s.l." (in Nartshuk 2012 synonimyzed as Lipara), which finds support in our results. A separation between Lipara and Calamoncosis and a connection between Calamoncosis and Siphonella both find support in the literature.

Andersson (1977, p. 83) specifically mentioned, however, that Pseudeurina "differs from the other genera in having a yellow ground color, bare frontal triangle, erect, cruciate $o c$ and no tibial organ". He lists features to justify the inclusion of the genus in the Lipara genus group, but this brings some support that Pseudeurina in some degree has differences from the core Liparaini. Because Calamoncosis and Siphonella are clearly plesiomorphic in relation to feature typic of the higher oscinellines, which is also the case of Lipara, it would not be out of place admitting that the Liparaini has a diagnosis that brings together non related genera. In this case, Pseudeurina would definitely not belong with Lipara in a smaller clade with tribal status.

Kanmiya (1983) proposed a Siphonella genus group excluding Lipara, while Nartshuk (1983) transferred all members of the Lipara group to a new genus group, Siphonella, but Nartshuk (2012) followed Kanmiya's (1983) position and her Liparaini excludes Siphonella.

Tricimba, recovered in our tree as a member of this clade that also has Siphonella, Fiebriegella, Oscinisoma, and some other genera (Figure 2), is one of the large stable 
oscinelline clades in our study. Tricimba is a cosmopolitan genus, with a large interspecific variation. In his excellent study of Tricimba and Aprometopis, Ismay (1993, p. 313) compared the tribes of Incertellini and Tricimbini, arguing that the delimitation of these groups in the literature at that time was not accurate. He mentioned “(...) to maintain the distinction between the two tribes requires many cases of apomorphic characters having reverted to the plesiomorphic state." Ismay (1993) synonimyzed Nartshukiella to Tricimba, and Tricimbomyia and Pseudotricimba were placed in Tricimbini. In Ismay's (1993) system, Aphanotrigonum, Incertella, Meijerella, Apallates, Biorbitella, and Malloewia compose the Incertellini.

In our tree, Aphanotrigonum is not in the Siphonellini / Oscinisomatini / Fiebrigellini / Tricimbini clade (Figure 3), but is rather a rogue genus floating off the Dicraeini / Botanobiini / Elachipterini clade (Figure 3), as also happens with Apallates, Biorbitella and Incertella. Andersson (1977, p. 96) mentions that his Aphanotrigonum genus group (that also includes Tricimba and Siphunculina) shares modified features with the groups Lipara and Polyodaspis, and species in Siphunculina had been part of Fiebrigella in the past. Oscinisoma is part of the Eribolus genus group in Andersson's (1977) system - both genera belonging to the Oscinisomatini of Nartshuk (2012). Andersson (1977, p. 113) mentions that the Eribolus genus group has features of the male terminalia similar to the Oscinella genus group, suggesting that there are shared plesiomorphies between these groups. Meijerella, that belongs to the Aphanotrigonum genus group of Andersson (1977), was removed from the matrix in our study due to missing data.

There are 12 genera and one additional species of Oscinella that do not fit either in the Siphonellini / Oscinisomatini / Fiebrigellini / Tricimbini clade (Figure 3), nor in the Elachipterini / Botanobiini / Dicraeini clade (Figure 3). They are rather rogue genera, in the sense that their position vary in the tree (Figures 3,7 ) with changes in the $k$ value. The reason is is probably that there is not enough character sampling for this area of the tree to bring stability and reliability to clades. In other words, there is some information concerning these genera in terms of shared apomorphies and plesiomorphies, but their position in our tree is not reliable as indication of their precise relationships.

In terms of Nartshuk's (2012) system, Speccafrons belongs to the Siphonellini, Lasiambia belongs to the Fiebrigellini, Lioscinella and Hippelates belong to the Hippelatini, Oscinella cf. flavitibia, Conioscinella and Oscinimorpha belong to the Oscinellini, and 
Apallates, Biorbitella, Aphanotrigonum, Incertella, Microcercis and Neoscinella belong to the Incertellini. In a certain way, this suggests that Nartshuk's (2012) concept of Incertellini may indeed correspond to a grade relative to the higher Elachipterini.

It is worth mentioning that Apallates and Biorbitella compose together a small clade, reinforcing the positions in the literature. This clade is supported by eight characters, including the ground color of frons, the position of arista, the size of the surstylus, the length of the phallapodeme, and the shape of segment 7 in the female terminalia. Apallates and Biorbitella integrate Ismay's (1993) and Nartshuk's (2012) Incertellini, but neither genera were examined by Andersson (1977). Speccafrons, in Andersson's (1977) and Kanmiya's (1983) studies, are placed in the Siphonella genus group. Nartshuk (1983) included Speccafrons in the Incertellini, while Nartshuk (2012) later transferred the genus back to the Siphonellini. Our tree is not able to provide proper clues for the position of Speccafrons. The genera belonging to the Oscinellini, the Fiebrigellini, Hippelatini, and the Siphonellini either may have been incorrectly included in other tribes or may have problems in their coding in our analysis and are consequently displaced in relation to their correct placement-impossible to say at this stage.

Despite the lack of resolution of the tree at this level, one important conclusion can be drawn concerning the Neotropical "group blanda" of Oscinella of Sabroski \& Paganelli (1984), to which Oscinella cf. flavitibia, studied here, and five other species belongs (Figure 7). Sabroski \& Paganelli $(1984$, p. 28$)$ stated that "[t]his small group of tiny species with linear genae and very large, polished black, convex-sided frontal triangle may deserve generic distinction but there is no available name and for the present it is left in Oscinella until adequate material is available for study." Our results clearly show that the Oscinella cf. flavitibia falls in a separate part of the oscinelline tree and corroborate Sabroski \& Paganelli (1984) original suspicion and indeed a taxon of generic rank should be raised for the species of the Neotropical blanda group of Oscinella.

There is a larger clade that we informally refer to here as the "higher oscinellines". It includes Elachiptera (type genus of the Elachipterini) and Sepsidoscinis (also an Elachipterini), Gaurax (type genus of Botanobiini) and Pseudogaurax (also a Botanobiini), Dicraeus (type genus of Dicraeini), two genera assigned by Nartshuk to the Liparaini (Pseudeurina and Paracalamoncosis), one genus assigned to the Hippelatini (Chaethippus), Psilacrum (formally associated to the Rhodesiellinae), and 11 genera with no placement in 
Nartshuk's (2012) system (Figure 8). This is an important clade in our analysis and is stable between the tree with $\mathrm{k}=3$ and the setk tree. Five homoplastic features support this clade: extension of the anterior end of the ocellar triangle on the frons, the color of the anepisternum, in most cases entirely yellow with a black mark ventrally, the color of katepisternum, entirely yellow in most species, and the hypoproct setation in female terminalia.

This clade is particularly important as a result of the analysis. On the one hand, it is stable under different analysis parameters. On the other hand, it gathers 21 terminals of 18 genera, 11 of which are not present in Nartshuk's (2012) system. Of the seven genera common in both system (Figure 8), Elachiptera and Sepsidoscinis fit into the Elachipterini, Pseudogaurax and Gaurax belong in the Botanobiini, Chaethippus belongs in the Hippelatini, and Pseudeurina fits into the Liparaini, while Dicraeus is the only genus in the Dicraeini.

The generic composition of the Elachipterini in the cladistics analysis of the tribe in Mlynarek \& Wheeler's (2018) study was presented above. The character treatment of their analysis is very solid, but the complexity of the systematics of the Oscinellinae, with many potentially paraphyletic tribes, would demand a larger number of outgroups to properly test of the monophyly of the tribe. If we ignore the position of Chaethippus, the highest clade of this large group is basically of Elachipterini genera, with the presence of two additional, unplaced genera. Two Botanobiini genera, Gaurax and Pseudogaurax compose with these Elachipterini genera a more inclusive clade. And Dicraeus is sister to an even larger clade that gathers the Elachipterini / Botanobiini genera to two Liparaini genera, Pseudeurina and Paracalamoncosis, and a number of genera that are unplaced in Nartshuk's (2012) system.

The position of Dicraeus as sister to the entire remainder of the clade is not a problem. In Nartshuk's (2012) system it is a separate tribe Dicraeini, so there is no conflict of tribal composition. Nartshuk (1967) mentioned a possible relationships between Dicraeus with Aphanotrigonum and Tricimba. In our results, this would have support only on plesiomorphies. The basic question here is only whether or not the Dicraeini would be sister to the Elachipterini / Botanobiini clade.

The position of Psilacrum in our study, in this clade is very surprising. Our understanding is that there is some undetected noise that moved the genus from the Stenoscinini to this clade of core Elachipterini. Old World species of Psilacrum were dealt with by Ismay (1986, p. 19), where he states that “(...) Psilacrum may be placed in the 
Javanoscinis genus group of Andersson (1977) which also contains the widespread genus Stenoscinis."

One of the questions at this stage of the discussion, hence, from a phylogenetic perspective, is to which other tribes the Elachipterini, in its delimitation in Nartshuk (2012) and in Mlynarek \& Wheeler (2018), is associated, composing a larger clade. In other words, if some of the genera associated to other tribes - Liparaini, Botanobiini, and Hippelatinicould compose a larger monophyletic group with the Elachipterini.

In Mlynarek \& Wheeler's (2018) analysis, Cadrema was used as an outgroup. Andersson (1977, p. 65) in his discussion of the Elachiptera genus group mentioned: "Cadrema Walker has all the characteristic of the Elachiptera genus group; the habitus is similar, as are the genitalia". Kanmiya (1983, p. 67) created a genus group only for Cadrema. He mentions symplesiomorphic characters shared with the Siphonellopsinae. This shows that the position seems equivocate and hardly any feature will technically approach the genus from the siphonellopsines. It could be the case that some of the apomorphic features of Cadrema corresponds to secondary losses, but this does not affect the question of its position within the oscinellines. Nartshuk (1983) included Cadrema into the Hippelatinae s.l. (though the genus is unplaced in her 2012 system) and Andersson (1977) kept Cadrema under the Elachiptera genus group. The presence of Cadrema in this clade with Elachiptera, hence, should not be seen as a surprise. In this context, the presence of Hippelates as sister of the Dicraeini / Botanobiini / Elachipterini clade in the $k=3$ tree (Figure 8) is a corroboration of their position concerning the connection between Hippelates with Elachiptera and other higher oscinellines.

Pseudogaurax and Gaurax belong to the Gaurax genus group of Andersson (1977) and to the Botanobiini of Nartshuk (2012) (Figure 8). Andersson (1977, p. 76) indicates that the Gaurax genus group "is close to the Elachipterus genus group and the similarity of the species of the two groups is sometimes striking." He listed features that combine both groups-upright, cruciate $o c$, usually kidney-shaped or obliquely oval ant ${ }_{3}$, and densely haired or thickened arista, and well-developed tibial organs". Kanmiya (1983, p. 102) agrees with Andersson's (1977) proposal and noted "this genus group is not distinctly defined because of the lack of the autapomorphic characters". The genera Gampsocera and Cestoplectus, not examined here, also integrate the Gaurax genus group, while these two genera add to Gaurax Loew, Hapleginella Duda, Leucochaeta Becker, Pselaphia Becker, 
Pseudogaurax Malloch, Pterogaurax Duda in Nartshuk's (2012) Botanobiini. It is interesting to note that Andersson (1977) did not keep Hapleginella, as mentioned above, together with Gaurax, placing the genus in the Oscinella genus group (Figure 6). This probably means that the genus has important plesiomorphies concerning features that are modified in the Botanobiini (and closely related tribes in the higher oscinellines). Its placement in our tree, away from the other botanobine genera give some support to Andersson's (1977) position for this Hapleginella.

The genera Medeventor, Batrachomyia, Opetiophora, and Paracalamoncosis were proposed more recently and were not examined by Andersson (1977) and Nartshuk (2012). Wheeler (2007, p. 48) states that Medeventor "appears to be most closely related to the Old World genera Lipara Meigen and Pseudeurina de Meijere." Medeventor and Pseudeurina in our $\mathrm{k}=3$ tree (Figure 3,8 ) are very close to each other into this Elachipterini/Botanobiini clade. Lipara, as was already discuss, is plesiomorphic for the features in this higher oscinelline clade, and does not join Medeventor, Pseudeurina, and other genera. Indeed, features associating Medeventor and Pseudoeurina to Elachiptera seems based on a number of nested features. The fact that species of Pseudeurina comes out paraphyletic within this Elachipterini/Botanobiini clade seems to be the result of undersampling of characters at this level. The paraphyly of Medeventor in relation to Pseudeurina, however, seems reasonable (Figure 8).

There is nearly nothing about Opetiophora in the literature, a genus not examined by Andersson (1977) and not included in any tribe by Nartshuk (2012), but the proximity of Opetiophora with the Botanobiini/Elachipterini seems obvious. Batrachomyia has eleven described species, all from Australia and Papua New Guinea (Evenhuis 2006). It seems reasonable that the genus fit into this clade, but its position as sister of Opetiophora within the higher oscinellines may be subject to change as more characters and species are considered.

Paracalamoncosis was described very recently (Riccardi et al., 2018) as a genus of Liparaini based on a new Neotropical species similar to Calamoncosis-a genus known from all biogeographical regions except the Neotropics and the Australasian/Oceania. As we discussed above, the delimitation of the the Liparaini in Andersson (1977) and Nartshuk (2012) was not recovered here, Lipara falling into a clade with Oscinella frit. In our tree, Calamoncosis belongs in the clade with Siphonella and genera mostly of Fiebrigellini. In this 
context, it is hard to assess the question of the position of Paracalamoncosis. The tree topology suggests that the Liparaini is not monophyletic and that Paracalamoncosis is not related to Calamoncosis. It is conceivable that the presence of Paracalamoncosis in this larger Elachipterini clade may be due to noise.

Not much is available about the genera Trachysiphonella and Notaulacella, in such a way that not much can be said about its position in the tree in terms of other opinions on their taxonomic position in the classification. Finally, the position of Chaethippus, a genus placed by Nartshuk (2012) in the Hippelatini, is not entirely conflicting with the results here. Its precise position in the tree may be subject to changes within the clade in the future, but there is ground to accept its connection to the clade of the higher oscinellines (Figure 8). 


\section{Conclusions and perspectives}

This study is the first formal phylogenetic analysis for the Oscinellinae as a whole, based on 50 genera of the subfamily. Limited taxon sampling brings some analytical challenges, not only for the taxon not included the analysis, but also for the solution of some of the problems of homology, with risk of homoplastic features generating nodes. The number of characters, the use of analytical tools to reduce the influence of incongruent characters over nested characters, the systematic comparison of features across the subfamily, the number of outgroup levels used, and a careful consideration of information in the literature show that the phylogenetic signal obtained in this study is able to bring some phylogenetic order to the group.

Comparison between the trees obtained here under different analytical parameters helped to discern between stable clades and rogue taxa. Even though we do not intend to propose any formal changes to the classification of the subfamily at this stage, the tree has clades stable enough to corroborate comments in the literature about relationships among genera and about conflicting genera within the group. One of the important conclusions in the analysis is about the position of the Oscinellinae relatively to the remaining subfamilies and about the paraphyly of the Rhodesiellinae. The Rhodesiellinae with the delimitation including three tribes is shown here to be paraphyletic in relation to the Oscinellinae. The Stenoscinini, previously assumed to be a rhodesielline, is in a clade sister to all remaining Oscinellinae-informally referred to here as the core oscinellines.

Oscinella frit is considerably plesiomorphic for a number of features seen in other typical clades and has a relatively basal position in the tree. In other words, a clade that includes Oscinella frit seems to be sister of the set of core oscinelline genera. A larger, stable clade joins most of the Fiebriegellini to genera previously included in the tribes Siphonellini (Siphonella), Tricimbini (Tricimba and Siphunculina), Oscinisomatini (Oscinisoma), Liparaini (Calamoncosis), and Botanobiini (Hapleginella). It is clear that the Neotropical group blanda of Oscinella does not fit together with the type-species of the genus, corroborating previous indication in the literature that it needs a taxon of generic rank. A large group of genera that in Nartshuk's (2012) system belong to different tribes have an unstable position in the tree, but do not belong either in the Fiebriegellini / Siphonellini / Tricimbini / Oscinisomatini clade or in the Dicraeini / Botanobiini / Elachipterini clade. These rogue genera belong mostly to 
the Incertellini, but there are also genera that belong to the Hippelatini, Oscinellini, and Siphonellini. The diagnoses of these tribes are actually largely based on plesiomorphies and/or homoplastic features. Even not having the relationships between these genera clear, it is not surprising that genera from different tribes do not join the type genera of their respective tribes.

A large, stable clade informally referred to here as the higher oscinellines bring together the Dicraeini (Dicraeus) and genera of Botanobiini (Gaurax, the type-species of the tribe, and Pseudogaurax), Elachipterini (Elachiptera), Liparaini (Pseudeurina), and Hippelatini (Chaethippus). There is a good chance that Hippelates would compose a monophyletic group with this clade. Relevant information in the literature suggest that this group of genera indeed share apomorphic features and may compose a large clade in the subfamily.

It is still necessary to add a good number of the 74 genera of Oscinellinae to consider here into an integrated, formal phylogenetic analysis of the group to propose reliable changes in the classification of the subfamily. The results obtained here, however, brings considerable light to the understanding of the relationships in the Oscinellinae, clarifying the position of the Stenoscinini, indicating a relatively plesiomorphic condition of Oscinella frit, showing a large clade joining most of the Fiebriegellini to genera of the Siphonellini, Tricimbini, Oscinisomatini, Liparaini, and Botanobiini, and gathering in a higher oscinelline clade genera of the Dicraeini, Botanobiini, Elachipterini, and possibly of the Hippelatini. 


\section{References}

Andersson, H. 1977 Taxonomic and phylogenetic studies on Chloropidae (Diptera) with special reference to Old World genera. Entomologica scandinavica Supplementum, 8, 200pp.

Andersson, H. 1979. Problem vid kladistisk analys av flugfamiljen Chloropidae. Entomologisk Tidskrift, 100, 180-187.

Becker, T. 1910. Chloropidae. Eine monographische Studie. I. Teil. Paläarktische Region. Archivum zoologicum, 1(10): 33-174.

Brake, I. 2000. Phylogenetic systematics of the Milichiidae (Diptera, Schizophora). Entomologia Scandinavia 57 (Suppl.), 1-120.

Buck, M. 2006. A new family and genus of acalypterate flies from the Neotropical region, with a phylogenetic analysis of Carnoidea family relationships (Diptera, Schizophora). Systematic Entomology, 31: 377-404.

Cherian, P.T. and Emiliyamma, K.G. 1999. Description of a new species of Hapleginella Duda (Diptera: Chloropidae) from India. Zoological Survey of India, 97 (4): 167-170.

Chvála, M., Doskočil, J., Mook, J.H., and Pokorny, V. 1974. The Genus Lipara Meigen (Diptera, Chloropidae). Systematics, Morphology, Behavior, and Ecology. Tijdsch. Entomol, 117: 1-25.

Cumming, J.M. and Wood, D.M. 2009. Adult morphology and terminology. In: Brown, B.V., Borkent, A., Cumming, J.M., Wood, D.M., Woodley, N.E. \& Zumbado, M.A. (Eds.), Manual of Central American Diptera. Vol.1. National Research Council of Canada, Ottawa, pp. 2-50.

Deeming, J.C. and Al-Dhafer, H.M. 2012. Chloropidae from the Arabian Peninsula (Diptera: Cyclorrhapha). Zool. Middle East, 58: 1-88.

Duda, O. 1930a. Die neotropischen Chloropiden (Dipt.). Folia Zoologie und Hydrobiologie, 2, 46-128; Riga.

Duda, O. 1932. 61. Chloropidae. In: E. Lindner (Ed), Die Fliegen der Palaearktischen Region. Lieferungen 64, 48 pp, + I-III.

Evenhuis, N.L. 2006. First record of the frog parasite genus Batrachomyia Krefft from New Guinea (Diptera: Chloropidae). Zootaxa, 1351: 53-59. 
Goloboff, P.A. 1993. Estimating Character Weights during Tree Search. Cladistics, 9(1): 83 91

Goloboff, P.A., Farris, J.S. and Nixon K C. 2005. TNT: Tree analysis new technology. Systematic Biology, 54: 176-178.

Goloboff, P.A., Farris, J.S. and Nixon, K.C. 2008b. TNT, a free program for phylogenetic analysis. The Willi Hennig Society, Cladistics, 24: 774-786

Ismay, J.W. 1986. New Psilacrum from the Old World (Diptera, Chloropidae). Esakia, 24: 1935.

Ismay, J.W. 1993. Revision of Tricimba Lioy and Aprometopis Becker (Diptera: Chloropidae) from Australia and the Papua Region. Invertebrate Taxonomy, 7: 297-499.

Ismay, J.W. 2014. Developments in the genera of Chloropidae. In: 8th International Congress of Dipterology, Potsdam, pp. 148.

Ismay, J.W. and Nartshuk, E.P. 2000. Family Chloropidae. In: Papp, L. \& Darvas, B. (Eds.), Contributions to A Manual of Palaearctic Diptera (with special reference to flies of economic importance). Appendix Volume. Science Herald Press, Budapest, pp. 387429.

Kanmiya, K. 1983. A systematic study of the Japanese Chloropidae (Diptera). Memoirs of the Entomological Society of Washington, 11: 1-370.

Kanmiya, K. 1994. Studies on the eye-flies Siphunculina Rondani from Nepal (Diptera: Chloropidae). Jpn. J. Sanit. Zool., 45: 55-69.

Kubik, S. and Bartak, M. 2010. Anatrichus bimaculatus sp. n. (Diptera: Chloropidae) and differential characters between A. bimaculatus sp. n. and A. erinaceus. Biologia, 65(2): 330-332

Lipscomb, D. 1998. Basics of Cladistic Analysis. George Washington University, Washington D.C., USA, $75 \mathrm{pp}$.

Liu. X.Y, Nartshuk, E.P. and Ynag, D. 2017. Three new species and one new record of the genus Siphunculina from China (Diptera, Chloropidae). ZooKeys, 687: 73-88.

Malloch, J.R. 1913b. The genera of flies in the subfamily Botanobiinae with hind tibial spur. Proceedings of the United States national Museum, 46 (2024): 239-266.

Malloch, J.R. 1928. Notes on Australian Diptera. No. XIV. Proceedings of the Linnean Society of New South Wales, 53: 295-309. 
Malloch, J.R. 1938. Notes on Australian Diptera. No. XXXVII. Proceedings of the Linnean Society of New South Wales, 63: 334-356.

McAlpine, D.K. 1989. The taxonomic position of the Ctenostylidae (= Lochmostyliinae; Diptera: Schizophora). Memórias do Instituto Oswaldo Cruz, Rio de Janeiro, 84. Supl. IV: 365-371.

Mlynarek, J.J. and Wheeler, T.A. 2018. Phylogeny and revised classification of the tribe Elachipterini (Diptera: Chloropidae). Zootaxa, 4471(1): 1-36.

Mlynarek, J.J. and Wheeler, T.A. 2009. Revision of the Neotropical genus Goniaspis Duda (Diptera: Chloropidae). Zootaxa, 2033: 26-40

Nartshuk, E.P. 1967. Chloropid flies of the genus Dicraeus (Diptera, Chloropidae). Entomological Review, 46(2): 415-438.

Nartshuk, E.P. 1983. Classification of the superfamily Chloropoidea (Diptera, Cyclorrhapha), Cereal flies their system, evolution, and connection with plants. Entomological Review, 62(3): 180-193.

Nartshuk, E.P. 1987. Grass flies: Their System, Evolution and Relations with Plants. Tr. Zool. In-ta., 136: 1-279. [In Russian]

Nartshuk, E.P. 2006. Zoogeographical aspects of the North European fauna of Chloropidae (Diptera, Cyclorrhapha). Abstracts Volume of 6th International Congress of Dipterology, Fukuoka International Congress Center, Fukuoka, Japan, P. 176.

Nartshuk, E.P. 2012. A checklist of the world genera of the family Chloropidae (Diptera, Cyclorrhapha, Muscomorpha). Zootaxa, 3267, 1-43.

Nartshuk, E.P. 2014. Grass-fly larvae (Diptera. Chloropidae): diversity, habitats and feeding specializations. Entomological Review, 94 (4), 514-525. [translation of Russian text 2014 in Zoologicheskie Zhurnal, 93 (1), 81-91]

Nartshuk, E.P. and Andersson, H. 2013. The Frit Flies (Chloropidae, Diptera) of Fennoscandia and Denmark. Brill, Leiden, The Netherlands (Fauna Entomol. Scand. 43) vi + 282 pp.

Nartshuk, E.P., Smirnov, E.S. and Fedoseeva, L.I. 1988. Chapter 99, Family Chloropidae. In: Bei-Bienko, G.Ya. and Steyskal, G.C. (Eds.) Keys to the Insects of the European Part of the USSR. Smithsonian Institution Libraries and The National Science Foundation Press, Washington, D.C., USA. , pp. 667-731.

Nixon, C.K. 1999. Winclada (Beta) ver. 1.00.08. Published by the author, Ithaca, N.Y.

Nixon, C.K. and Carpenter, J.M. 1993. On outgroups. Cladistics, 9: 413-426. 
Paganelli, C.H. 2002. Revisão dos gêneros Neotropicais de Chloropinae (Incl. Mindidae). Fauna da Amazônia Brasileira, 24: 1-101.

Reilly, L.A., Favacho, J., Garcez, L. and Courtenay, O. 2007. Preliminary evidence that synanthropic flies contribute to the transmission of trachoma-causing Chlamydia trachomatis in Latin America. Cadernos de Saúde Pública, Rio de Janeiro, 23 (7): $1682-1688$.

Riccardi, P.R. 2016. Relações filogenéticas entre os gêneros da subfamília Chloropinae Rondani 1856 no mundo (Diptera: Chloropidae). Ph. D. thesis, Programa de PósGraduação em Entomologia, FFCLRP, Universidade de São Paulo, Ribeirão Preto, São Paulo.

Riccardi, P.R. \& Amorim, D.S. Submitted. Phylogenetic relationships of the Chloropinae Rondani 1856 of the world (Diptera: Chloropidae). Zoological Journal of the Linnean Society (submitted Dec 31, 2018).

Riccardi, P.R. and Ismay, J. 2017. First record of Psilacrum Becker 1912 (Chloropidae, Diptera) from Brazil with the description of a new species. Zootaxa, 4329 (5): 497500.

Riccardi, P.R., Bazyar, Z. and Lamas, C.E. 2018. New genus of the subfamily Oscinellinae from Brazil (Diptera: Chloropidae). Zootaxa, 4438 (2): 394-400.

Sabrosky, C.W. 1941. An annotated list of genotypes of the Chloropidae of the world (Diptera). Annals of the entomological Society of America, 34: 735-765.

Sabrosky, C. W. 1947. A revision of the African genus Epimadiza Becker (Diptera, Chloropidae). Annals and Magazine of Natural History, Ser. 11, 13: 821-851.

Sabrosky, C.W. 1951. Chloropidae. Ruwenzori Expedition 1934-1935. British Museum of Natural History, London, 2 (10): 711-828.

Sabrosky, C.W. 1980a. New genera and new combinations in Nearctic Chloropidae (Diptera). Proceedings of the entomological Society of Washington, 82(3): 412-429.

Sabrosky, C.W. 1984. (Draft) check list of the Chloropidae of the world (Diptera). Unpublished manuscript. $21 \mathrm{pp}$.

Sabrosky, C.W. and Paganelli, C.H. 1984. A Catalogue of the Diptera of the Americas South of the United States - Family Chloropidae. Museu de Zoologia, Universidade de São Paulo, 81:1-63. 
Schuh, R.T and Brower, A.V.Z. 2009. Biological Systematics: Principles and Applications. $2^{\text {nd }}$ Ed. Cornell University Press, 311 PP.

Spencer, K.A. 1978. Notes on the Australian Chloropidae (Diptera)-1. Stuttgarter Beiträge zur Naturkunde, (A) 309, 14 pp.

Walker, F. 1860. Catalogue of the Dipterous insects collected at Makessar in Celebes by $\mathrm{Mr}$ A. R. Wallace, with descriptions of new species. Journal and Preceedings of the Linnean Society (London), Zoology, 4: 145-172.

Wheeler, T.A. 2007. Two new genera of oscinelline Chloropidae (Diptera) from Costa Rica. Zootaxa, 1413: 47-53. 
Figures 


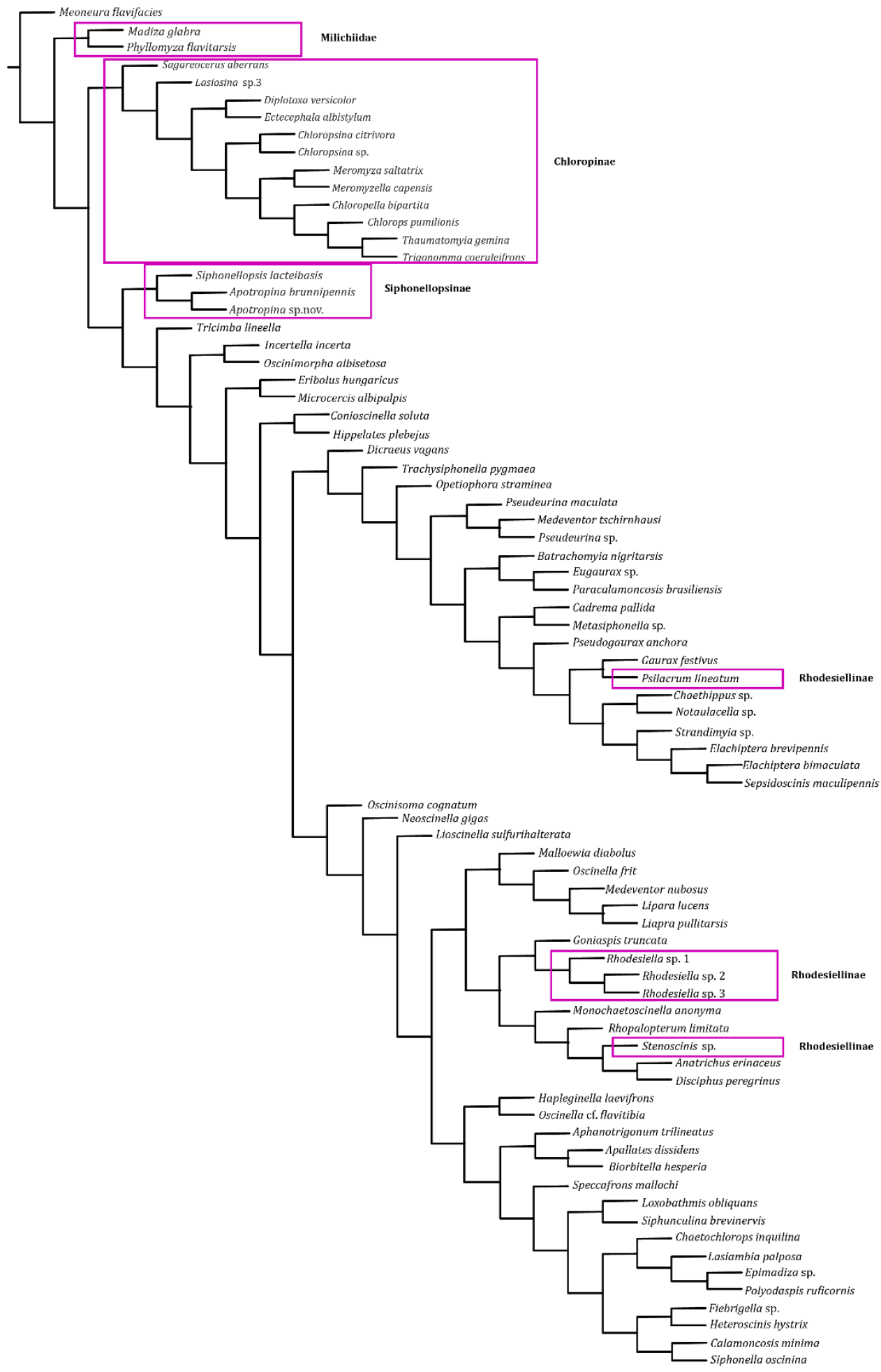

Figure 1. The single maximum parsimonious tree in phylogeny, under implied weighting scheme, setk script $=\sim 17, \mathrm{Ci}=$ $0.15, \mathrm{Ri}=0.44$. Marked areas, are representing the outgroups, and the position of rhodesielline genera in the obtained phylogeny. 


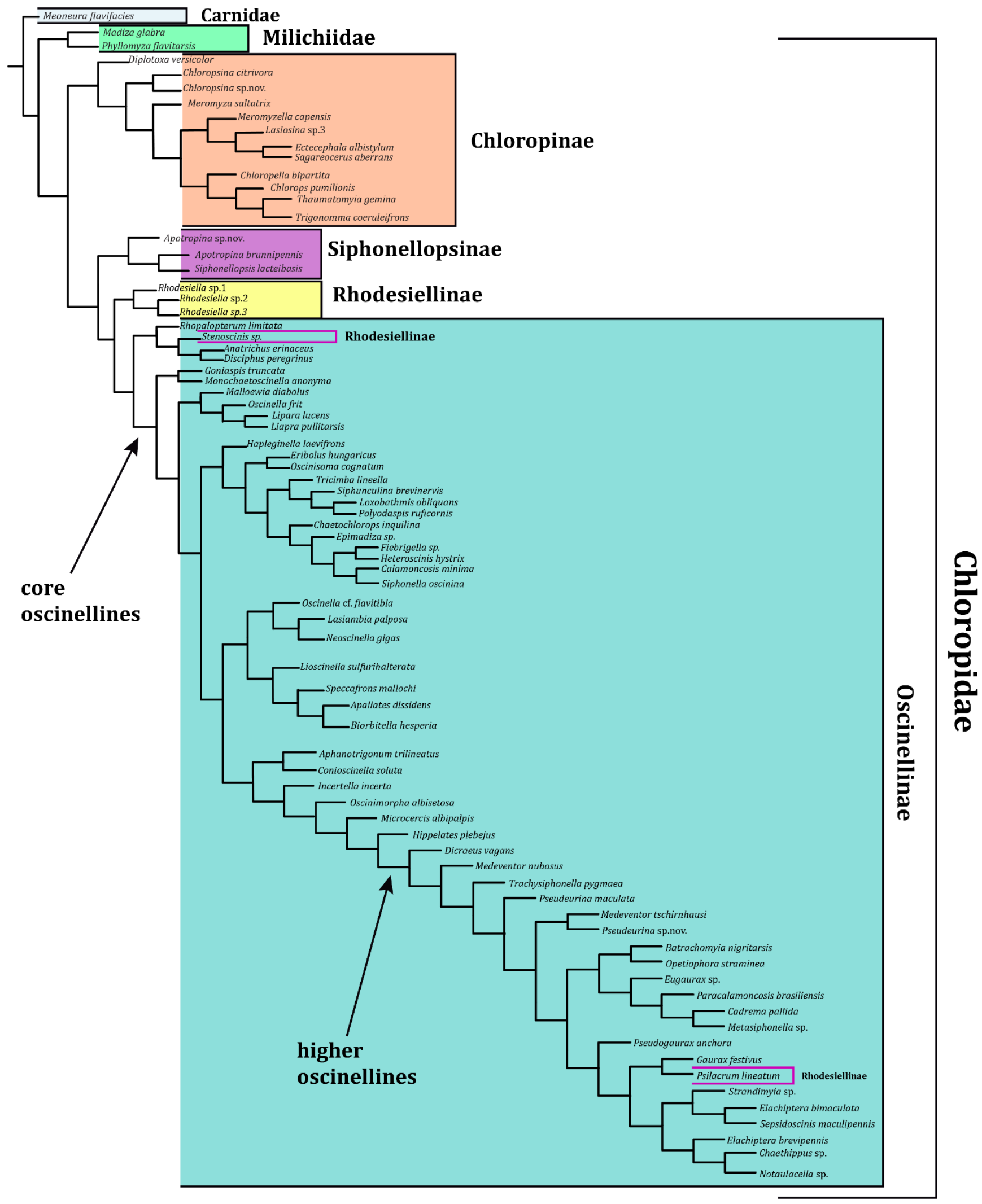

Figure 2. The single maximum parsimonious tree in phylogeny, under implied weighting scheme, $\mathrm{k}=3, \mathrm{Ci}=0.14, \mathrm{Ri}=0.42$. Marked areas are representing the outgroups and ingroups. The areas indicated with purple represent the position of rhodesielline genera in obtained phylogeny. 


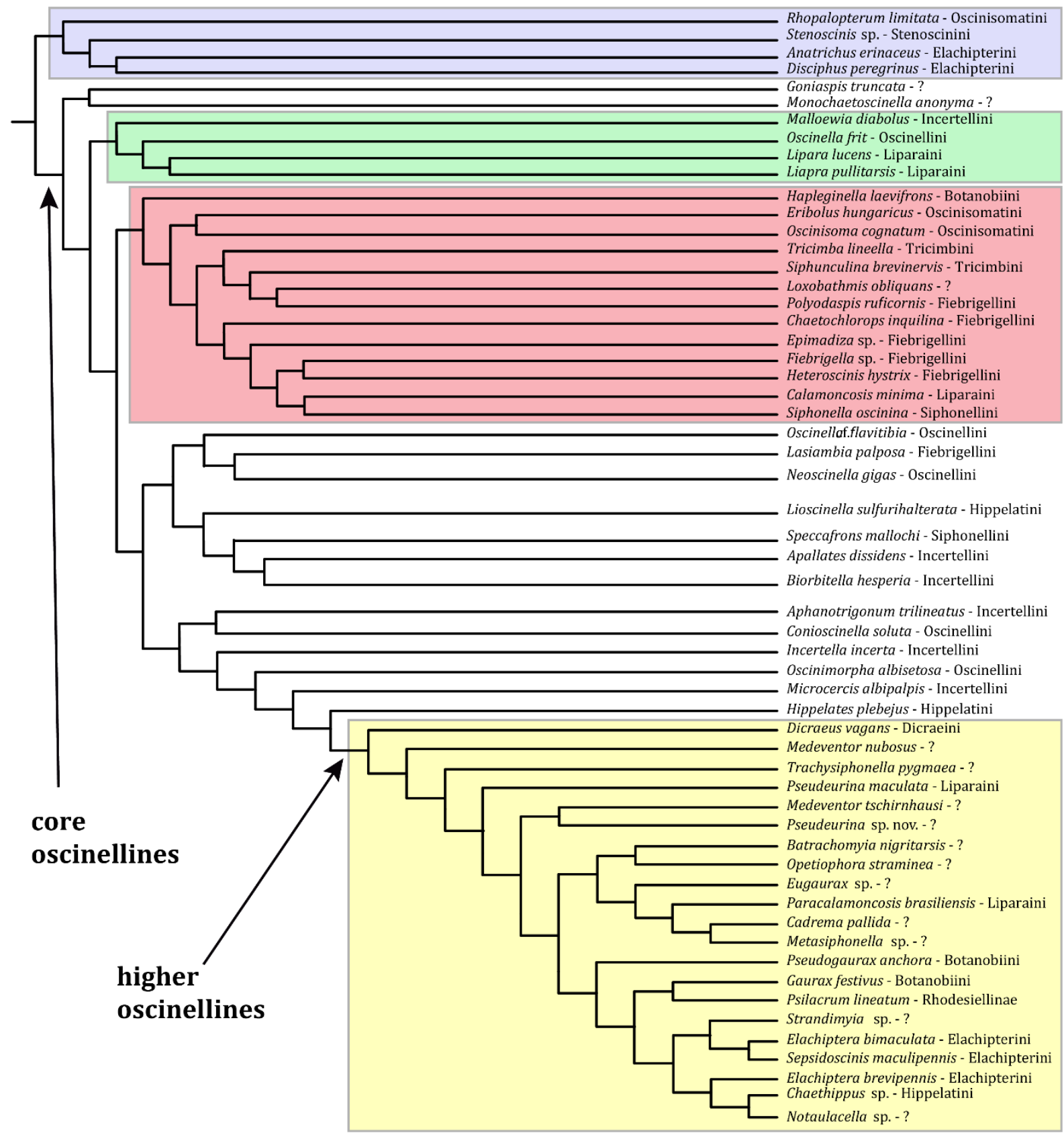

Stenoscinini

Oscinellini

Siphonellini+ Fiebrigellini+

Oscinosomatini + Tricimbini

Dicraeini+

Botanobiini+ Elachipterini

Figure 3. The phylogenetical relationship among the oscinellines (under implied weighting scheme, $\mathrm{k}=3$ ), with their tribal classifications in the previous literatures, front of each genus (Nartshuk 2012, Riccardi et al. 2018). 


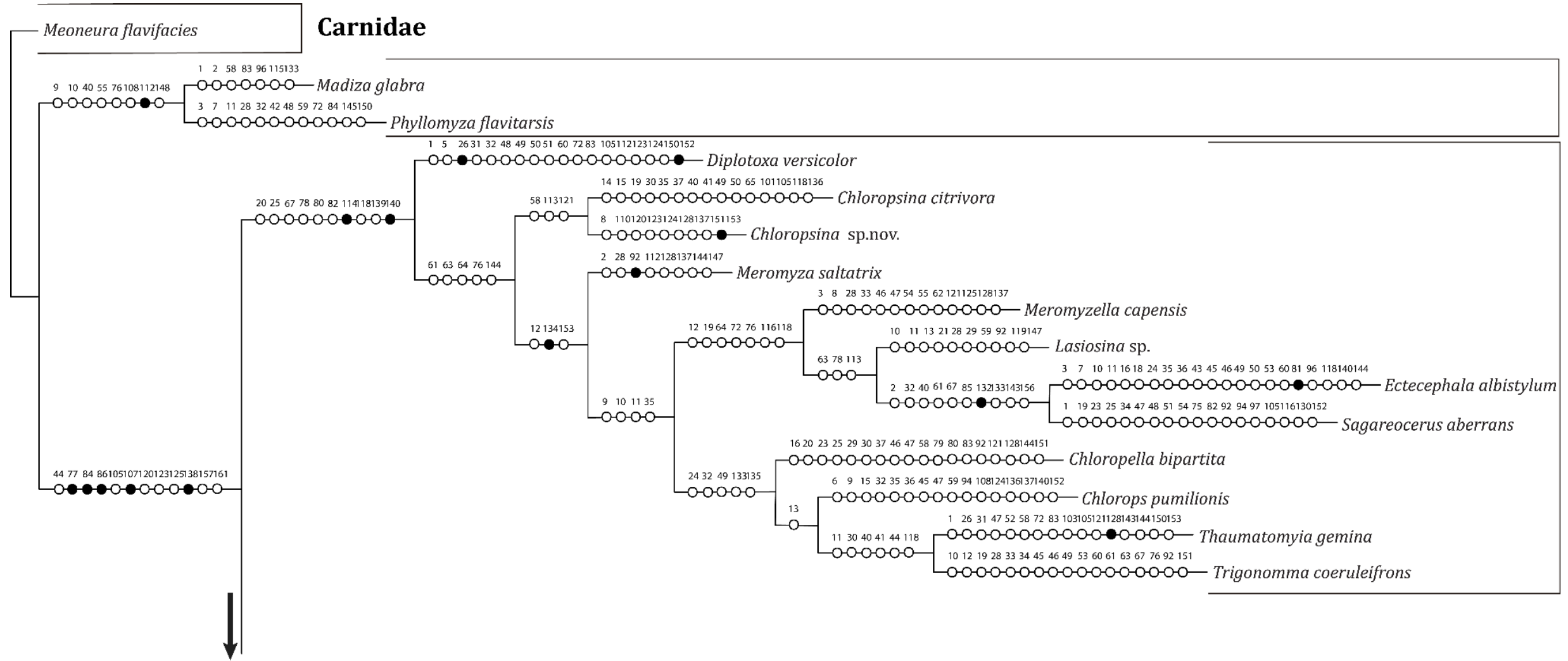

Milichiidae

Figure 4. The single maximum parsimonious tree in phylogeny, under implied weighting scheme, $\mathrm{k}=3, \mathrm{Ci}=0.14, \mathrm{Ri}=0.42 ;$ with unambiguous mapped on branches. White circles, homoplastic synapomorphies; full black circles, non - homoplastic synapomorphies. (Continuing) 


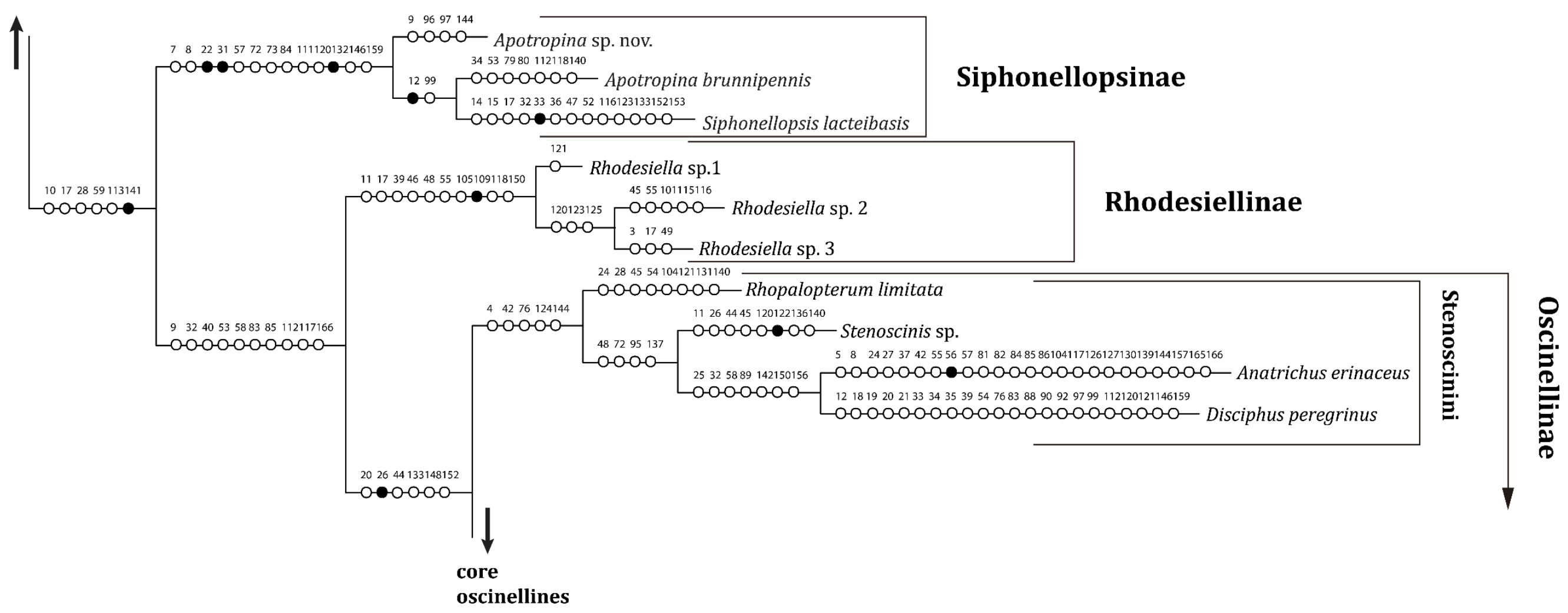

Figure 5. The single maximum parsimonious tree in phylogeny, under implied weighting scheme, $\mathrm{k}=3, \mathrm{Ci}=0.14, \mathrm{Ri}=0.42$; with unambiguous mapped on branches. White circles, homoplastic synapomorphies; full black circles, non - homoplastic synapomorphies. (Continuing) 


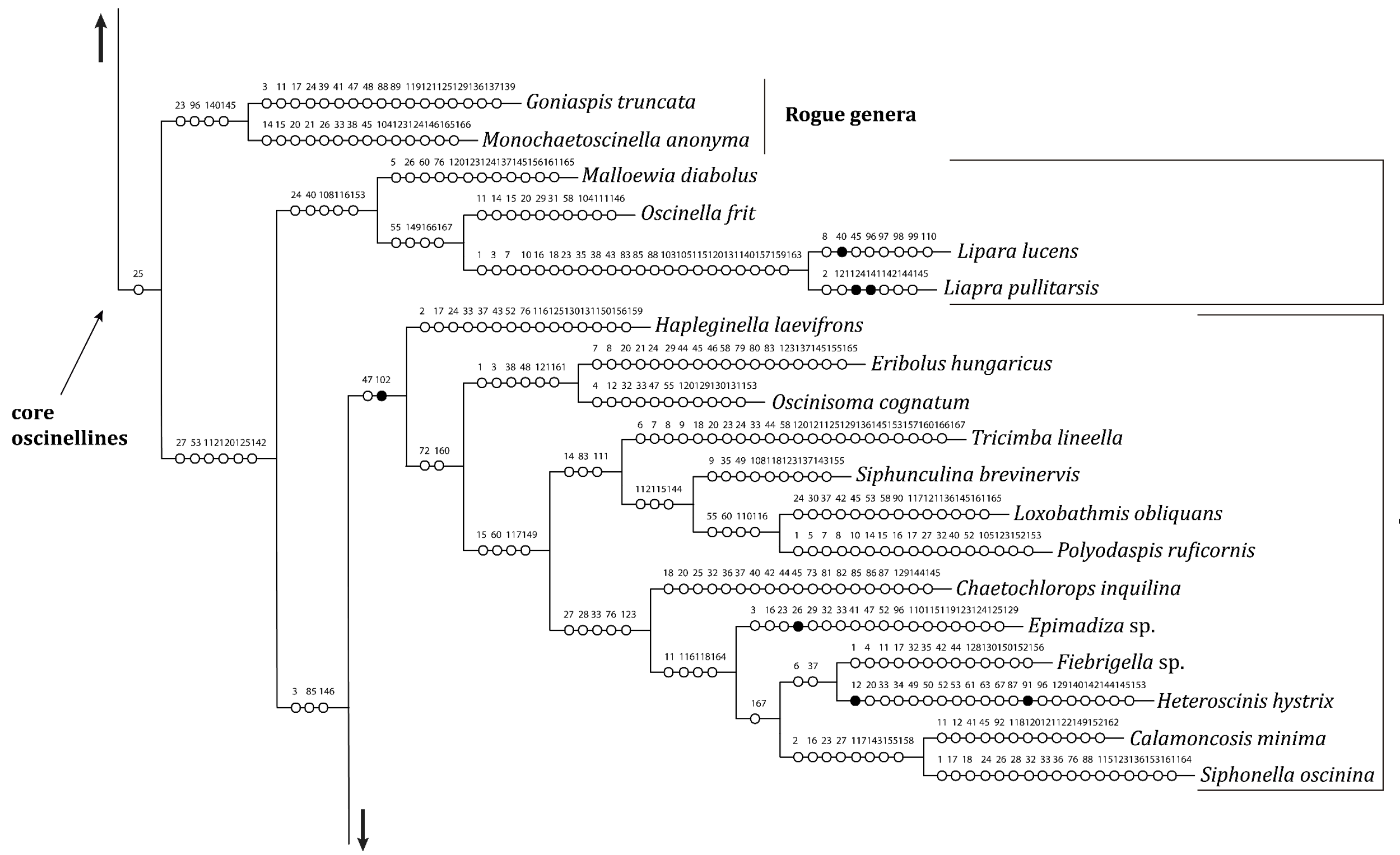




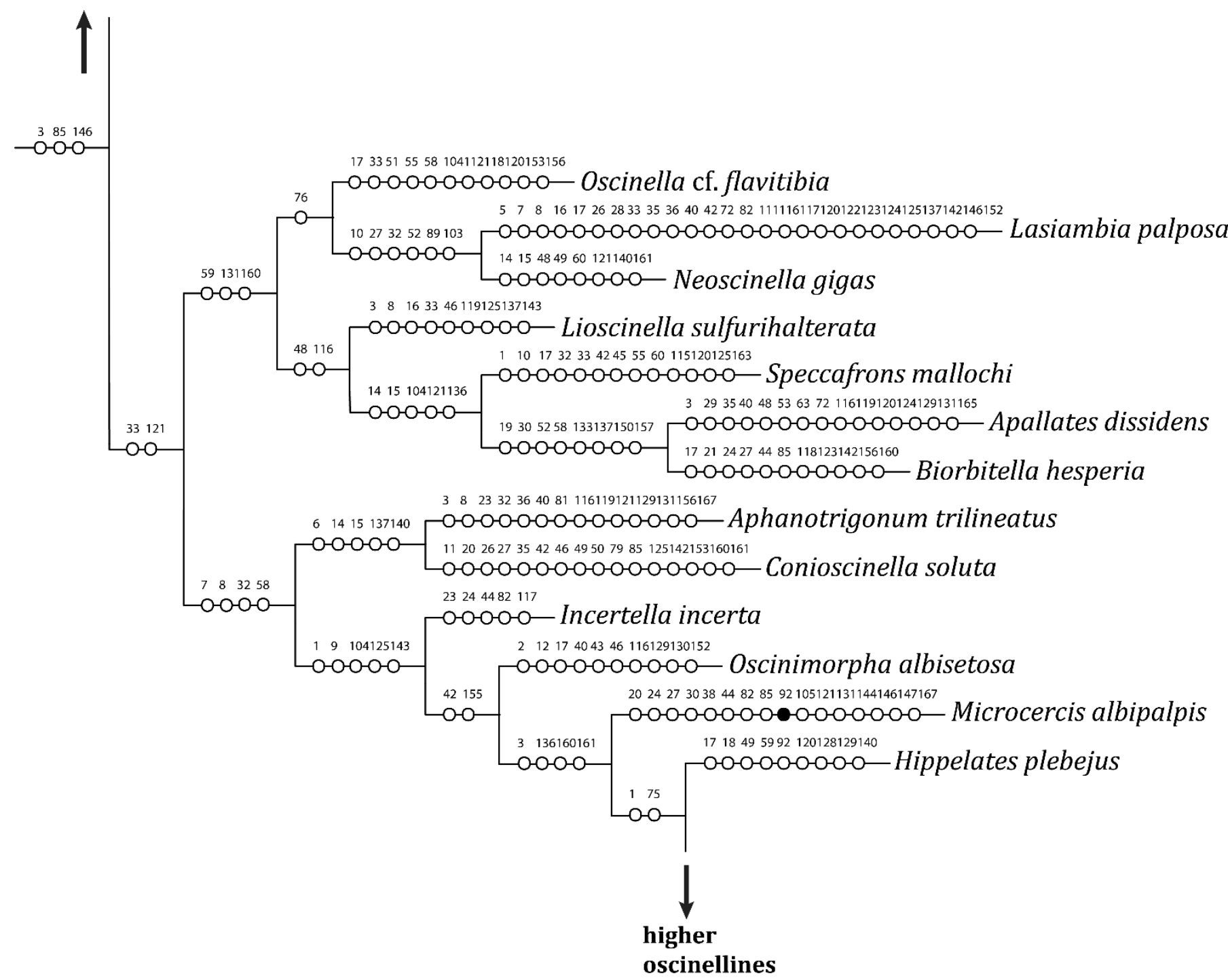

\section{Rogue genera}

Figure 7. The single maximum parsimonious tree in phylogeny, under implied weighting scheme, $\mathrm{k}=3, \mathrm{Ci}=0.14, \mathrm{Ri}=0.42$; with unambiguous mapped on branches. White circles, homoplastic synapomorphies; full black circles, non - homoplastic synapomorphies. (Continuing) 


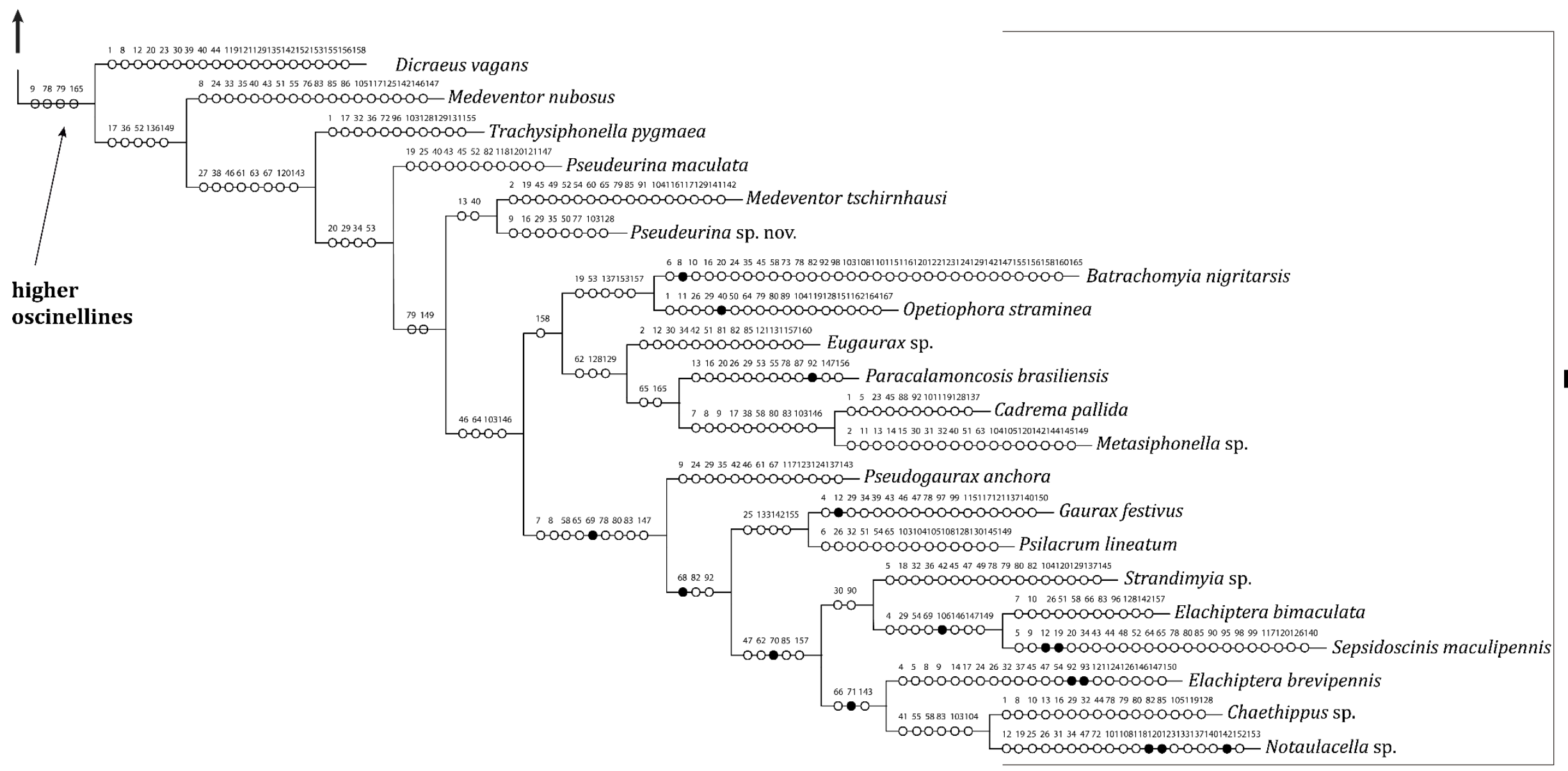

\section{Elachipterini}

Figure 8. The single maximum parsimonious tree in phylogeny, under implied weighting scheme, $\mathrm{k}=3, \mathrm{Ci}=0.14, \mathrm{Ri}=0.42 ;$ with unambiguous mapped on branches. White circles, homoplastic synapomorphies; full black circles, non - homoplastic synapomorphie. 


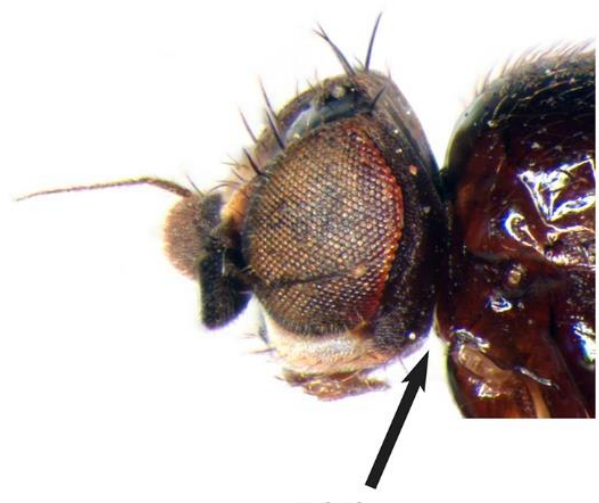

1(0)

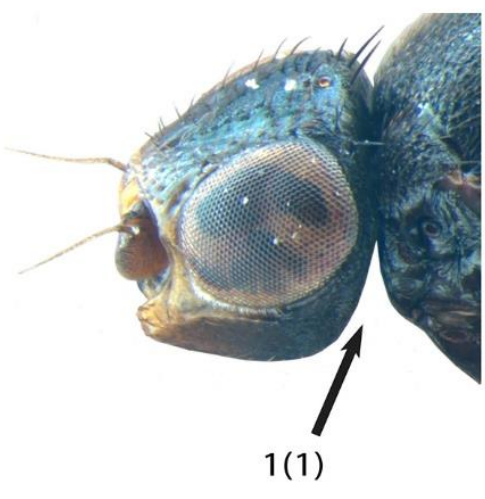

(b)

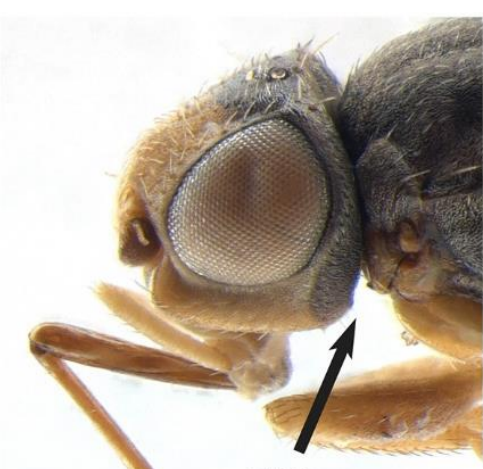

1(2)

(c)

Figure 9a-c. Character 1, connection of head capsule with thorax: a. Biorbitella Hesperia, state 0 , ventrally; b. Polyodaspis ruficornis, state 1, posteriorly; c. Oscinimorpha albisetosa, state 2, medially.

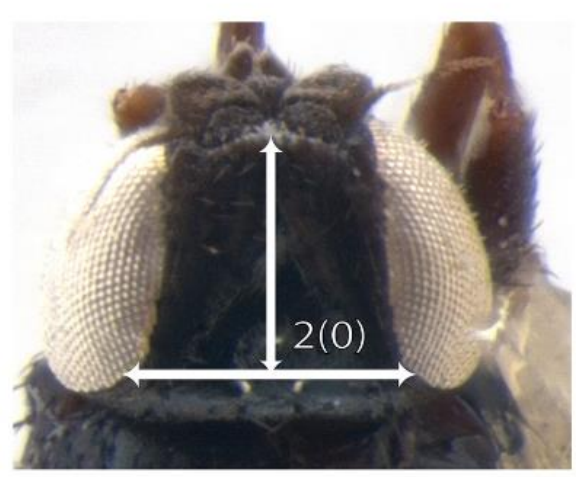

(a)

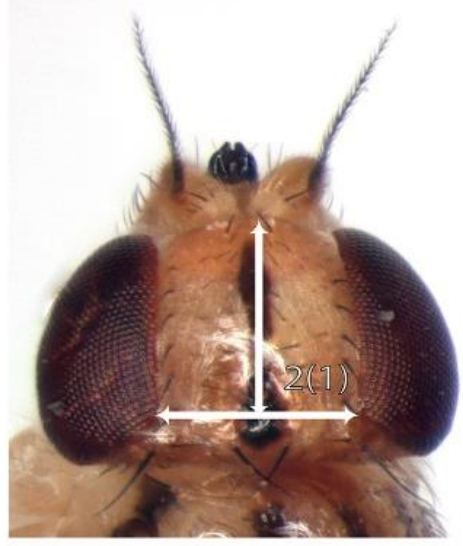

(b)

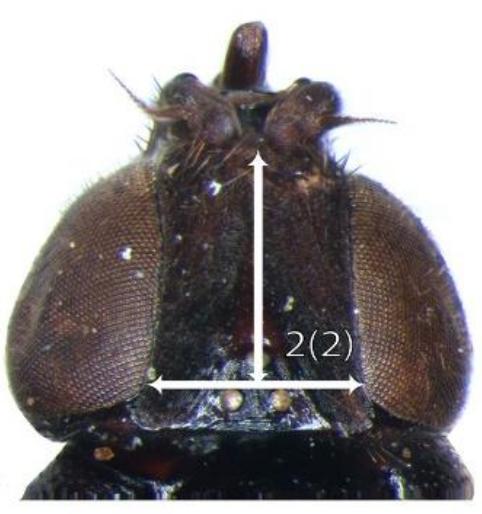

(c)

Figure 10a-c. Character 2, frons in dorsal view: a. Malloewia diabolus, state 0 , wider than long; $b$. Metasiphonella sp., state 1 , as wide as long; c. Siphonella oscinina, state 2, longer than wide. 


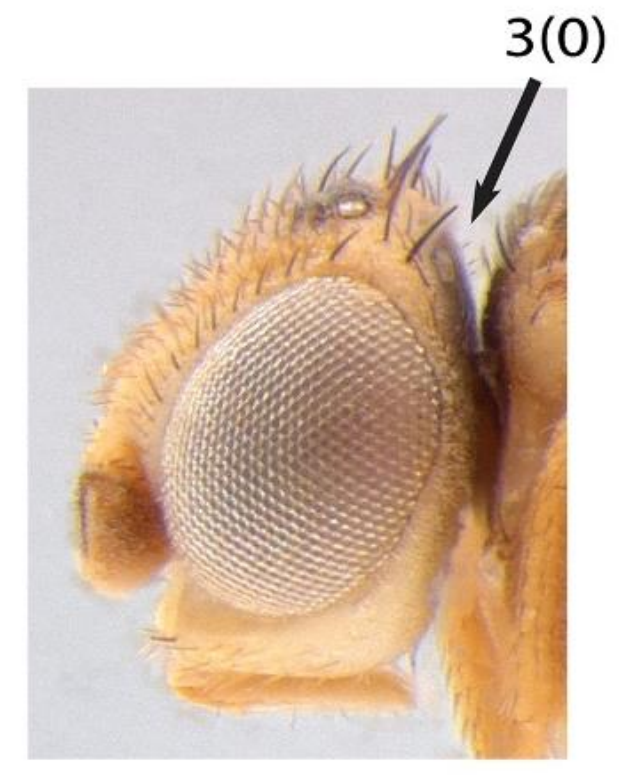

(a)

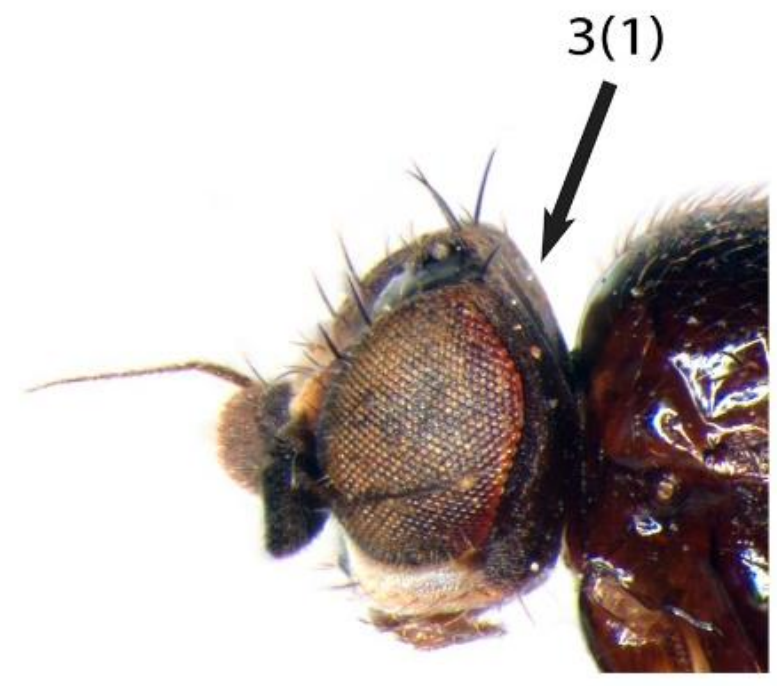

(b)

Figure 11a-b. Character 3, occiput: a. Trachysiphonella pygmaea, state 0, convex; b. Biorbitella hesperia, state 1, straight, angulated with frons.

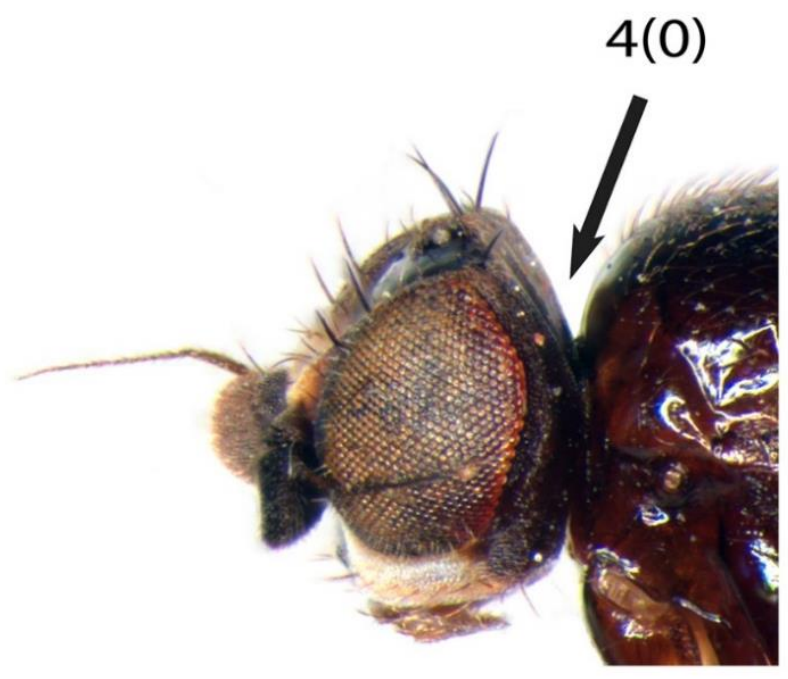

(a)

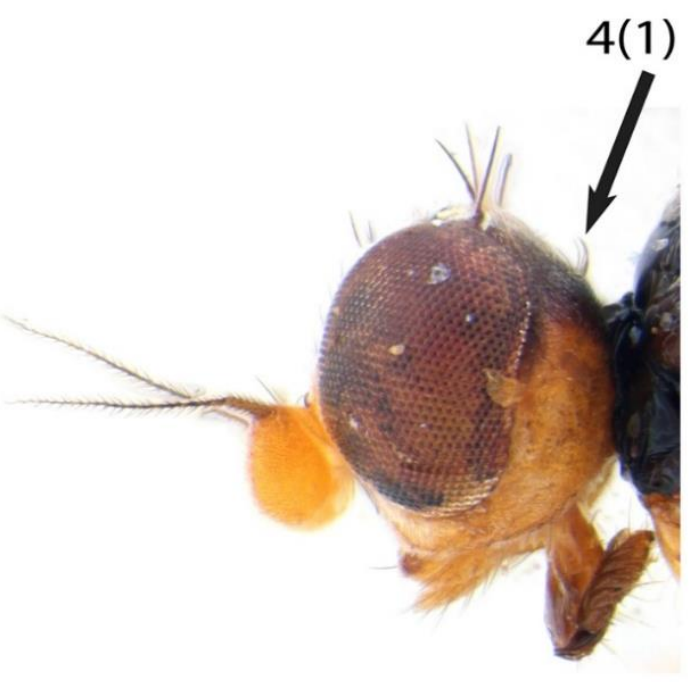

(b)

Figure 12a-b. Character 4, occiput with pair of setae, basally (supercervial setae): a. Biorbitella hesperia, state 0 , absent; b. Disciphus peregrinus, state 1 , present . 


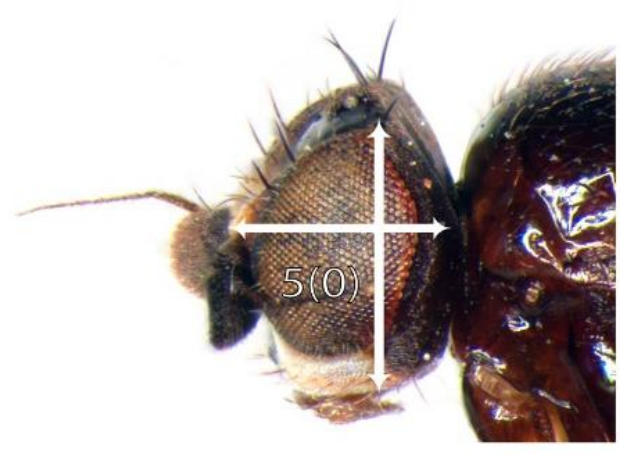

(a)

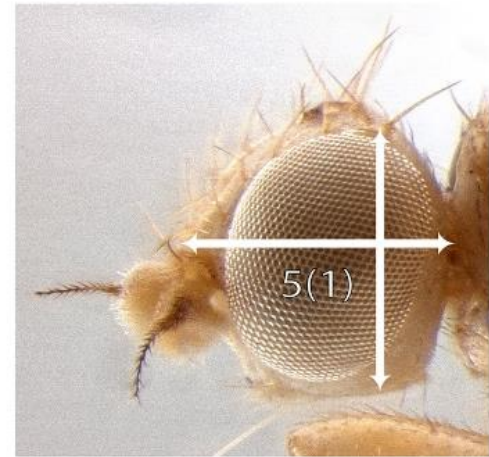

(b)

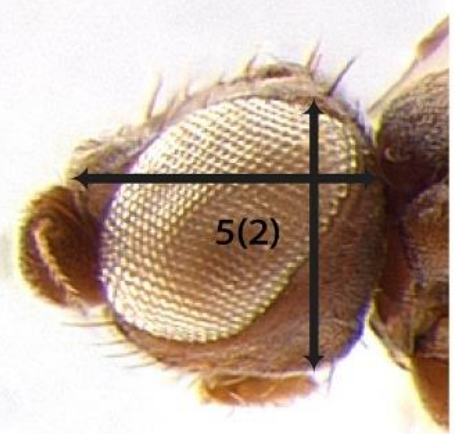

(c)

Figure 13a-c. Character 5, head in lateral view: a. Biorbitella hesperia, state 0 , longer than wide; $b$. Cadrema pallida, state 1, as wide as long; c. Eribolus hungaricus, state 2, wider than long.

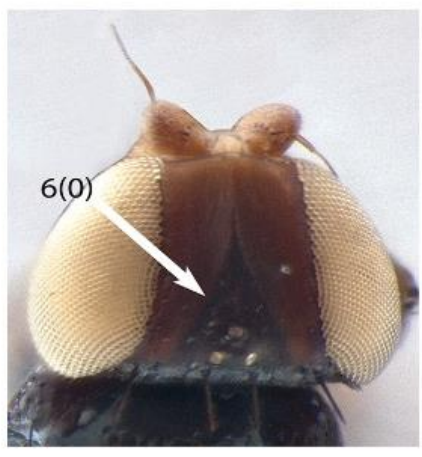

(a)

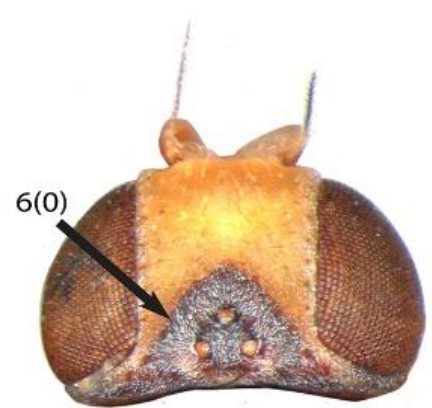

(b)

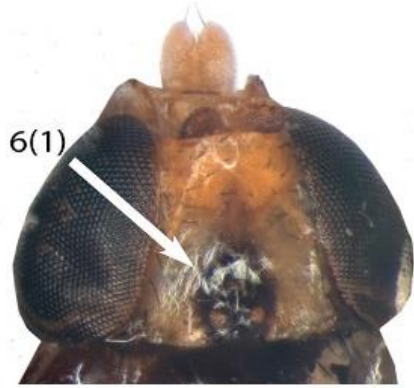

(c)

Figure 14a-c. Character 6, ocellar triangle: a. Epimadiza sp., state 0, well marked, margins of ocellar triangle conspicuous; b. Hippelates plebejus, state 0 ; c. Heteroscinis hystrix, state 1 , not well marked, margins not clear or entirely absent. 


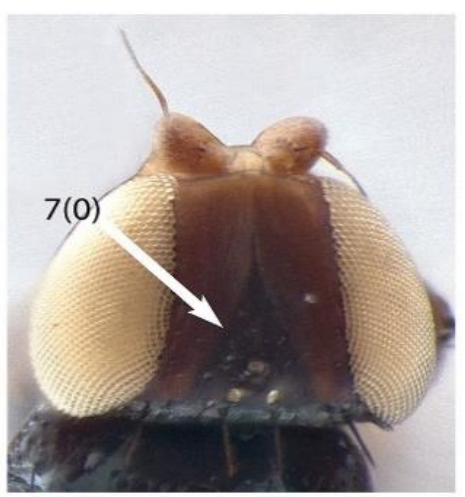

(a)

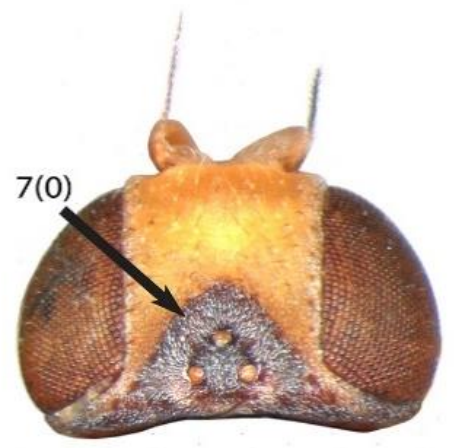

(b)

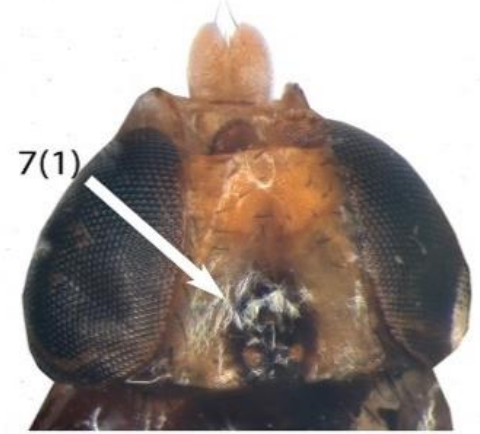

(c)

Figure 15a-c. Character 7, texture of ocellar triangle: a. Epimadiza sp., state 0, opaque, rugose; b. Hippelates plebejus, state 0 ; c. Heteroscinis hystrix, state 1, shinning.

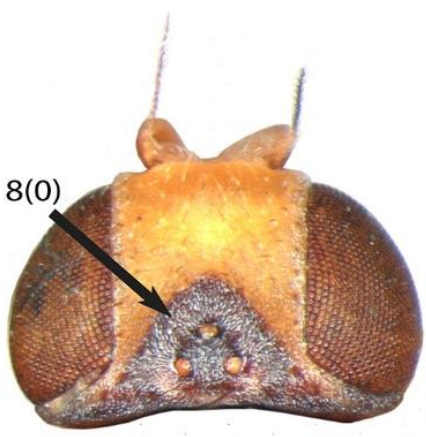

(a)

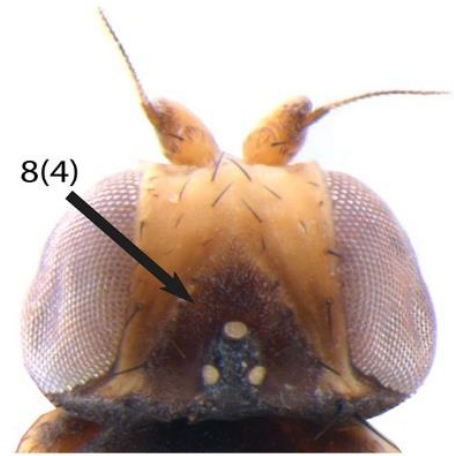

(d)

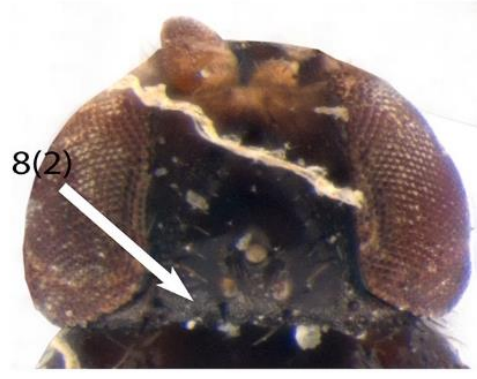

(b)

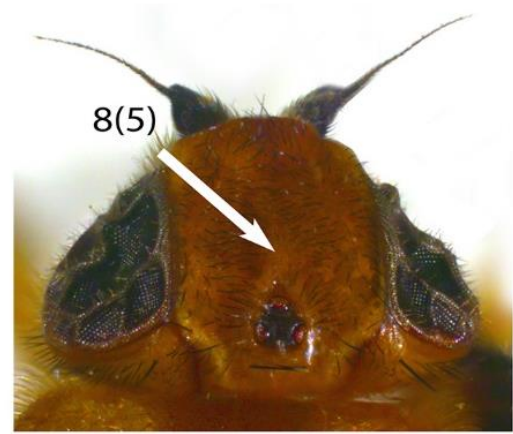

(e)

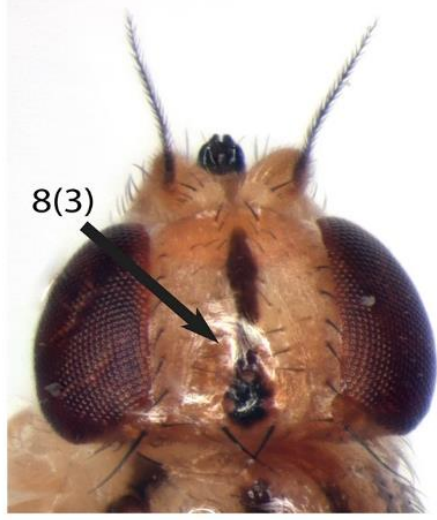

(c)

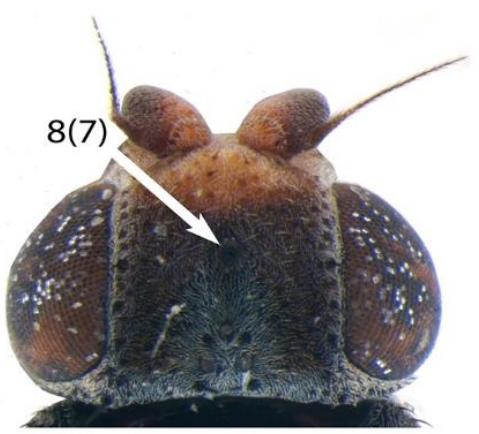

(f)

Figure 16a-f. Character 8 , pruinosity of ocellar triangle without tubercle: a. Hippelates plebejus, state 0 , almost entirely pubescent; $b$. Lioscinella sulfurihalterata, state 2 , posterior third pubescent; c. Metasiphonella sp., state 3, almost bare; d. Dicraeus vagans, state 4, anterior two third pubescent; e. Batrachomyia nigritarsis, state 5, anterior third pubescent; f. Aphanotrigonum trilineatus, state 7, anterior tip of triangle pubescent. 


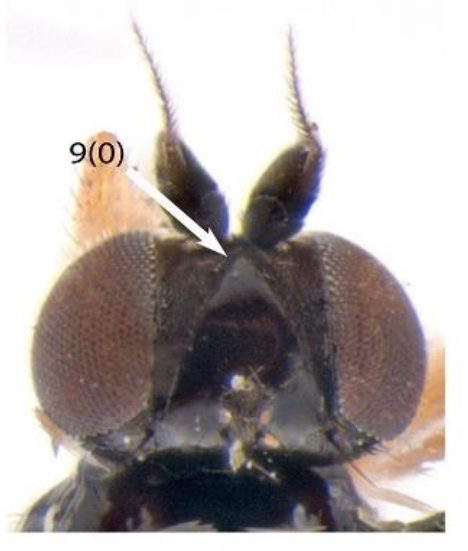

(a)

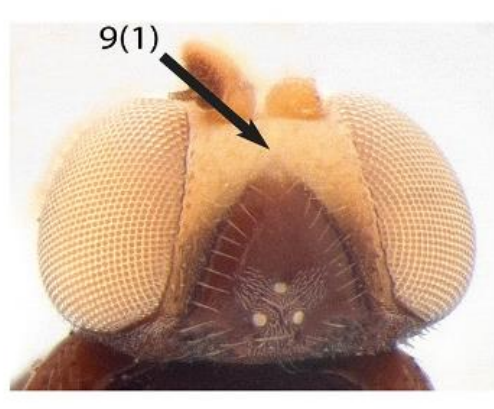

(b)

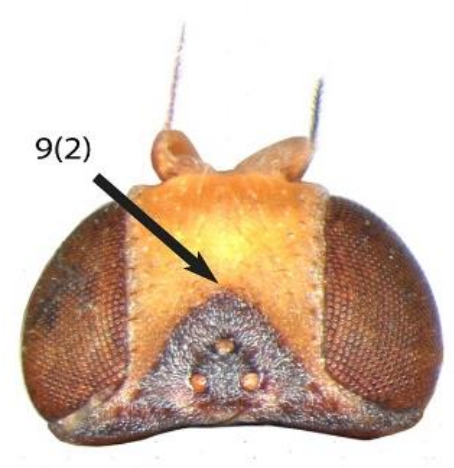

(c)

Figure 17a-c. Character 9, anterior end of ocellar triangle: a. Monochaetoscinella anonyma, state 0 , reaching or almost reaching anterior margin of frons; b. Oscinisoma cognatum, state 1 , between anterior margin of frons and middle of frons; c. Hippelates plebejus, state 2, at most reaches middle of frons.

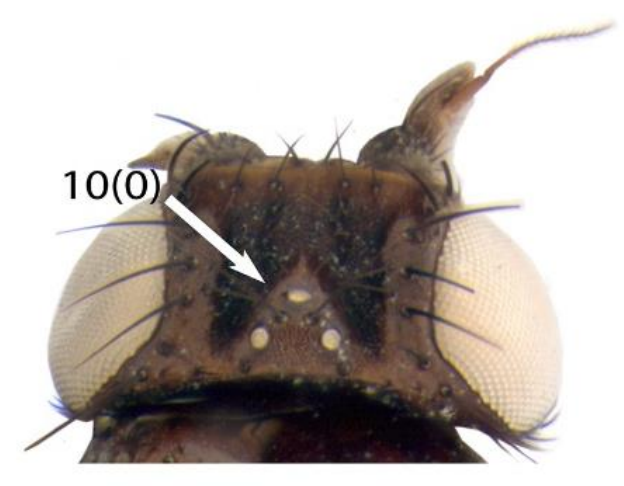

(a)

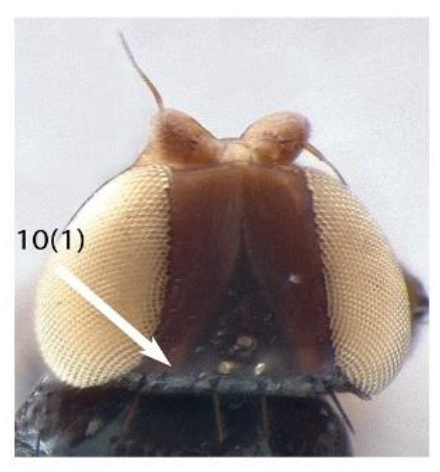

(b)

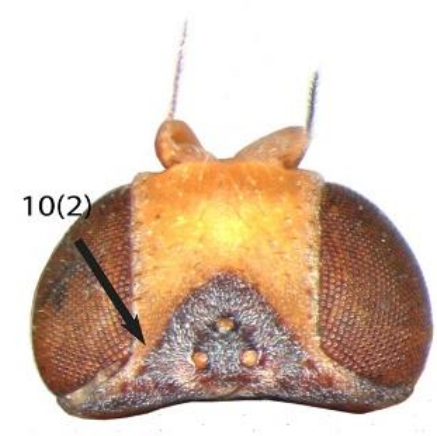

(c)

Figure 18a-c. Character 10, ocellar triangle width at posterior margin: a. Phyllomyza flavitarsis, state 0 , hardly wider than the tubercle itself; b. Epimadiza sp., state 1, wider than tubercle, reaching halfway to eye margin; c. Hippelates plebejus, state 2, getting close or laterally reaching the eye margins. 


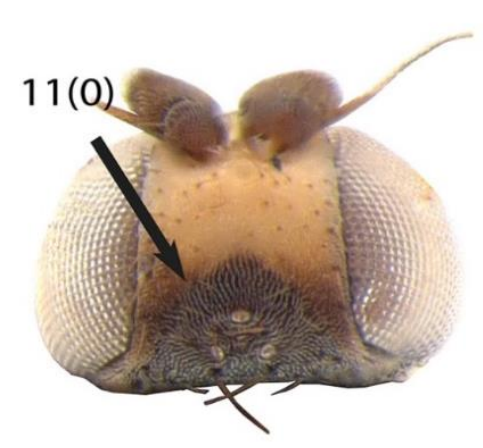

(a)

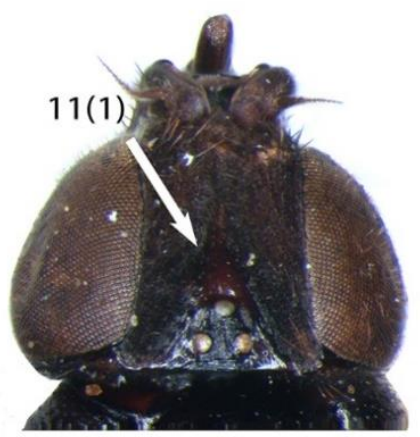

(b)

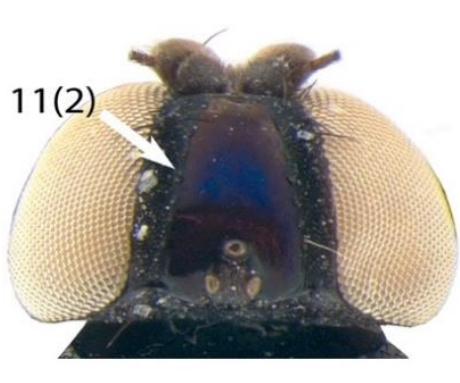

(c)

Figure 19a-c. Character 11, lateral margins of ocellar triangle: a. Microcercis albipalpis, state 0 , convex; b. Siphonella oscinina, state 1 , concave; c. Rhodesiella sp.2, state 2 , straight. 


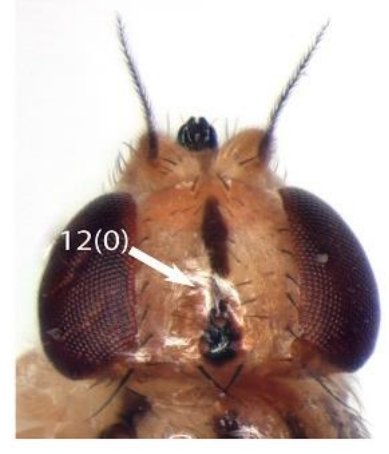

(a)

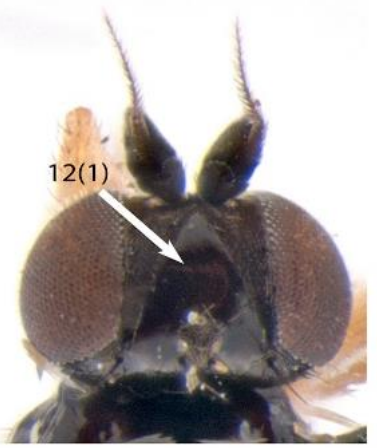

(d)

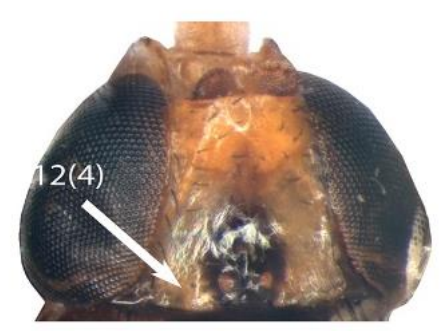

(g)

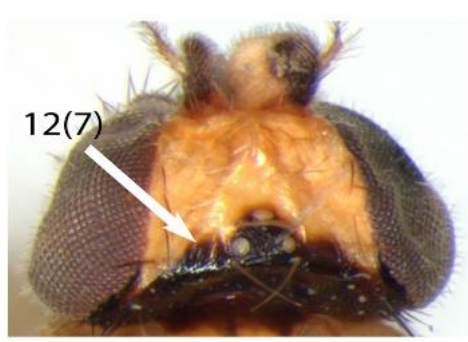

(j)

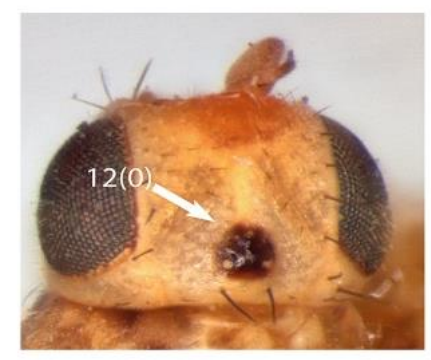

(b)

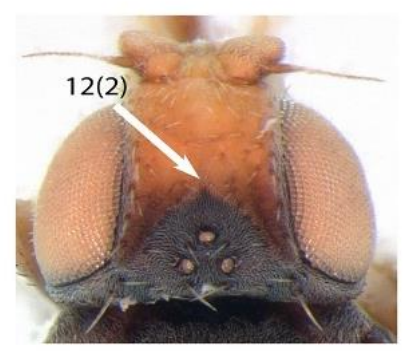

(e)

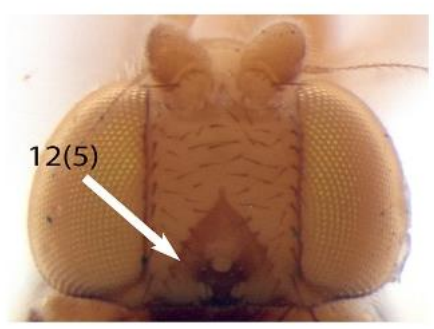

(h)

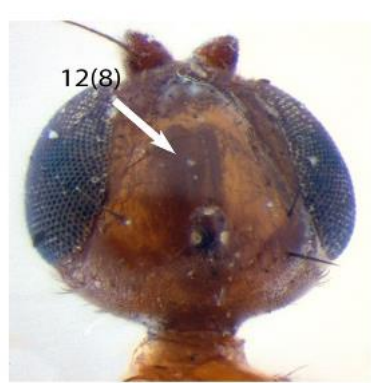

(k)

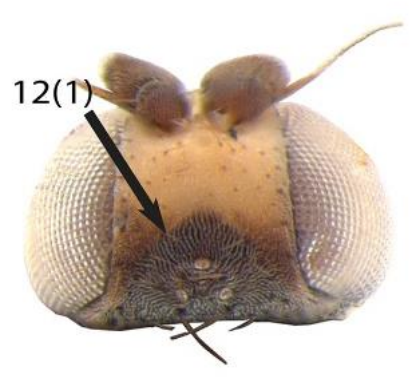

(c)

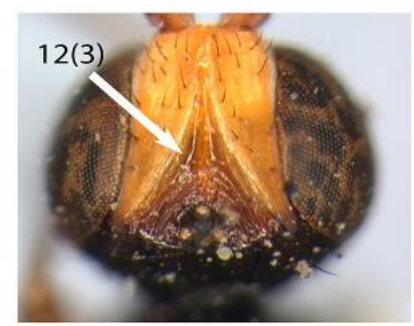

(f)

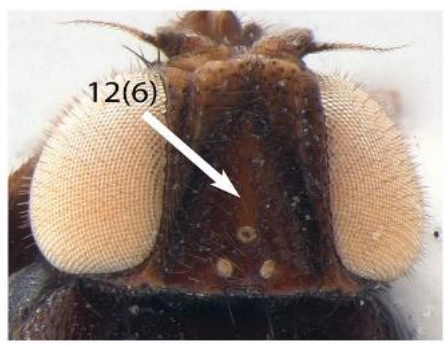

(i)

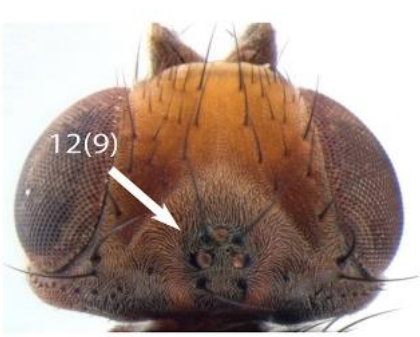

(I)

Figure 20a-I. Character 12, color of ocellar triangle: a. Metasiphonella sp., state 0 , light brown to yellow entirely; b. Opetiophora straminea, state 0; c. Microcercis albipalpis, state 1, dark brown to black entirely; d. Monochaetoscinella anonyma, state 1; e. Oscinimorpha albisetosa, state 2, anterior end light, the rest dark; f. Sagareocerus aberrans, state 3, anterior half light, posterior half dark; g. Heteroscinis hystrix, state 4, posterior end light, the rest dark; h. Eugaurax sp., state 5, posterior light, anterior dark; i. Calamoncosis minima, state 6, middle (oval or linear) light, around dark; j. Gaurax festivus, state 7, posterior end dark, the rest light; k. Sepsidoscinis maculipennis, state 8 , anterior fifth and a median band dark, with dark yellow background; I. Siphonellopsis lacteibasis, state 9, dark tubercle with darker lateral areas over lighter background. 


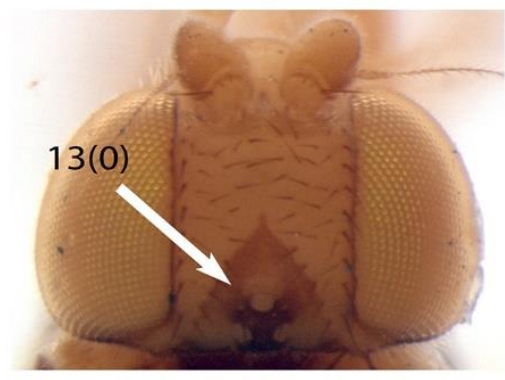

(a)

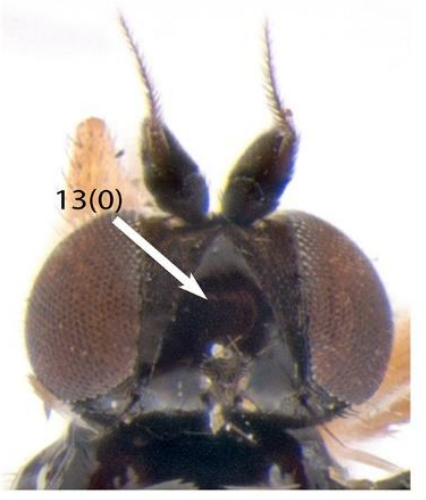

(b)

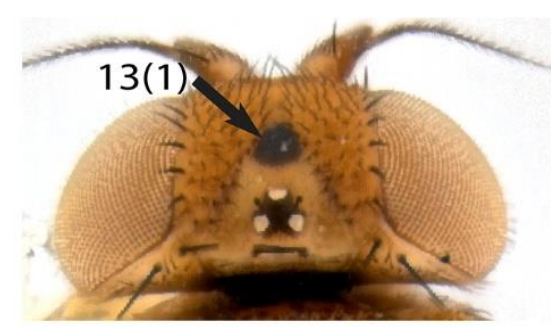

(c)

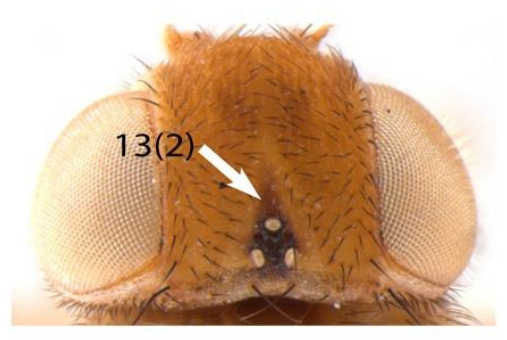

(d)

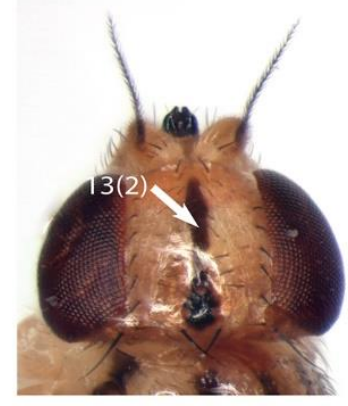

(e)

Figure 21a-e. Character 13, ocellar triangle: a. Eugaurax sp., state 0 , concolor; b.

Monochaetoscinella anonyma, state 0; c. Chaethippus sp., state 1, with spot; d. Medeventor tschirnhausi, state 2, with band; e. Metasiphonella sp., state 2.

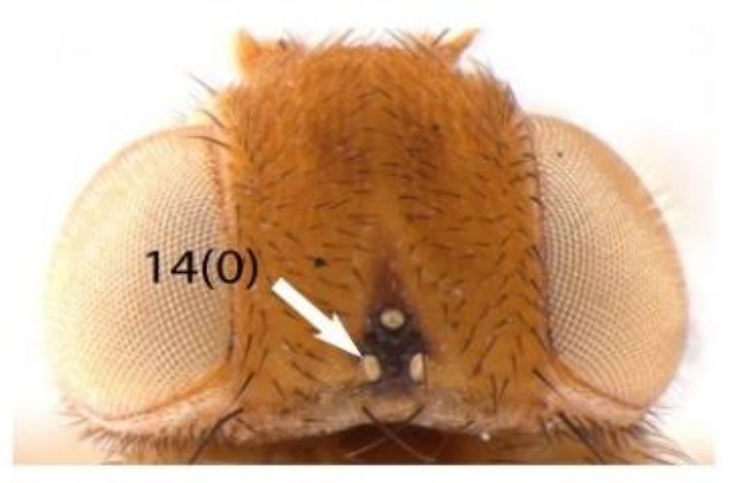

(a)

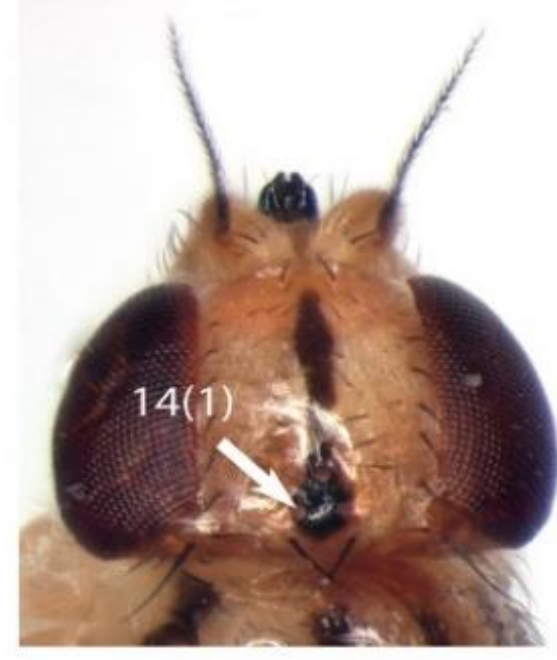

(b)

Figure 22a-b. Character 14, posterior ocelli: a. Medeventor tschirnhausi, state 0 , yellow or light brown; b. Metasiphonella sp., state 1, black or dark brown. 


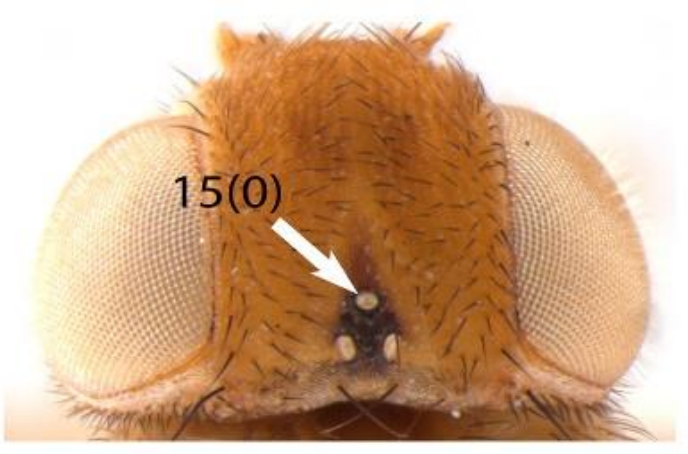

(a)

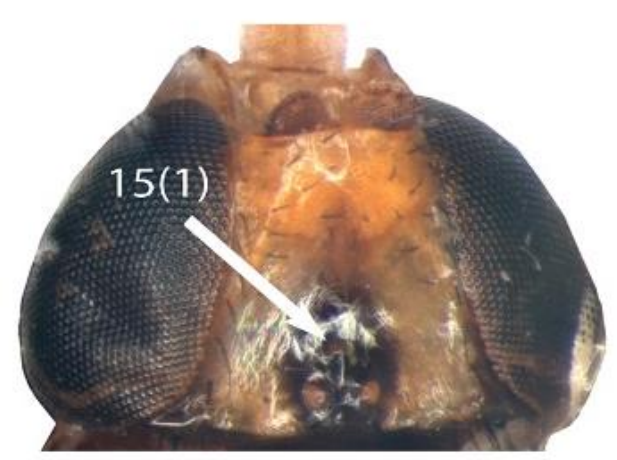

(b)

Figure 23a-b. Character 15, anterior ocellus: a. Medeventor tschirnhausi, state 0 , yellow or light brown; b. Heteroscinis hystrix, state 1 , black or dark brown.

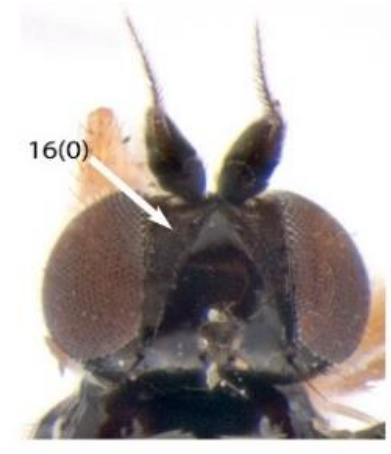

(a)

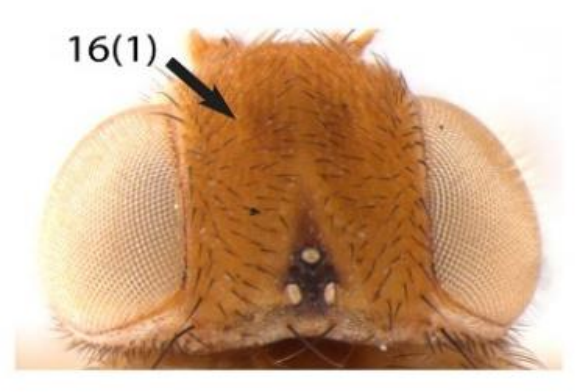

(b)

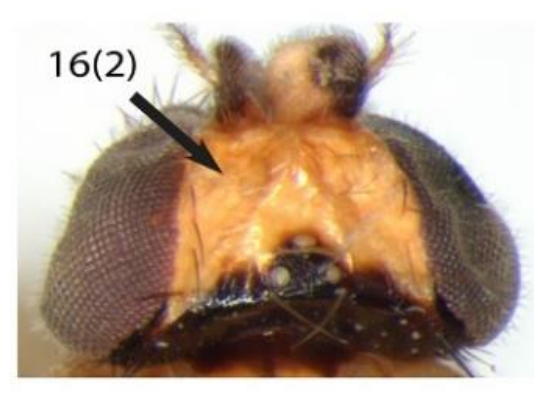

(c)

Figure 24a-c. Character 16, density of setation on frons: a. Monochaetoscinella anonyma, state 0 , bare; b. Medeventor tschirnhausi, state 1, dense; c. Gaurax festivus, state 2, scattered. 


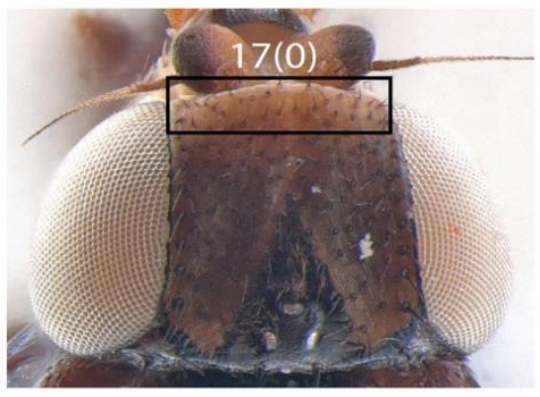

(a)

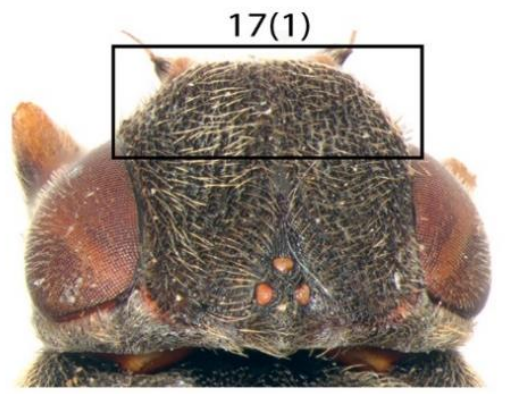

(b)

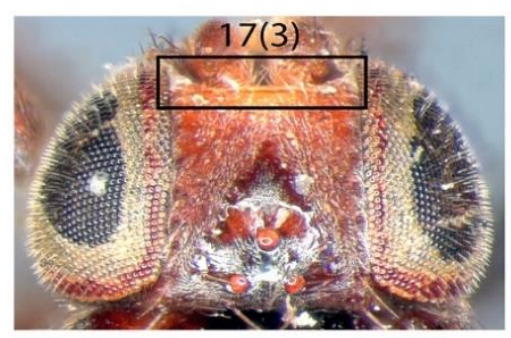

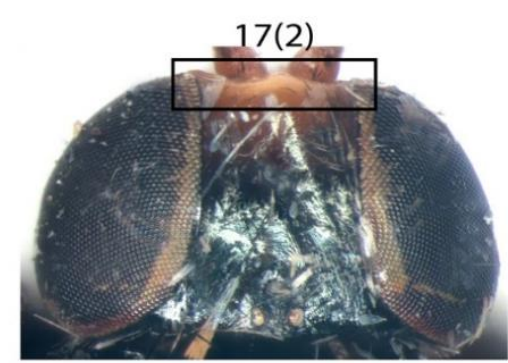

(c)

\section{(d)}

Figure 25a-d. Character 17, anterior margin of frons in dorsal view (in comparison of the level of eyes): a. Lasiambia palposa, state 0 , straight or slightly projected; b. Lipara lucens, state 1 , projected moderately beyond the anterior margin of eyes; c. Fiebrigella sp., state 2 , concave, medially with projection; d. Speccafrons mallochi, state 3 , concave, medially straight.

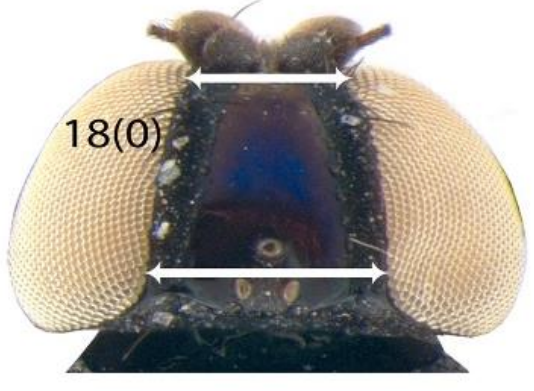

(a)

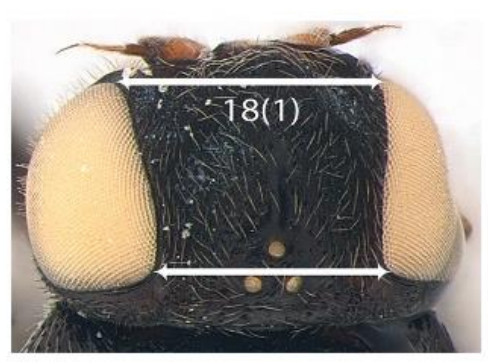

(b)

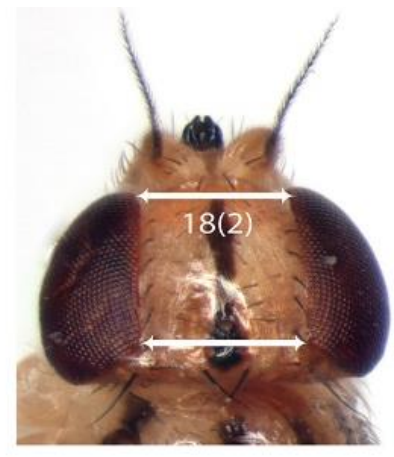

(c)

Figure 26a-c. Character 18, lateral margins of frons in dorsal view: a. Rhodesiella sp.2, state 0 , converging towards anterior margin; b. Lipara pullitarsis, state 1 , diverging towards anterior margin; c. Metasiphonella sp., state 2, almost parallel. 


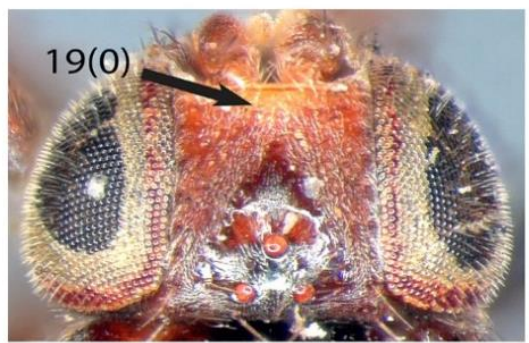

(a)

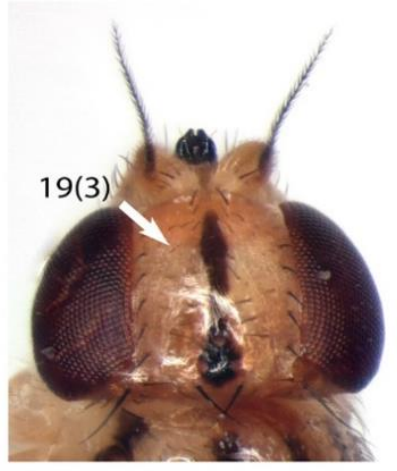

(d)

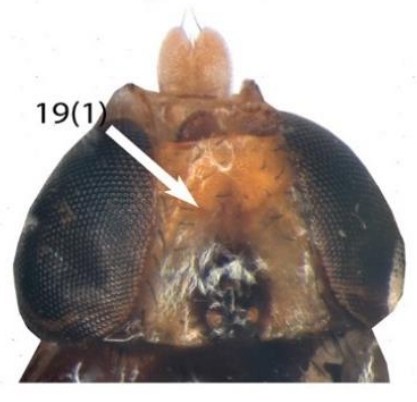

(b)

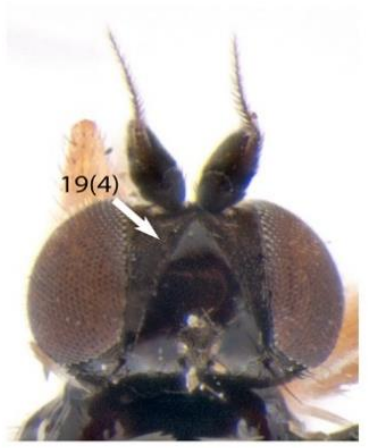

(e)

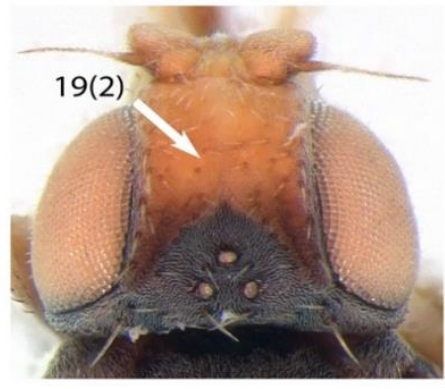

(c)

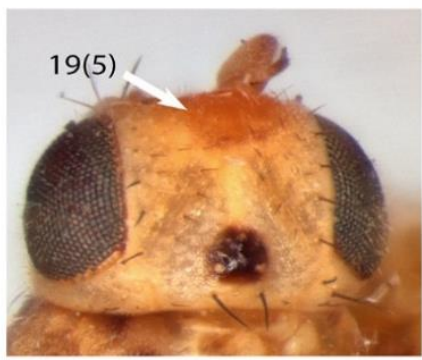

(f)

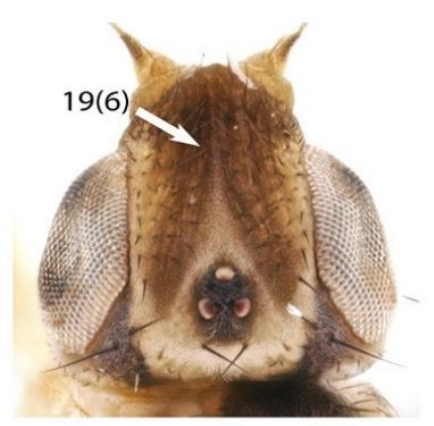

(g)

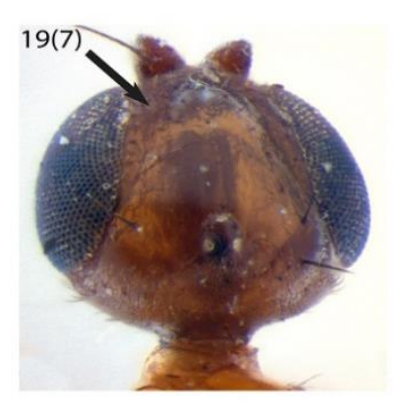

(h)

Figure 27a-h. Character 19, ground color of frons: a. Speccafrons mallochi, state 0 , anterior third yellow; b. Heteroscinis hystrix, state 1, anterior half yellow; c. Oscinimorpha albisetosa, state 2, anterior two third yellow; d. Metasiphonella sp., state 3, entirely yellow; e. Monochaetoscinella anonyma, state 4, entirely dark; f. Opetiophora straminea, state 5 , anterior third dark; $g$. Pseudeurina maculata (photography by Barbara Ismay), state 6, anterior two third dark; $h$. Sepsidoscinis maculipennis, state 7, anterior half dark. 


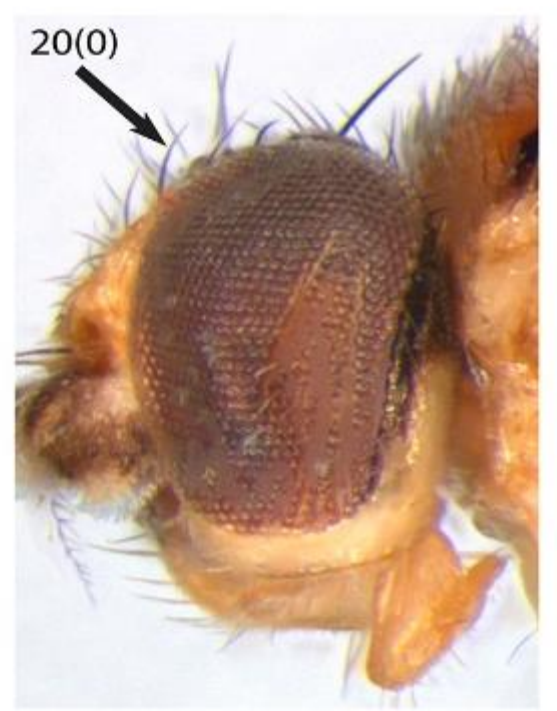

(a)

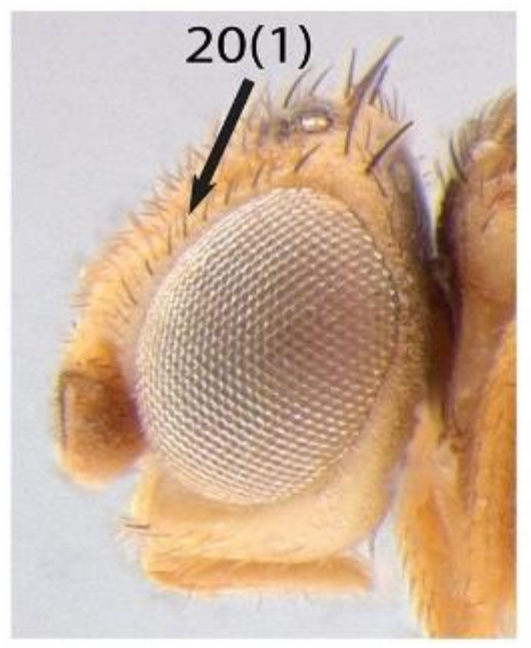

(b)

Figure 28a-b. Character 20, length of orbital setae: a. Gaurax festivus, state 0, long; b. Trachysiphonella pygmaea, state 1 , short.

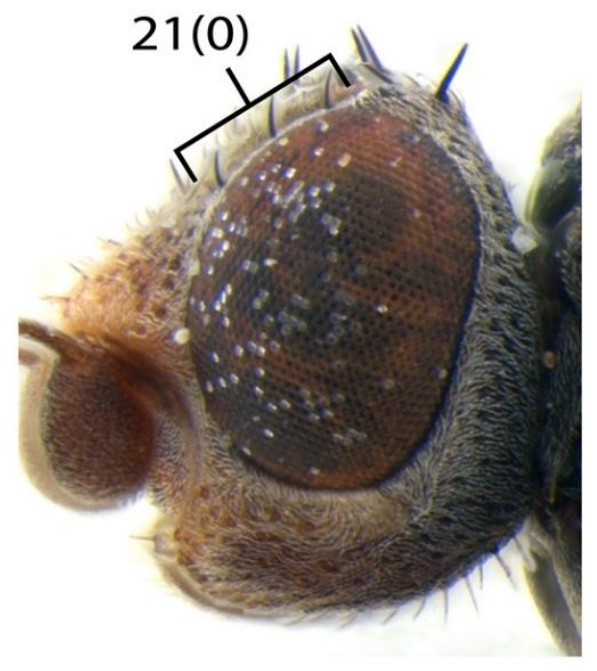

(a)

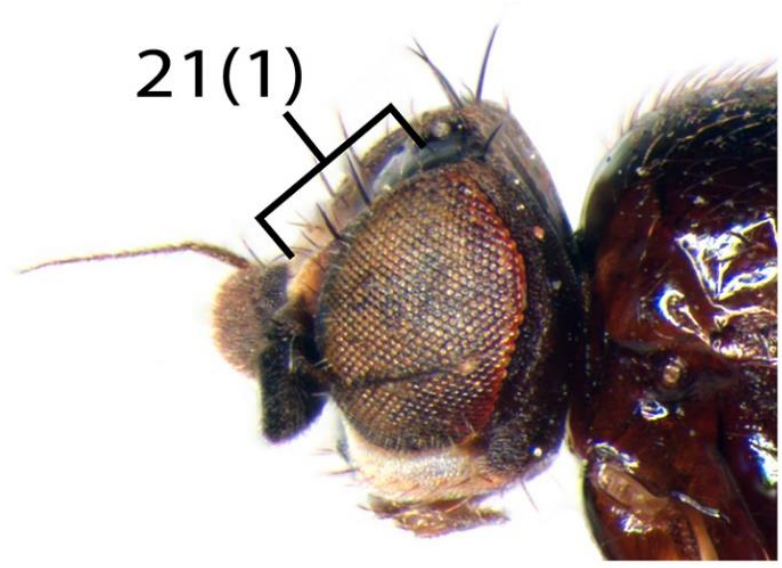

(b)

Figure 29a-b. Character 21, comparison of length of orbital setae to each other: a. Aphanotrigonum trilineatus, state 0 , of same length; b. Biorbitella Hesperia, state 1, some clearly longer than others. 


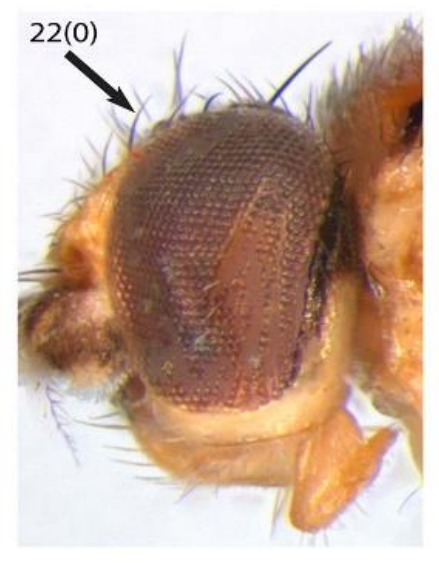

(a)

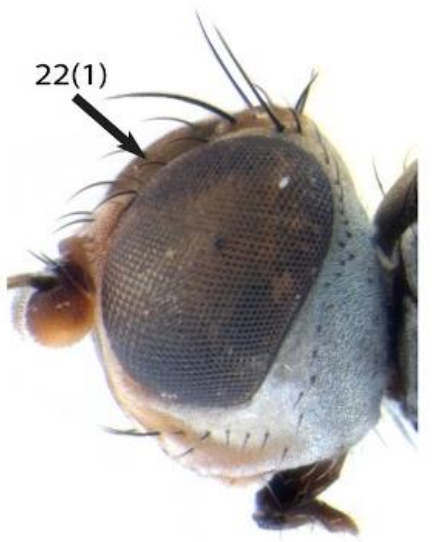

(b)

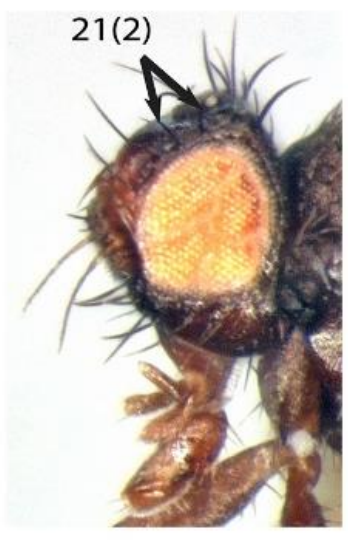

(c)

Figure 30a-c. Character 22, direction of orbital setae: a. Gaurax festivus, state 0 , all reclinate; $b$. Siphonellopsis lacteibasis, state 1 , all proclinate; c. Meoneura flavifacies, state 2, anterior one proclinate, posterior two reclinate.

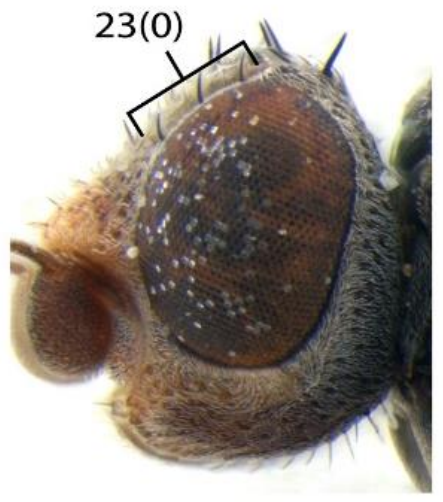

(a)

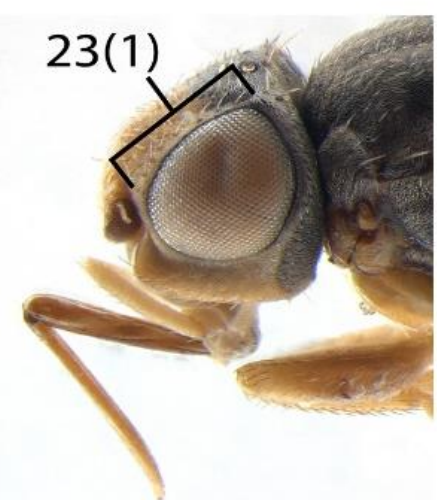

(b)

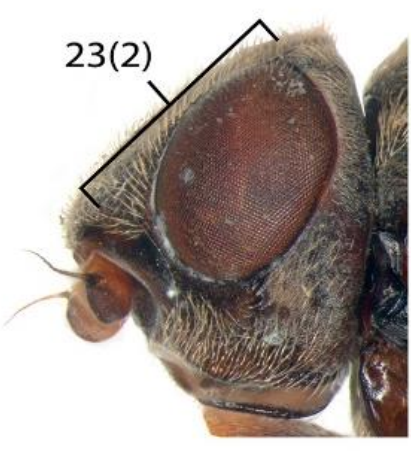

(c)

Figure 31a-c. Character 23, number of orbital setae: a. Aphanotrigonum trilineatus, state 0 , less than five; b. Oscinimorpha albisetosa, state 1, between five and ten; c. Lipara lucens, state 2, more than ten. 


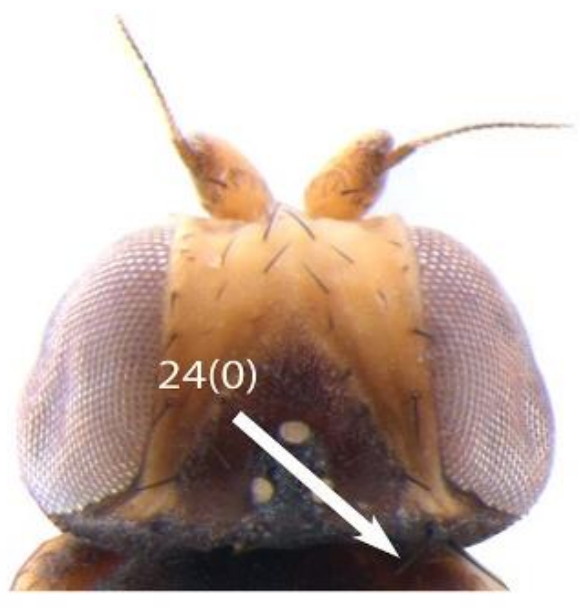

(a)

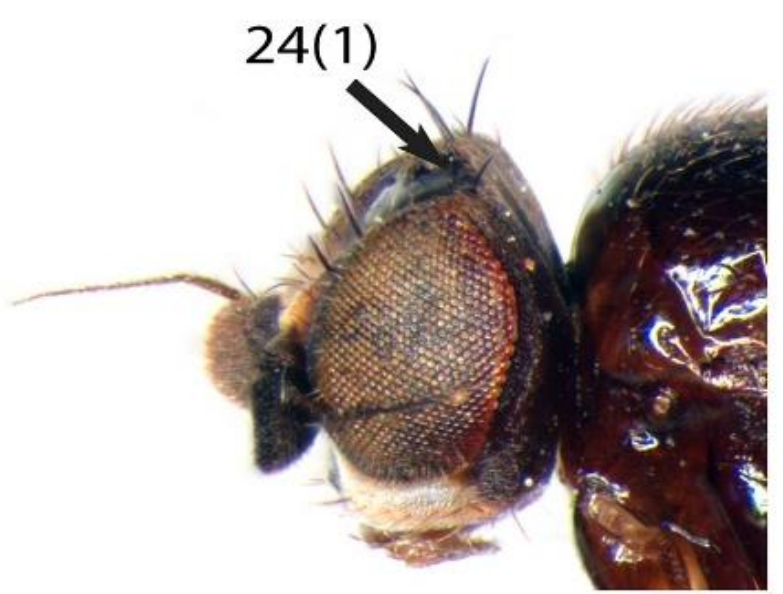

(b)

Figure 32a-b. Character 24, inner vertical seta: a. Dicraeus vagans, state 0 , conspicuous and strong; b. Biorbitella hesperia, state 1 , absent or not distinguishable.

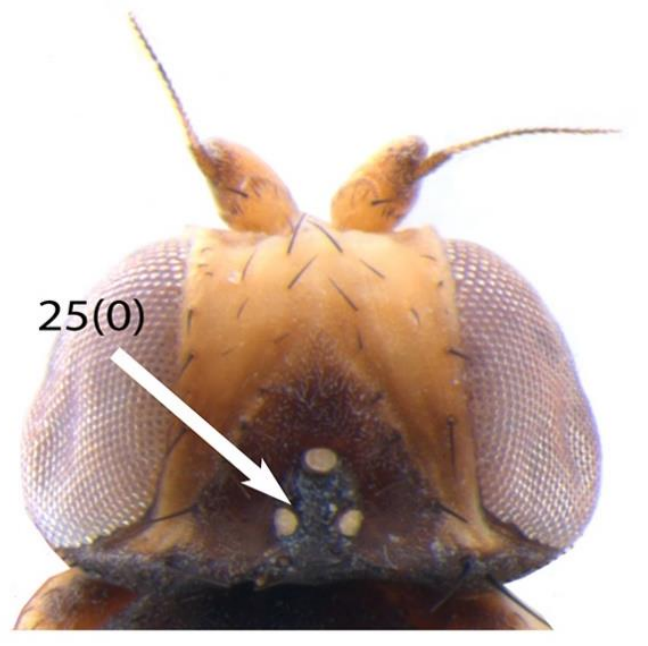

(a)

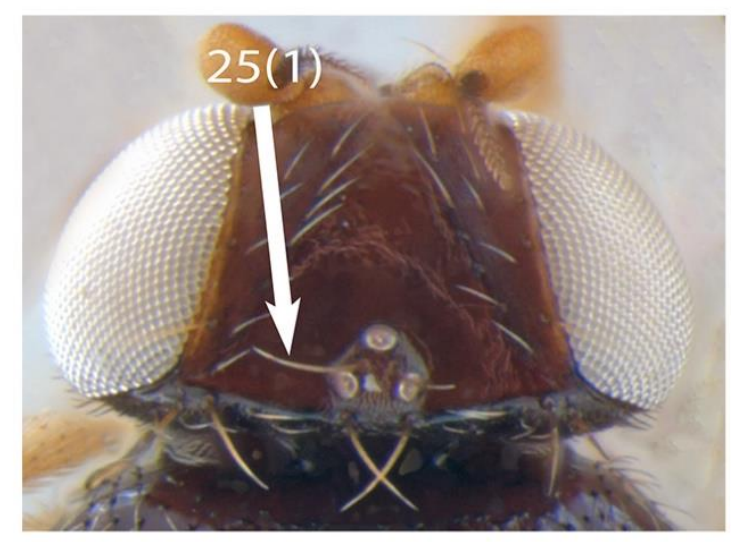

(b)

Figure 33a-b. Character 25, length of ocellar setae: a. Dicraeus vagans, state 0 , shorter than or as long as ocellar tubercle; b. Rhodesiella sp.1, state 1, longer than ocellar tubercle. 


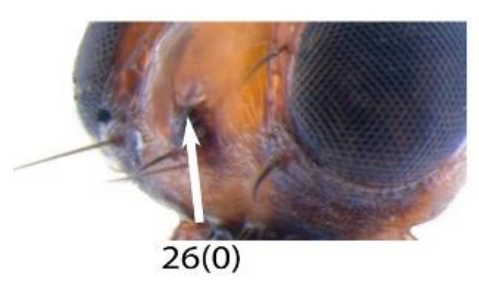

(a)

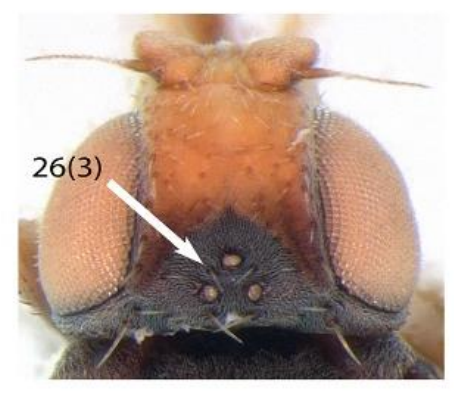

(d)

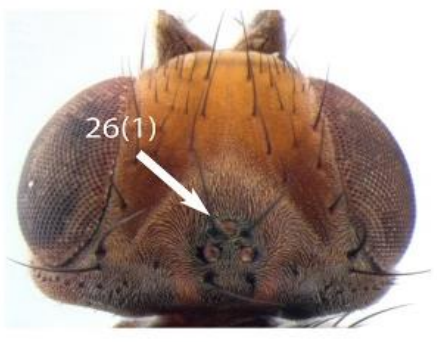

(b)

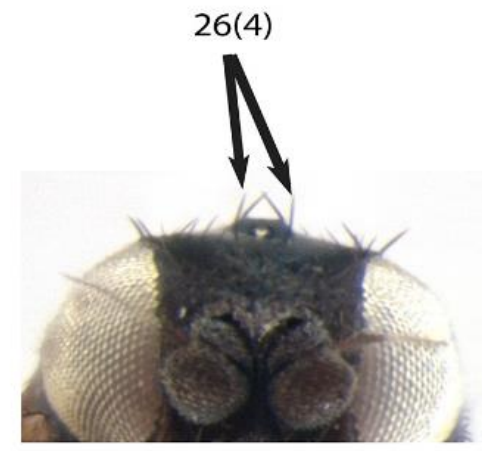

(e)

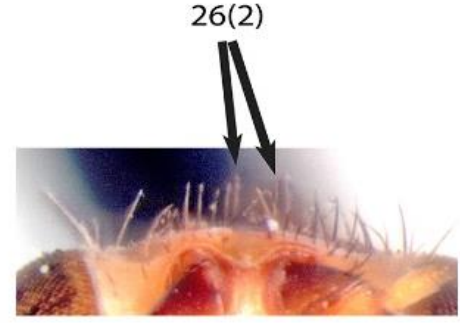

(c)

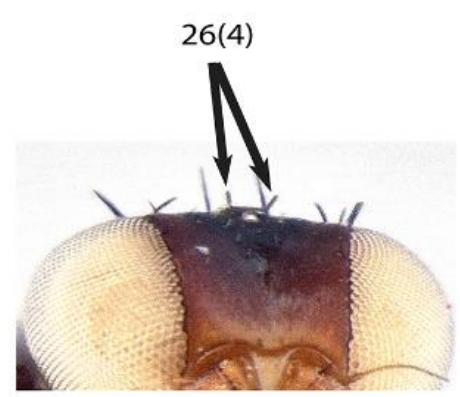

(f)

Figure 34a-f. Character 26, direction and orientation of ocellar setae: a. Elachiptera brevipennis, state 0 , proclinate and convergent; b. Siphonellopsis lacteibasis, state 1, proclinate and divergent; c. Diplotoxa versicolor, state 2 , proclinate and parallel; d. Oscinimorpha albisetosa, state 3 , reclinate and convergent; e. Malloewia diabolus, state 4, reclinate and parallel; f. Epimadiza sp., state 4.

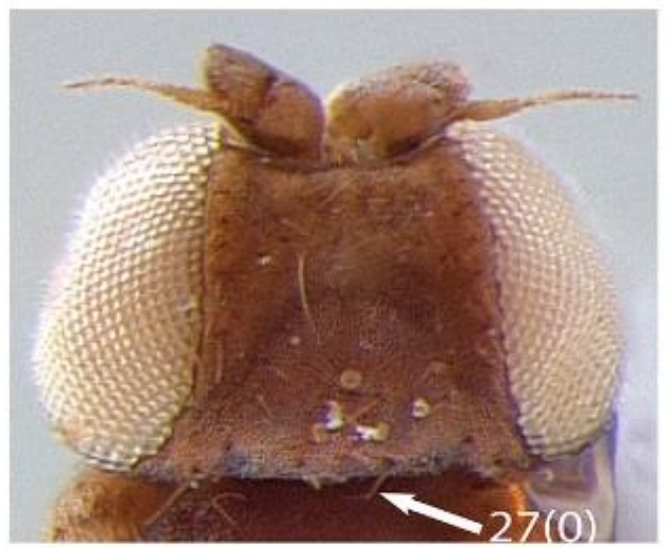

(a)

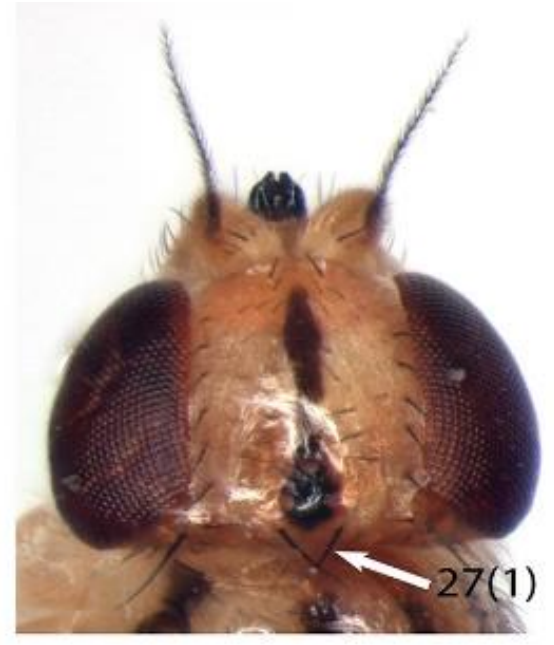

(b)

Figure 35a-b. Character 27, length of post-ocellar setae: a. Eribolus hungaricus, state 0 , shorter or as long as the ocellar tubercle; b. Metasiphonella sp., state 1 , longer than the ocellar tubercle. 


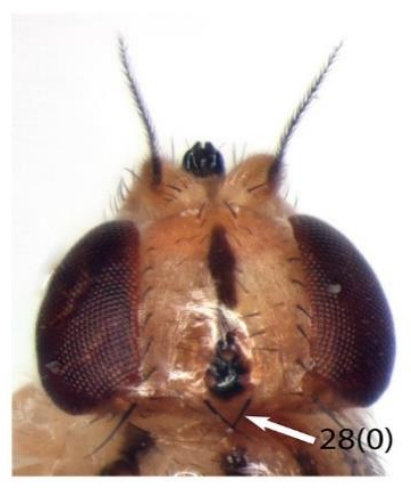

(a)

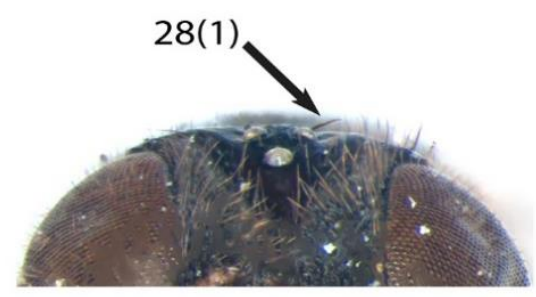

(b)

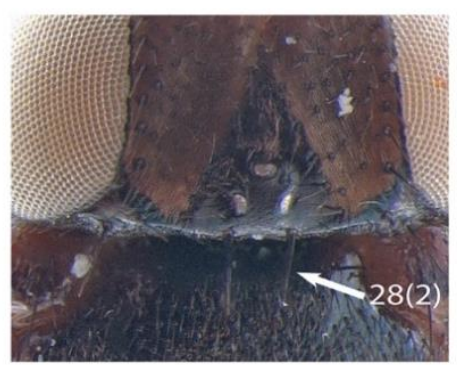

(c)

Figure 36a-c. Character 28, orientation of postocellar setae: a. Metasiphonella sp., state 0 , convergent; b. Siphonella oscinina, state 1, divergent; c. Lasiambia palposa, state 2, parallel.

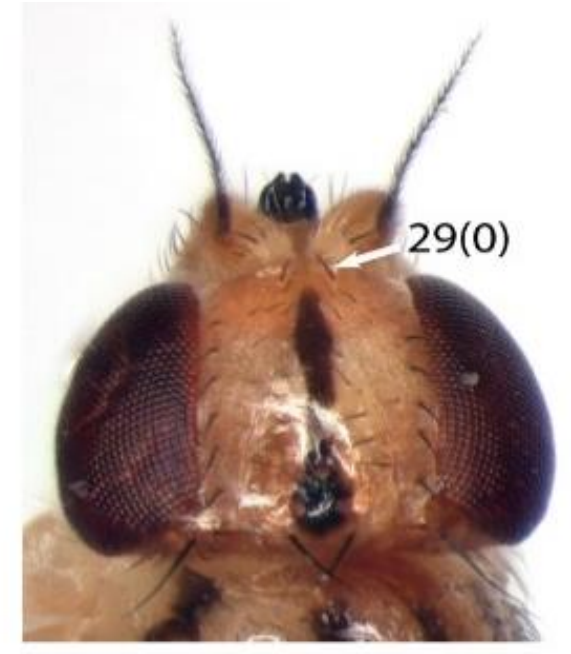

(a)

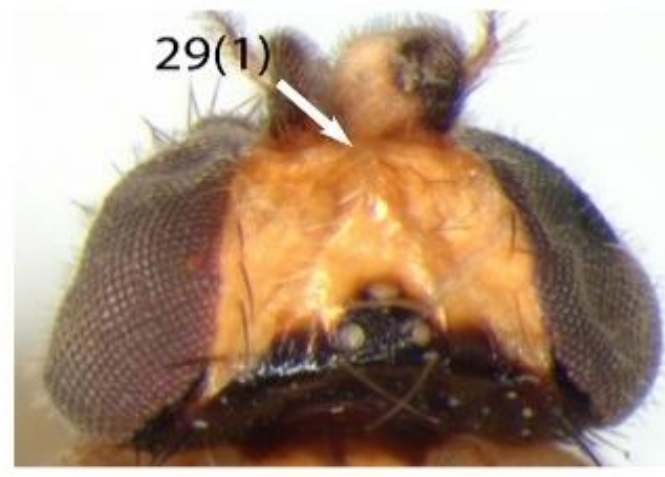

(b)

Figure 37a-b. Character 29, anterior interfrontal setae: a. Metasiphonella sp., state 0 , longer than remaining of interfrontal setae; b. Gaurax festivus, state 1 , shorter or as long as remaining of interfrontal setae. 


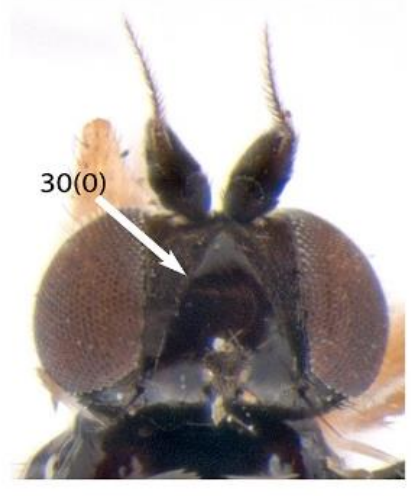

(a)

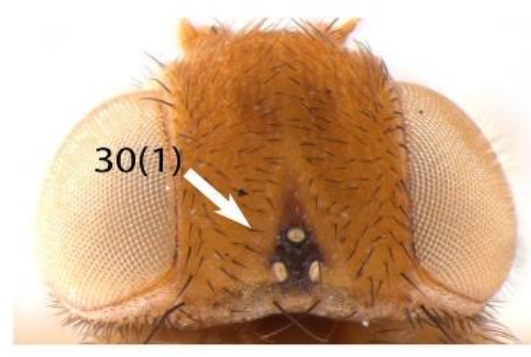

(b)

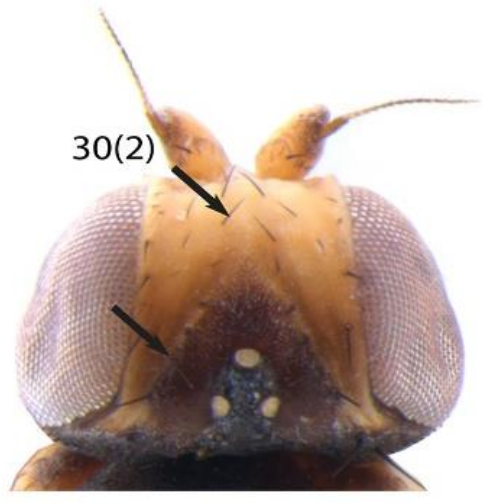

(c)

Figure 38a-c. Character 30, interfrontal setae relative to the frontal setae: a. Monochaetoscinella anonyma, state 0 , absent; b. Medeventor tschirnhausi, state 1 , present but as long as frontal setae; c. Dicraeus vagans, state 2 , conspicuous.

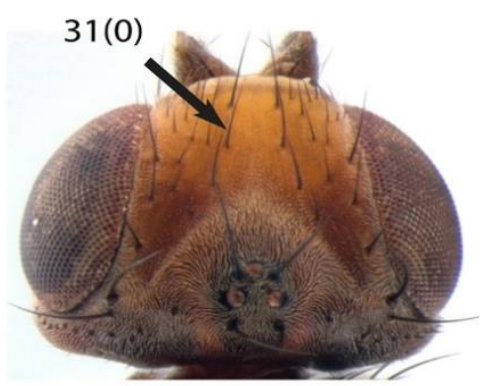

(a)

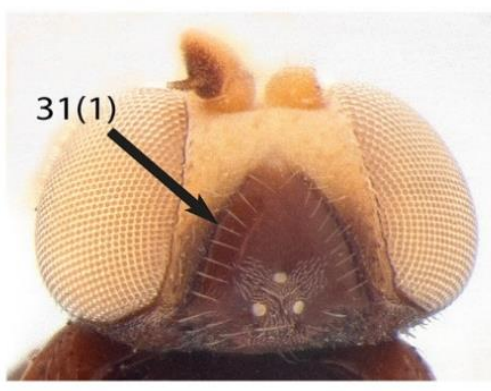

(b)

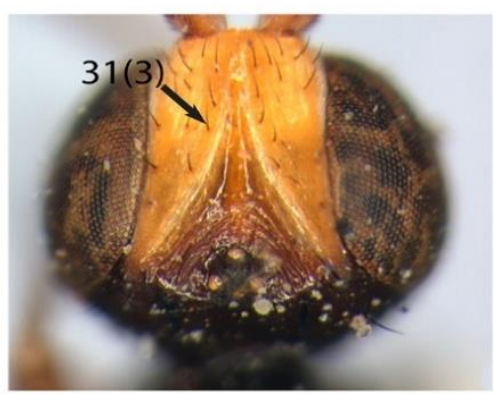

(d)

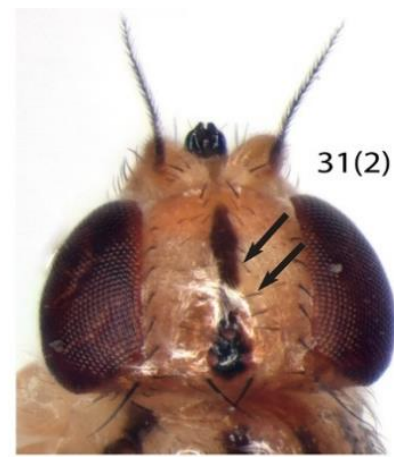

(c)

Figure 39a-d. Character 31, direction of interfrontal seta: a. Siphonellopsis lacteibasis, state 0 , all proclinate; b. Oscinisoma cognatum, state 1 , all inclinate; c. Metasiphonella sp., state 2, anteriorly proclinate and posteriorly inclinate; d. Sagareocerus aberrans, state 3, all straight. 


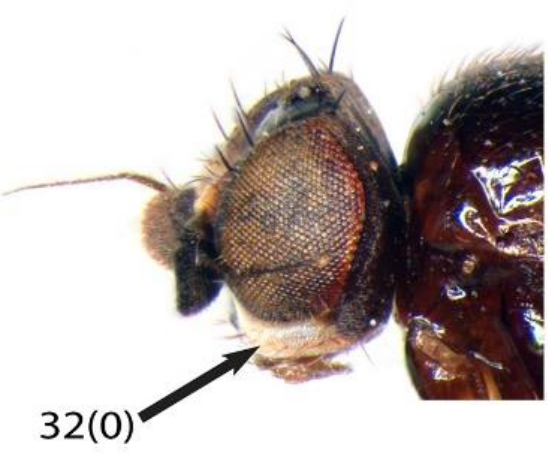

(a)

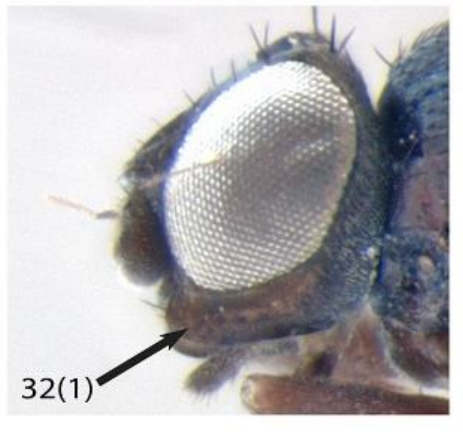

(b)

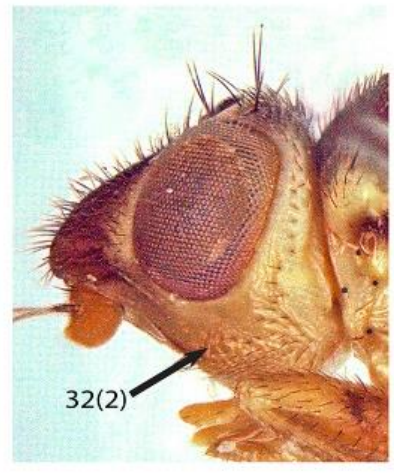

(c)

Figure 40a-c. Character 32, height of gena relative to the height of first flagellomere of antenna: a. Biorbitella hesperia, state 0, shorter; b. Malloewia diabolus, state 1, almost as long; c. Pseudeurina maculata (photography by Barbara Ismay), state 2, longer. 


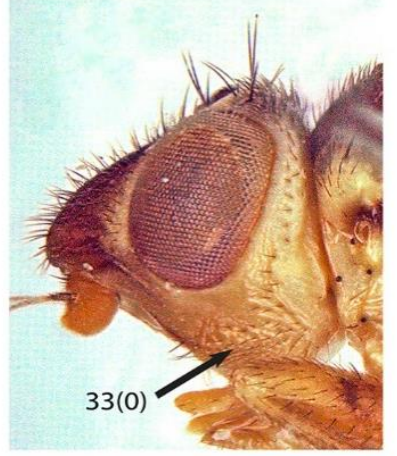

(a)

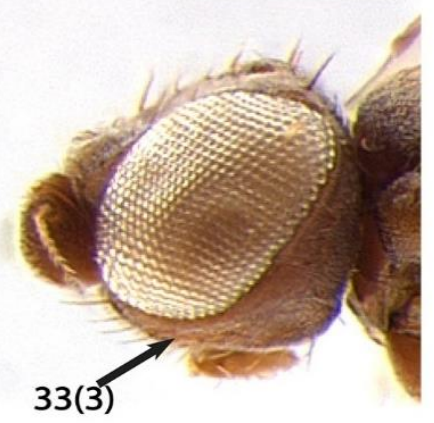

(d)

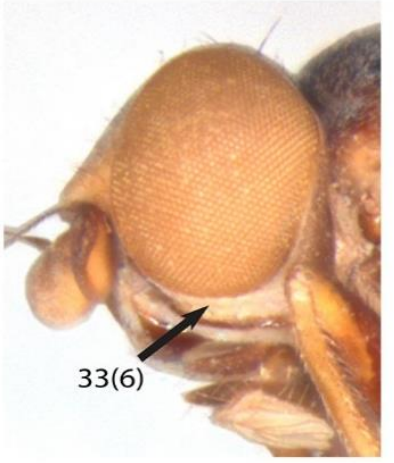

(g)

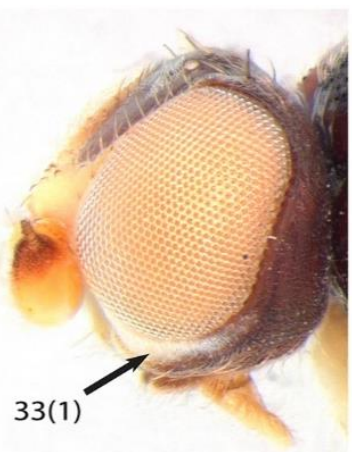

(b)

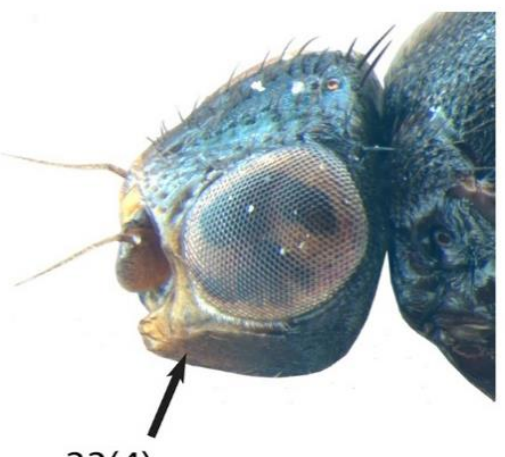

33(4)

(e)

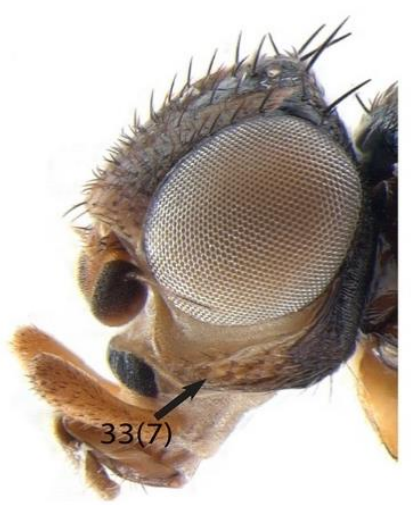

(h)

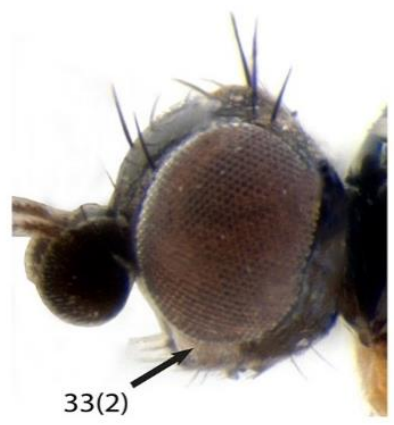

(c)

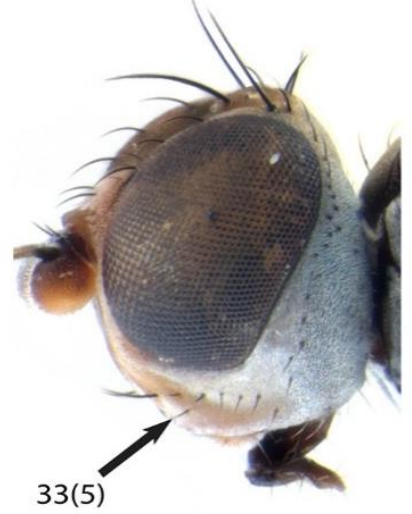

(f)

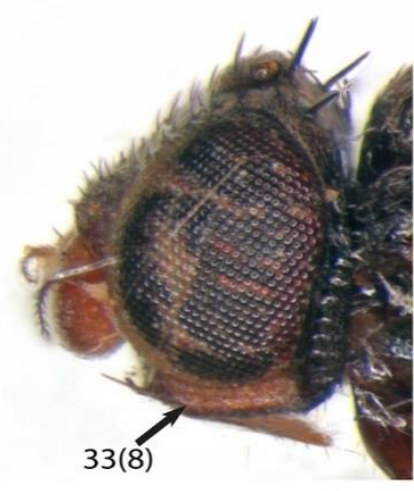

(i)

Figure 41a-i. Character 33, color of gena: a. Pseudeurina maculata (photography by Barbara Ismay), state 0 , all over yellow or light brown; b. Oscinisoma cognatum, state 1 , posterior half yellow; c. Monochaetoscinella anonyma, state 2, anterior half yellow; d. Eribolus hungaricus, state 3, dark color; e. Polyodaspis ruficornis, state 4, posterior third yellow; f. Siphonellopsis lacteibasis, state 5, anterior third yellow; g. Trigonomma coeruleifrons (Photography by Paula Raile Riccardi), state 6, posterior two third yellow; h. Lasiambia palposa, state 7, anterior two third yellow; i. Loxobathmis obliquans, state 8 , all over yellow except anterior tip dark. 


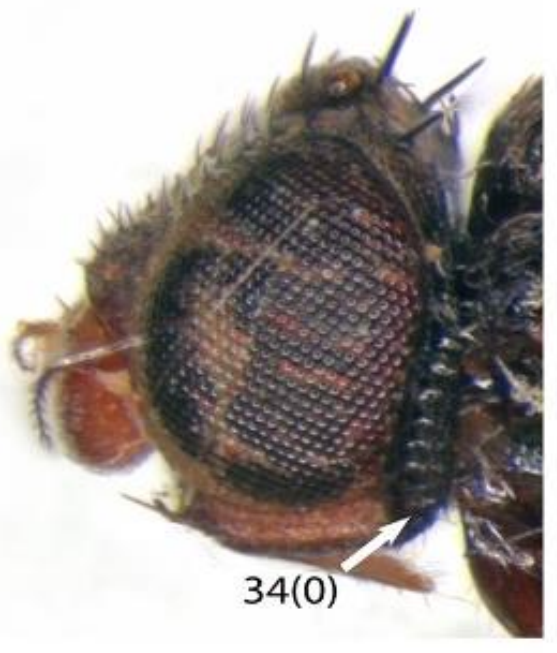

(a)

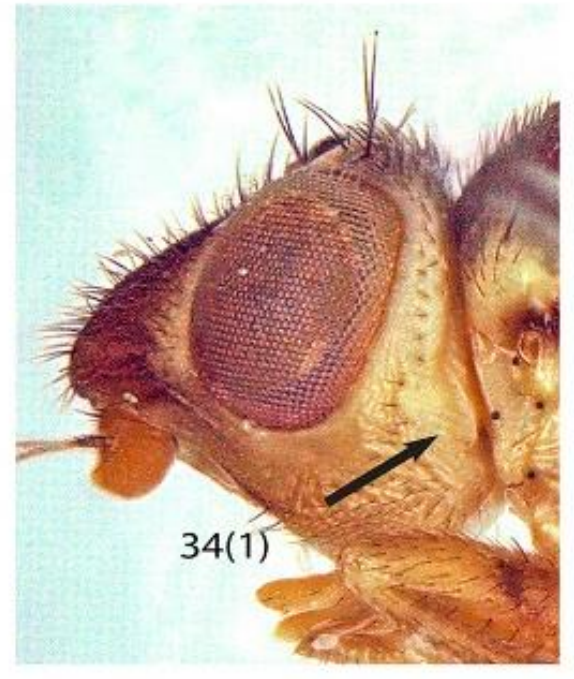

(b)

Figure 42a-b. Character 34, color of postgena: a. Loxobathmis obliquans, state 0 , black or dark brown; b. Pseudeurina maculata (photography by Barbara Ismay), state 1, yellow or light brown.

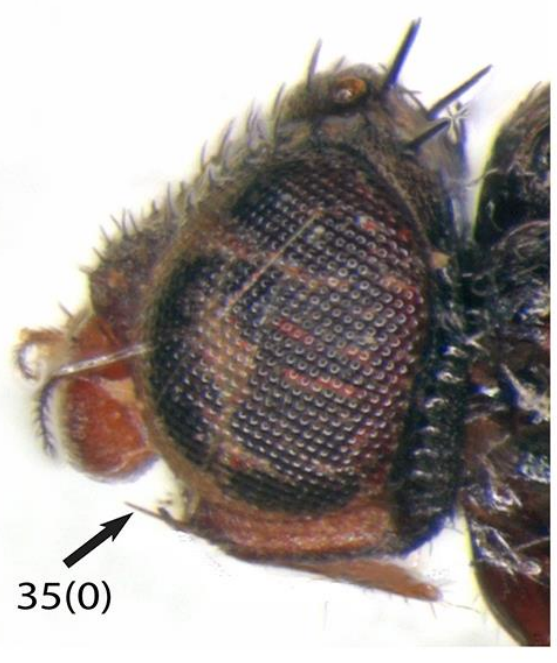

(a)

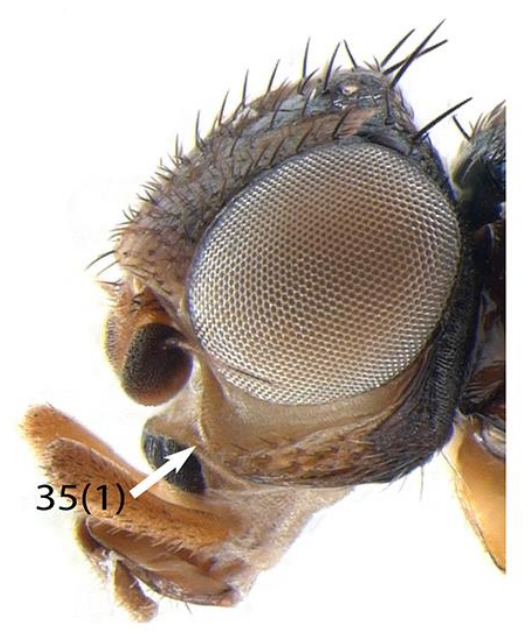

(b)

Figure 43a-b. Character 35, vibrissal seta: a. Loxobathmis obliquans, state 0 , present; b. Lasiambia palposa, state 1 , absent. 


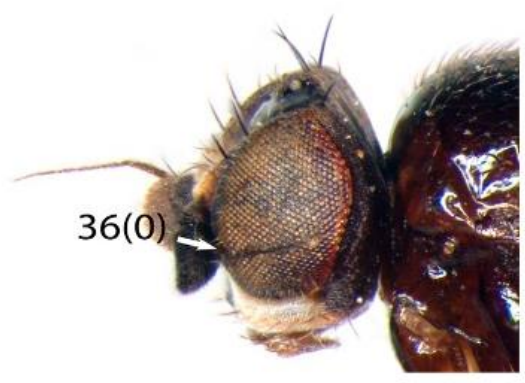

(a)

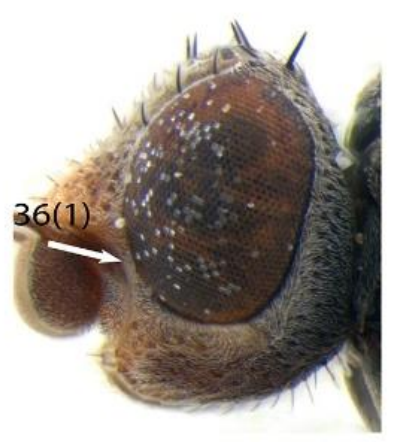

(b)

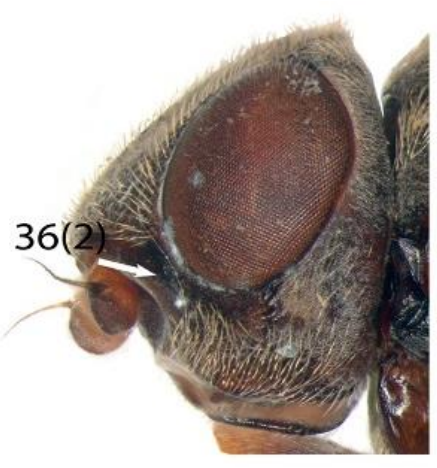

(c)

Figure 44a-c. Character 36, parafacialia (related to the broadness of first flagellomere of antenna): a. Biorbitella hesperia, state 0 , absent; b. Aphanotrigonum trilineatus, state 1, narrow; c. Lipara lucens, state 2 , wider.

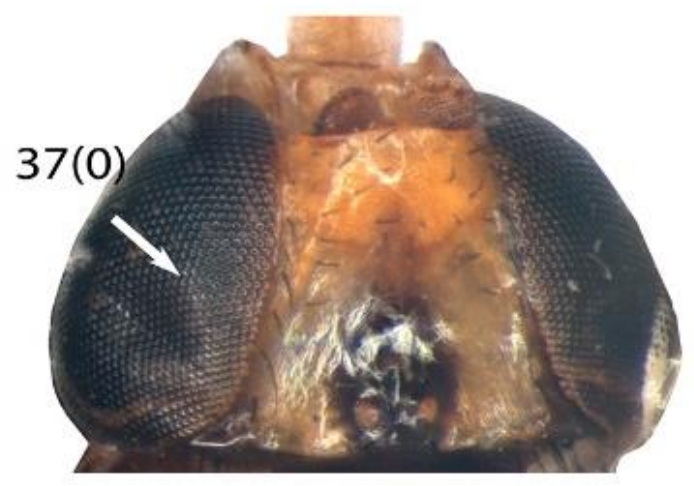

(a)

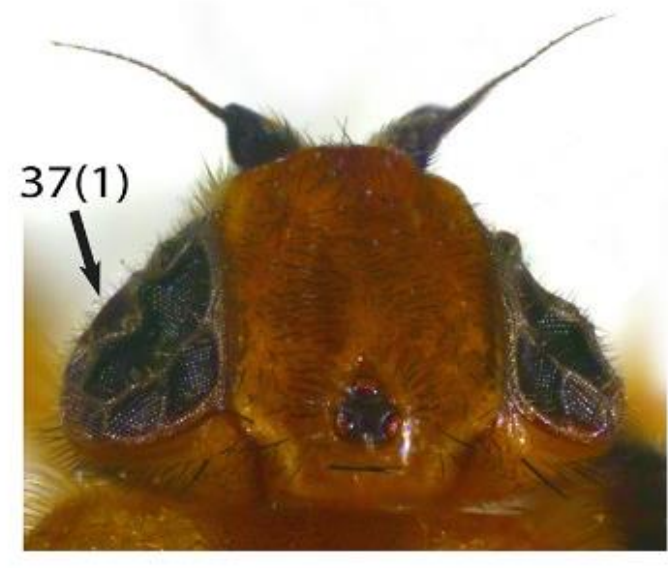

(b)

Figure 45a-b. Character 37, pilosity of eye: a. Heteroscinis hystrix, state 0, bare; b. Batrachomyia nigritarsis, state 1 , hairy. 


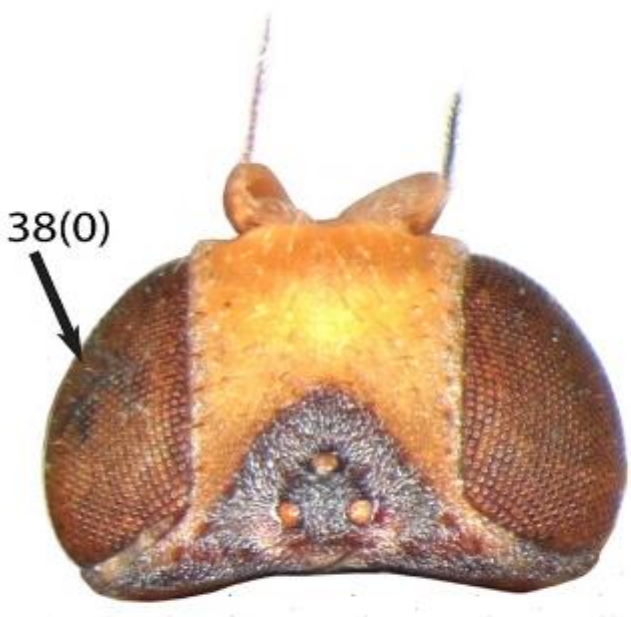

(a)

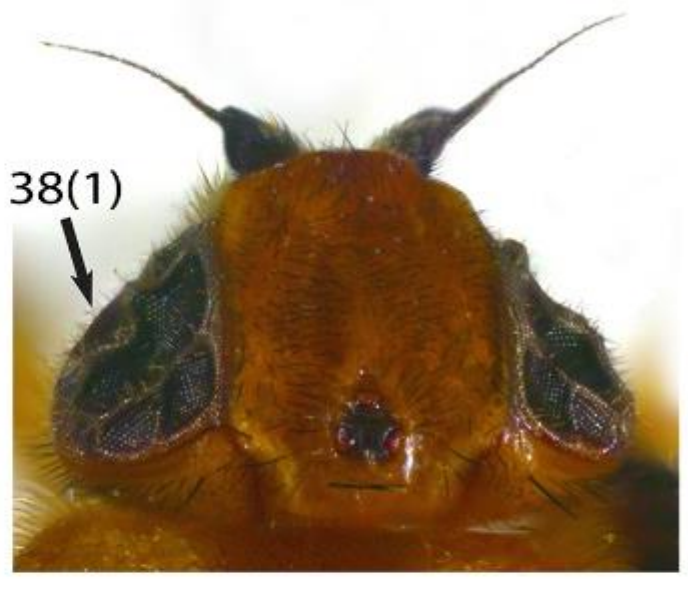

(b)

Figure 46a-b. Character 38, dispersal pilosity of eye: a. Hippelates plebejus, state 0 , scattered; b. Batrachomyia nigritarsis, state 1 , dense.

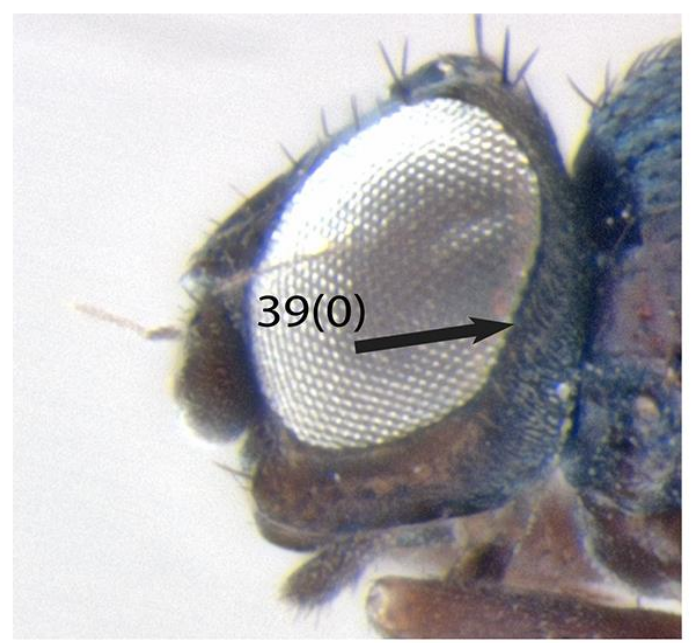

(a)

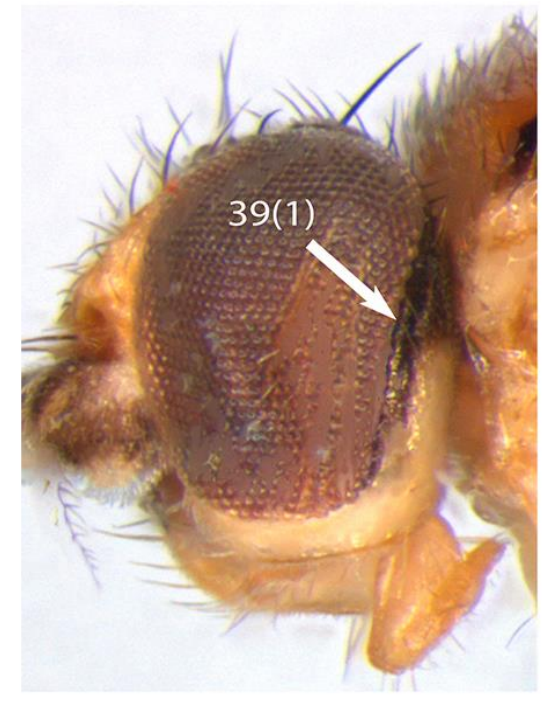

(b)

Figure 47a-b. Character 39, posterior margin of eye, 1/4 ventral: a. Malloewia diabolus, state 0 , continues; b. Gaurax festivus, state 1, with notch. 


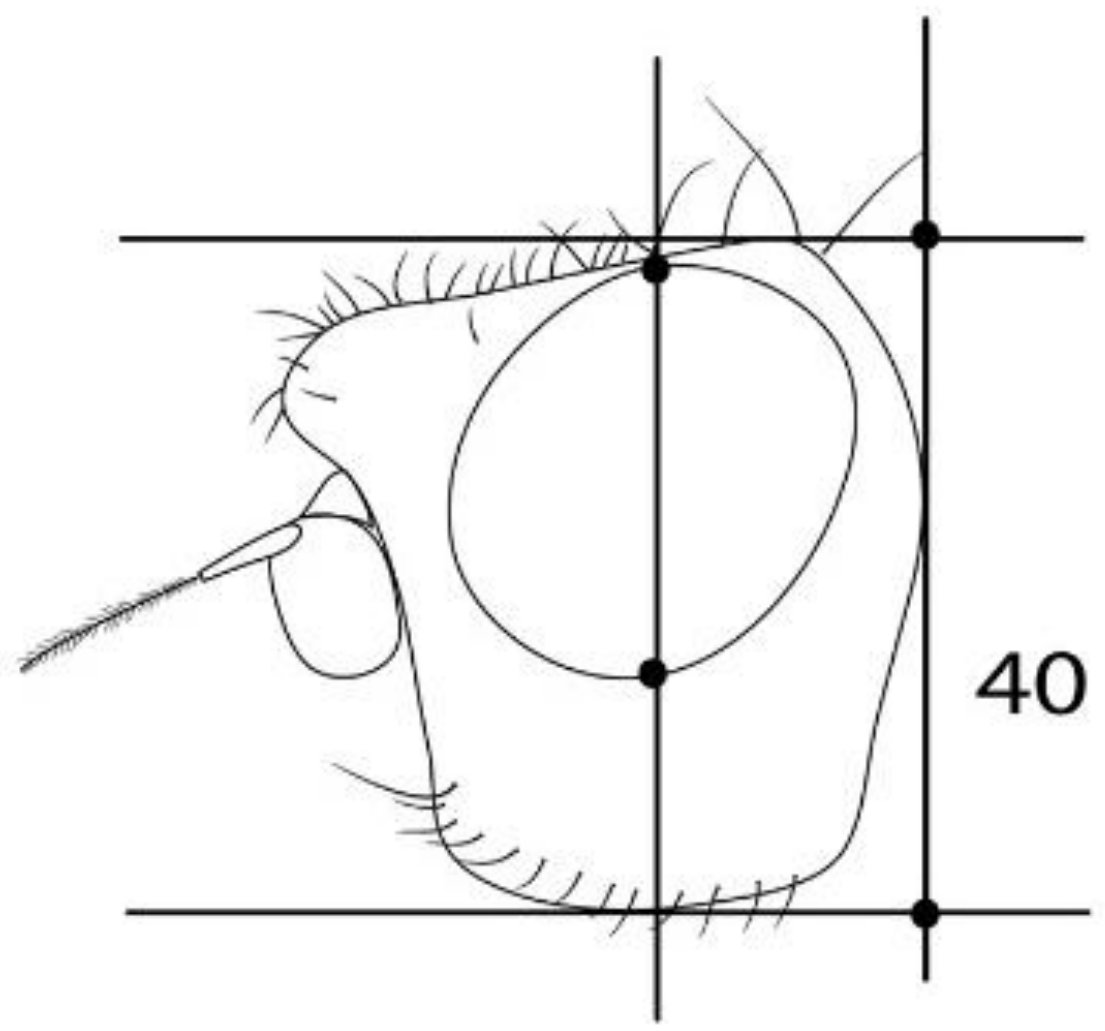

Figure 48: Character 40, rate of eye compared with head capsule vertically in lateral view: Schematic shape.

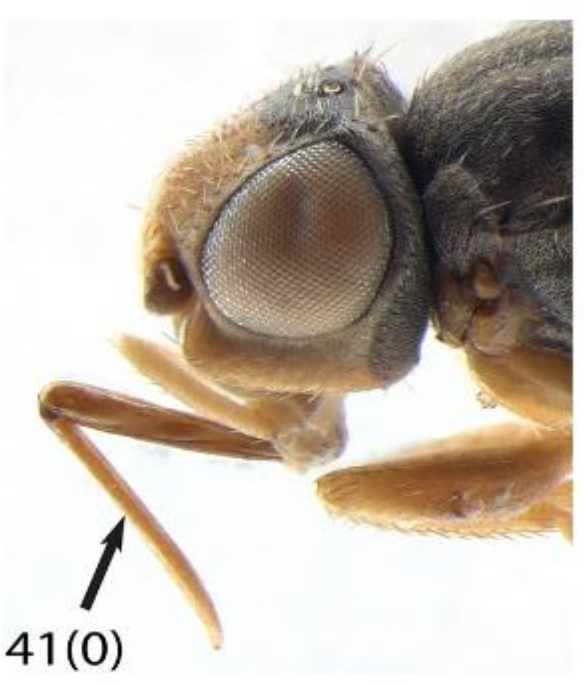

(a)

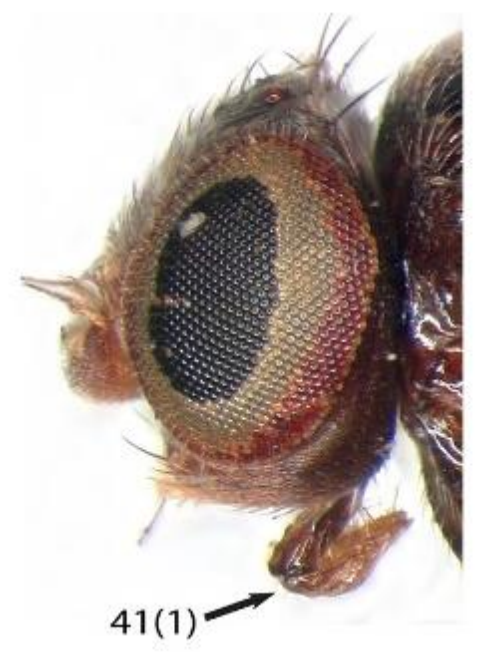

(b)

Figure 49a-b. Character 41, proboscis: a. Oscinimorpha albisetosa, state 0, geniculate; b. Speccafrons mallochi, state 1 , not geniculate. 


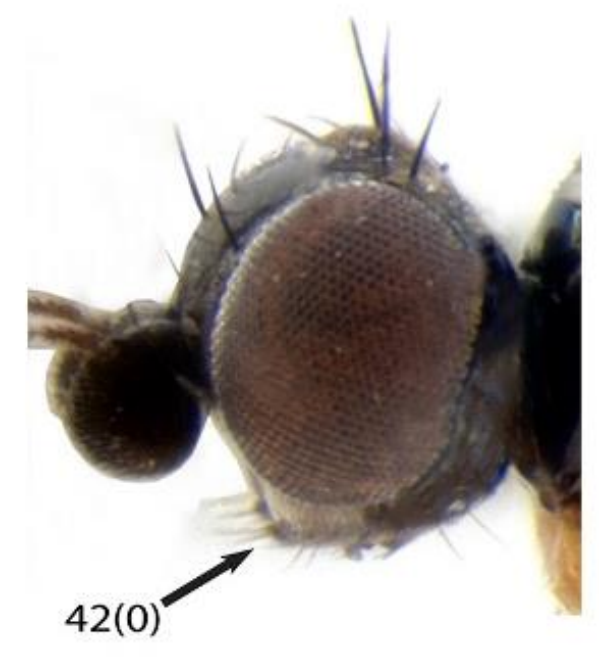

(a)

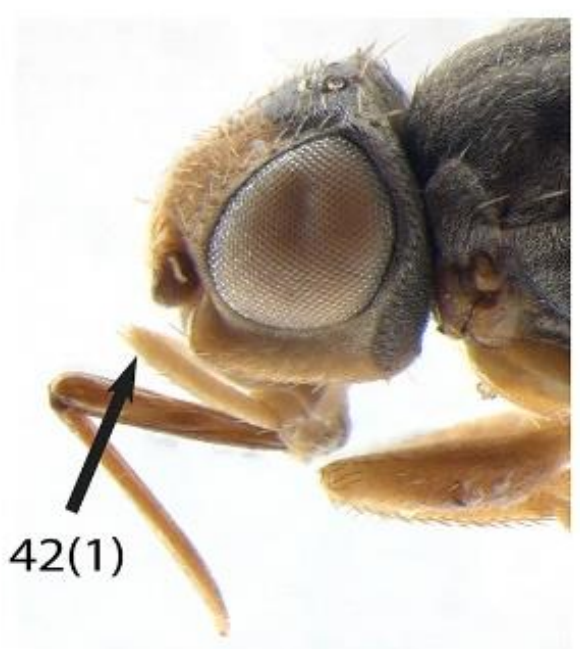

(b)

Figure 50a-b. Character 42, length of palpus in comparison with the oral cavity (lower margin of the face): a. Monochaetoscinella anonyma, state 0 , at most at the margin of oral cavity; $b$. Oscinimorpha albisetosa, state 1, longer than the margin of oral cavity.

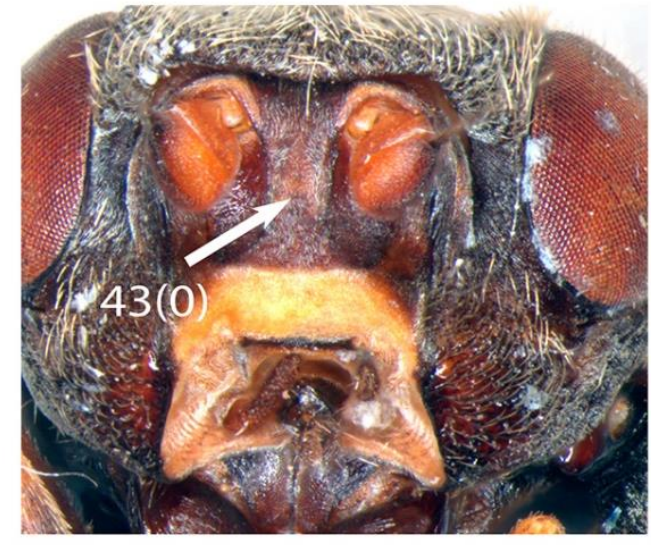

(a)

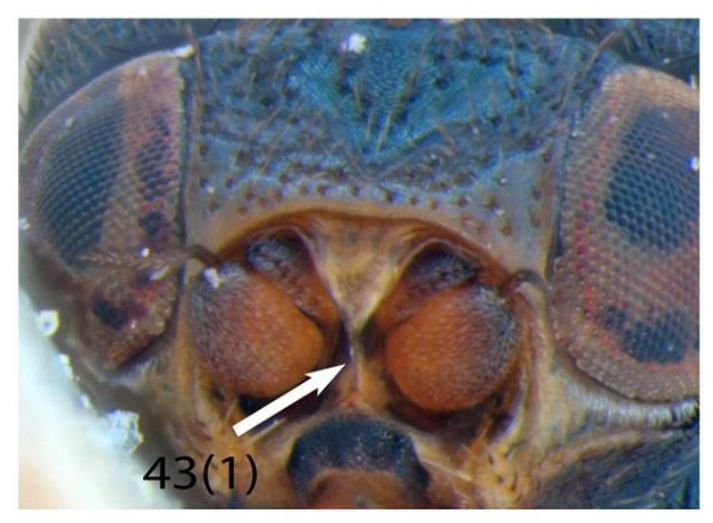

(b)

Figure 51a-b. Character 43, shape of facial carina: a. Lipara lucens, state 0 , wide dorsally and ventrally; b. Polyodaspis ruficornis, state 1 , narrow ventrally and wide or narrow dorsally. 


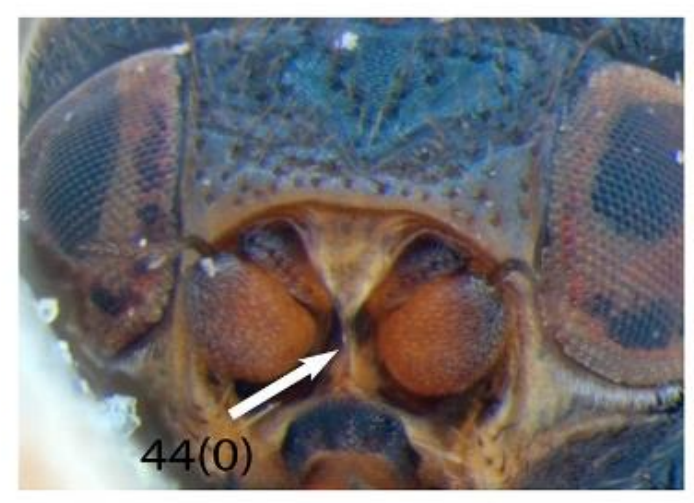

(a)

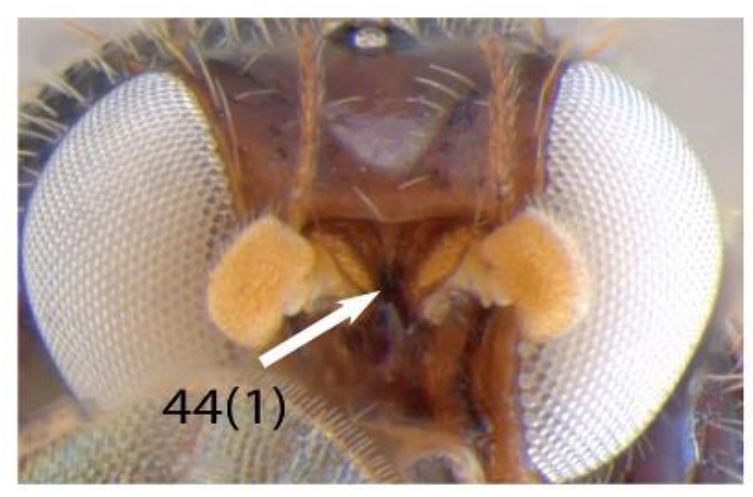

(b)

Figure 52a-b. Character 44, facial carina: a. Polyodaspis ruficornis, state 0 , complete, reaching to the epistomal margin or very close; b. Rhodesiella sp.1, state 1, incomplete, extending almost halfway to epistomal margin.

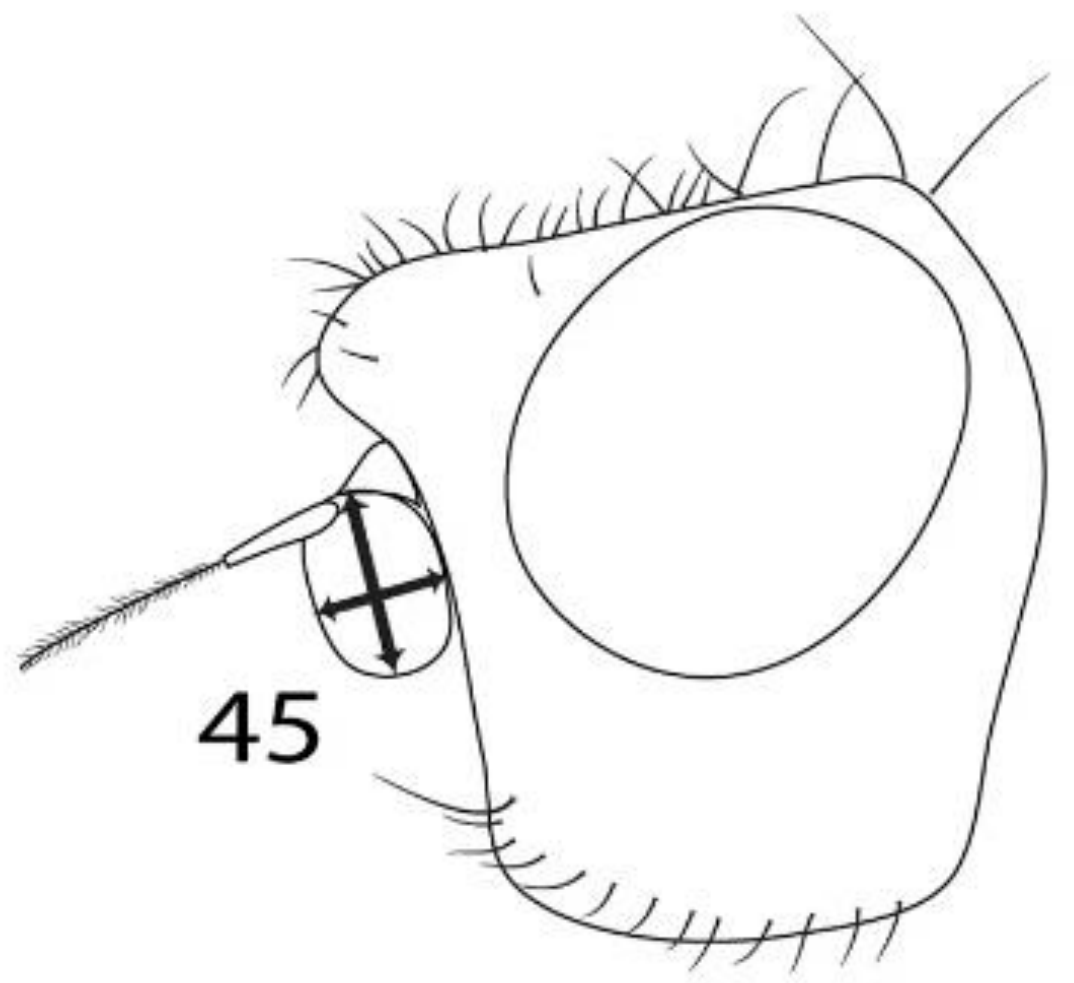

Figure 53: Character 45, rate of first antennal flagellomere (length/wide): Schematic shape. 


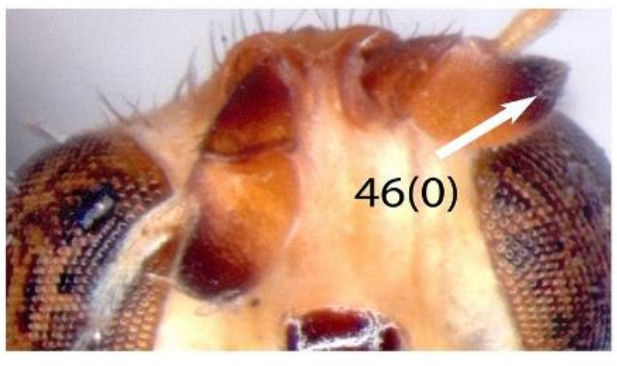

(a)

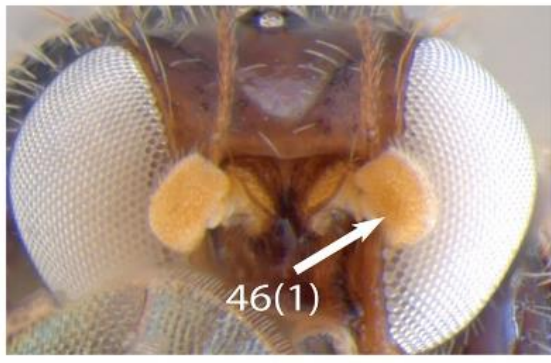

(b)

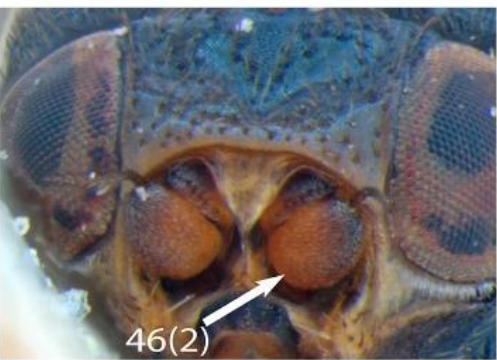

(d)

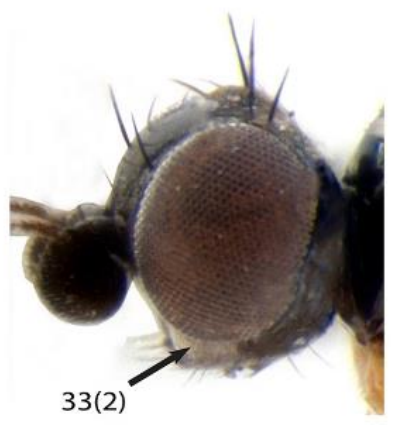

(c)

Figure 54a-d. Character 46, first antennal flagellomere distally: a. Diplotoxa versicolor, state 0 , one b. Rhodesiella sp.1, state 1, between 1.1 and 1.5; c. Monochaetoscinella anonyma, state 2, more than 1.5 ; d. Polyodaspis ruficornis, state 2. 


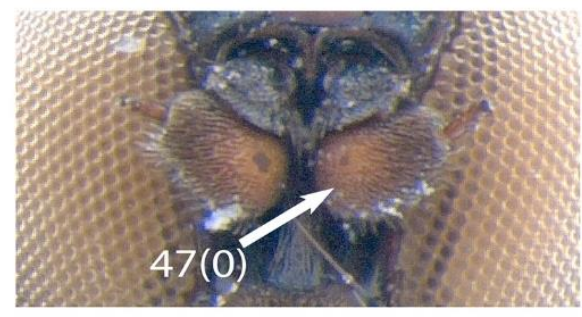

(a)

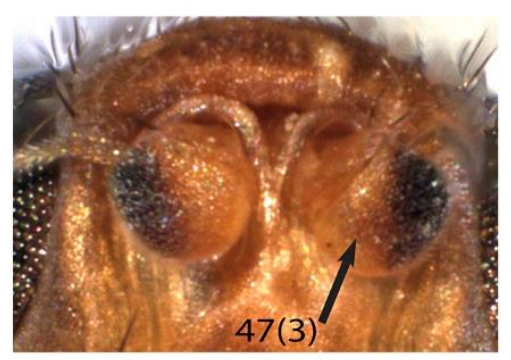

(d)

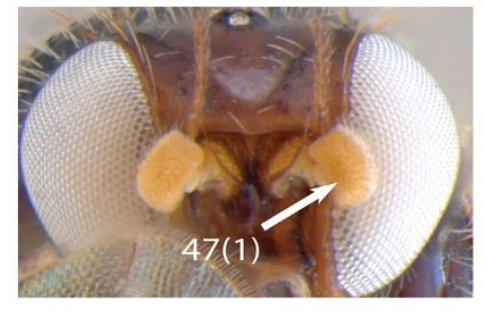

(b)

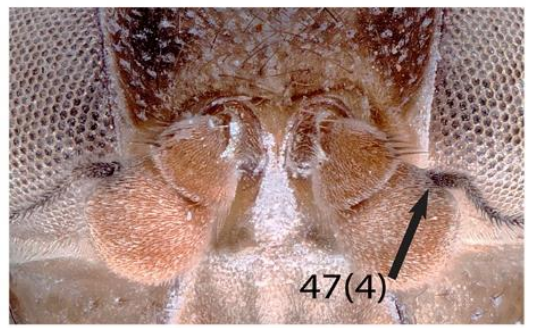

(e)

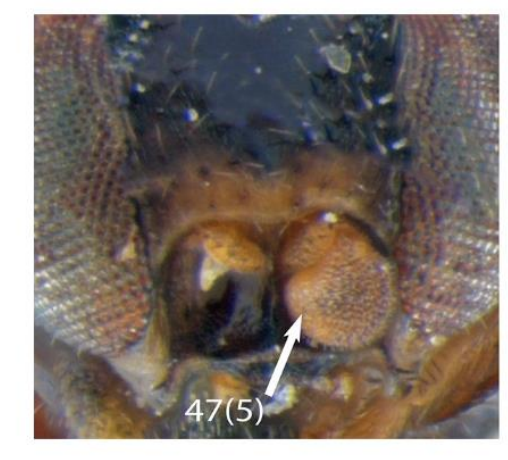

(g)

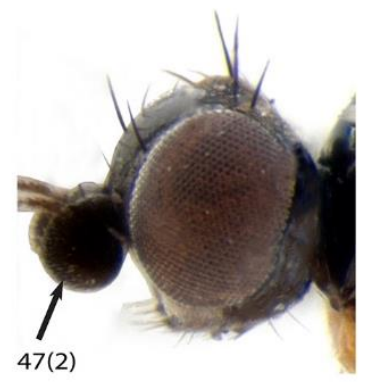

(c)

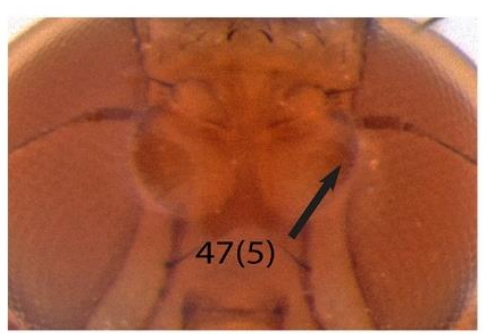

(f)

Figure 55a-g. Character 47, color of first antennal flagellomere: a. Rhodesiella sp.2, state 0 , posterior half yellow or light; b. Rhodesiella sp.1, state 1, entirely yellow or light brown; c. Monochaetoscinella anonyma, state 2, entirely dark brown or black; d. Opetiophora straminea, state 3, posterior two third yellow or light brown; e. Medeventor nubosus (Photography by Canadian National Collection Museum), state 4, entirely yellow or light brown except at base of arista; f. Notaulacella sp., state 5, yellow or light brown, dark dorsobasally; g. Lioscinella sulfurihalterata, state 5 . 


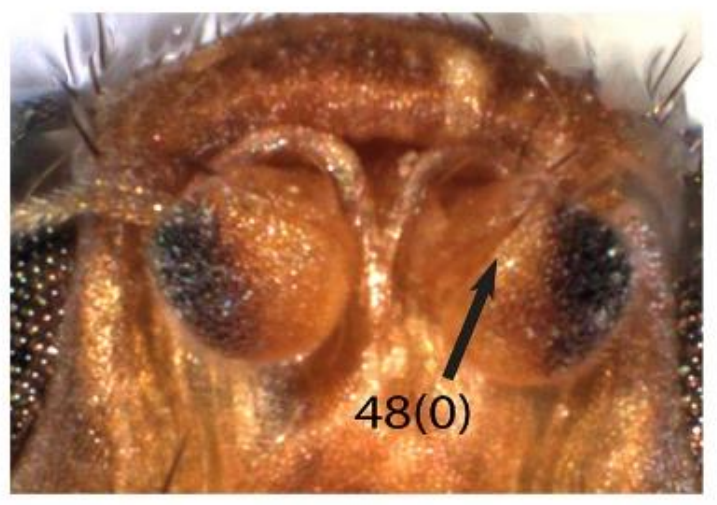

(a)

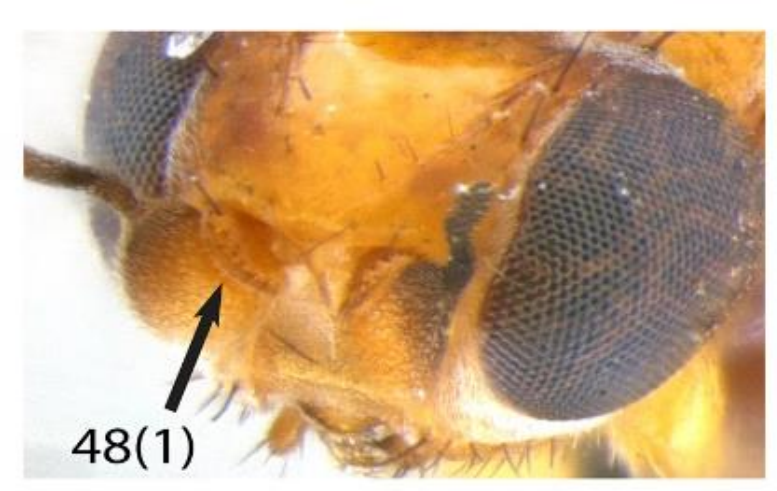

(b)

Figure 56a-b. Character 48, posterior end of first antennal flagellomere, in frontal view: a. Opetiophora straminea, state 0 , straight; b. Elachiptera brevipennis, state 1 , concave (reniform).

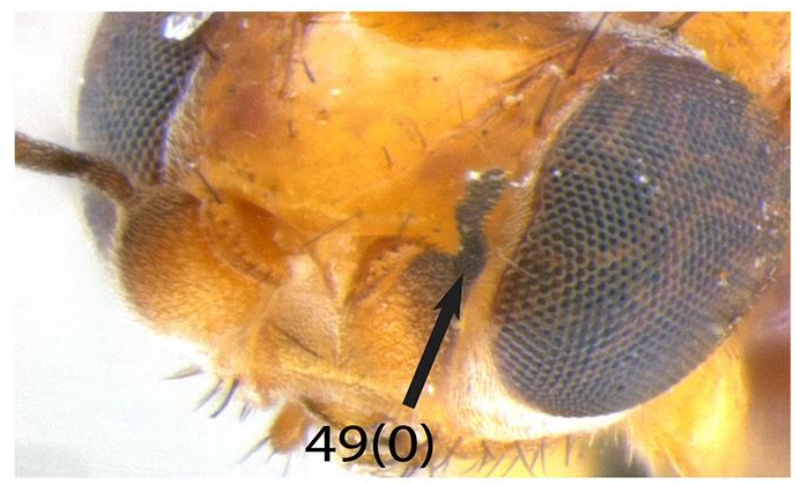

(a)

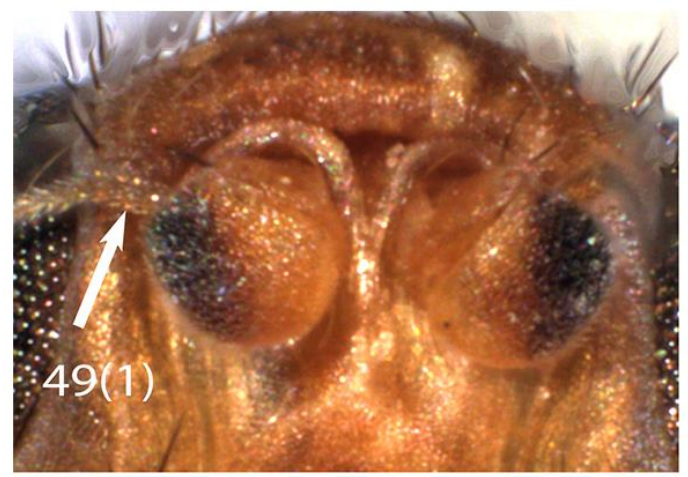

(b)

Figure 57a-b. Character 49, color of basal segment of arista: a. Elachiptera brevipennis, state 0 , dark; b. Opetiophora straminea, state 1 , yellow. 


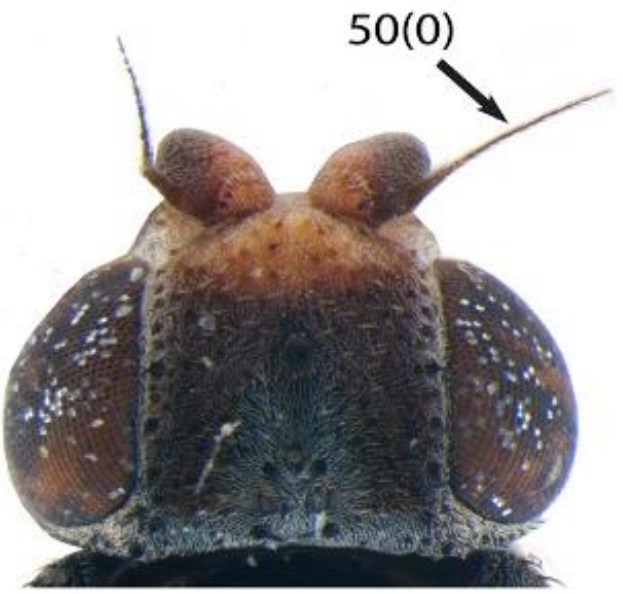

(a)

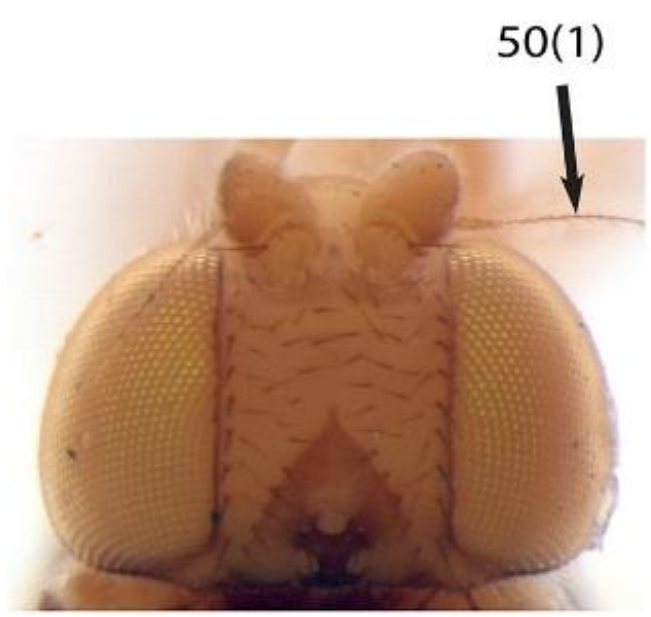

(b)

Figure 58a-b. Character 50, color of distal segment of arista: a. Aphanotrigonum trilineatus, state 0 , dark; b. Eugaurax sp., state 1, yellow.

$$
51(0)
$$

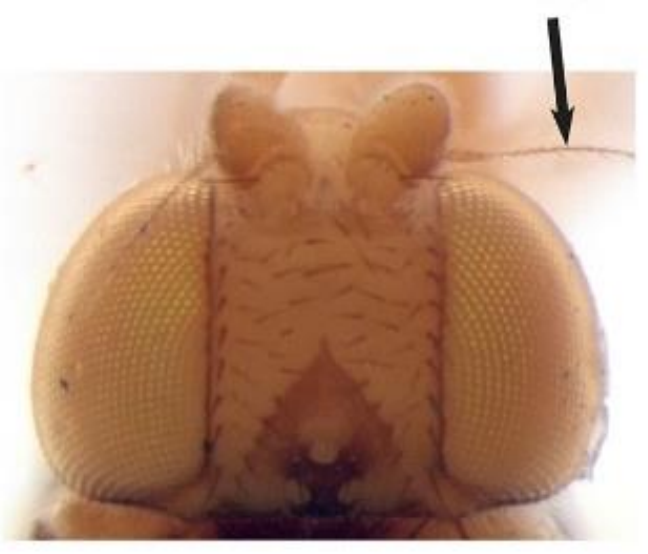

(a)

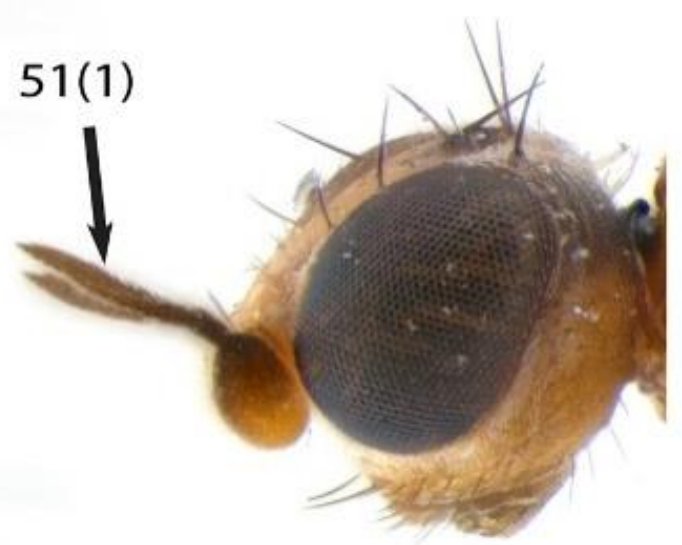

(b)

Figure 59a-b. Character 51, width of distal half arista: a. Eugaurax sp., state 0, thin; b. Elachiptera brevipennis, state 1 , thick. 


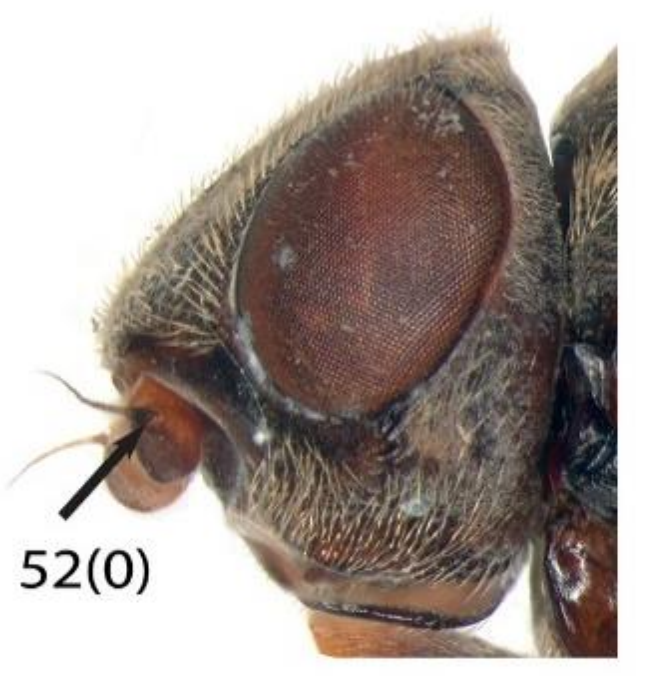

(a)

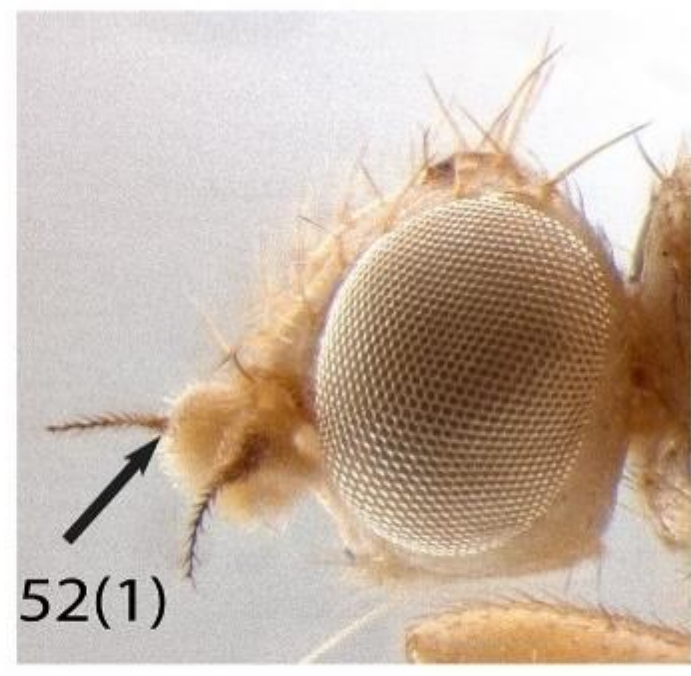

(b)

Figure 60a-b. Character 52, position of arista on first antennal flagellomere: a. Lipara lucens, state 0 , placed at most equal with arista base; b. Cadrema pallida, state 1 , more than arista base, at least twice.

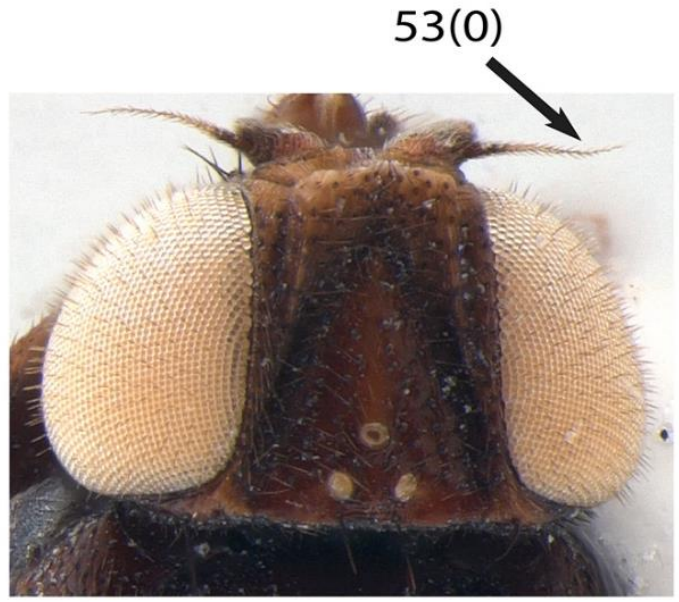

(a)

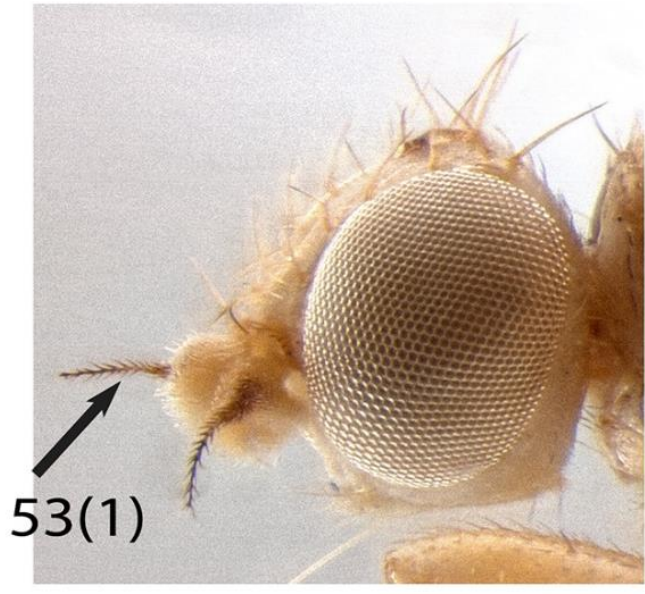

(b)

Figure 61a-b. Character 53, pilosity of entire arista comparing with the stem of arista: a. Calamoncosis minima, state 0 , short hairs; b. Cadrema pallida, state 1 , long hairs. 


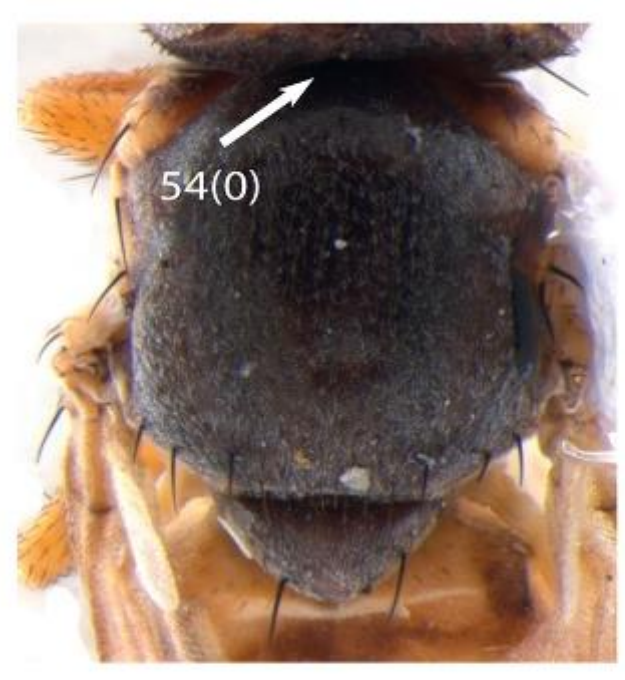

(a)

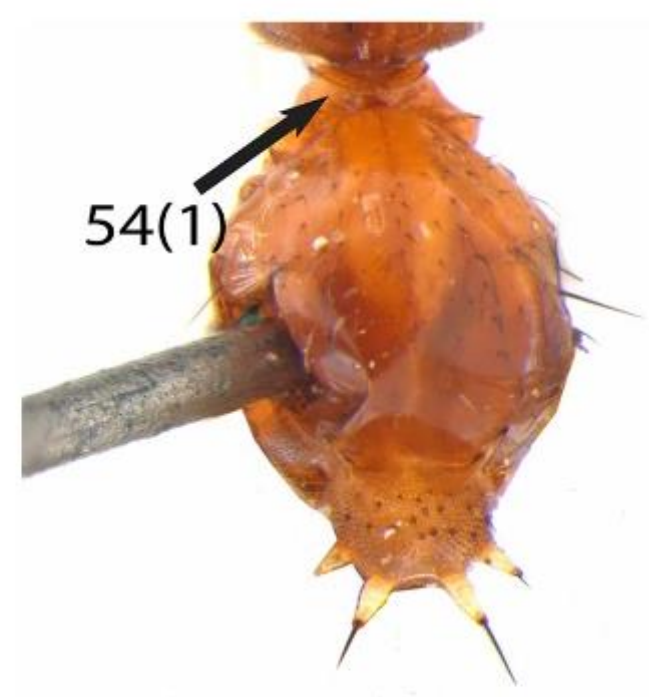

(b)

Figure 62a-b. Character 54, anterior part of mesonotum: a. Dicraeus vagans, state 0 , not projected; b. Sepsidoscinis maculipennis, state 1, projected (neck form).

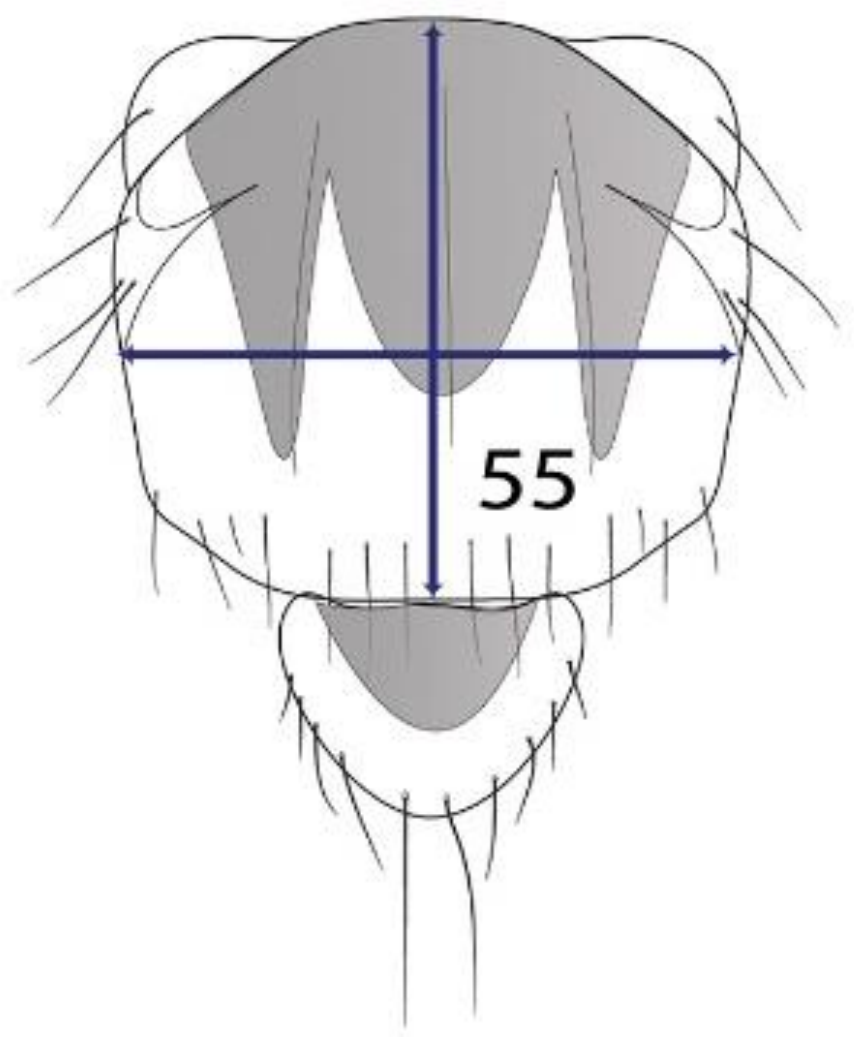

Figure 63: Character 55, length of scutum dorsally (width/length): Schematic shape. 


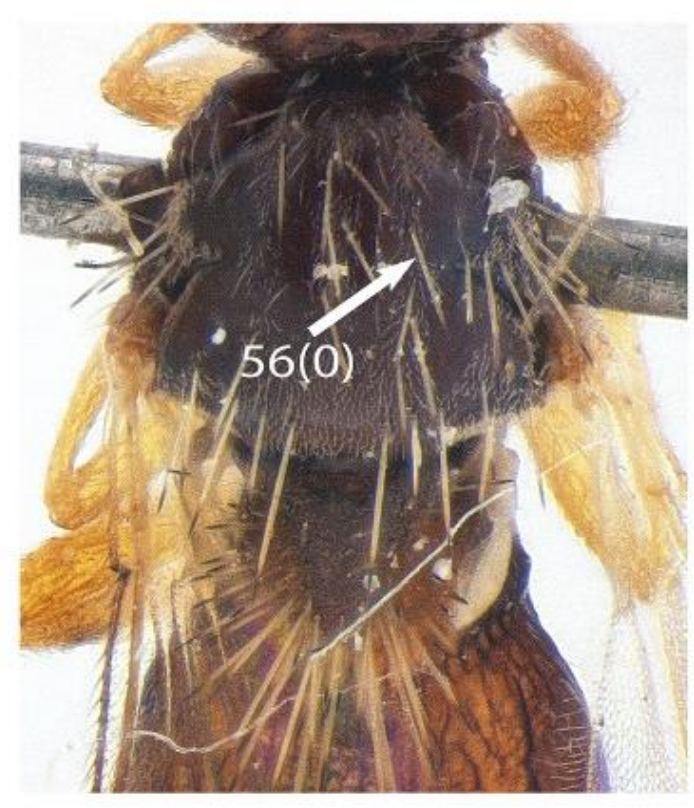

(a)

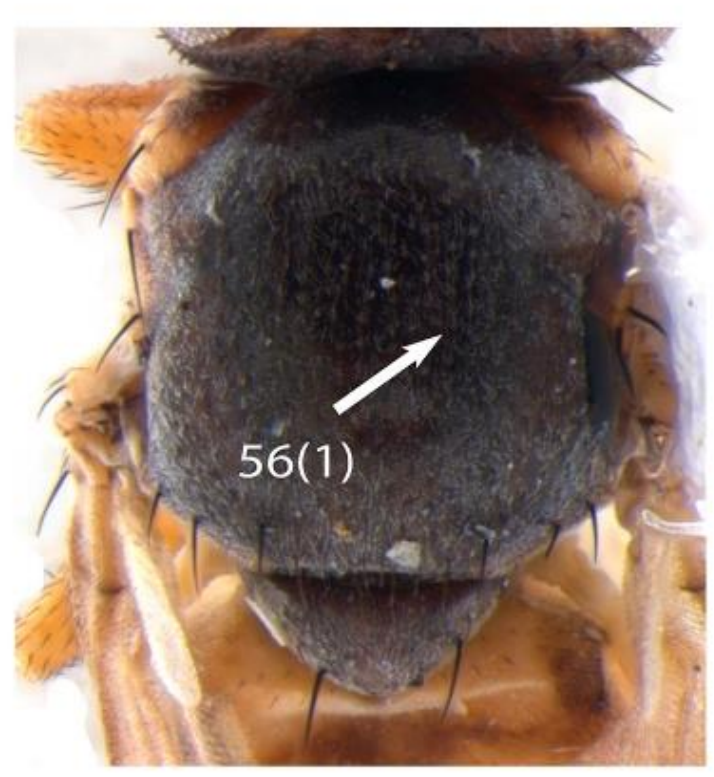

(b)

Figure 64a-b. Character 56, acrostical seta: a. Anatrichus erinaceus, state 0, present; b. Dicraeus vagans, state 1 , absent.

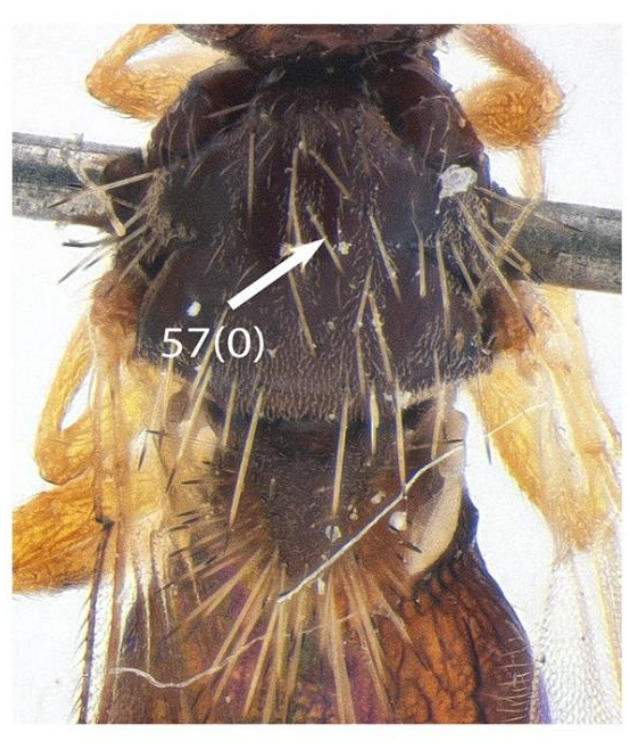

(a)

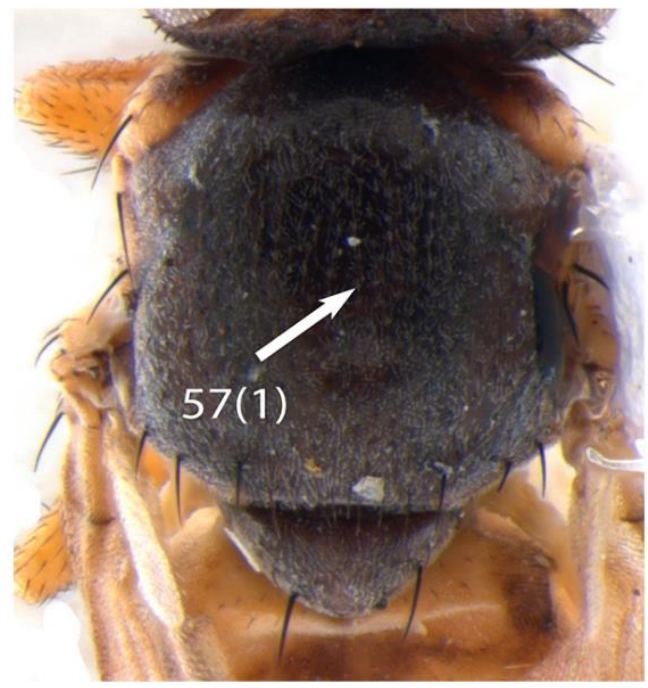

(b)

Figure 65a-b. Character 57, dorsocentral seta: a. Anatrichus erinaceus, state 0, present; b. Dicraeus vagans, state 1 , absent. 


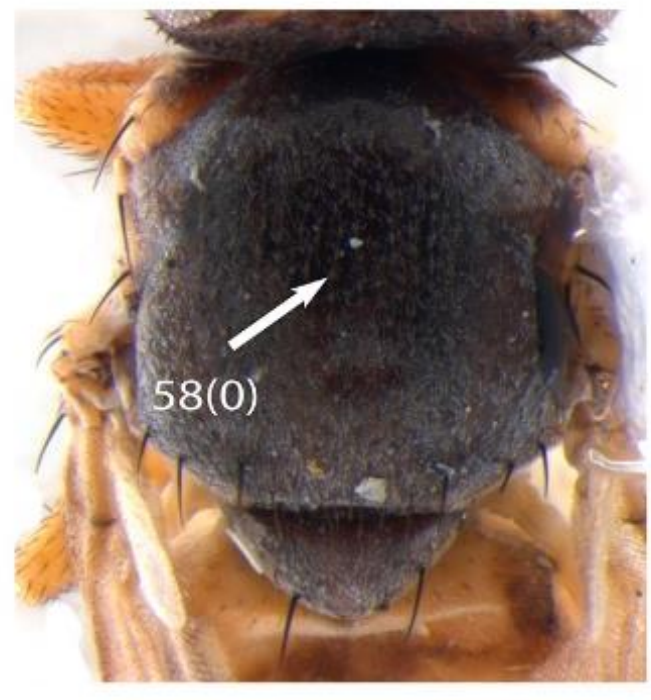

(a)

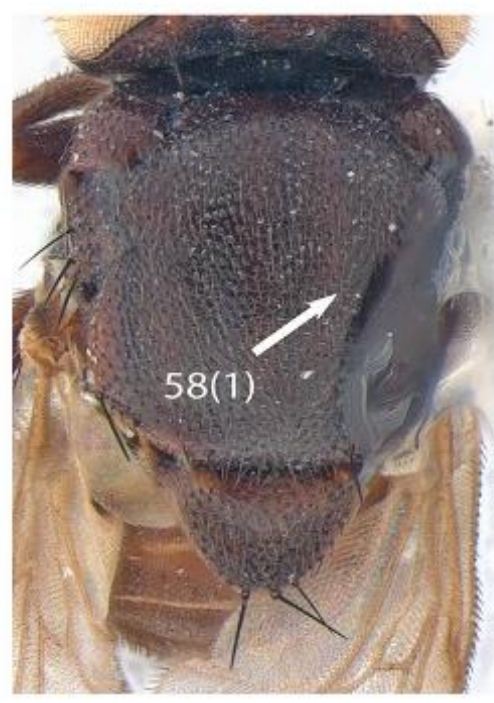

(b)

Figure 66a-b. Character 58, pruinosity all over the scutum: a. Dicraeus vagans, state 0 , present; b. Calamoncosis minima, state 1 , absent.

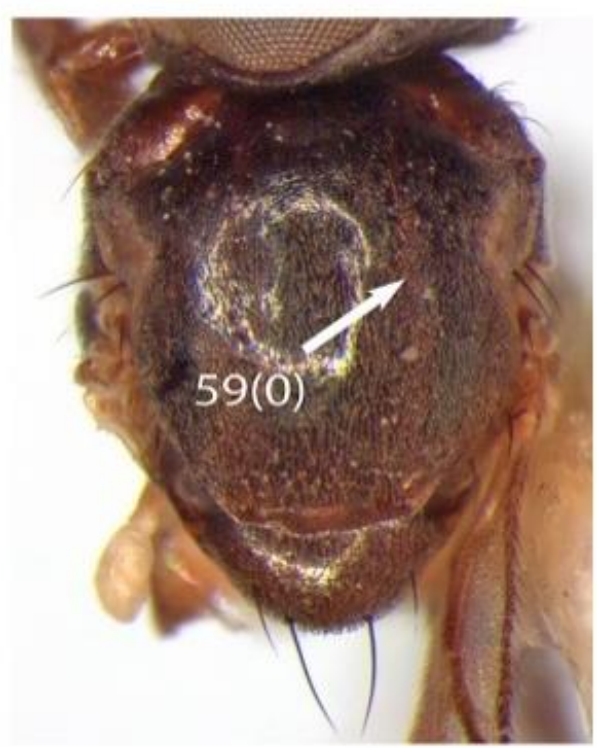

(a)

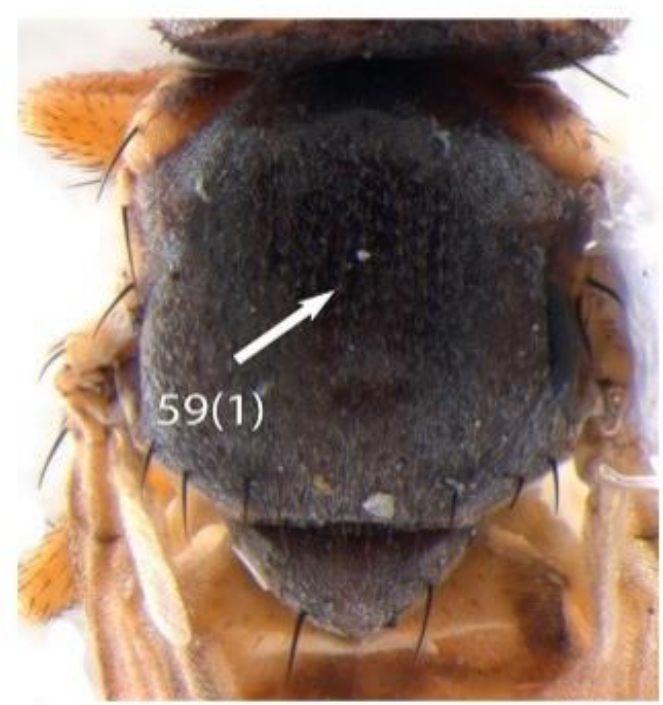

(b)

Figure 67a-b. Character 59, color of pruinosity on scutum: a. Apallates dissidens, state 0 , dark brown or black; b. Dicraeus vagans, state 1, yellow or whitish. 


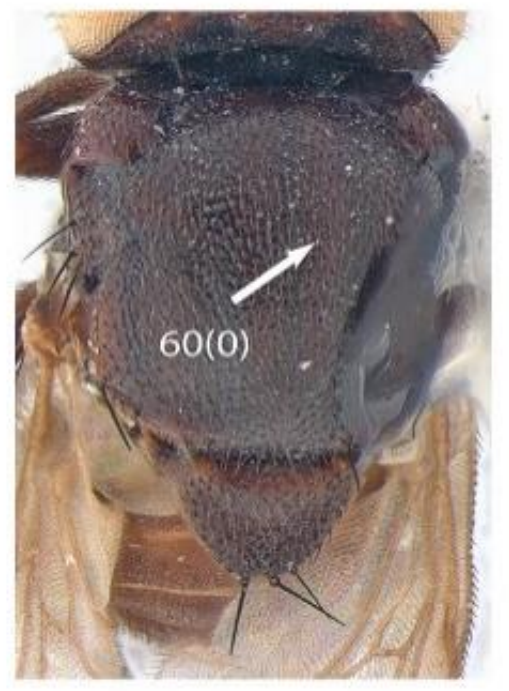

(a)

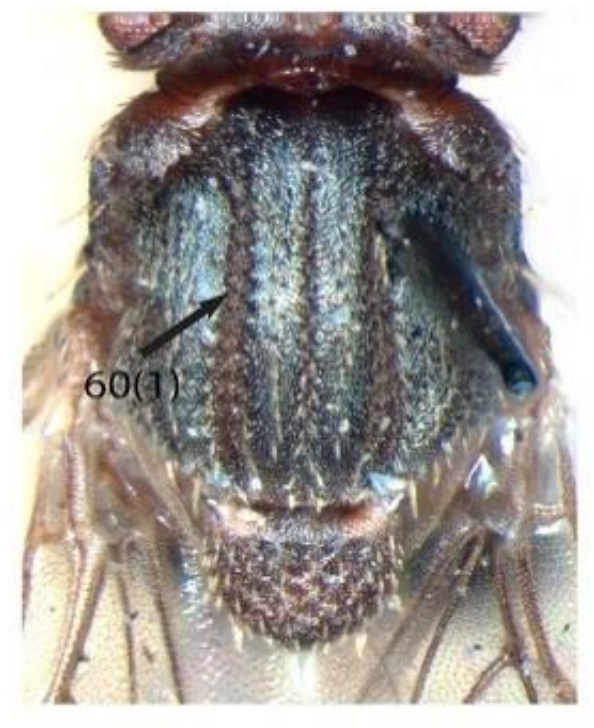

(b)

Figure 68a-b. Character 60, scutum: a. Calamoncosis minima, state 0 , no longitudinal sulcus; b. Tricimba lineella, state 1, with 1-3 longitudinal sulcus. 


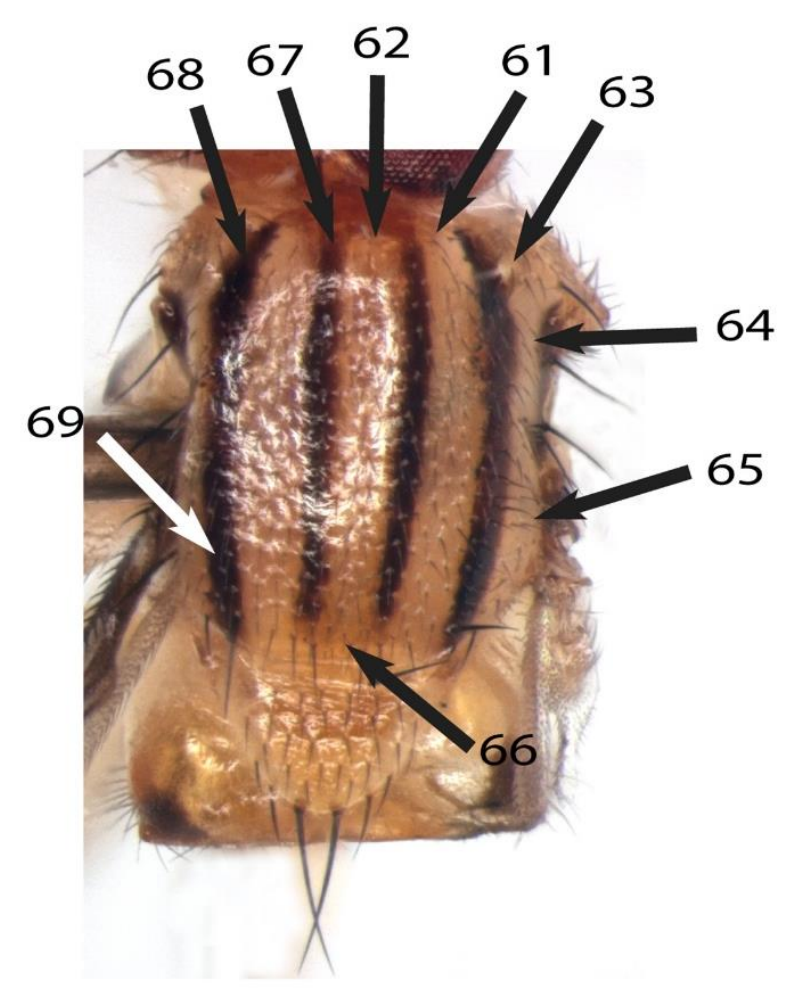

(a)

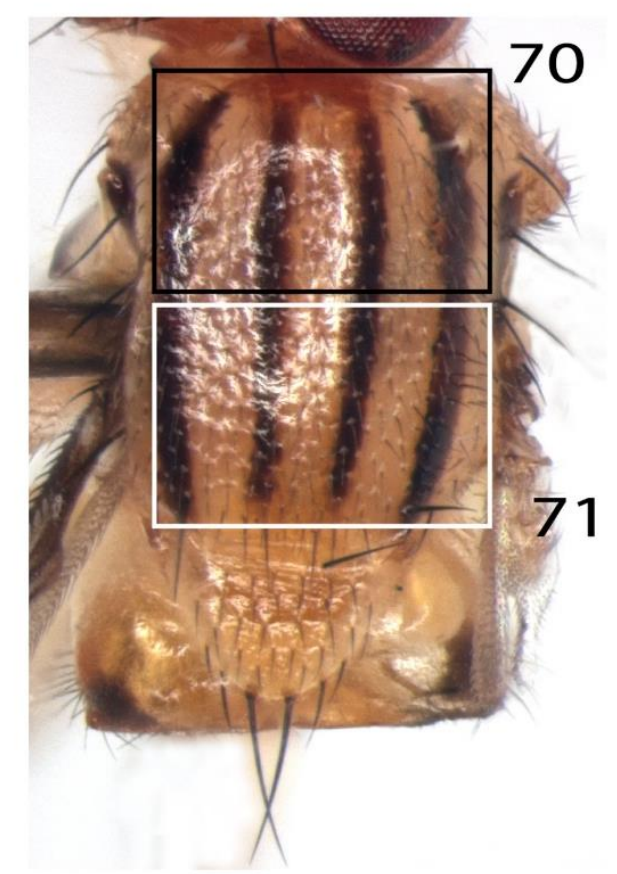

(b)

Figure 69a-b. Characters 61-71, areas over the scutum: a,b. Metasiphonella sp., C61, area over dc; C62, area over ac; C63, lateral area, dorsal to margin on scutum; C64, lateral most area on scutum; C65, scutum dorsal to wing base; C66, scutum posterior end; C67, scutum area between ac and dc; C68, scutum area, external line to the dorso-lateral to dc; C69, scutum area, external line to the ventro-lateral to dc; C70, scutum anterior half; C71, scutum area posterior half. 


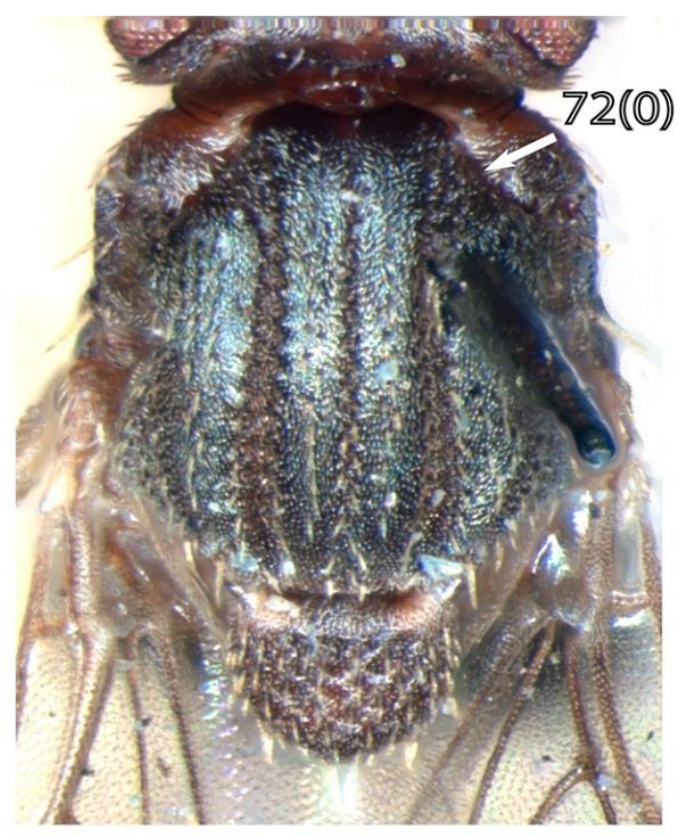

(a)

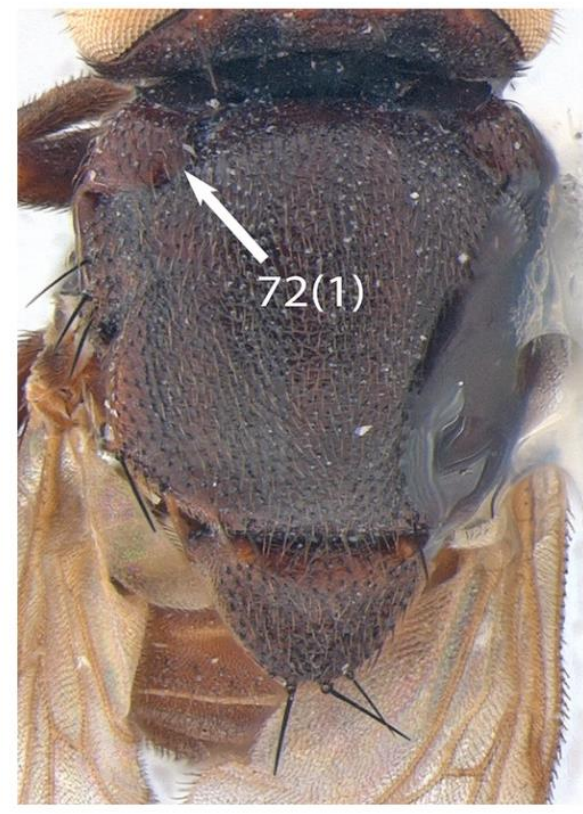

(b)

Figure 70a-b. Character 72, propleuron: a. Tricimba lineella, state 0 , well developed; $b$. Calamoncosis minima, state 1 , weakly developed.

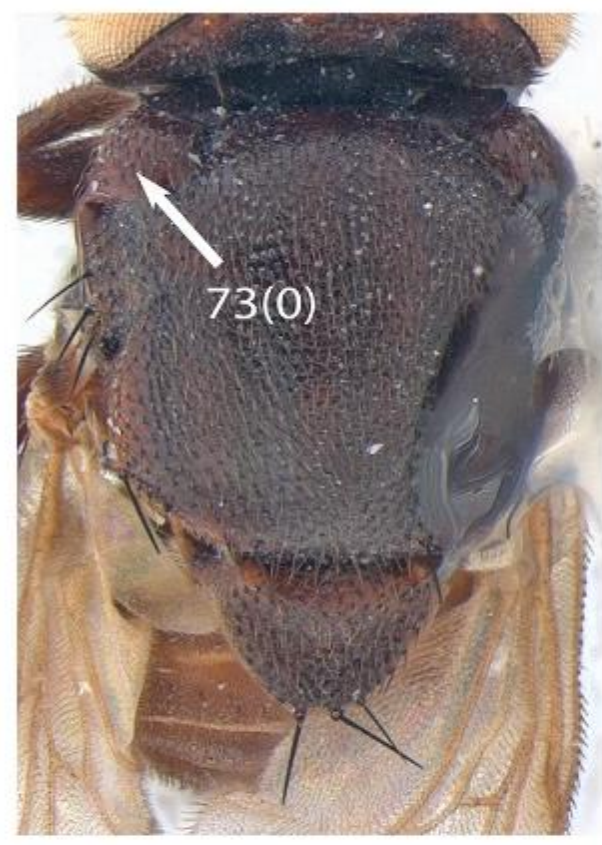

(a)

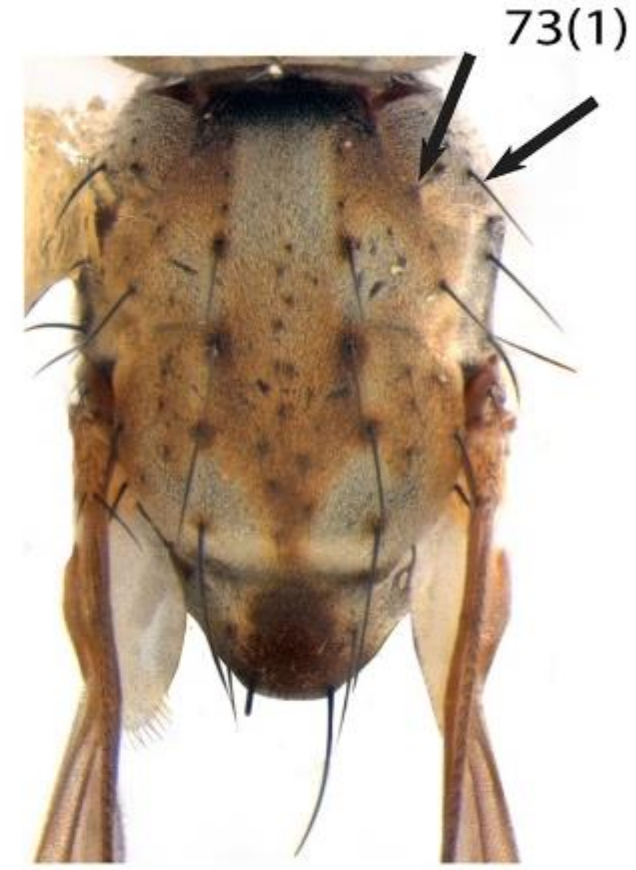

(b)

Figure 71a-b. Character 73, postpronotal seta: a. Calamoncosis minima, state 0 , zero or one; $b$. Siphonellopsis lacteibasis, state 1 , two or more. 


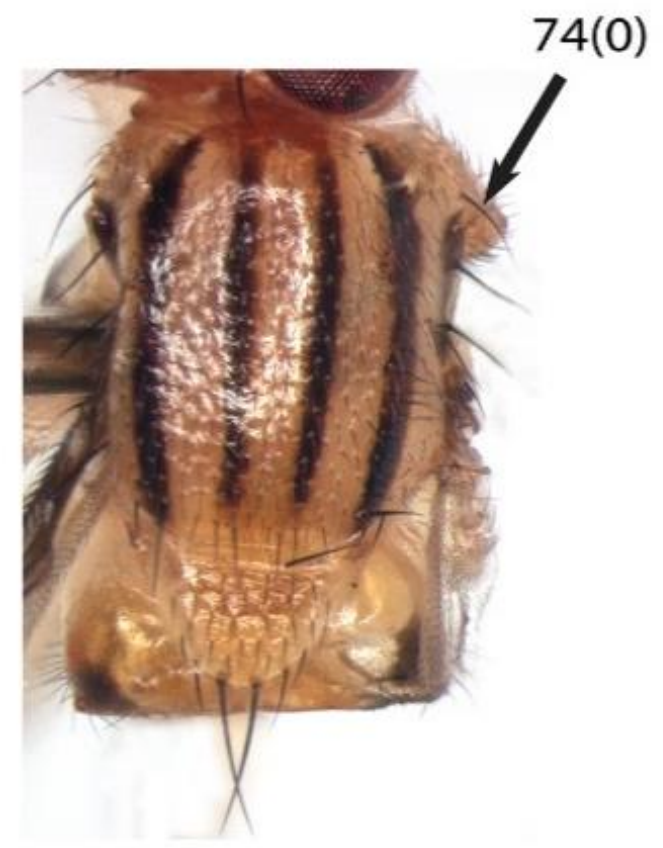

(a)

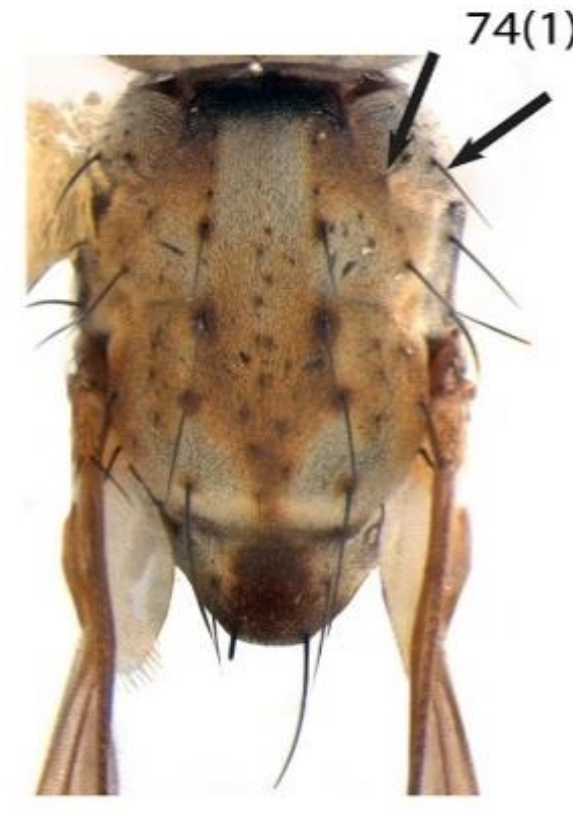

(b)

Figure 72a-b. Character 74, postpronotal seta orientation, in case of 2 or more setae: a. Metasiphonella sp., state 0 , only lateroclinate; b. Siphonellopsis lacteibasis, state 1 , reclinate and lateroclinate.

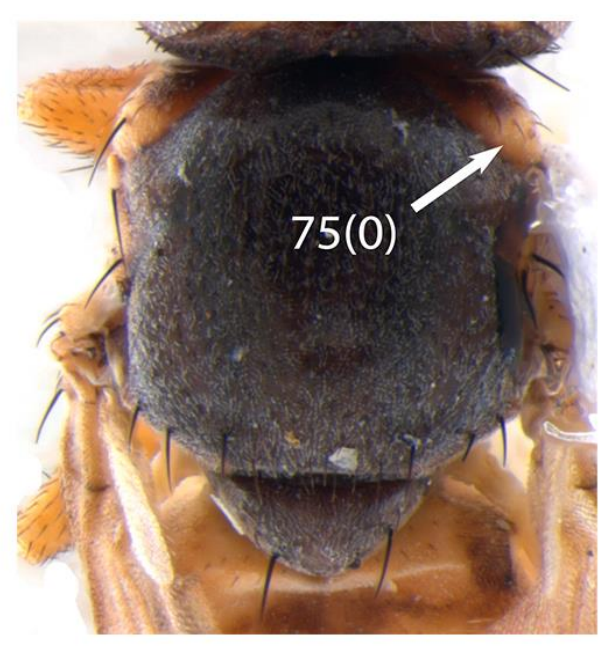

(a)

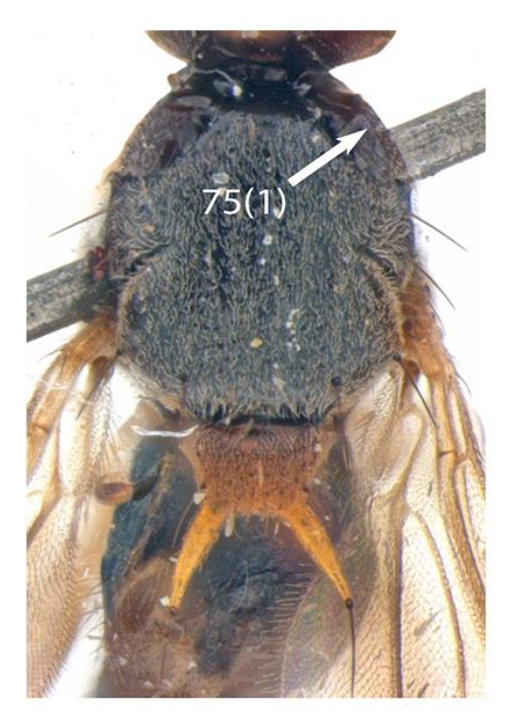

(b)

Figure 73a-b. Character 75, postpronotal lobe: a. Dicraeus vagans, state 0 , mostly yellow; $b$. Disciphus peregrinus, state 1 , mostly brown or black. 


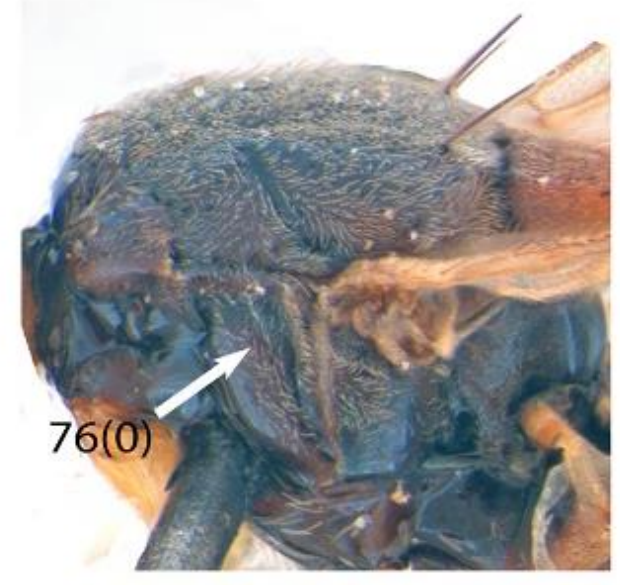

(a)

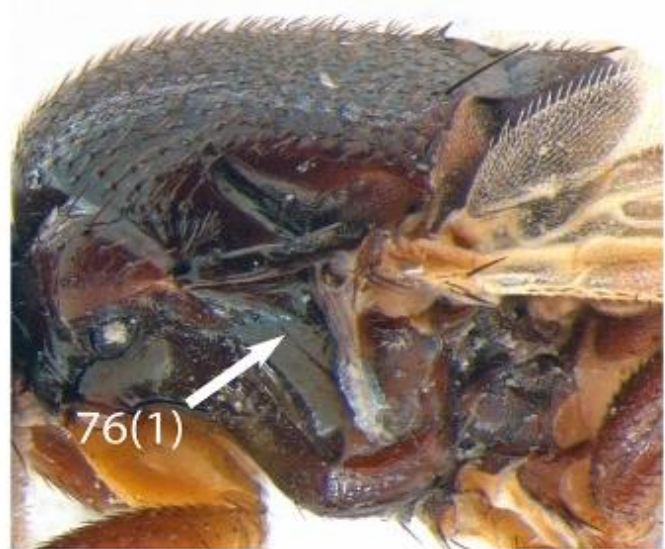

(b)

Figure 74a-b. Character 76, anepisternum: a. Disciphus peregrinus, state 0, purinosity; b. Epimadiza sp, state 1 , bare.

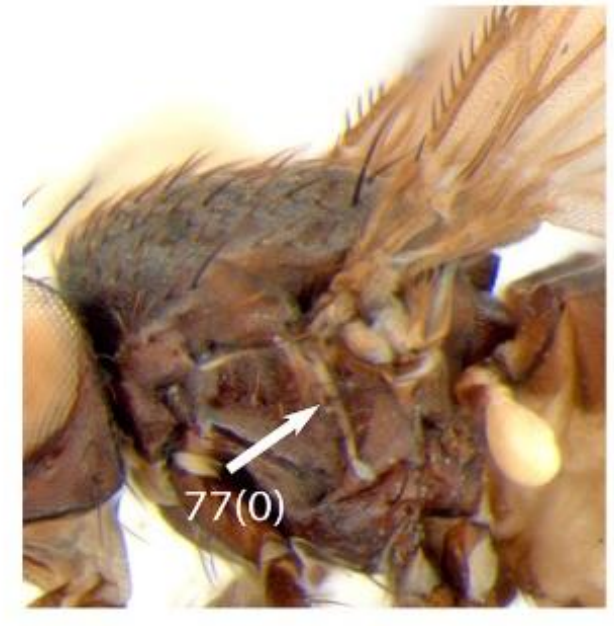

(a)

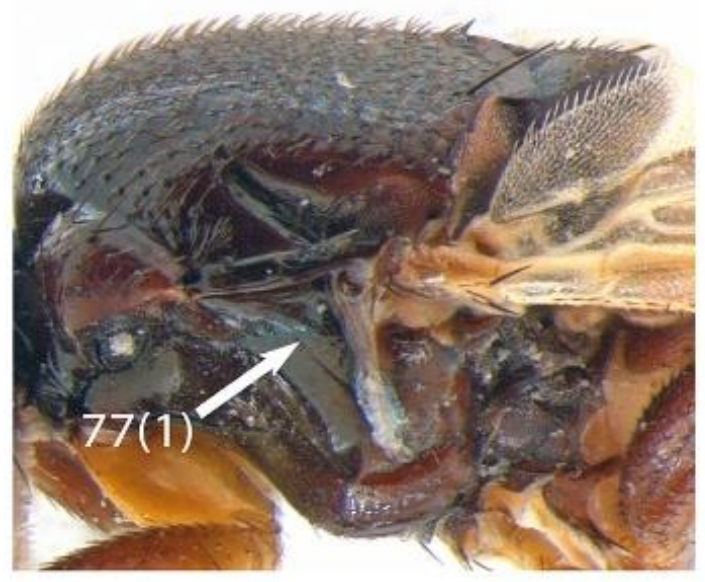

(b)

Figure 75a-b. Character 77, anepisternum setaceous: a. Meoneura flavifacies, state 0 , present; b. Epimadiza sp., state 1, absent. 


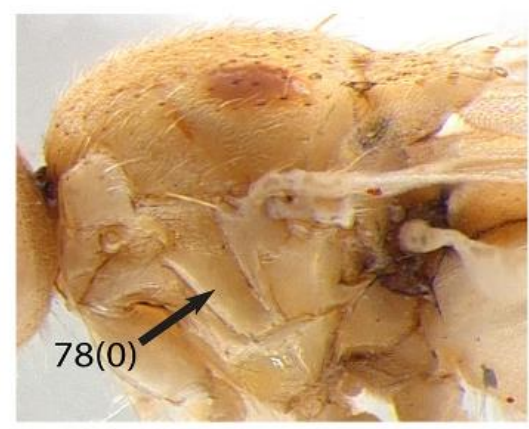

(a)

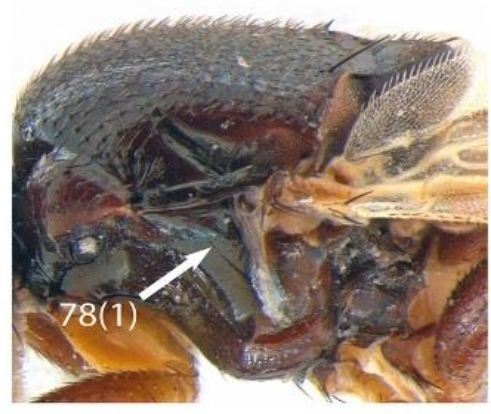

(b)

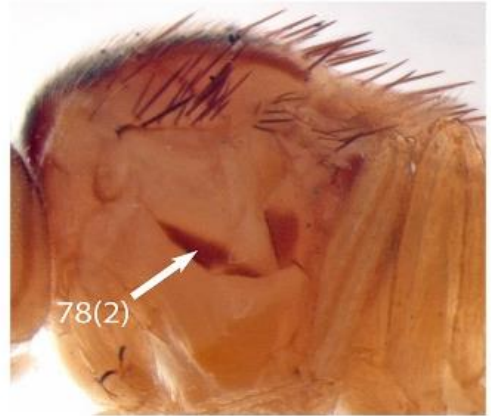

(c)

Figure 76a-c. Character 78, anepisternum color: a. Elachiptera bimaculata, state 0 , entirely yellow(ish); b. Epimadiza sp., state 1, entirely brown or black; c. Eugaurax sp., state 2, mostly yellow with brown marketing ventrally.

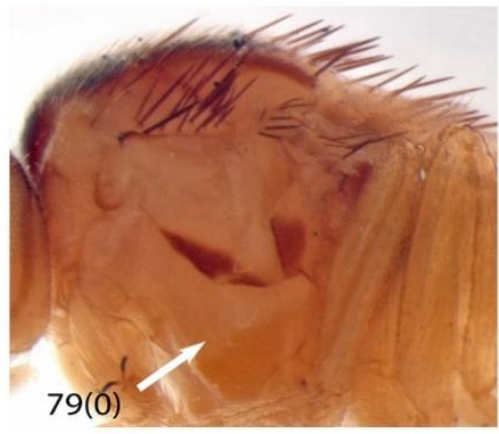

(a)

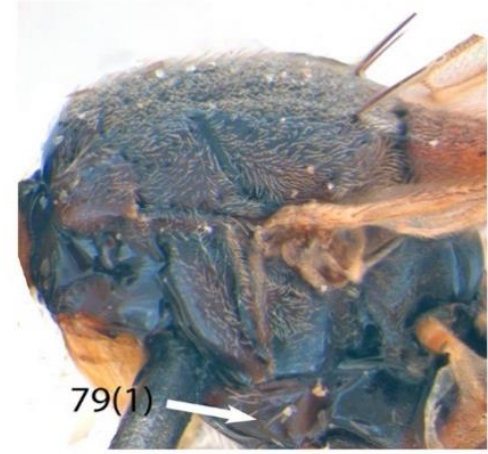

(b)

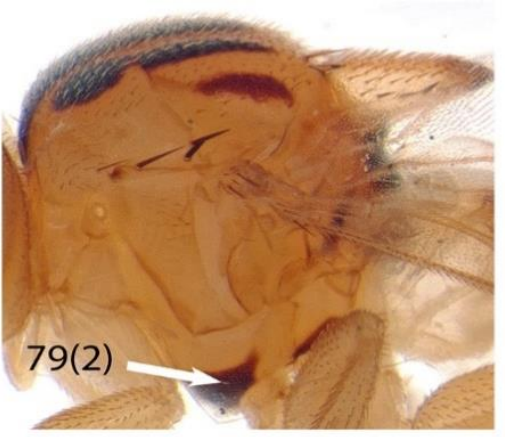

(c)

Figure 77a-c. Character 79, katepisternum color: a. Eugaurax sp., state 0, entirely yellow(ish); b. Disciphus peregrinus, state 1, entirely brown or black; c. Paracalamoncosis brasiliensis (Photography by Paula Raile Riccardi), state 2, mostly yellow with brown marketing. 


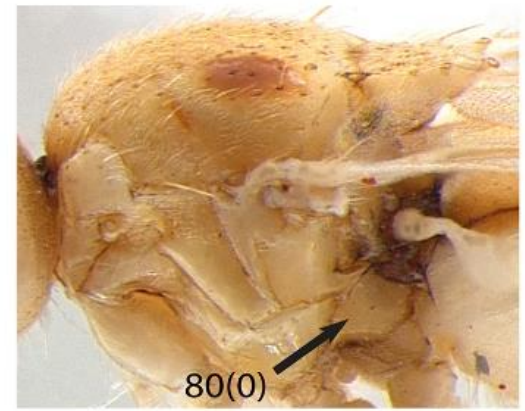

(a)

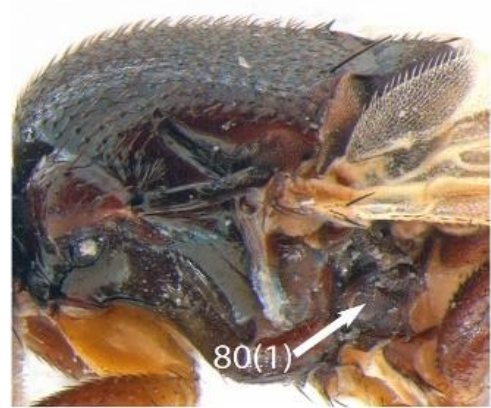

(b)

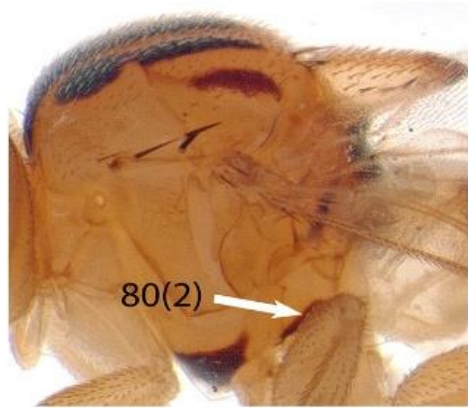

(c)

Figure 78a-c. Character 80, meron color: a. Elachiptera bimaculata, state 0 , yellow or light brown; b. Epimadiza sp., state 1, brown or black; c. Paracalamoncosis brasiliensis (Photography by Paula Raile Riccardi), state 2, yellow(ish), dark marketing.

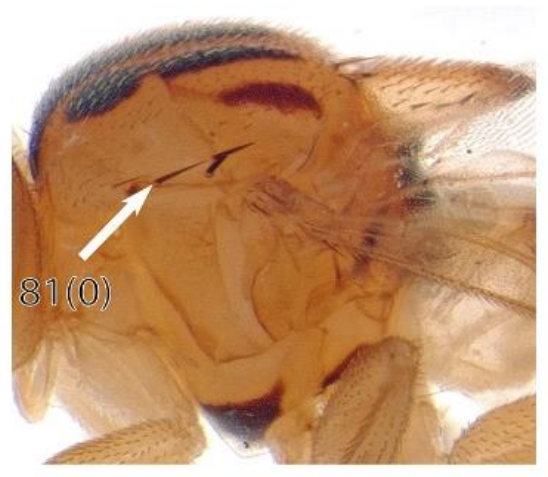

(a)

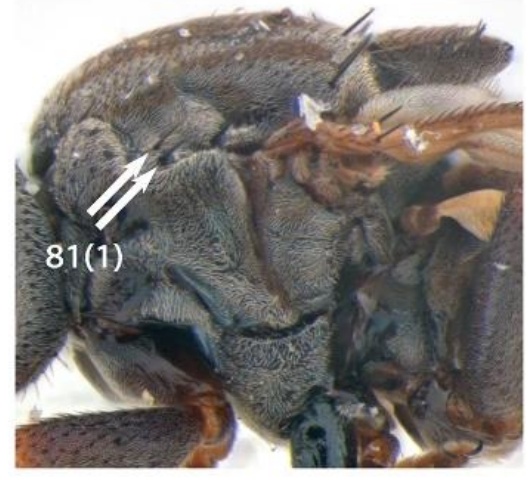

(b)

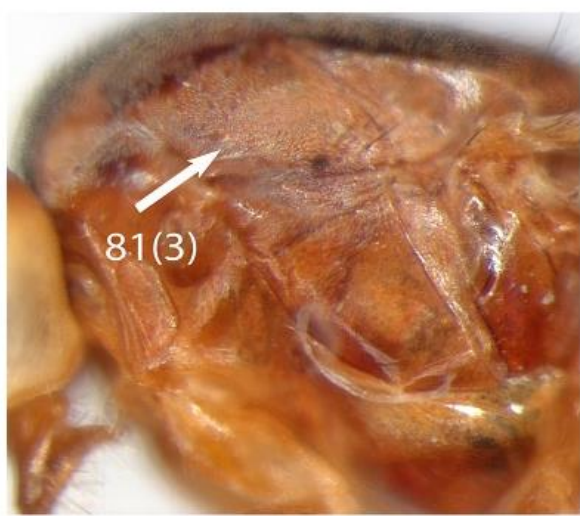

(d)

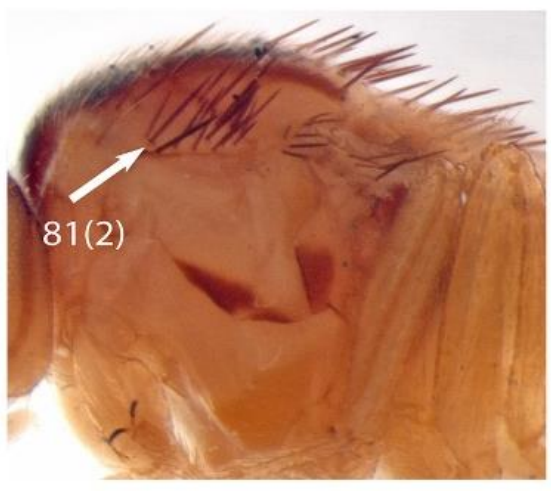

(c)

Figure 79a-d. Character 81, number of distinct anterior notopleural setae: a. Paracalamoncosis brasiliensis (Photography by Paula Raile Riccardi), state 0 , one; b. Aphanotrigonum trilineatus, state 1, two; c. Eugaurax sp., state 2, more; d. Ectecephala albistylum (Photography by Paula Raile Riccardi), state 3 , zero. 


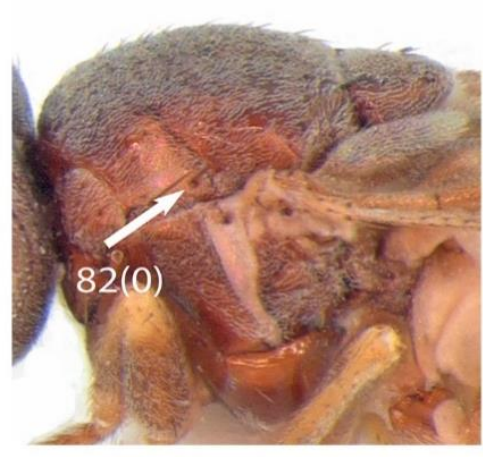

(a)

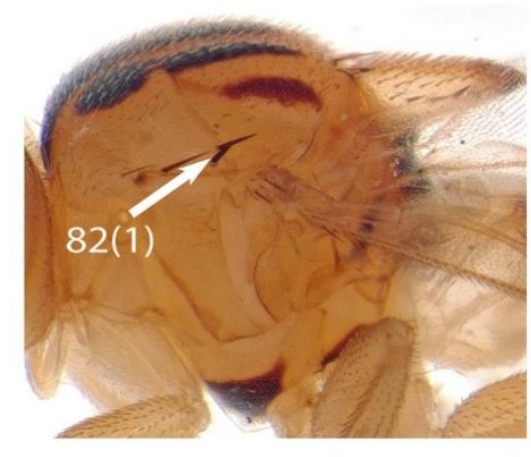

(b)

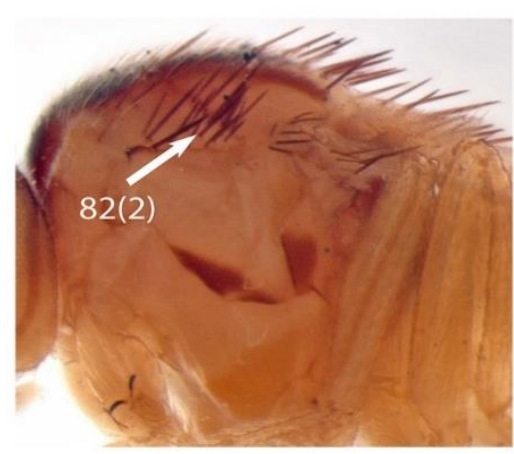

(c)

Figure 80a-c. Character 82, number of distinct posterior notopleural setae: a. Microcercis albipalpis, state 0, one; b. Paracalamoncosis brasiliensis (Photography by Paula Raile Riccardi), state 1, two; c. Eugaurax sp., state 2, more.

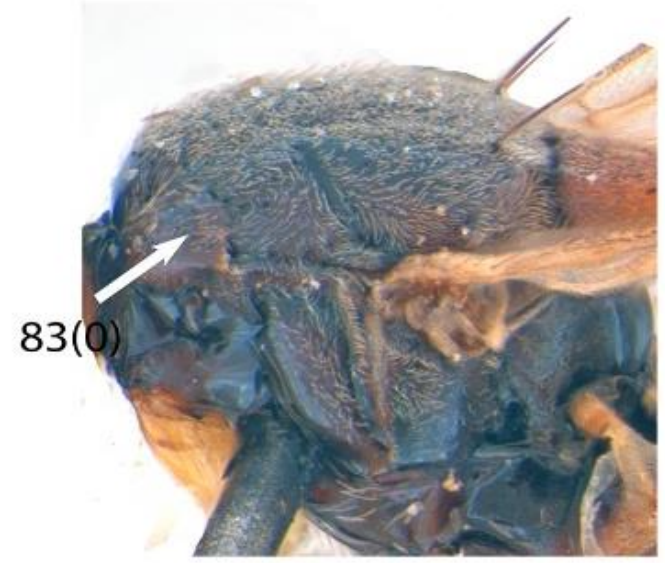

(a)

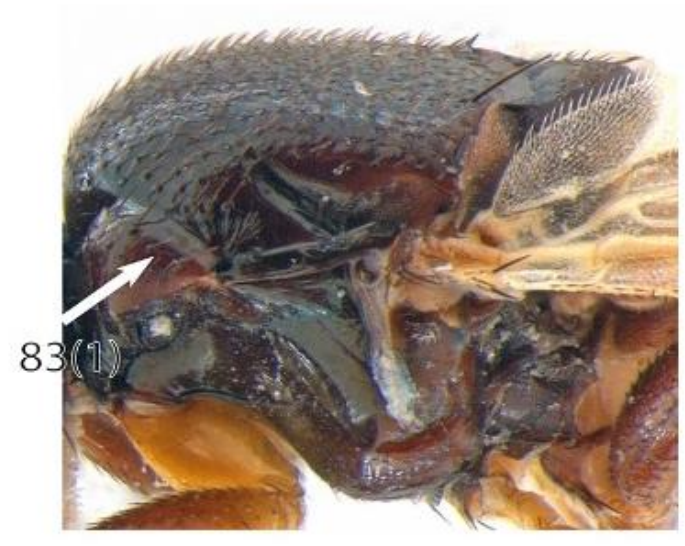

(b)

Figure 81a-b. Character 83, purinosity on postpronotal lobe: a. Disciphus peregrinus, state 0, present; b. Epimadiza sp., state 1, absent. 


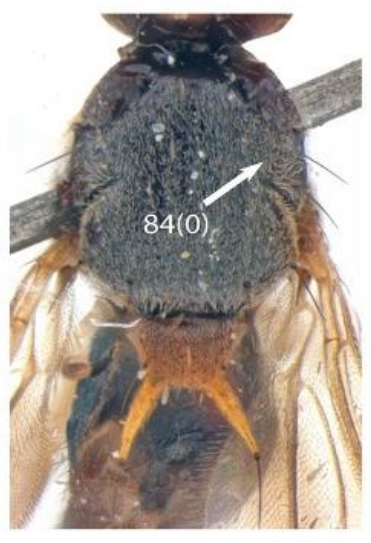

(a)

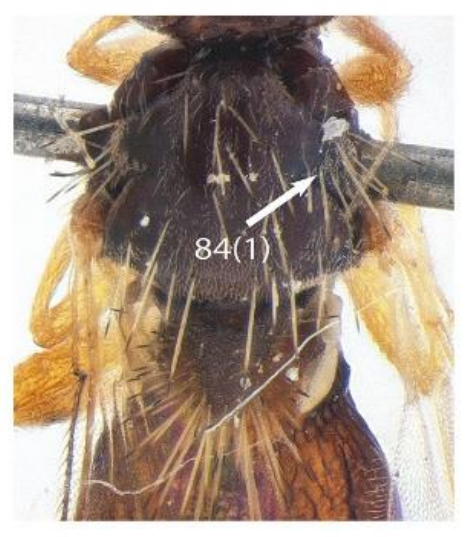

(b)

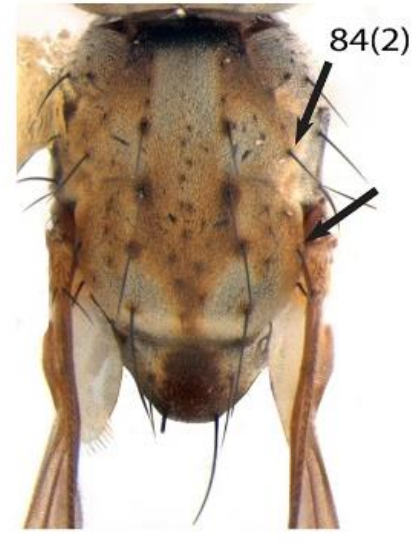

(c)

Figure 82a-c. Character 84, numbers of supraalars: a. Disciphus peregrinus, state 0 , zero; b. Anatrichus erinaceus, state 1 , one; c. Siphonellopsis lacteibasis, state 2, two.

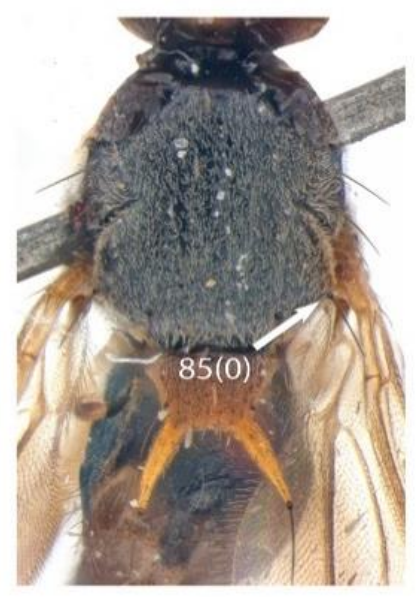

(a)

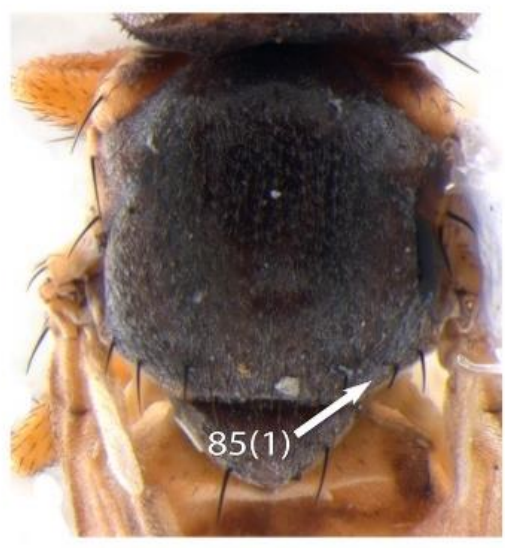

(b)

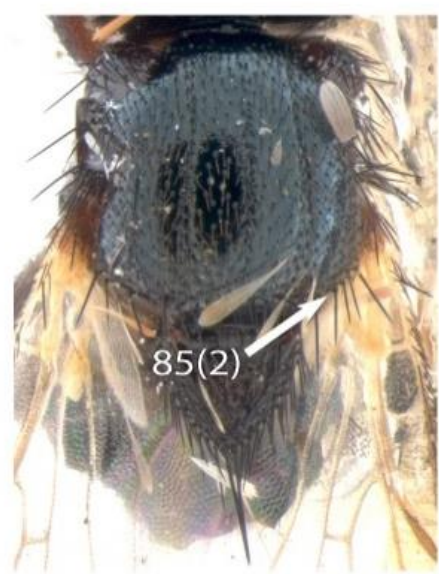

(c)

Figure 83a-c. Character 85, numbers of postalars: a. Disciphus peregrinus, state 0 , one; b. Dicraeus vagans, state 1, two; c. Chaetochlorops inquilina, state 2, more. 


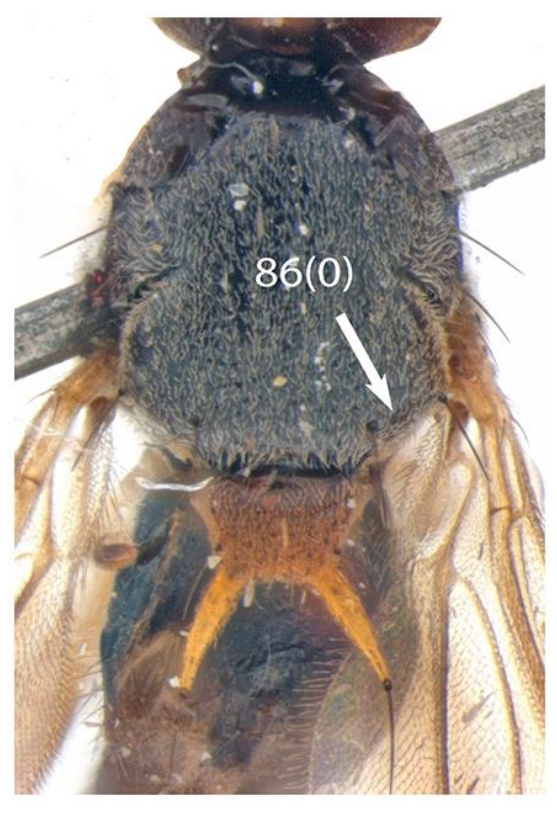

(a)

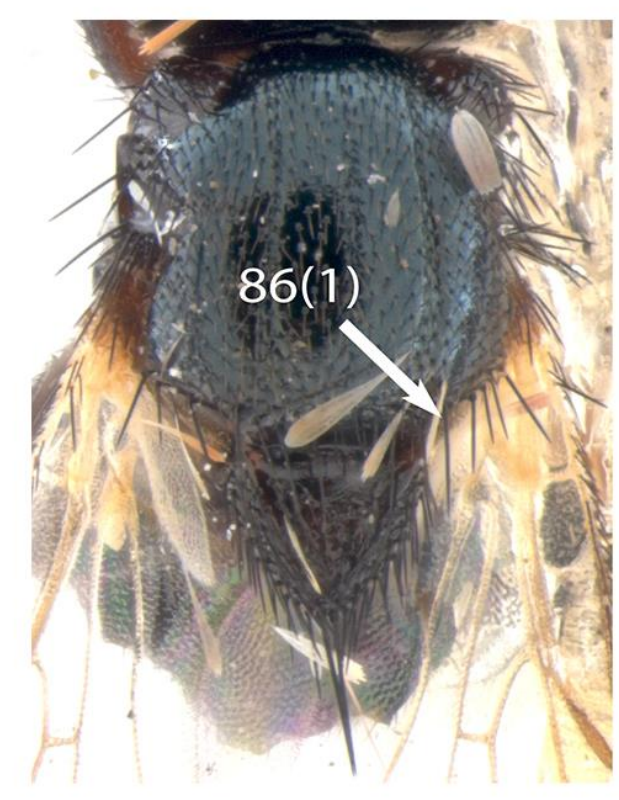

(b)

Figure 84a-b. Character 86, intrapostalar: a. Disciphus peregrinus, state 0 , absent; $b$. Chaetochlorops inquilina, state 1 , present.

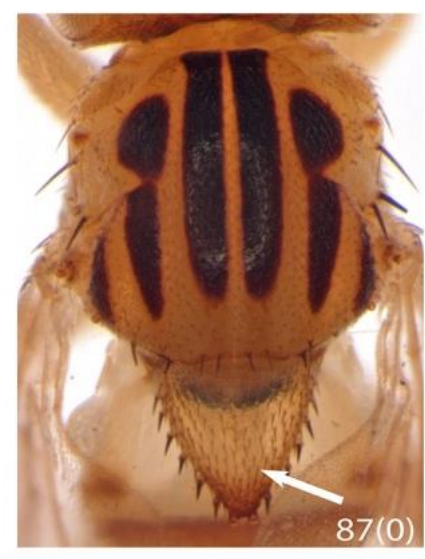

(a)

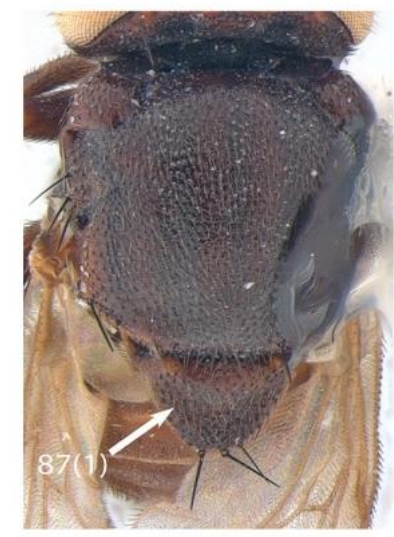

(b)

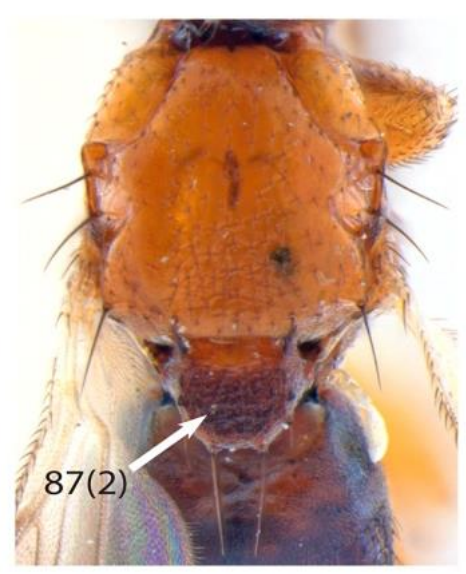

(c)

Figure 85a-c. Character 87, length of scutellum: a. Paracalamoncosis brasiliensis (Photography by Paula Raile Riccardi), state 0, longer than wide; b. Calamoncosis minima, state 1, wider than long; c. Elachiptera brevipennis, state 2, almost as long as wide. 


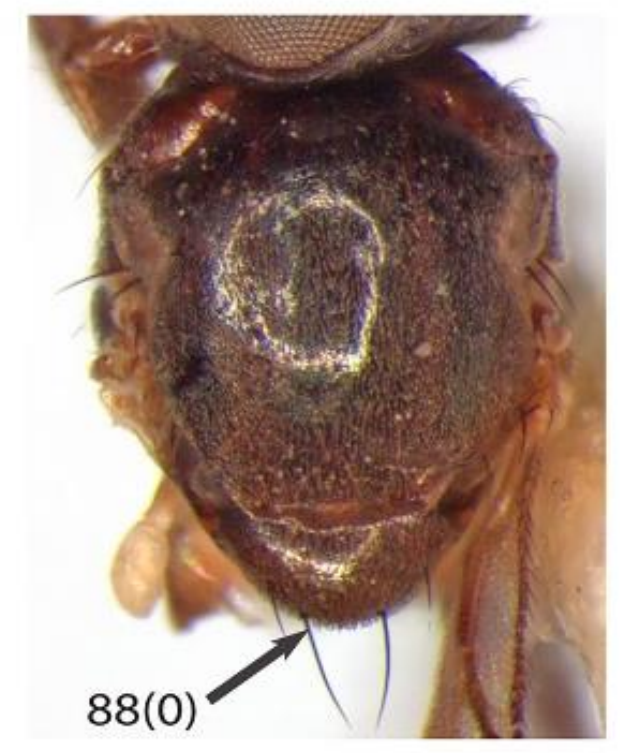

(a)

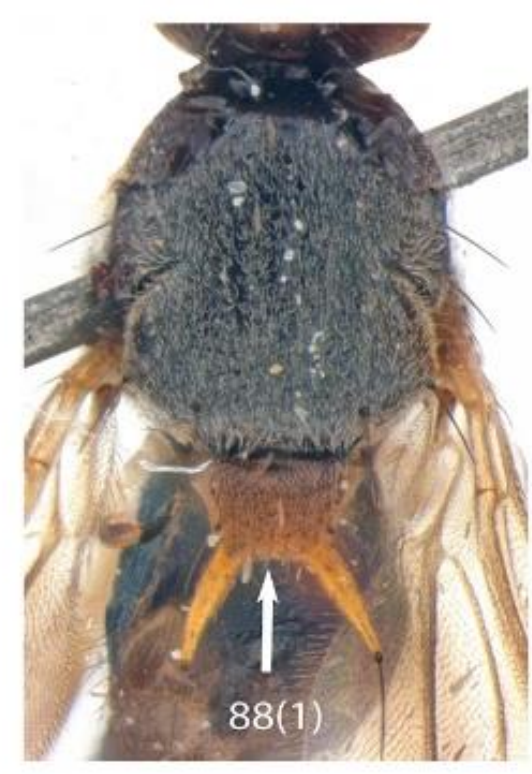

(b)

Figure 86a-b. Character 88, tip of scutellum in dorsal view: a. Apallates dissidens, state 0 , apically rounded or slightly pointed; b. Disciphus peregrinus, state 1 , apically straight (flattened).

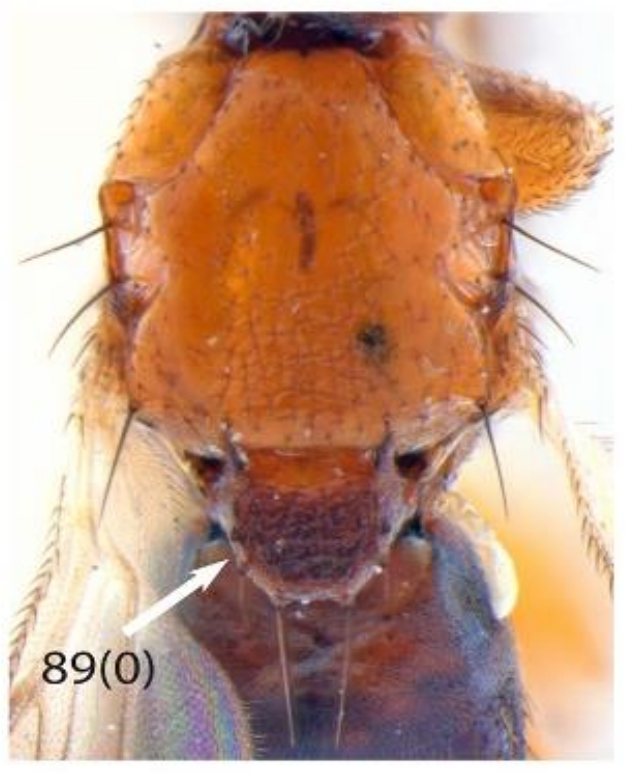

(a)

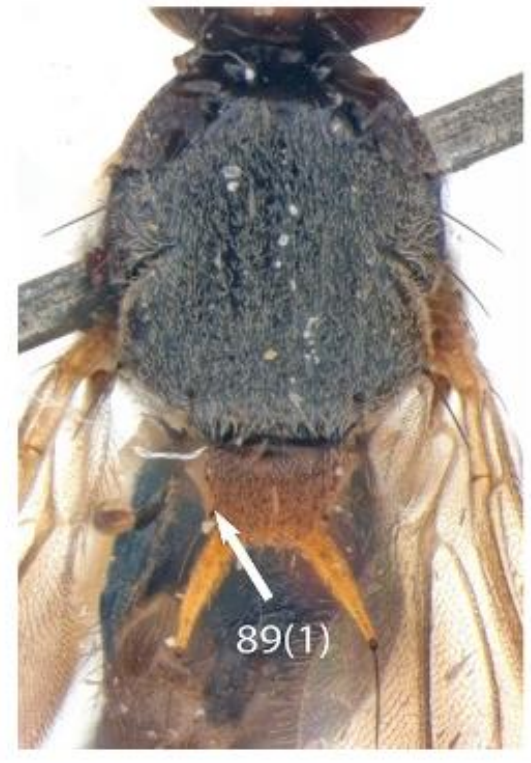

(b)

Figure 87a-b. Character 89, laterals of scutellum in dorsal view: a. Elachiptera brevipennis, state 0 , convex; b. Disciphus peregrinus, state 1 , straight. 


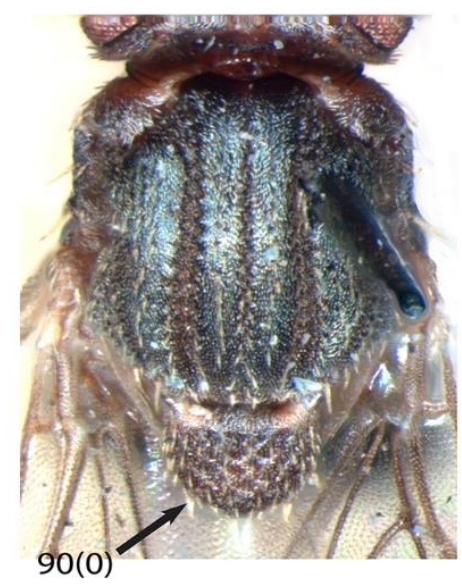

(a)

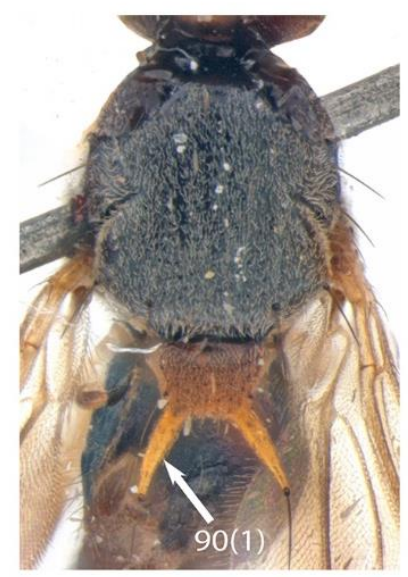

(b)

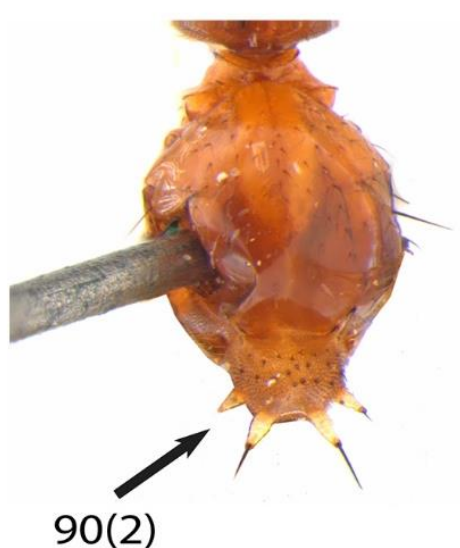

(c)

Figure 88a-c. Character 90, scutellar finger-like tubercles: a. Tricimba lineella, state 0, absent; b. Disciphus peregrinus, state 1, present, only apicals; c. Sepsidoscinis maculipennis, state 2, present, apicals and subapicals.

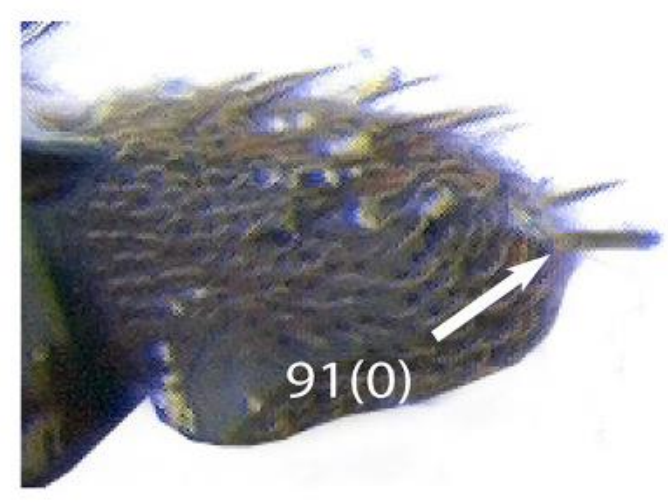

(a)

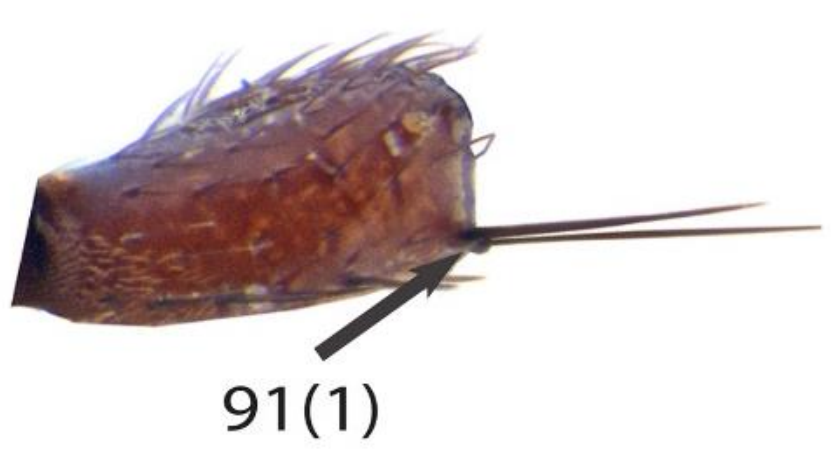

(b)

Figure 89a-b. Character 91, scutellar large setae position: a. Hapleginella laevifrons, state 0 , marginal; b. Heteroscinis hystrix, state 1 , ventral. 


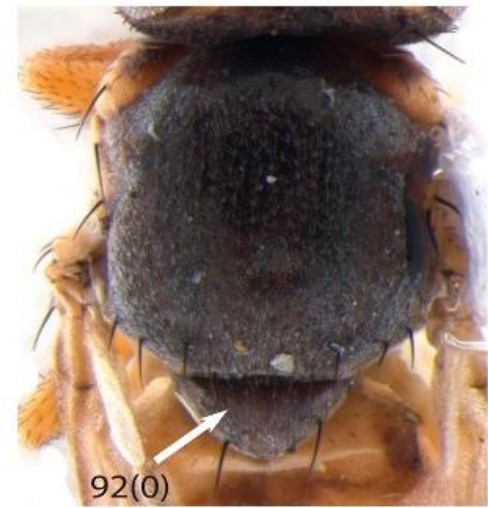

(a)

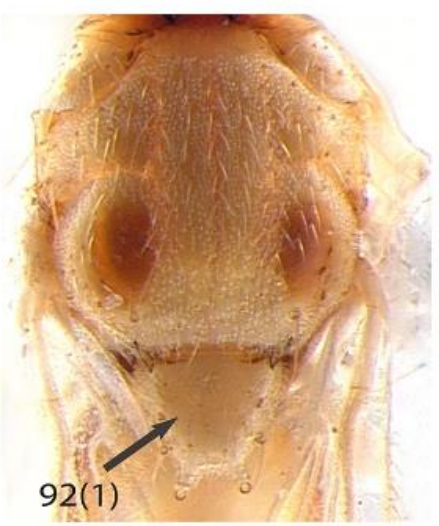

(b)

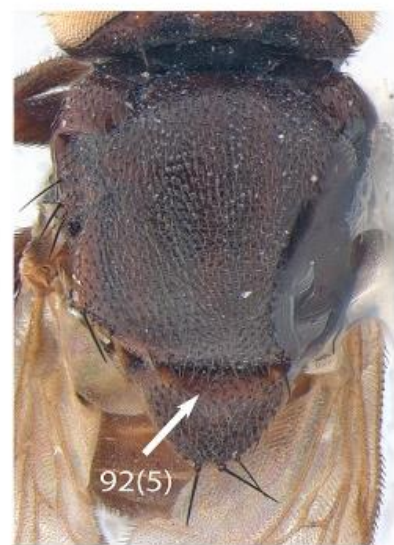

(d)

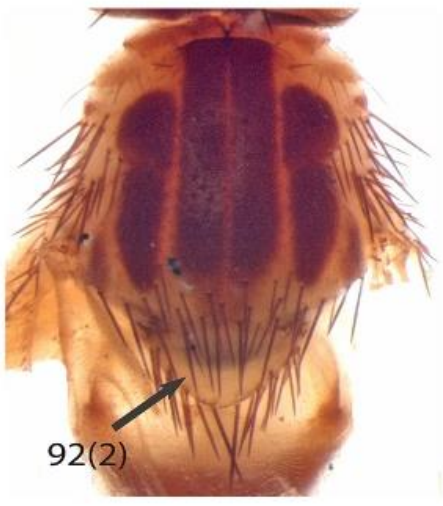

(c) black; b. Elachiptera bimaculata, state 1, entirely yellow or light brown; c. Eugaurax sp., state 2, apically and laterally yellow or brown; d. Calamoncosis minima, state 5 , basally yellow or brown. 


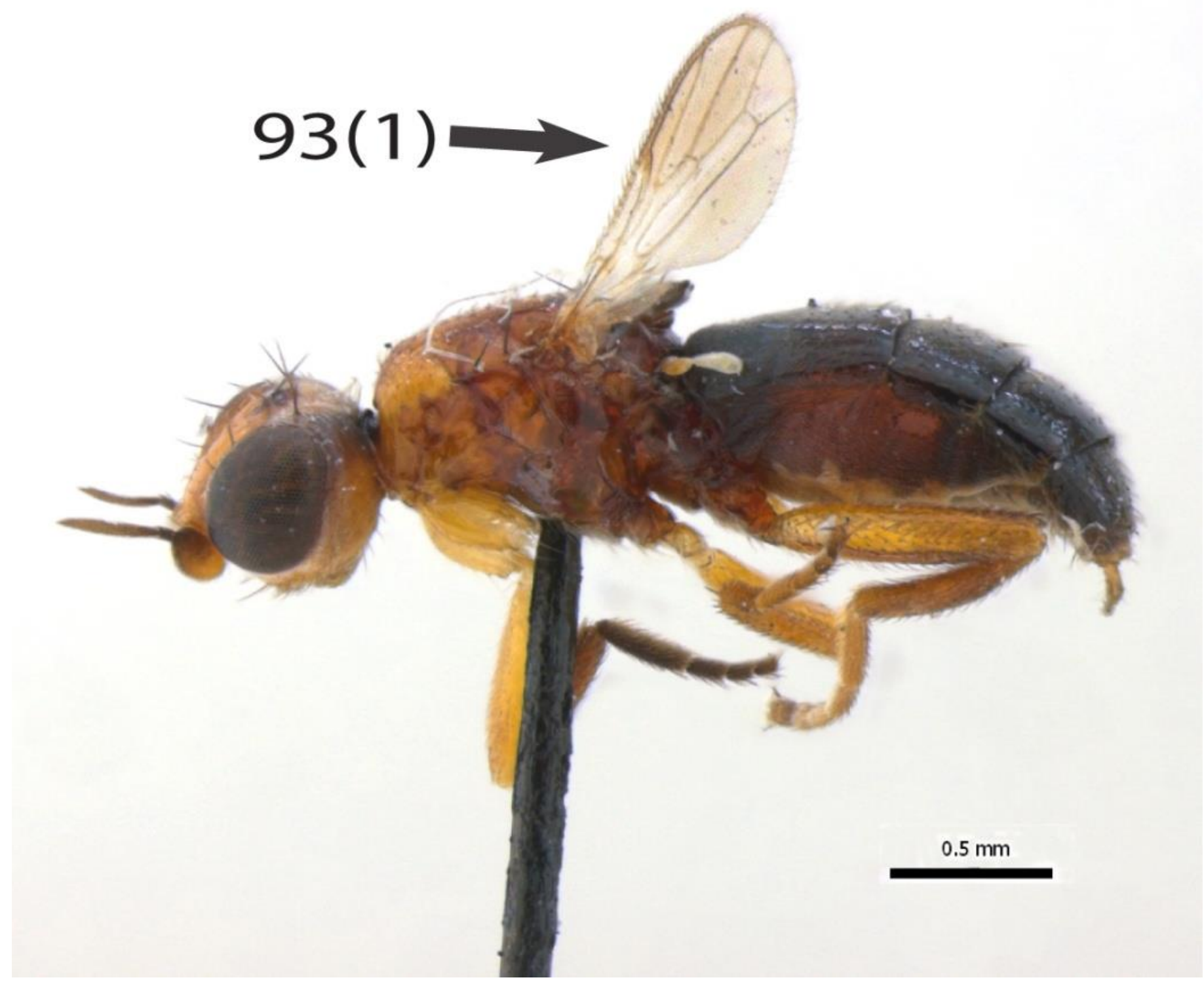

Figure 91: Character 93, wing length compared to abdomen length: Elachiptera brevipennis, state 1, shorter. 


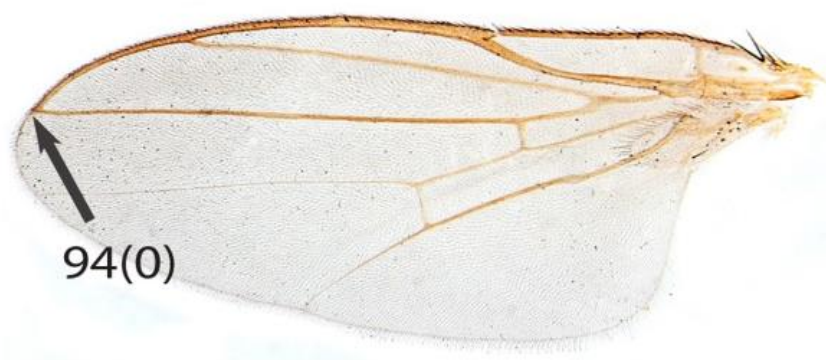

(a)

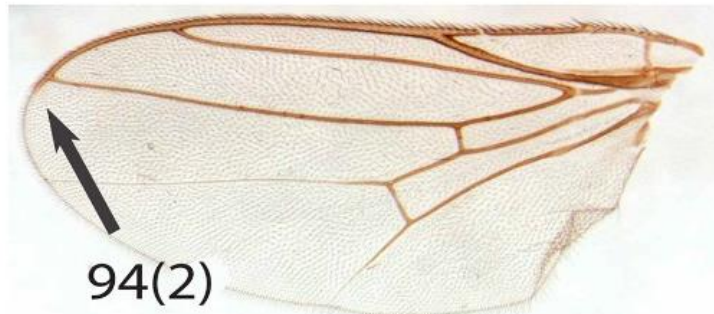

(c)

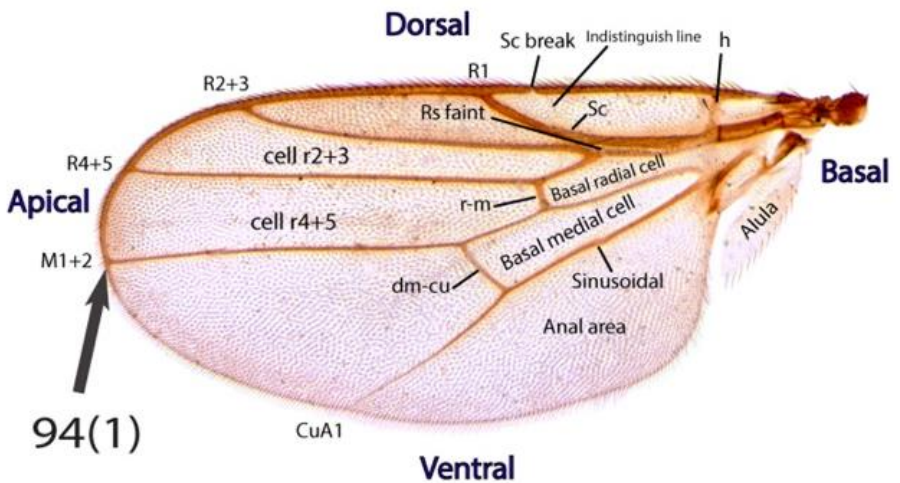

(b)

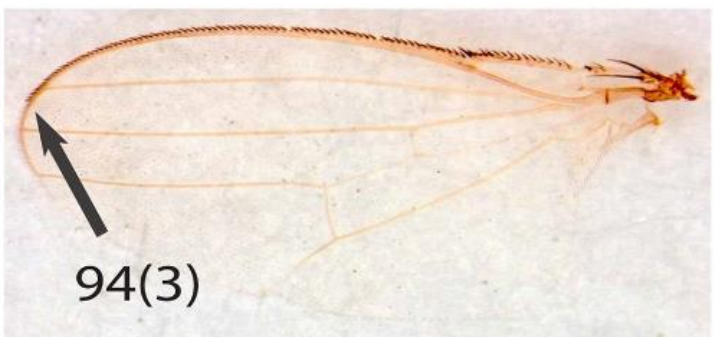

(d)

Figure 92a-d. Character 94, costal: a. Chlorops pumilionis (Photography by Paula Raile Riccardi), state 0 , ending at $R_{4+5} ;$ b. Siphonella oscinina (with areas of the wing), state 1 , ending at $\mathrm{M}_{1+2} ; \mathrm{c}$. Hapleginella laevifrons, state 2 , ending between $\mathrm{R}_{4+5}$ and $\mathrm{M}_{1+2}$; $\mathrm{d}$. Heteroscinis hystrix, state 3 , ending before $\mathrm{R}_{4+5}$.

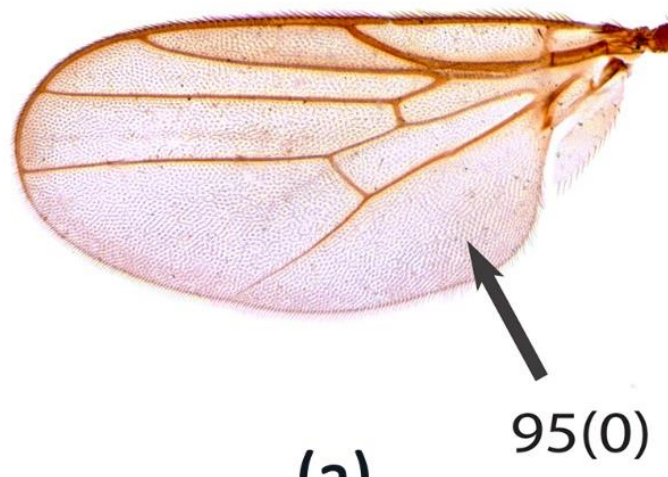

(a)

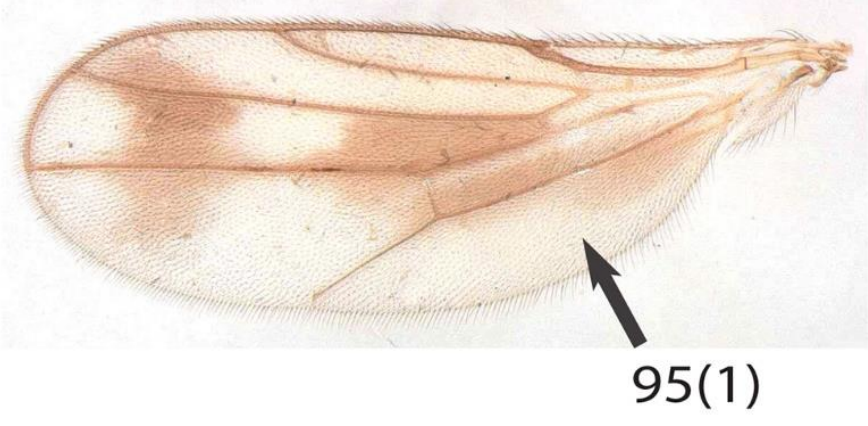

(b)

Figure 93a-b. Character 95, anal lobe: a. Siphonella oscinina, state 0, present; b. Disciphus peregrinus, state 1 , absent. 


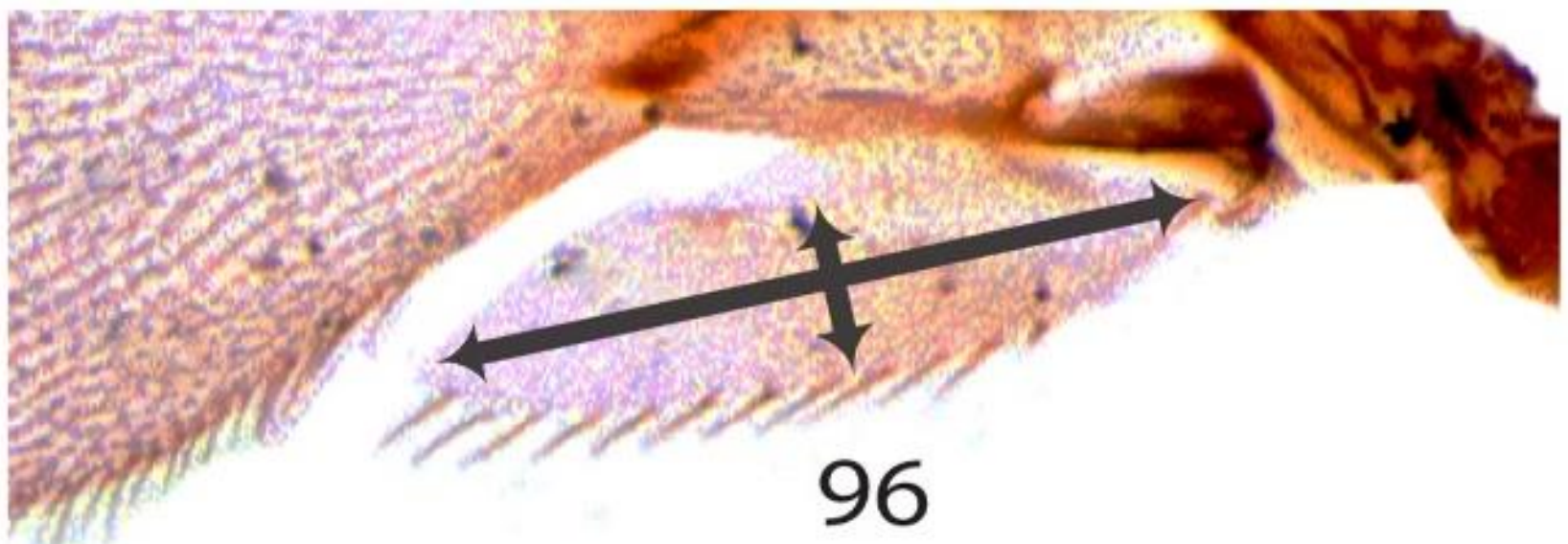

Figure 94: Character 96, alula (length/width): Siphonella oscinina.

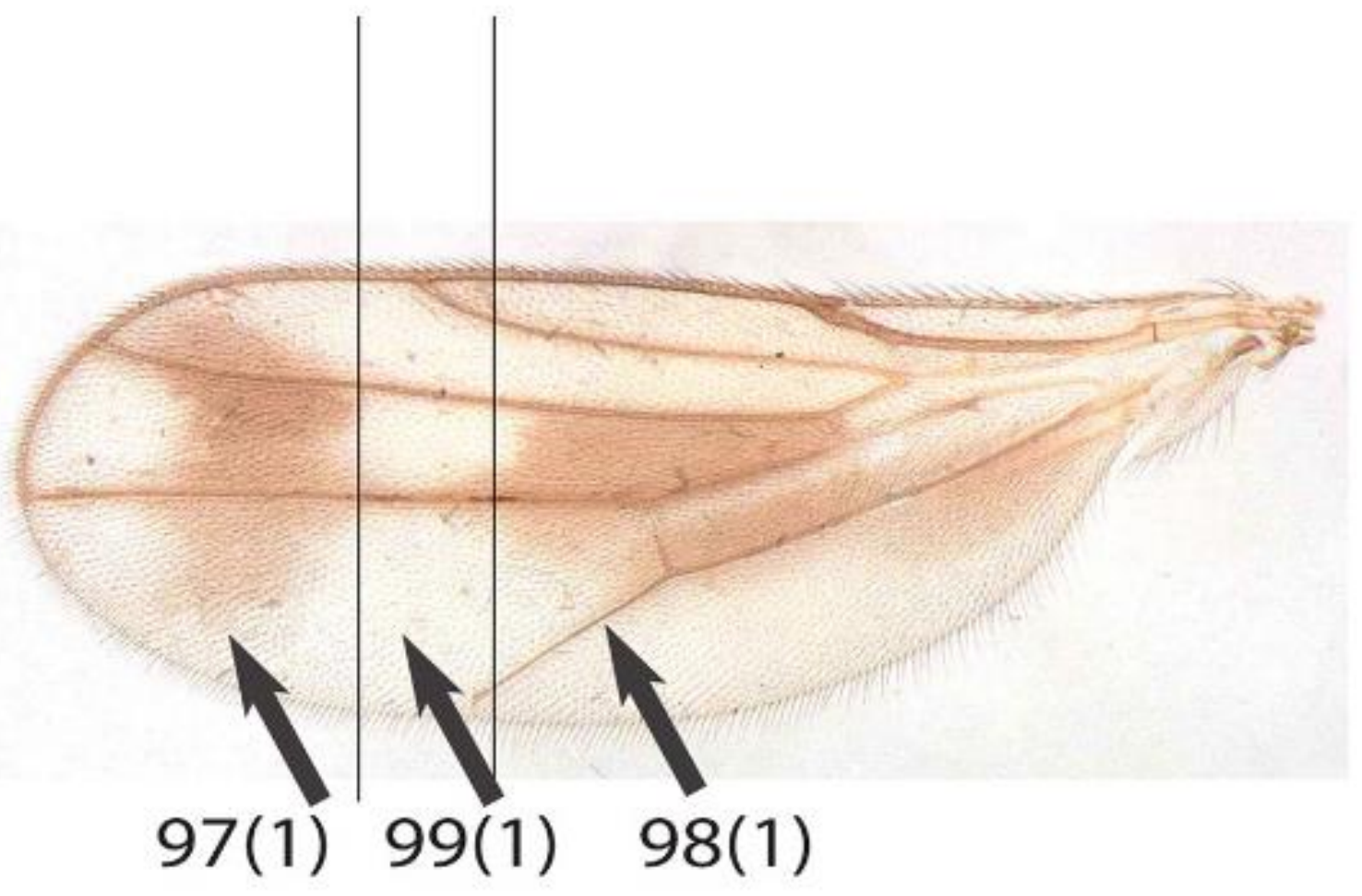

Figure 95: Characters 97-98; C97, pattern on the wing, apically; C98, pattern on the wing, basally; C99, pattern on the wing, medially: Disciphus peregrinus, C97, state 1, marked; C98, state 1 , marked; C99, state 1, marked. 


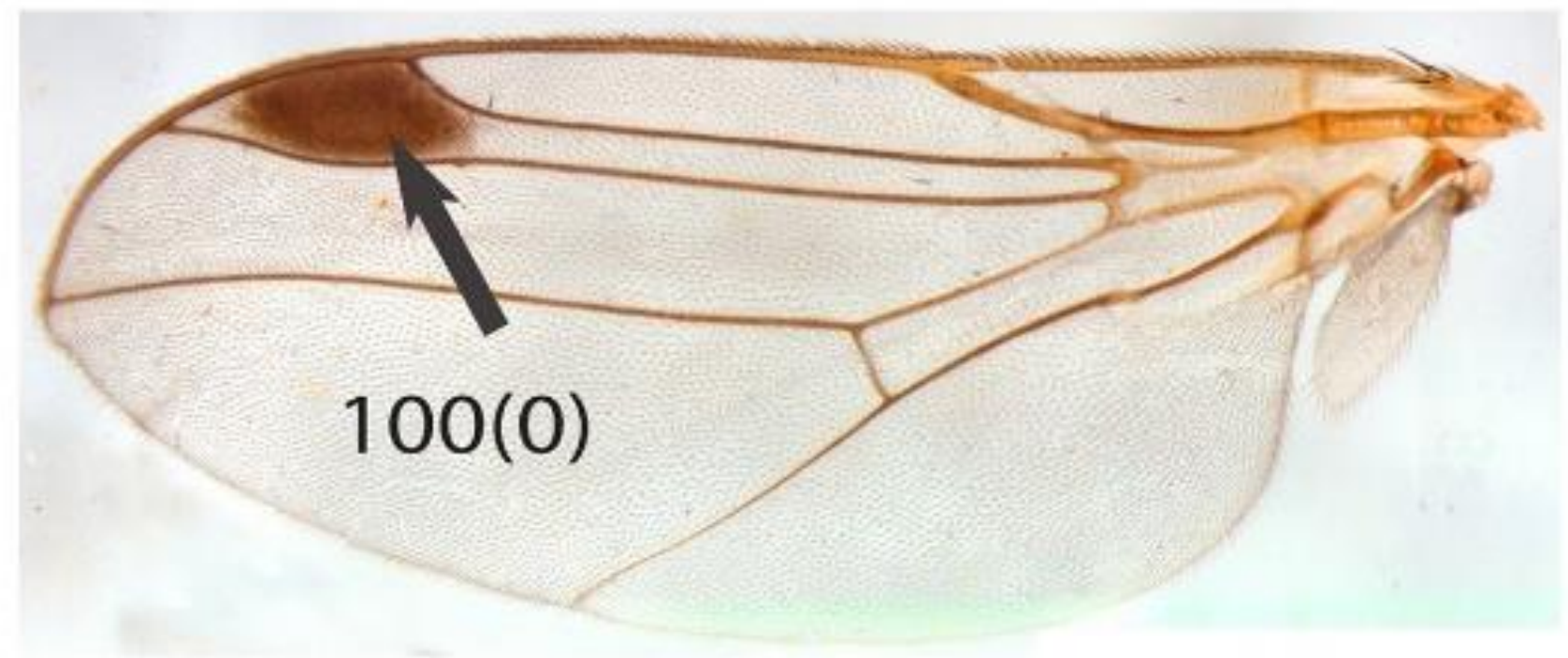

Figure 96: Character 100, shape of pattern on the wing: Daltastoma unipunctatum, state 0 , restricted between veins.

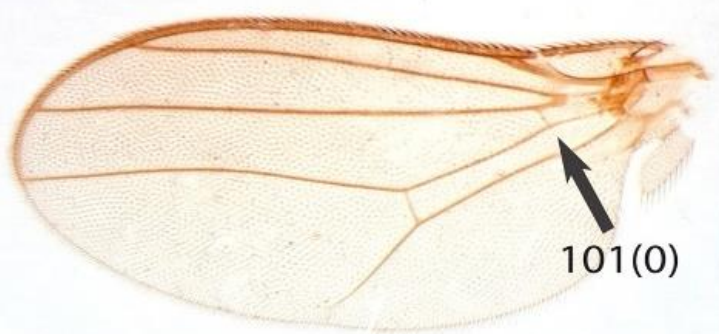

(a)

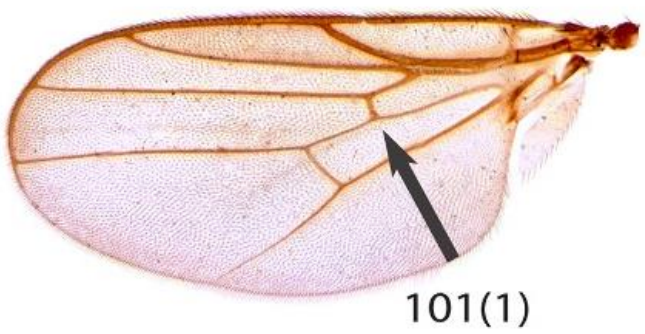

(b)

Figure 97a-b. Character 101, basal radial cell relating to the basal medial cell: a. Notaulacella sp., state 0 , reaches before medium of basal medial cell; $b$. Siphonella oscinina, state 1 , at least at the medium of basal medial cell. 


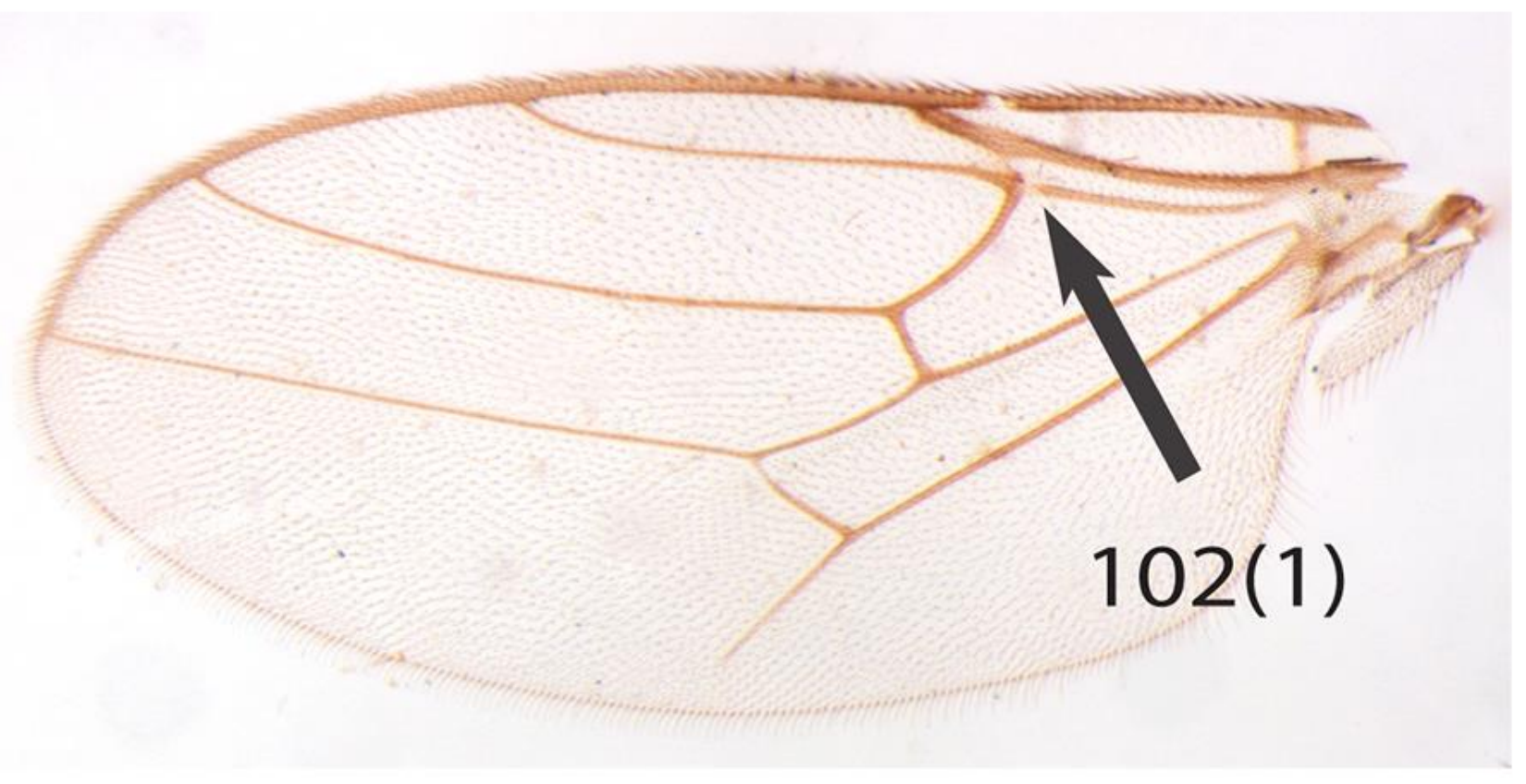

Figure 98: Character 102, height of basal radial cell: Loxobathmis obliquans, state 1, projecting dorsally.

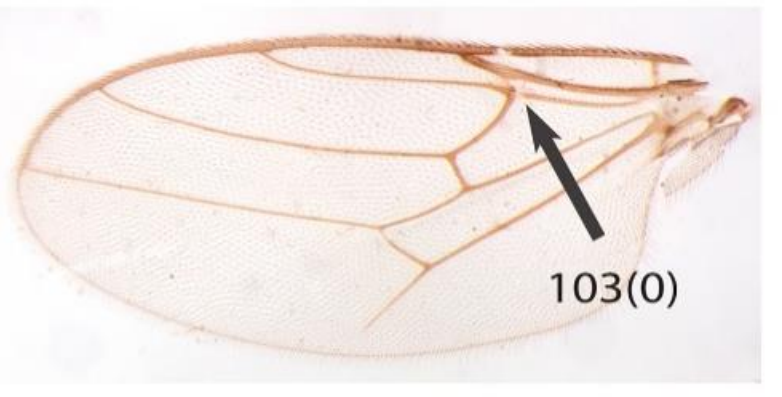

(a)

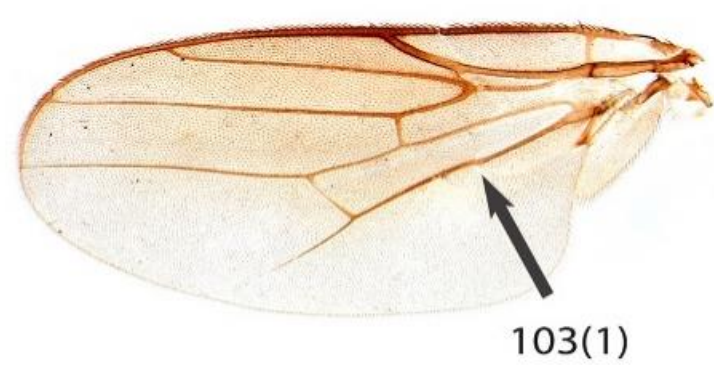

(b)

Figure 99a-b. Character 103, sinusoidal of CuA1 (flexure part): a. Loxobathmis obliquans, state 0, absent (straight or slightly inward); b. Lasiambia palposa, state 1, present (notably inward). 


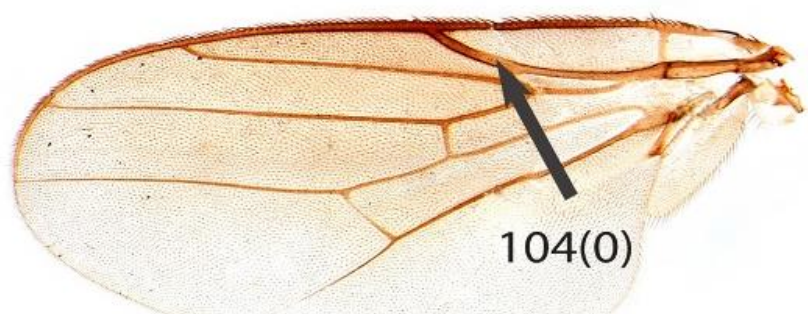

(a)

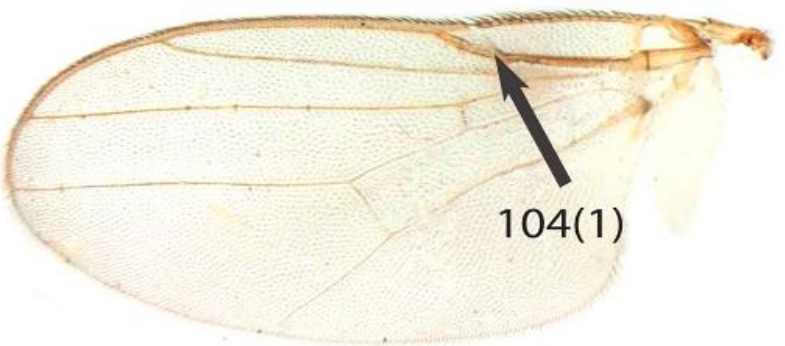

(b)

Figure 100a-b. Character 104, $\mathrm{R}_{1}$ : a. Lasiambia palposa, state 0, slightly distally; b. Hippelates plebejus, state 1 , with a short bump.

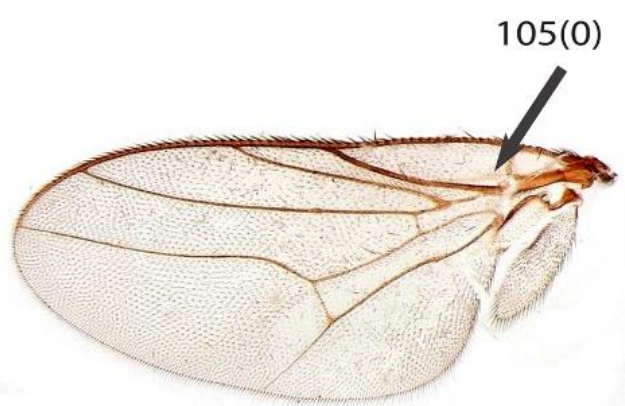

(a)

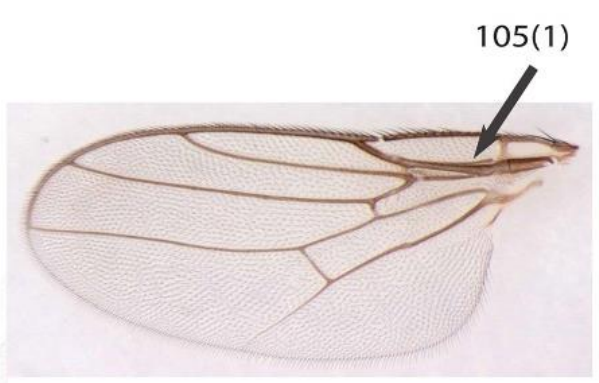

(b)

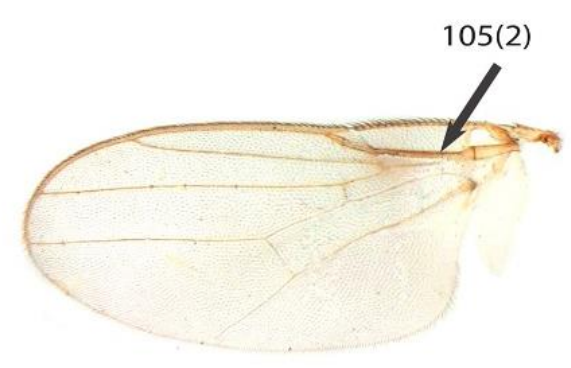

(c)

Figure 101a-c. Character 105, Sc: a. Rhodesiella sp.2, state 0, almost entirely independent from $\mathrm{R}_{1}$; b. Metasiphonella sp., state 1 , distinct from $\mathrm{R}_{1}$ basally; c. Hippelates plebejus, state 2, entirely indistinctive.

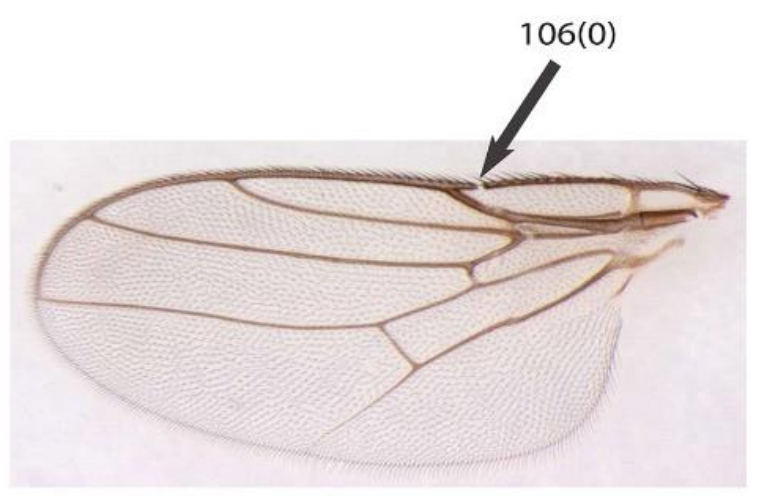

(a)

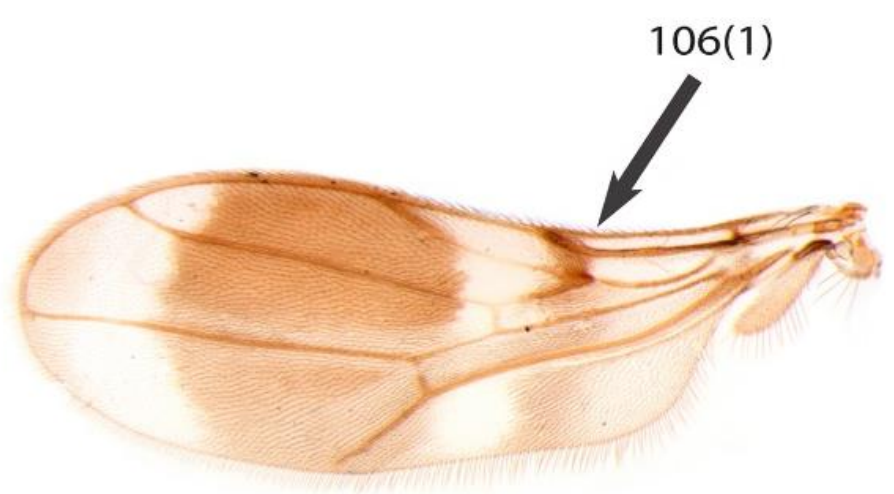

(b)

Figure 102a-b. Character 106, costal break on Sc part: a. Metasiphonella sp., state 0, present; b. Sepsidoscinis maculipennis, state 1 , absent. 


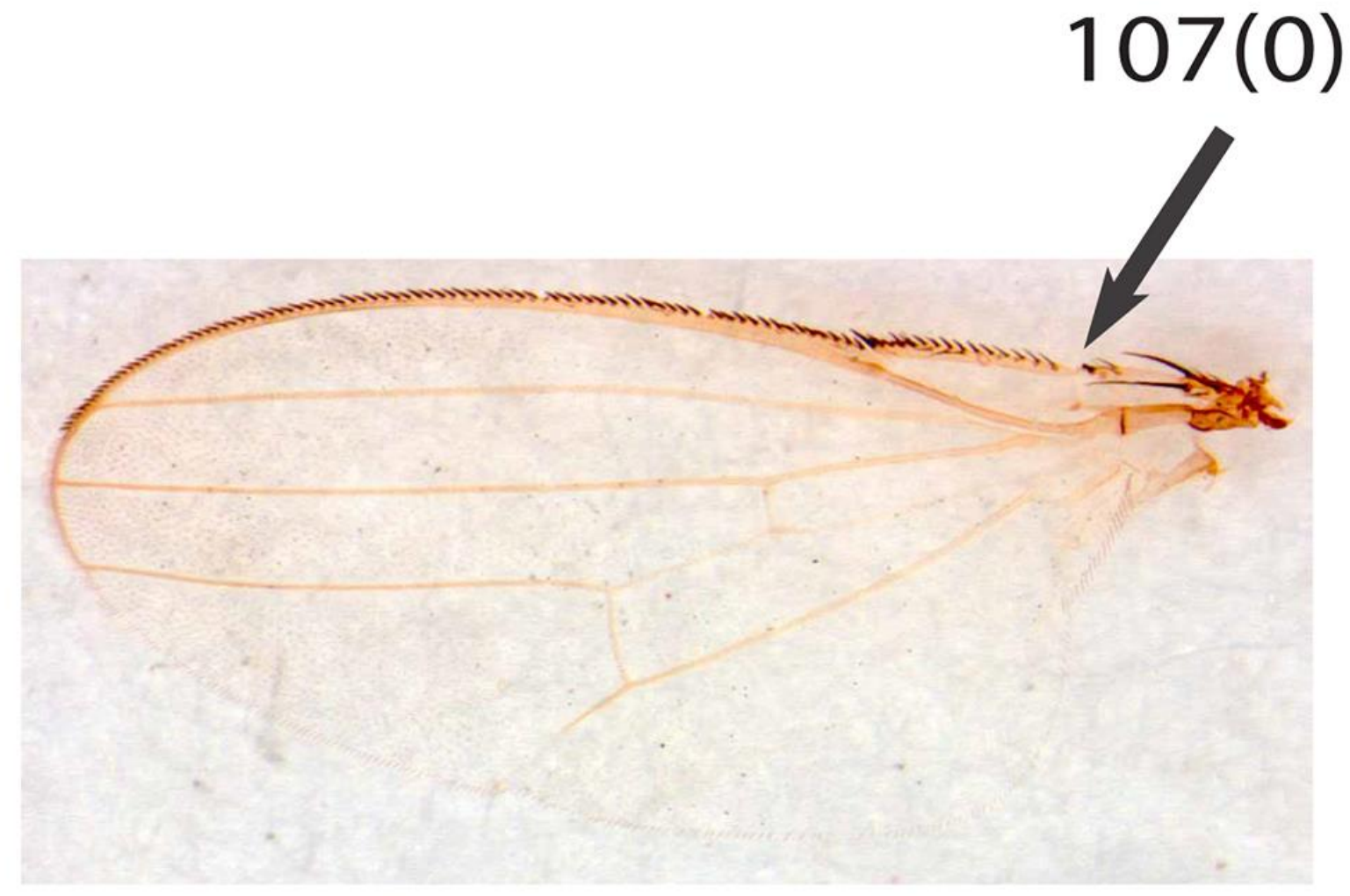

Figure 103: Character 107, costal break on humeral part of wing: Madiza glabra, state 0 , present.

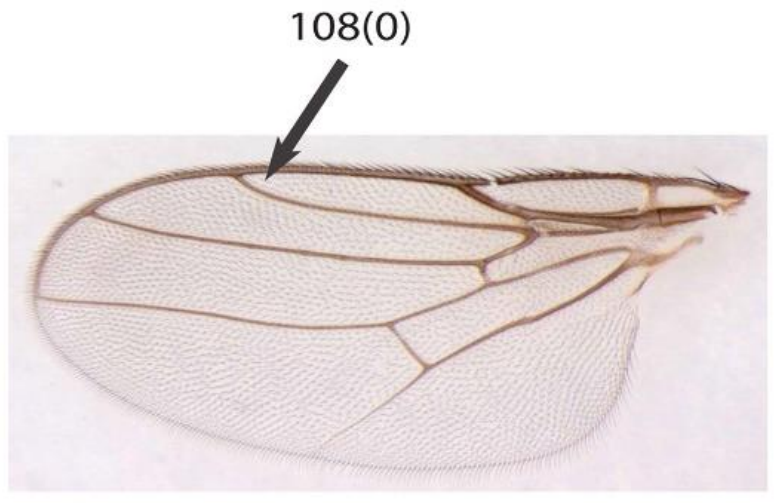

(a)

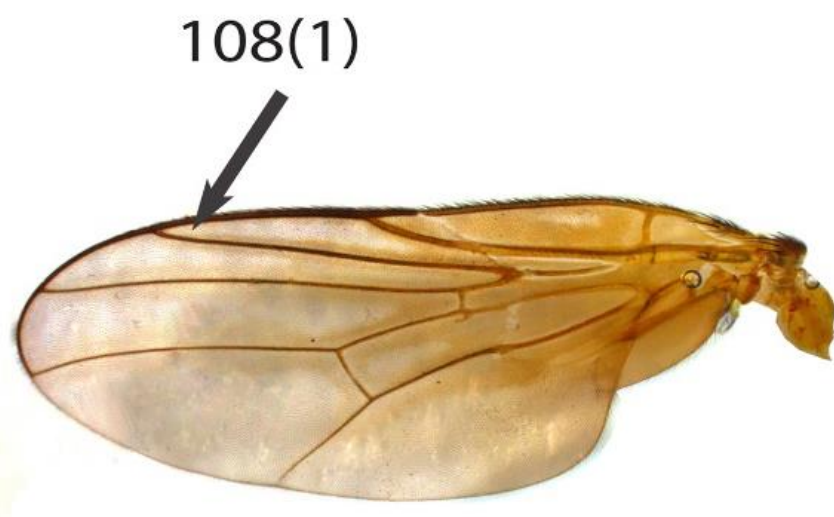

(b)

Figure 104a-b. Character 108, shape of anterior end of $\mathrm{R}_{2+3}$ : a. Metasiphonella sp., state 0 , curved to the $\mathrm{C} ; \mathrm{b}$. Batrachomyia nigritarsis, state 1 , about straight to the $\mathrm{C}$. 


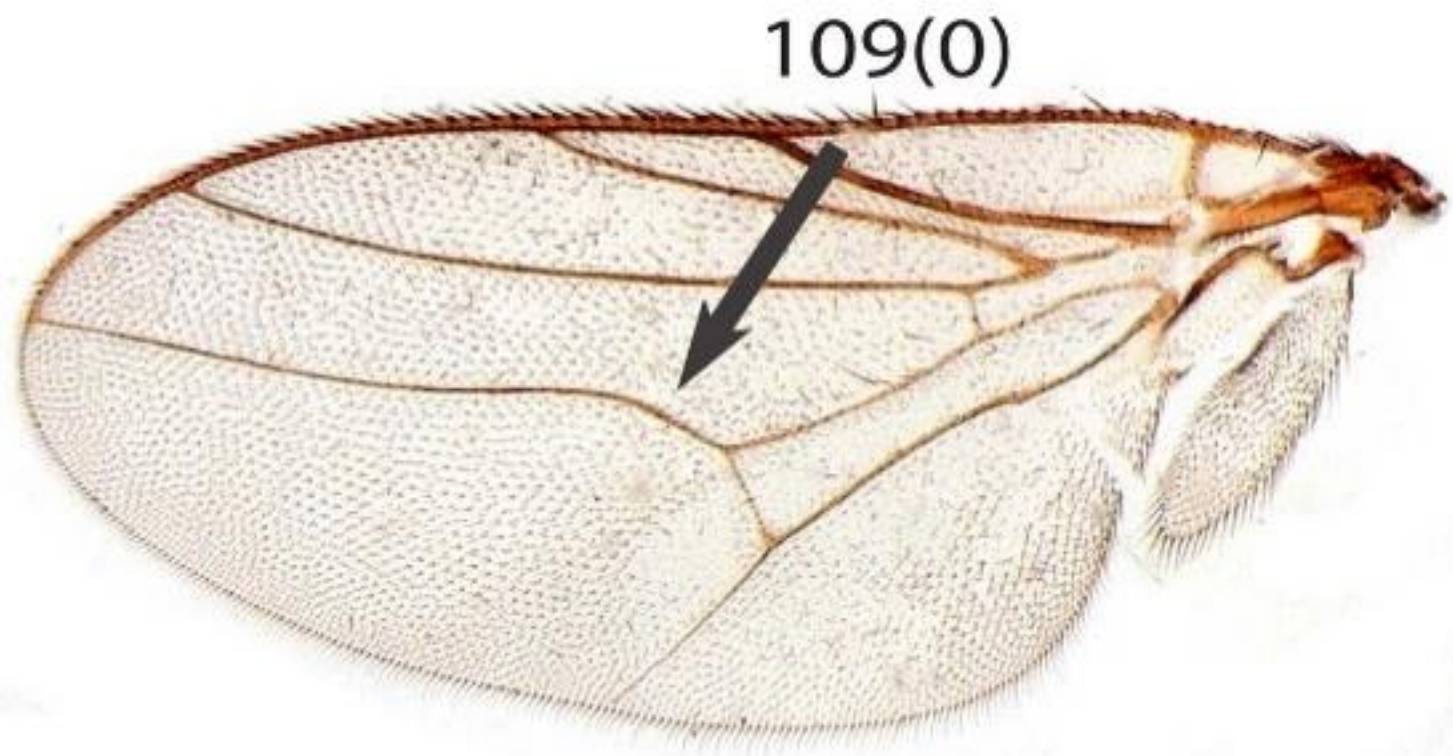

Figure 105: Character 109, shape of basal half of $\mathrm{M}_{1+2}$ : Rhodesiella sp.2, state 0 , convex anteriorly.

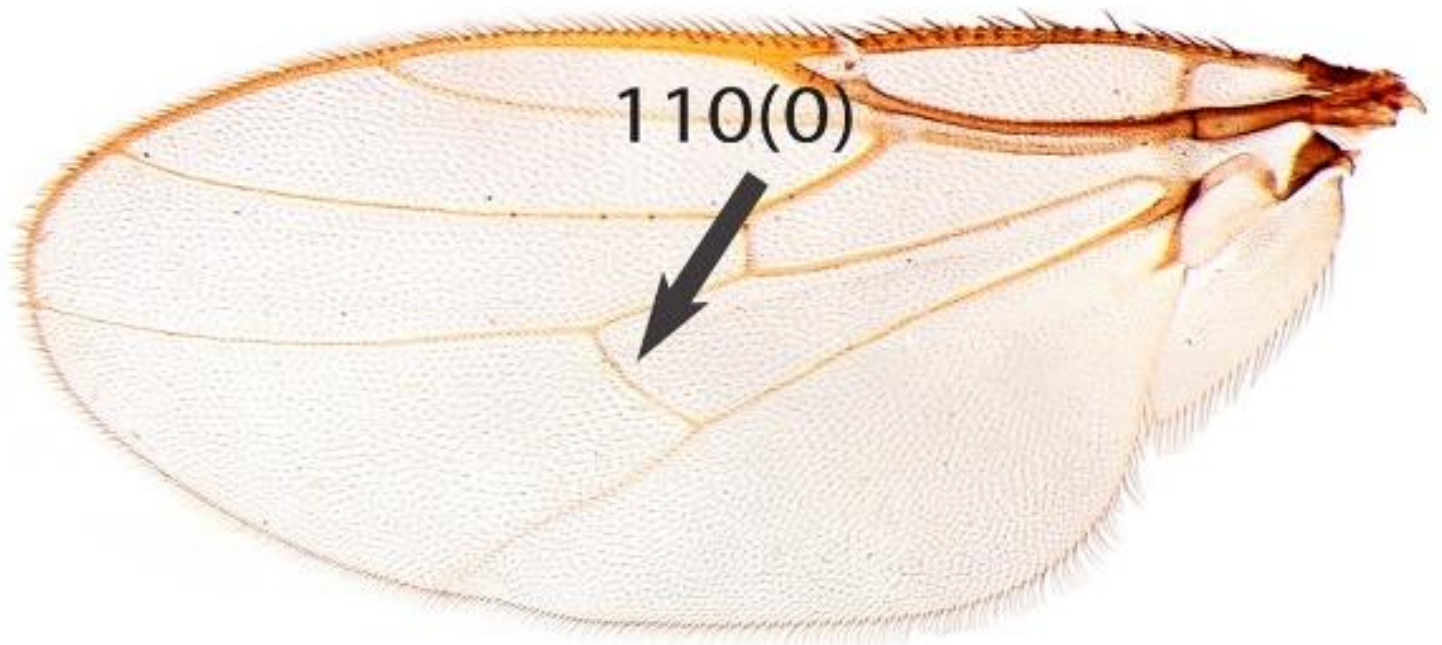

Figure 106: Character 110, shape of dm-cu: Polyodaspis ruficornis, state 0 , convex.

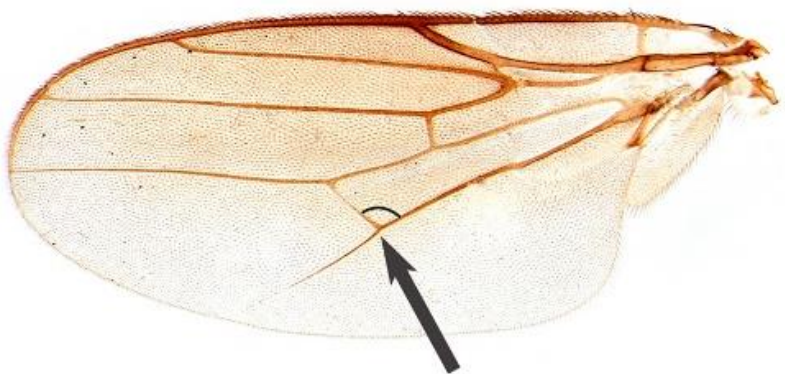

$111(0)$

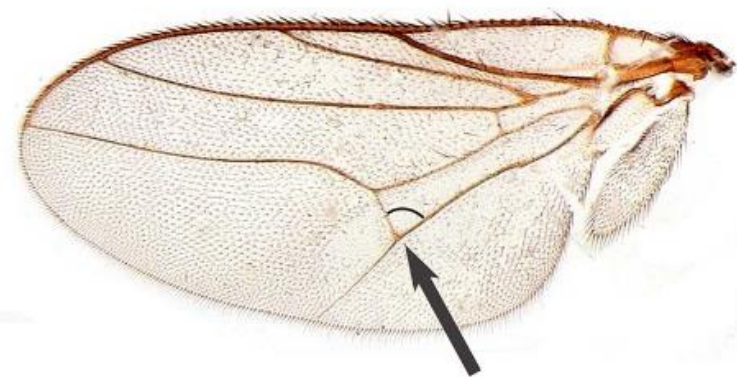

$111(1)$

(a)

(b)

Figure 107a-b. Character 111 , position of $\mathrm{dm}$-cu in relation to $\mathrm{CuA}_{1}$ : a. Lasiambia palposa, state 0 , more than $100^{\circ}$; b. Rhodesiella sp.2, state 1 , about $90^{\circ}$ or less. 


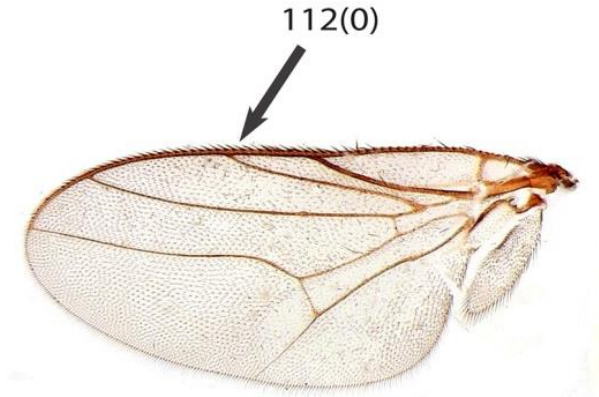

(a)

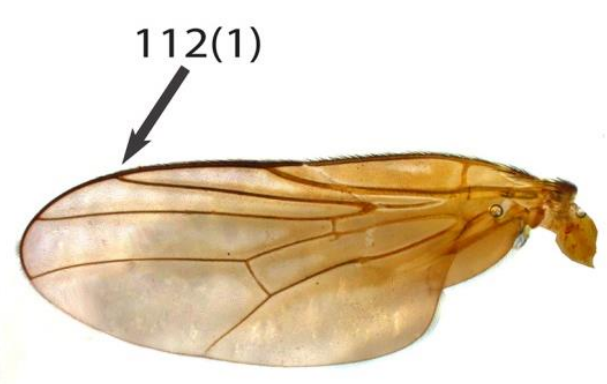

(b)

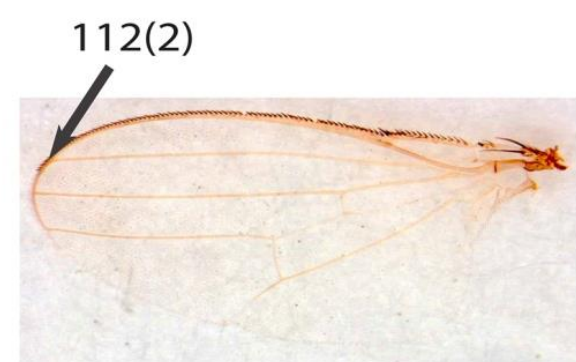

(c)

Figure 108a-c. Character 112, C2: a. Rhodesiella sp.2, state 0 , at most reaches to the middle of the wing; b. Batrachomyia nigritarsis, state 1, between middle and tip of the wing; c. Madiza glabra, state 2 , at the tip of the wing.

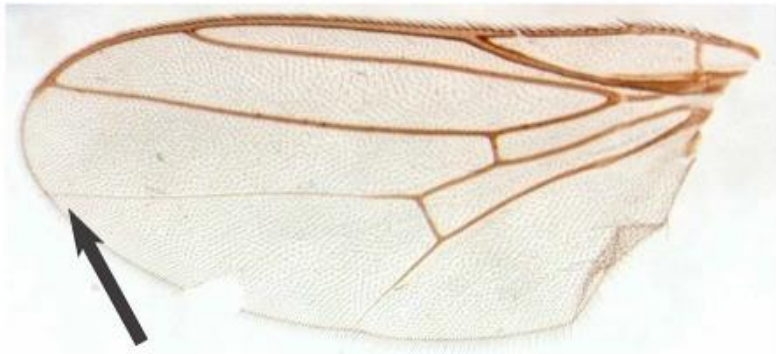

113(0)

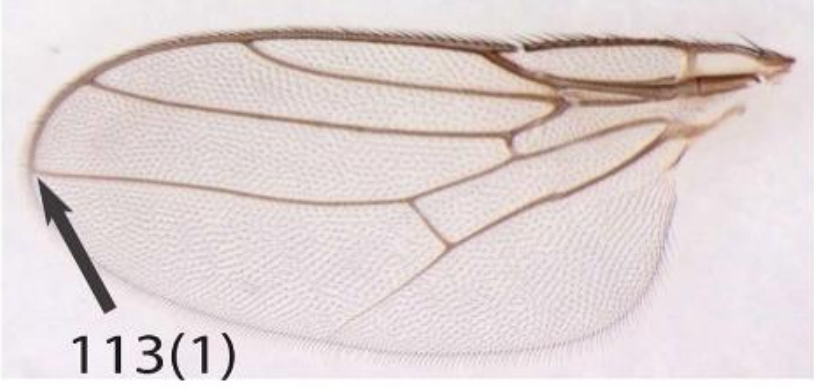

(a)

(b)

Figure 109a-b. Character 113, C4: a. Thaumatomyia gemina, state 0 , ending below of apical margin of the wing; b. Metasiphonella sp., state 1 , ending at the apical margin of the wing.

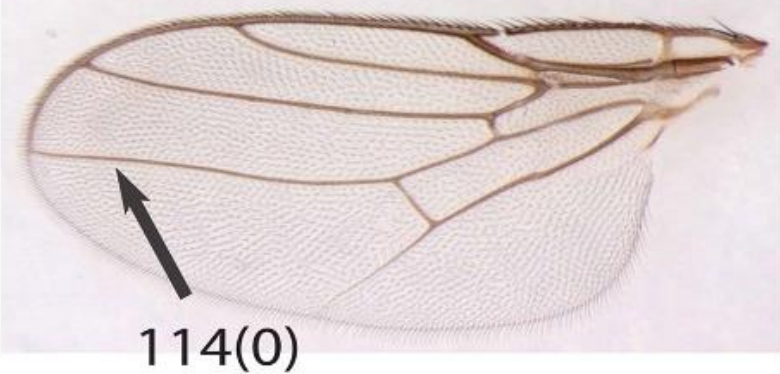

(a)

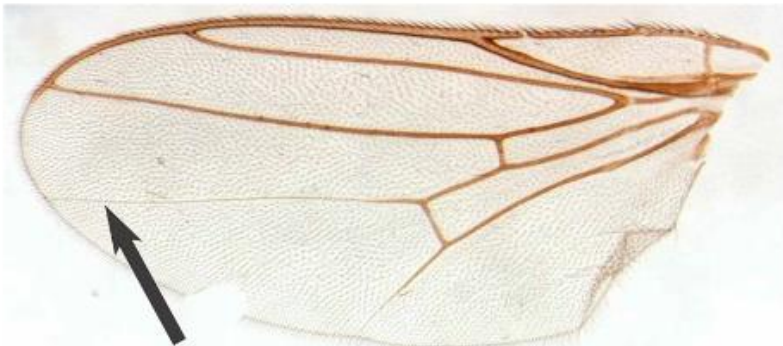

$114(1)$

(b)

Figure 110a-b. Character $114, \mathrm{M}_{1+2}$ thickness: a. Metasiphonella sp., state 0 , as thick as $\mathrm{R}_{4+5} ; \mathrm{b}$. Thaumatomyia gemina, state 1 , thinner than $\mathrm{R}_{4+5}$. 


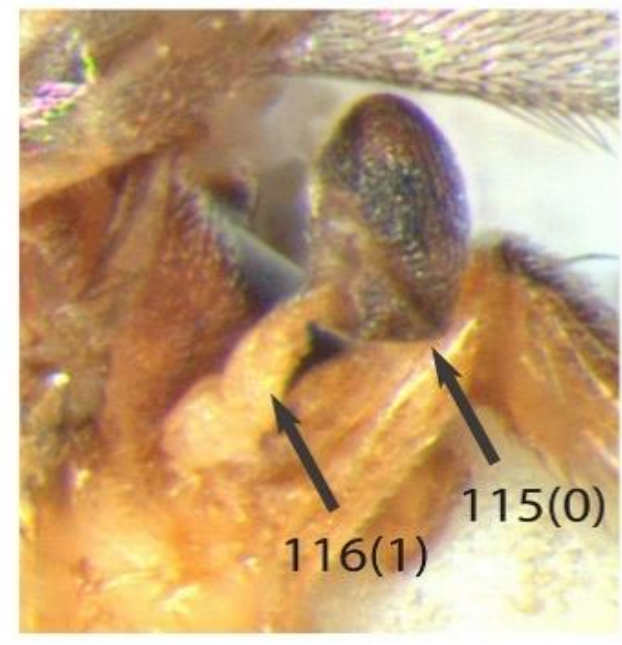

(a)

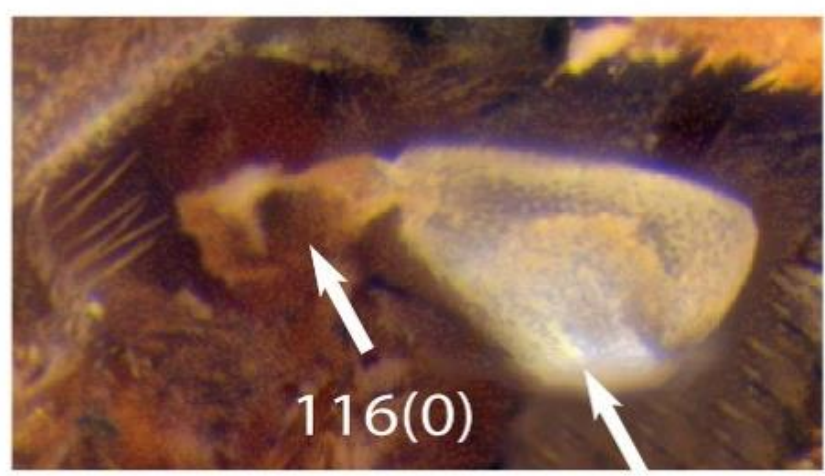

115(1)

(b)

Figure 111a-b. Character 115-116; C115, halter color (knob); C116, halter color (stem): a.

Pseudogaurax anchora, C115, state 0, black or dark brown; C116, state 1, yellow-ish or light brown; b. Heteroscinis hystrix, C115, state 1, yellow-ish or light brown; C116, state 0, black or dark brown.
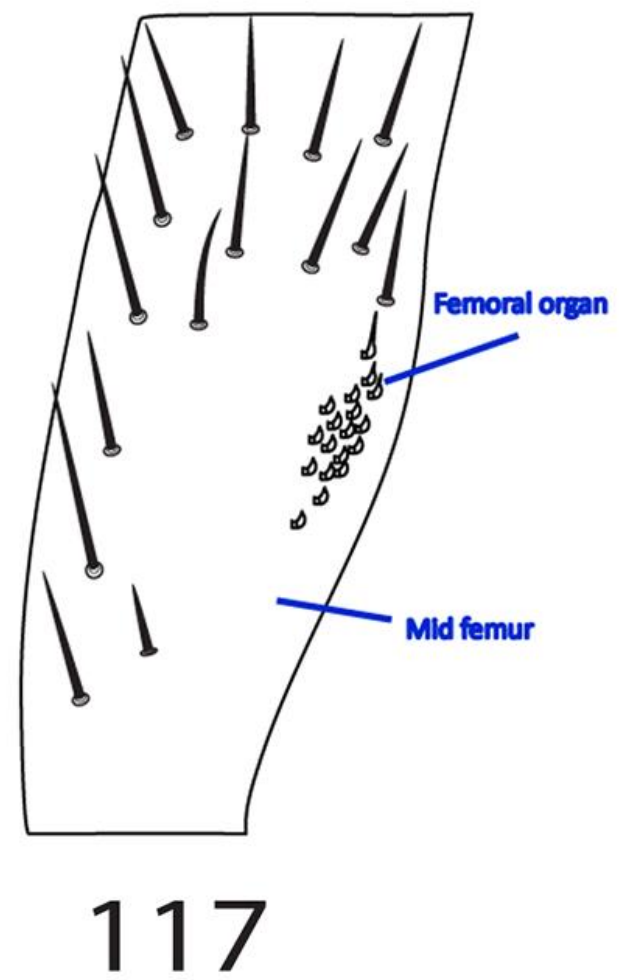

Figure 112: Character 117, male mid femoral organ: Schematic shape. 


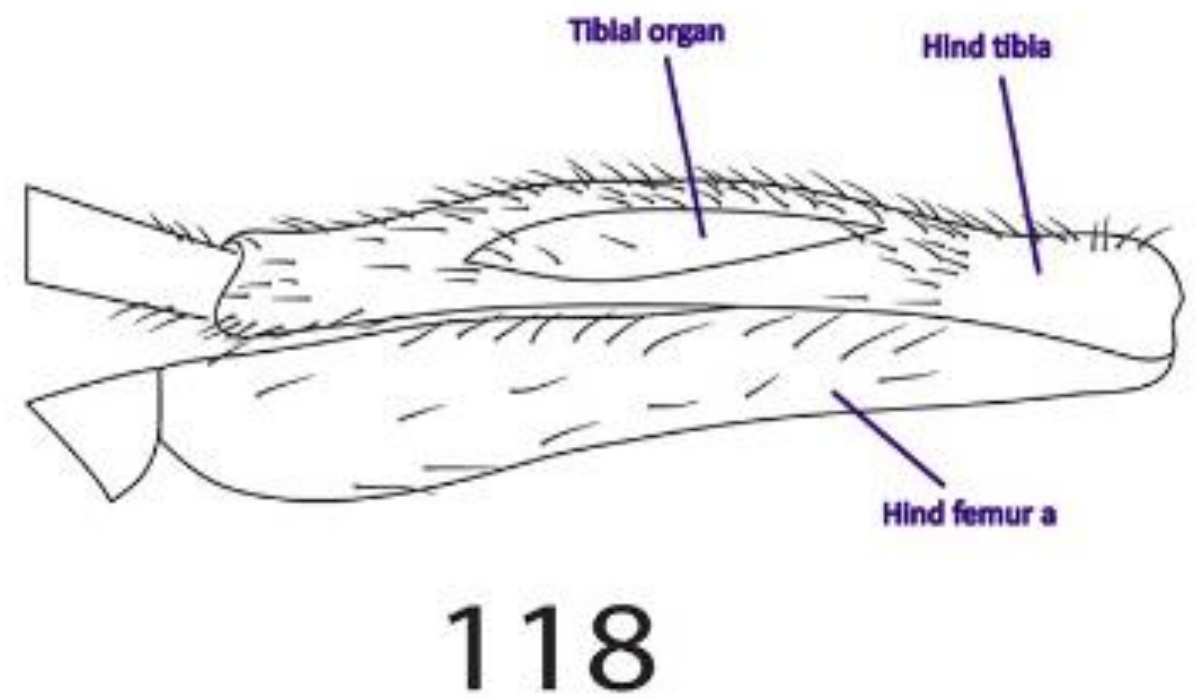

Figure 113: Character 118, tibial organ: Schematic shape.

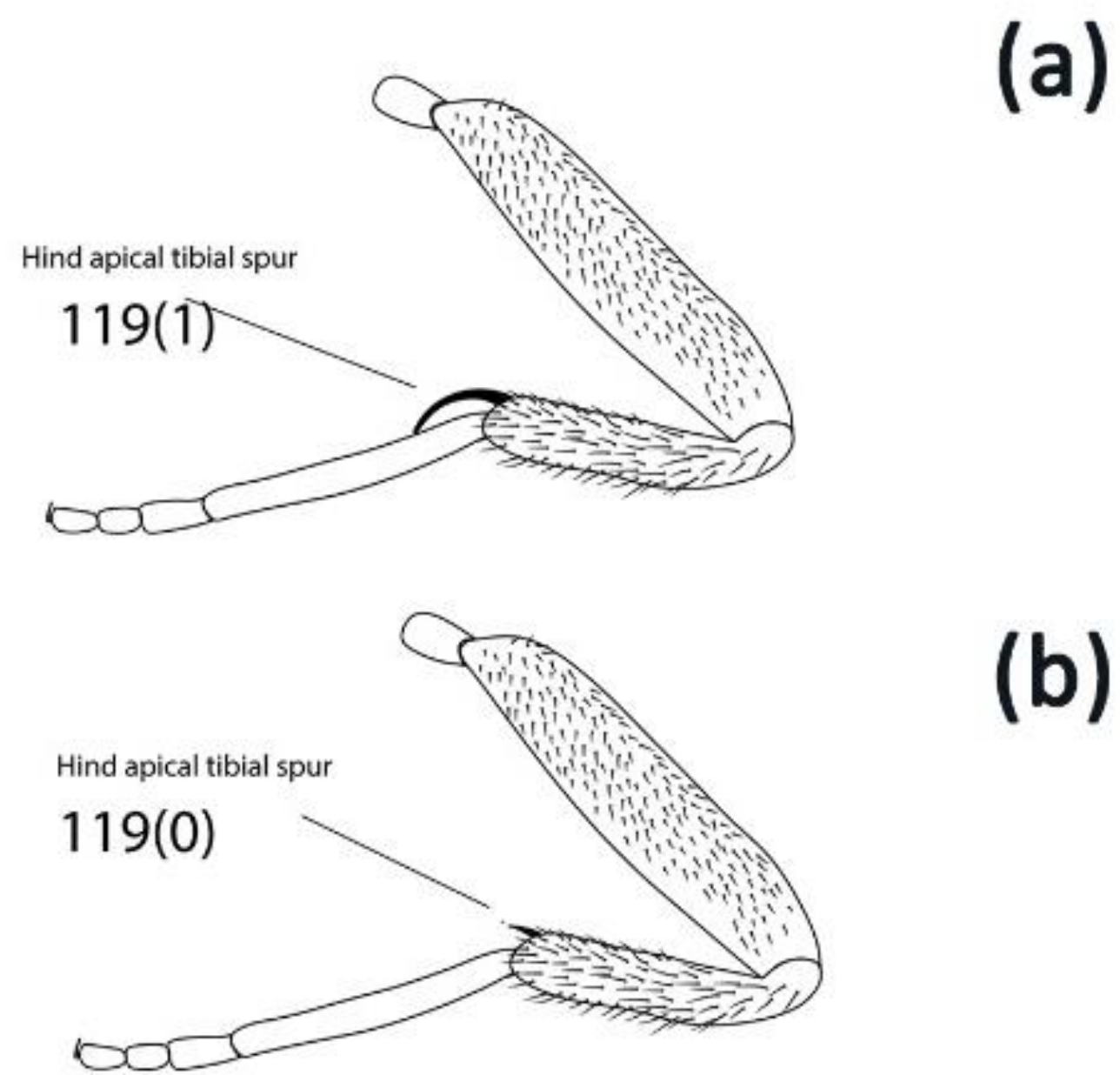

Figure 114a-b. Character 119, hind tibial spur: Schematic shape, a. state 1, clearly longer; b. state 0, shorter or slightly longer than hind tibia diameter. 


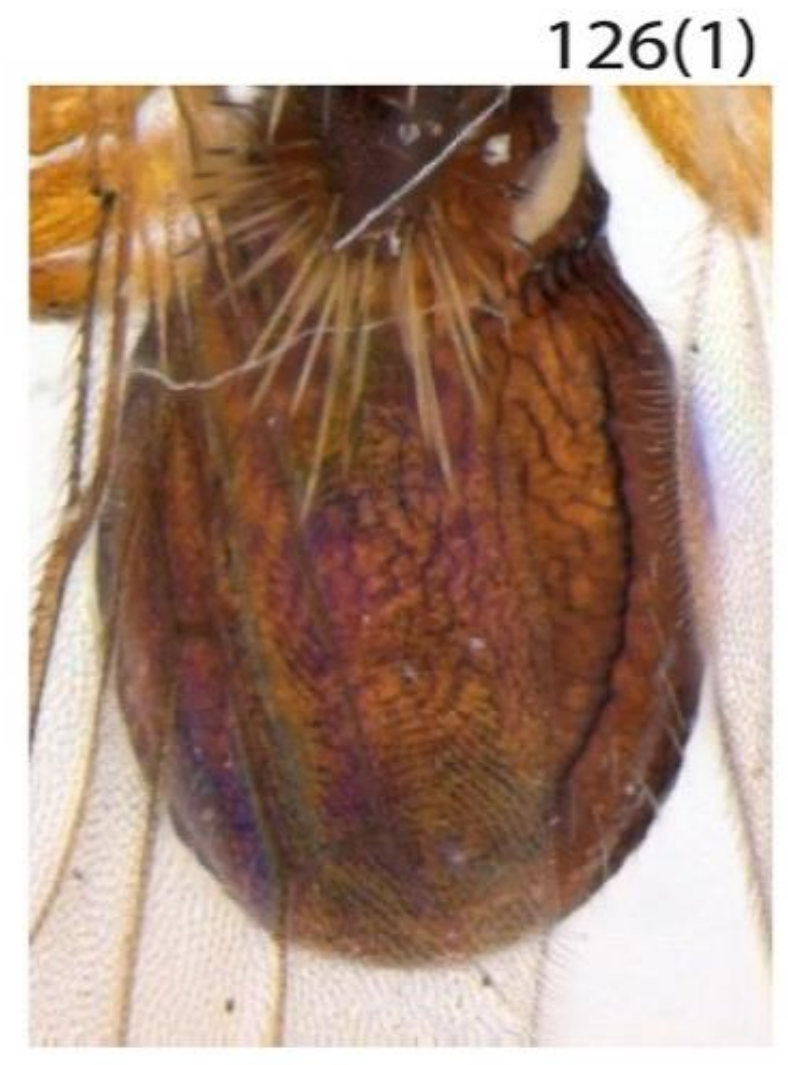

Figure 115: Character 126, abdominal tergites: Anatrichus erinaceus, state 1, syntergites 1+2 expanded comparing with other tergites.

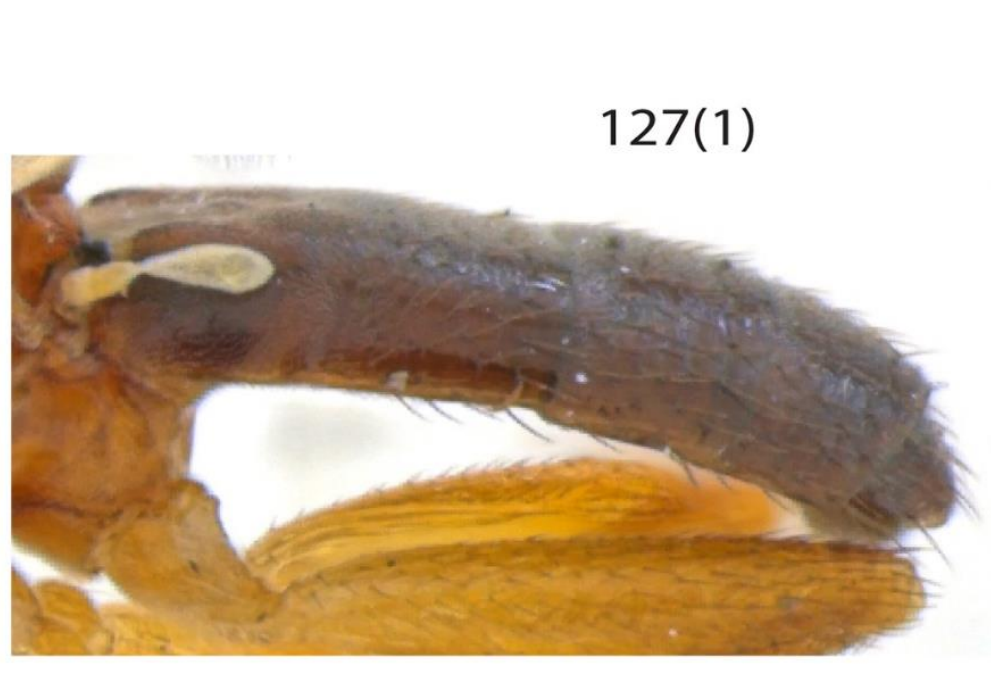

(a)

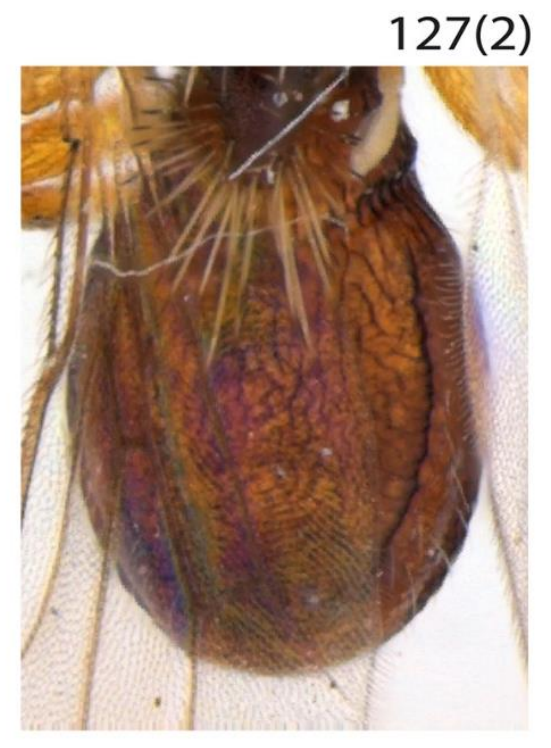

(b)

Figure 116a-b. Character 127, sclerotization of tergite: a. Elachiptera brevipennis, state 1, syntergites $1+2$ firmly sclerotized; b. Anatrichus erinaceus, state 2 , syntergites $1+2$ and tergite 3 firmly sclerotzed. 


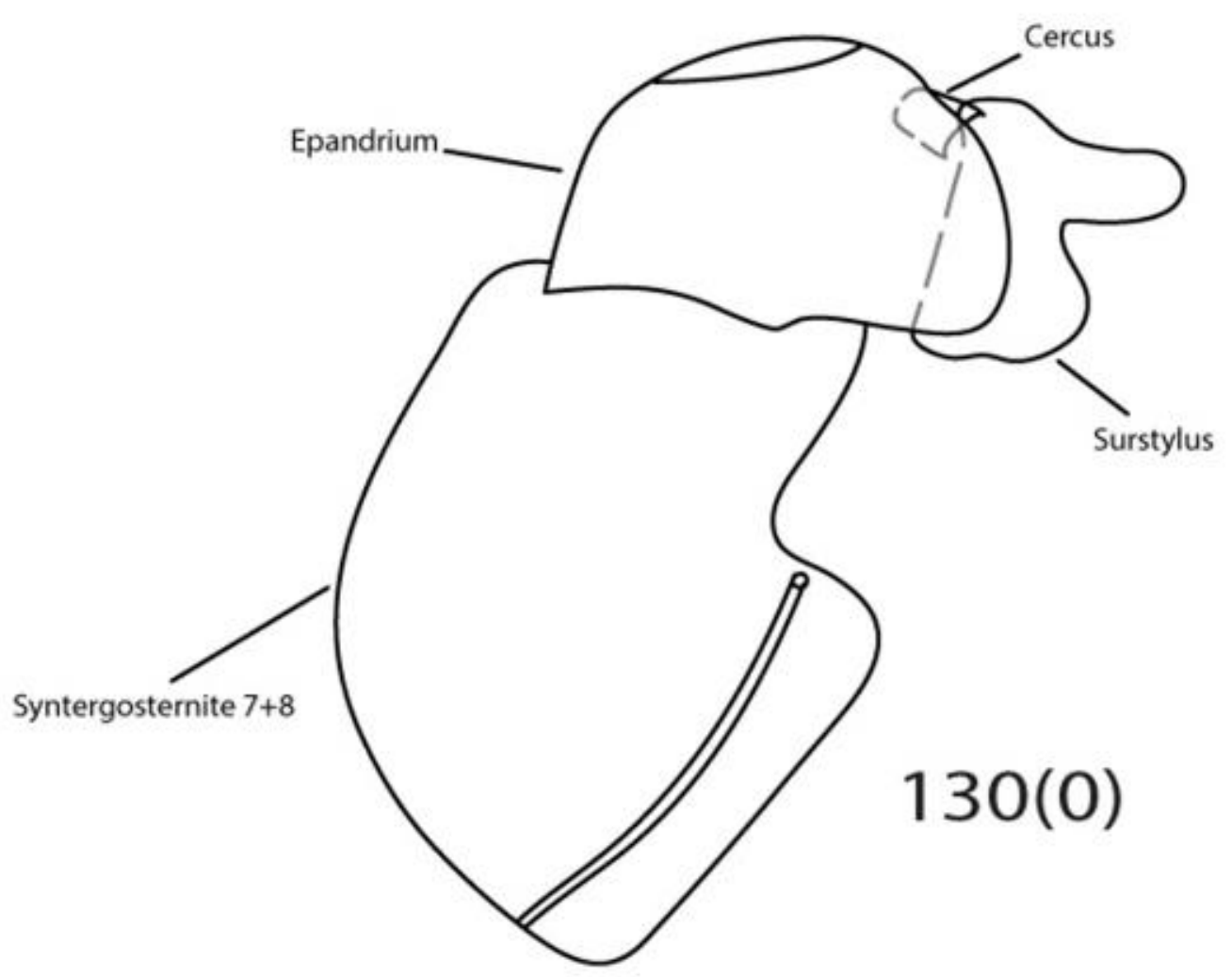

Figure 117: Character 130, syntergosternite 7+8: Dicraeus vagans, state 0 , broad.

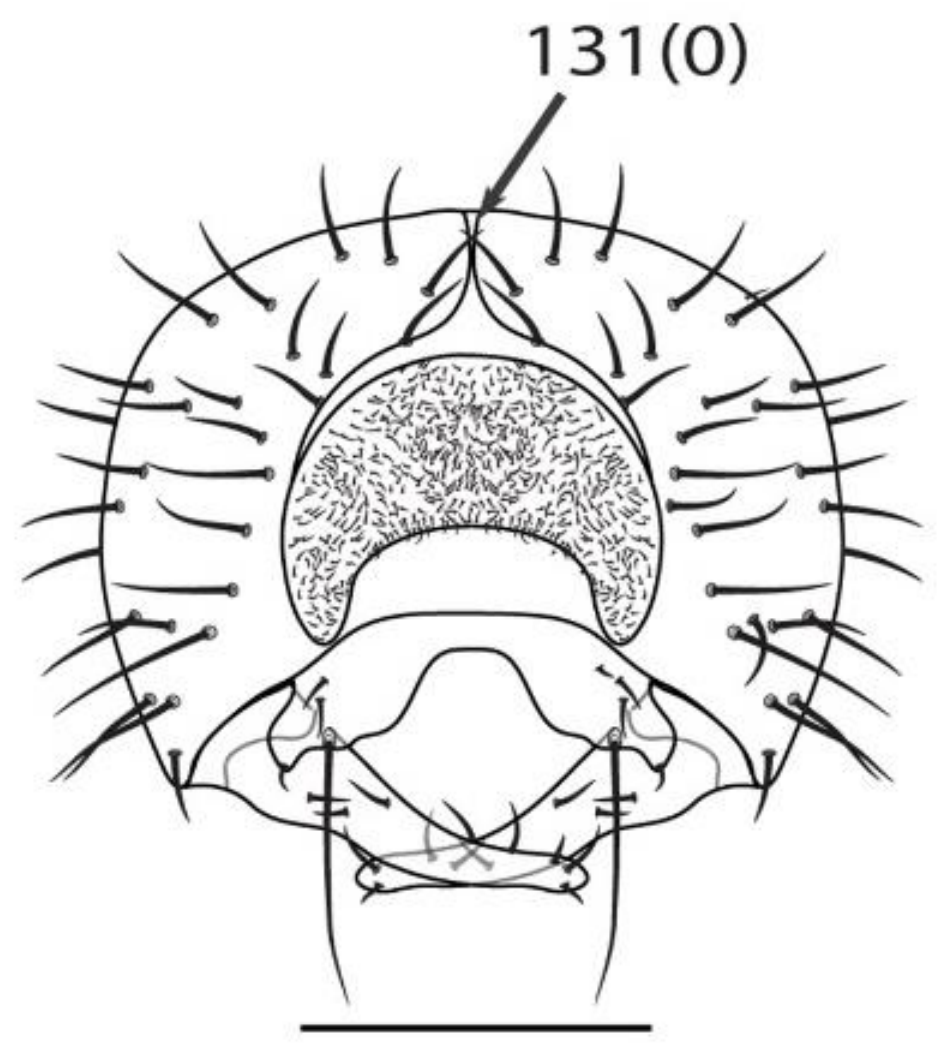

Figure 118: Character 131, epandrium medial line (dorsal sulcus): Elachiptera brevipennis, state 0 , present. 


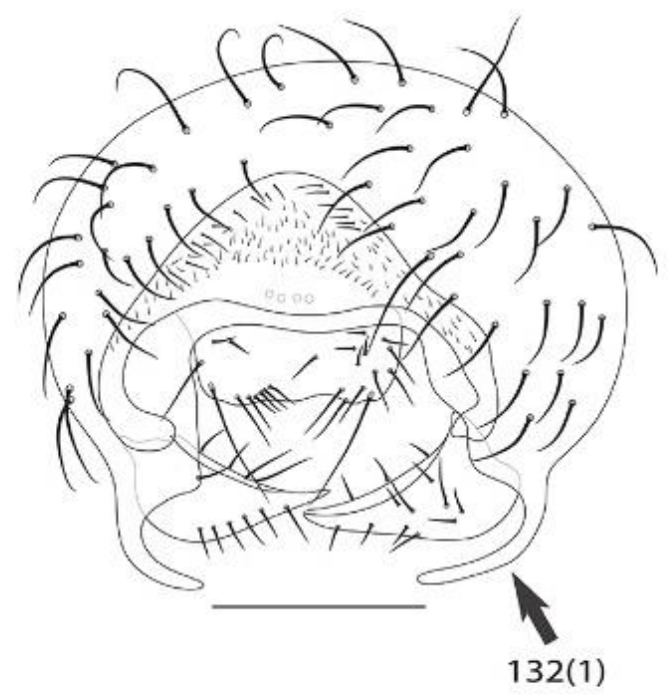

(a)

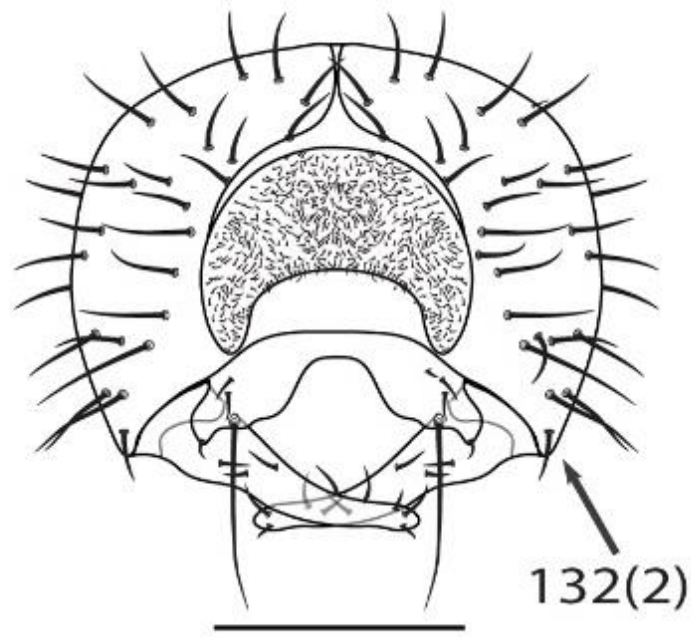

(b)

Figure 119a-b. Character 132, antero-ventral corner of epandrium in posterior view: a. Apotropina sp. nov., state 1, with a long projection; b. Elachiptera brevipennis, state 2, absent.

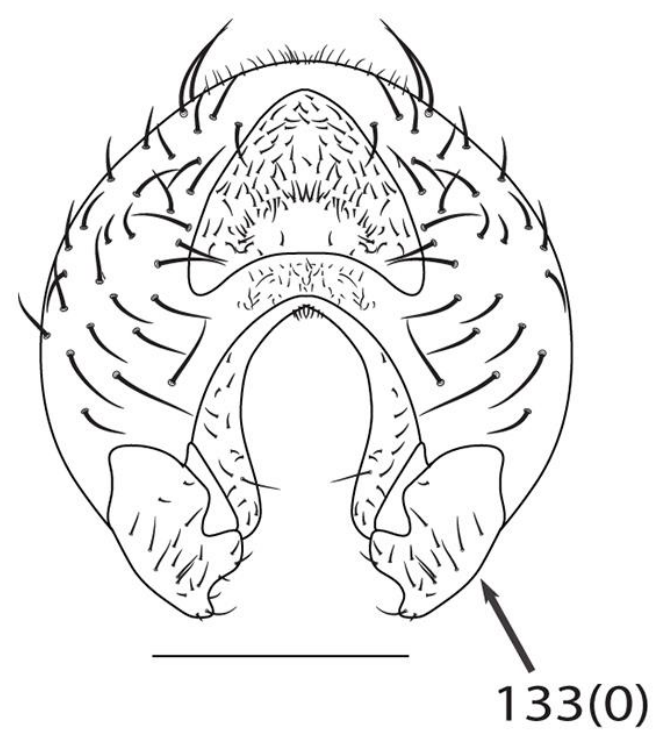

(a)

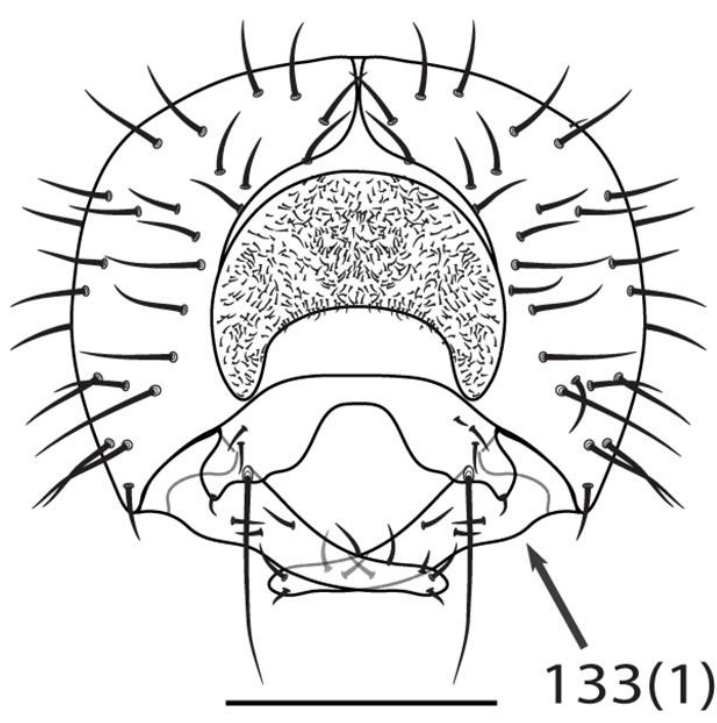

(b)

Figure 120a-b. Character 133, size of surstylus (comparing with the size of epandrium): a. Apallates dissidens, state 0 , short; b. Elachiptera brevipennis, state 1 , long. 


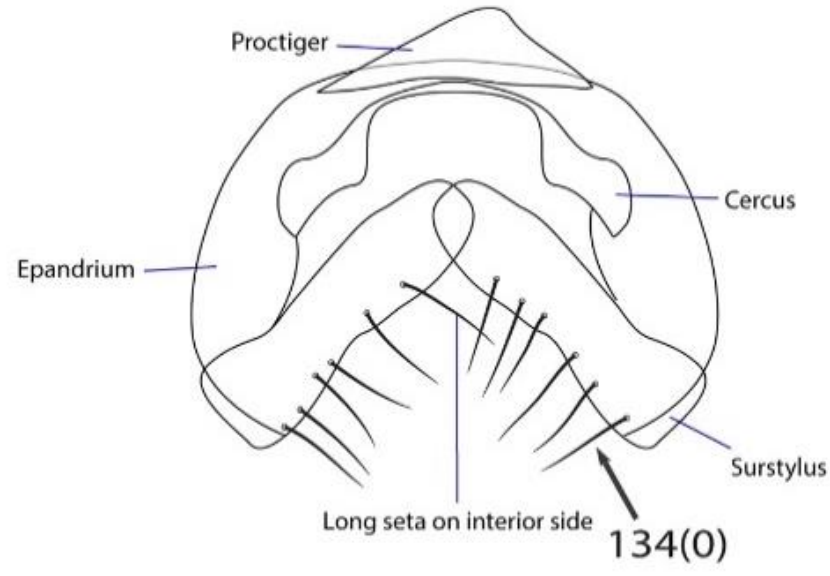

(a)

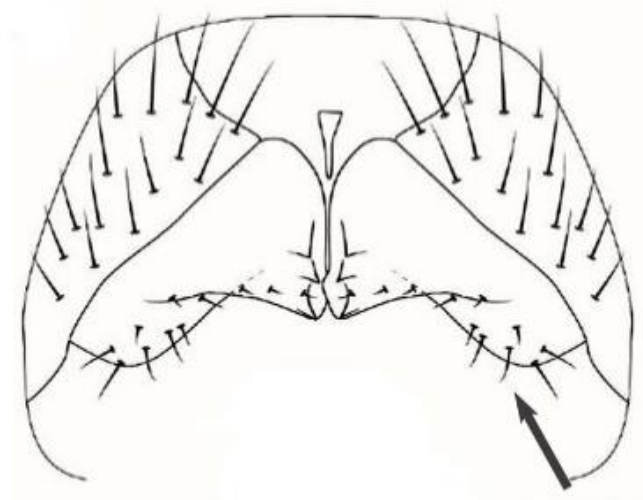

134(1)

(b)

Figure 121a-b. Character 134, elongated setae at the edge of anterior lateral margins of surstylus: a. Disciphus peregrinus, state 0, present; b. Thaumatomyia gemina (Riccardi 2016, modified), state 1 , absent.

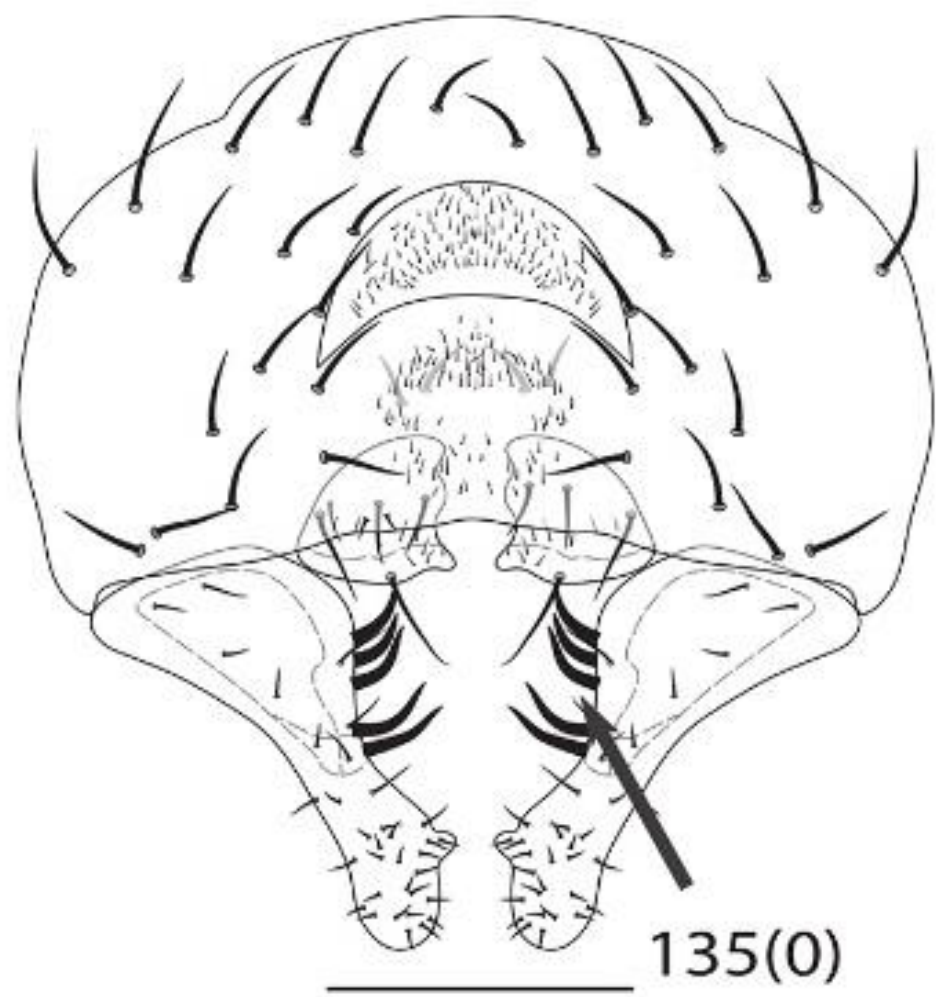

Figure 122: Character 135, elongated setae at lateral margins of surstylus modified to spines: Dicraeus vagans, state 0 , present. 


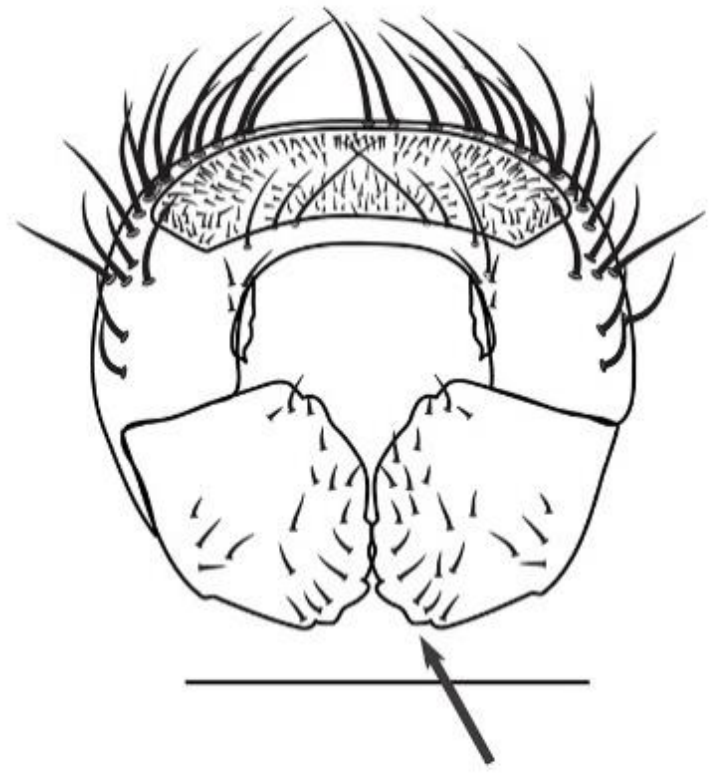

$136(1)$

(a)

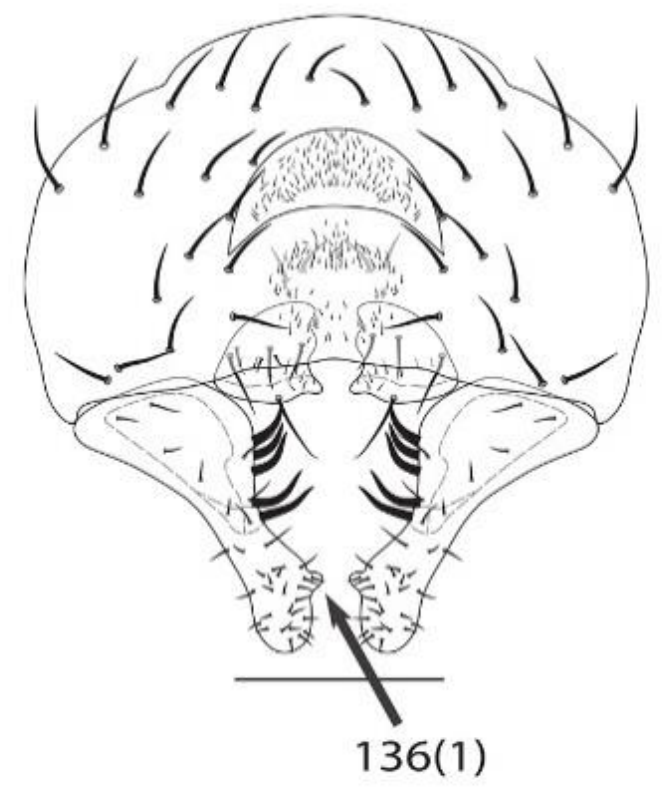

(b)

Figure 123a-b. Character 136, marginals of surstylus in posterior view: a. Biorbitella hesperia, state 1 , indentured; b. Dicraeus vagans, state 1. 


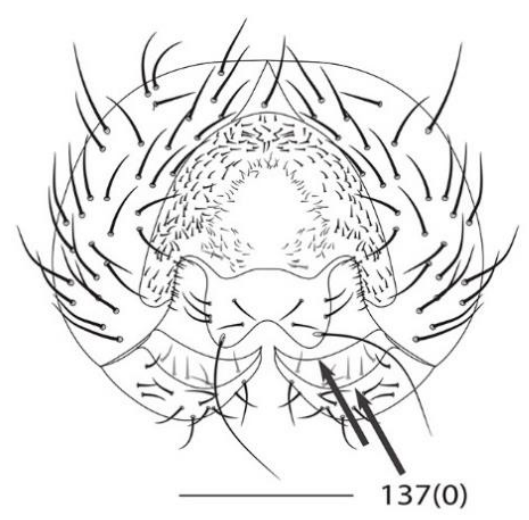

(a)

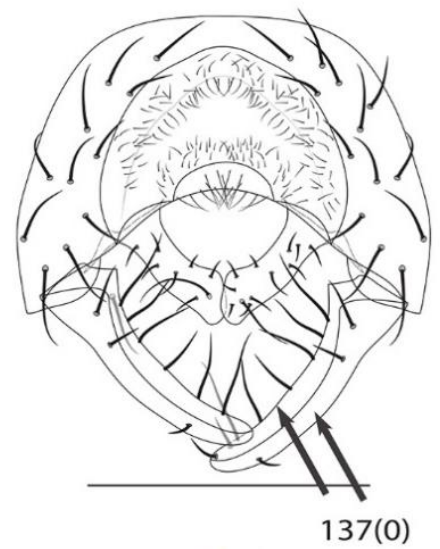

(b)

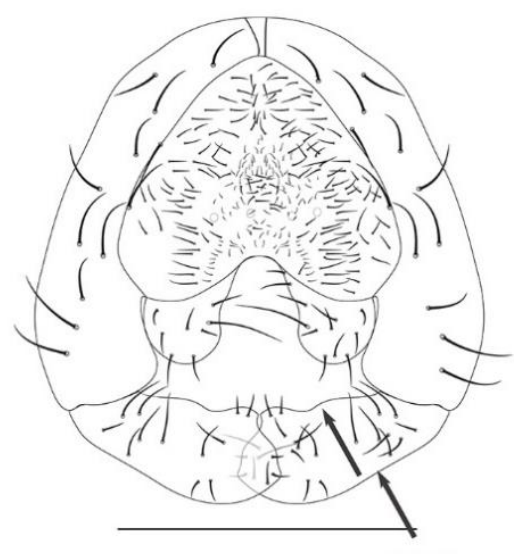

(d)

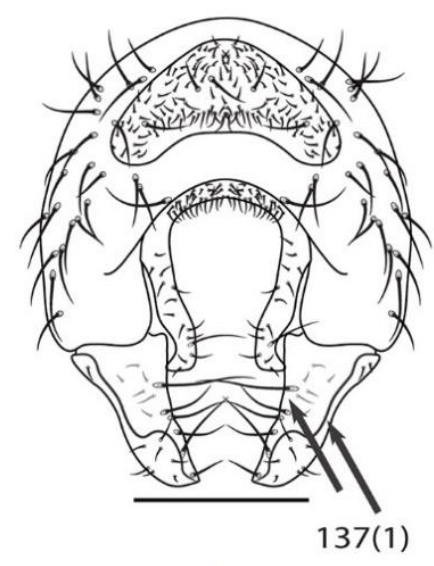

(c)

Figure 124a-d. Character 137, anterior and posterior lateral margins of surstylus: a. Calamoncosis minima, state 0 , parallel; b. Conioscinella solute, state 0 ; c. Hippelates plebejus, state 1 , convergent; d. Microcercis albipalpis, state 2, divergent. 


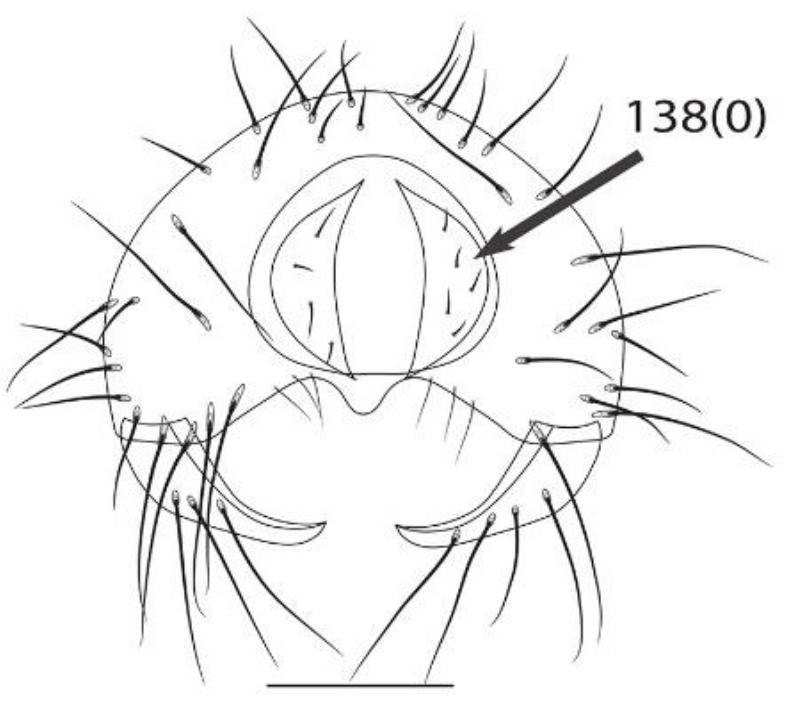

(a)

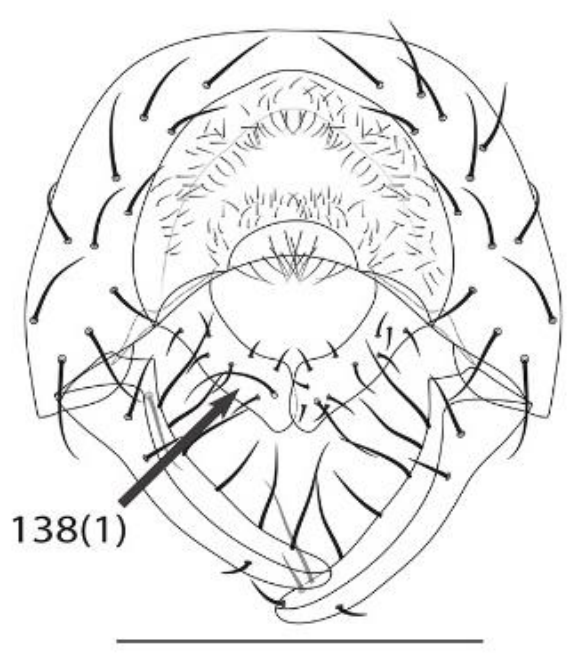

(b)

Figure 125a-b. Character 138, location of cercus on epandirum: a. Meoneura flavifacies (Drawn by Paula Raile Riccardi), state 0, placed on proctiger, free; b. Conioscinella solute, state 1, bellow proctiger, connected with epandrium.

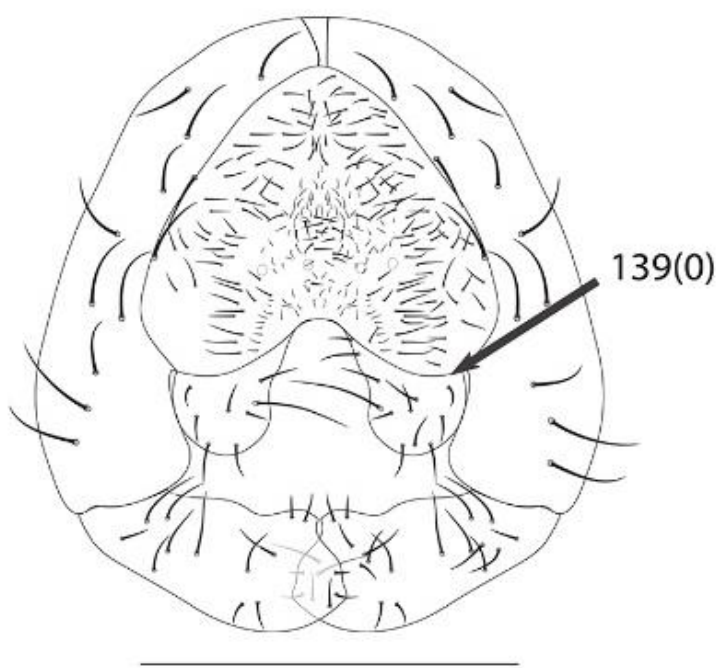

(a)

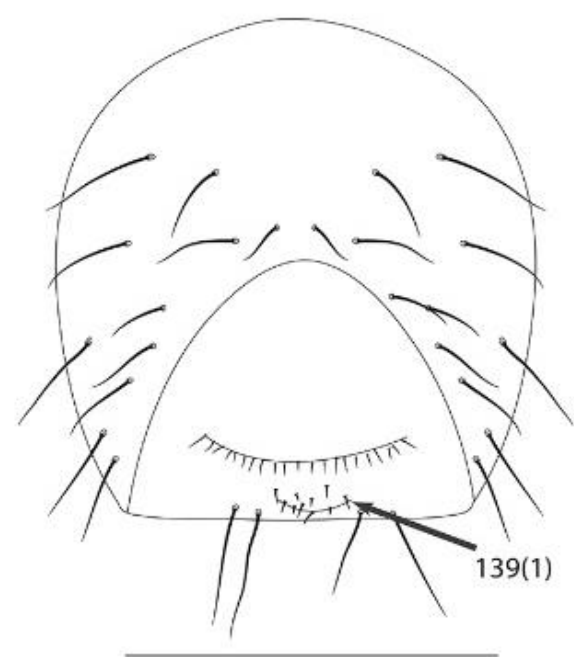

(b)

Figure 126a-b. Character 139, cercus articulation: a. Microcercis albipalpis, state 0, articulated; b. Chloropella bipartita (Drawn by Paula Raile Riccardi), state 1, fused to epandirum. 


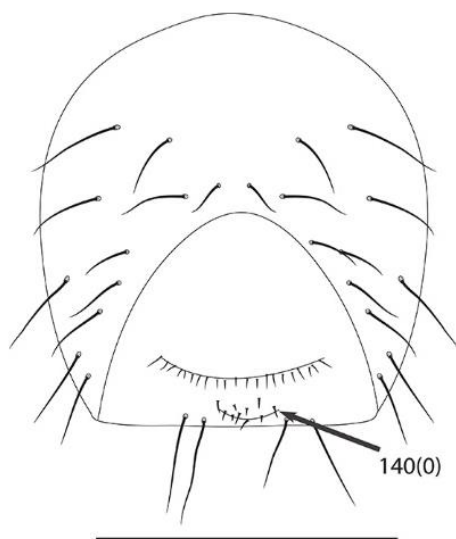

(a)

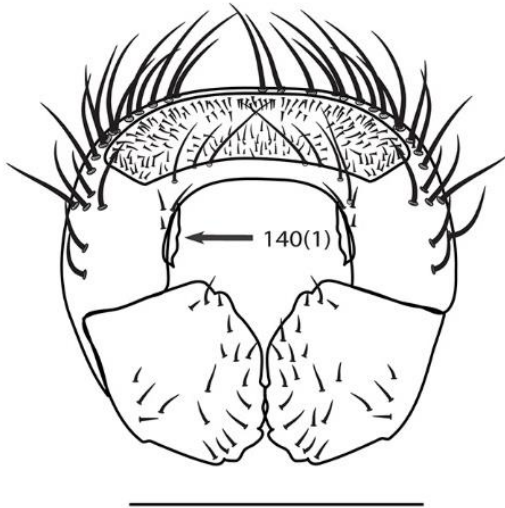

(b)

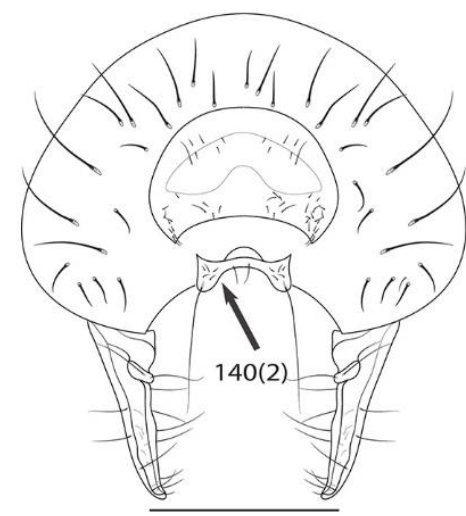

(c)

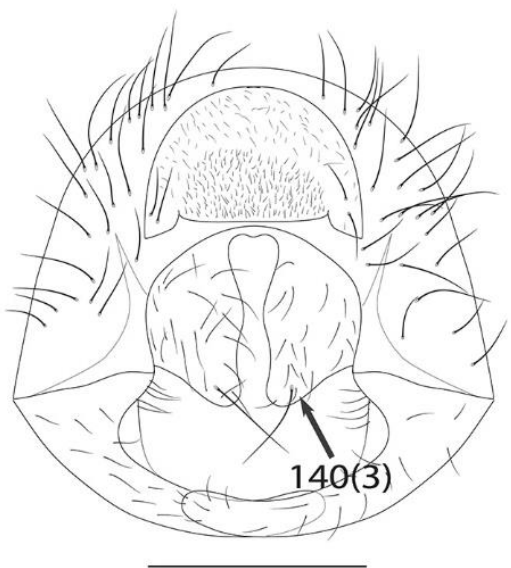

(d)

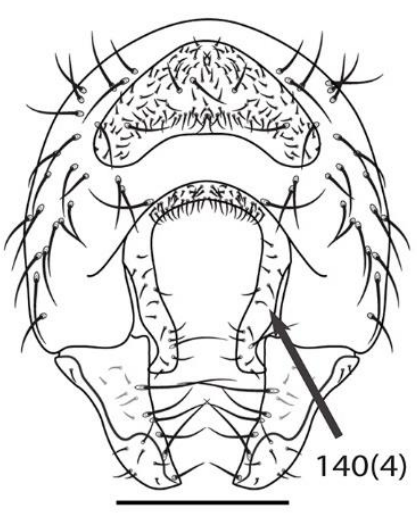

(e)

Figure 127a-e. Character 140, length of cercus: a. Chloropella bipartita (Drawn by Paula Raile Riccardi), state 0 , almost vestigial, in the middle; b. Biorbitella hesperia, state 1 , almost vestigial, in laterals; c. Sepsidoscinis maculipennis, state 2, small; d. Oscinella frit, state 3, enlarged, broad; e. Hippelates plebejus, state 4, enlarged, narrow.

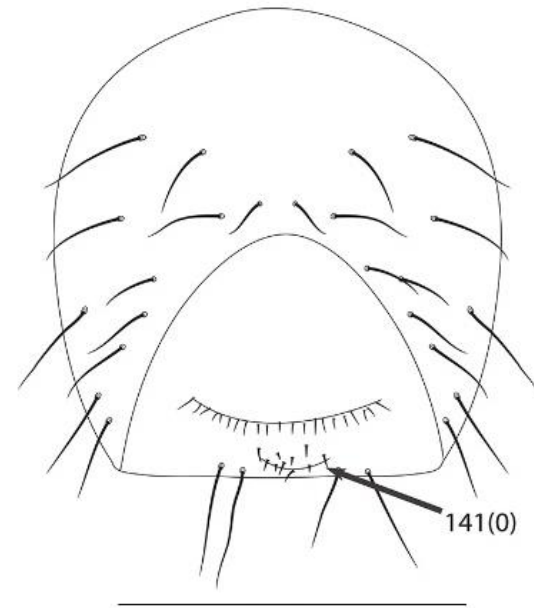

(a)

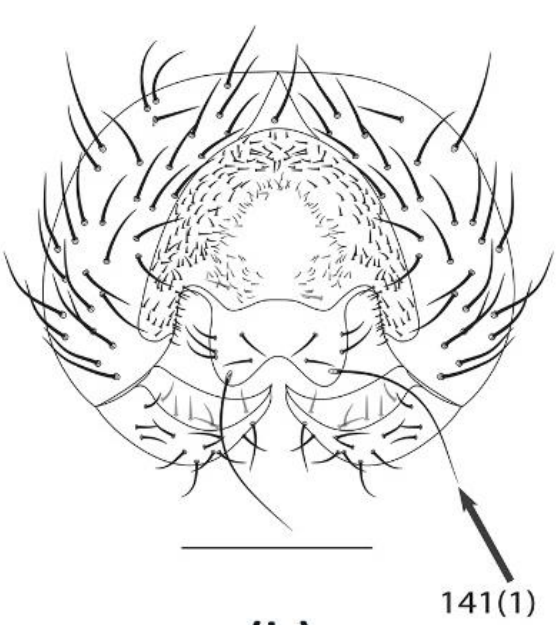

(b)

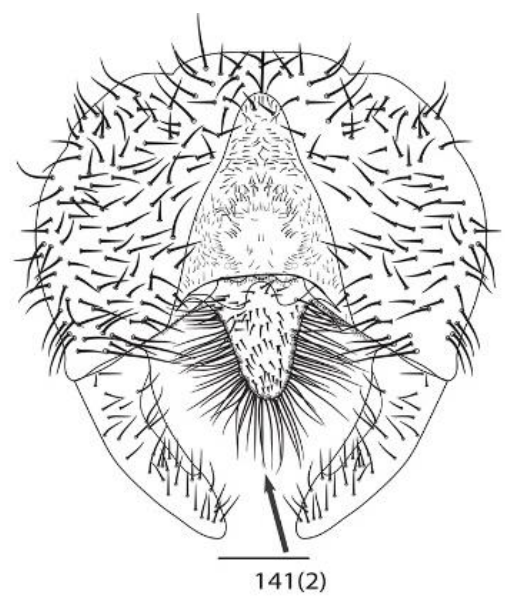

(c)

Figure 128a-c. Character 141, elongated setae at tip of cercus: a. Chloropella bipartita (Drawn by Paula Raile Riccardi), state 0, absent; b. Calamoncosis minima, state 1, present, 1 to 3; c. Lipara pullitarsis, state 2 , present, several. 


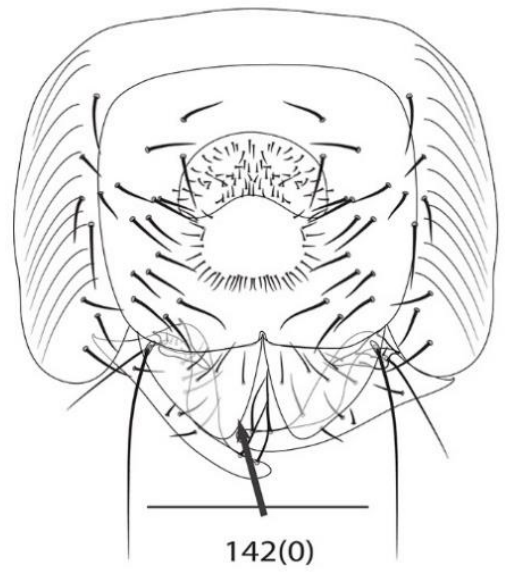

(a)

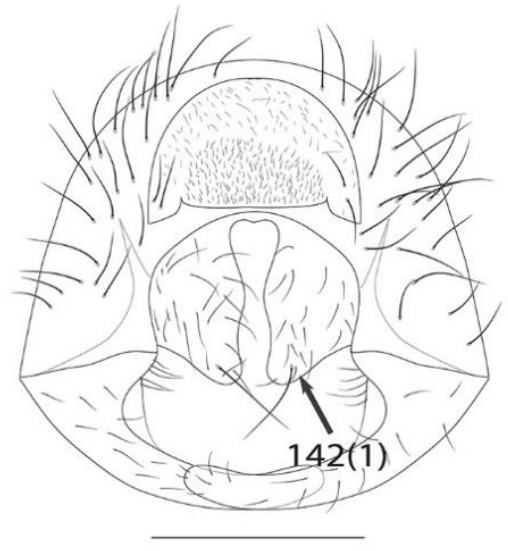

(b)

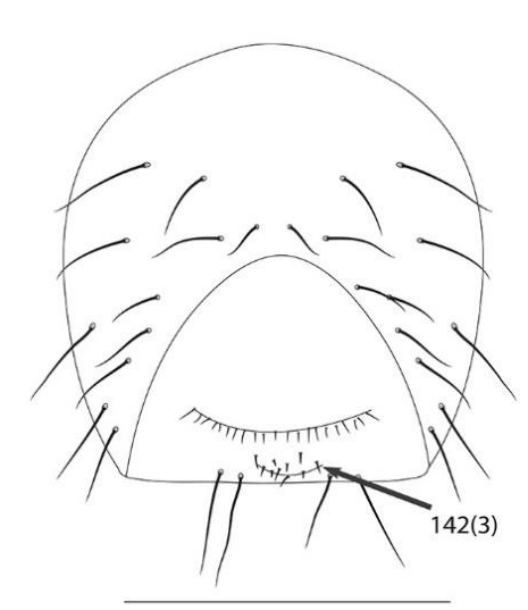

(d)

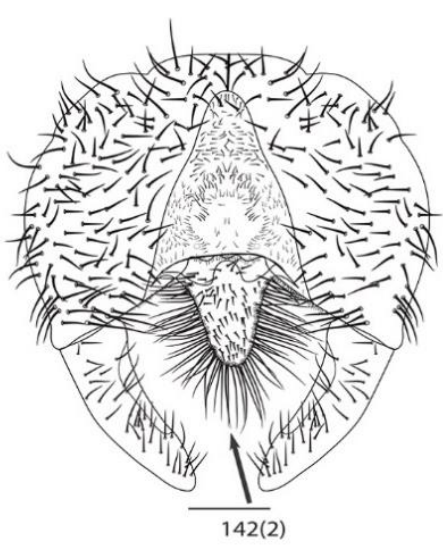

(c)

)

- 


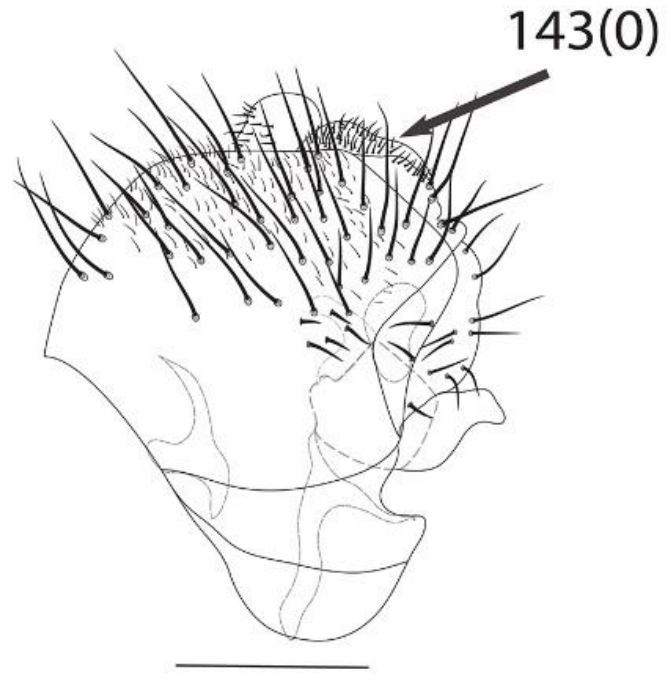

(a)

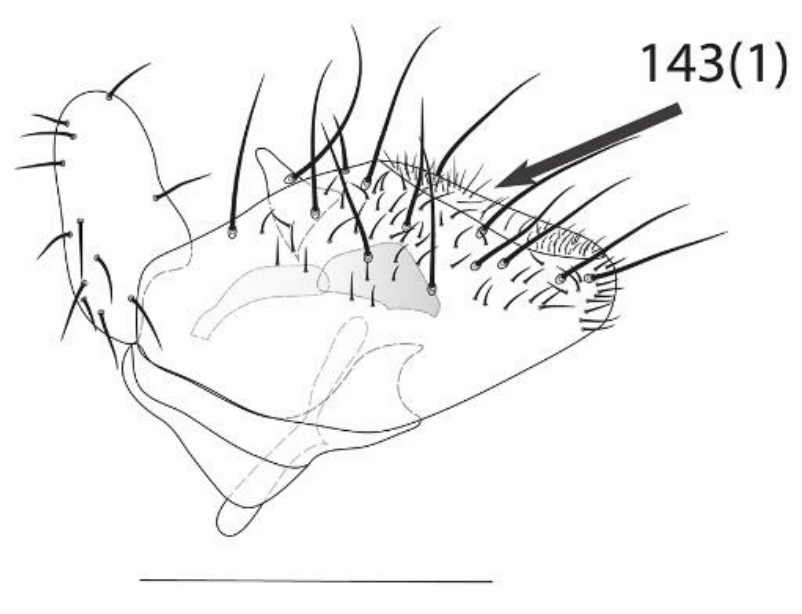

(b)

Figure 130a-b. Character 143, proctiger: a. Calamoncosis minima, state 0 , projected dorsally; b. Conioscinella soluta, state 1 , almost flat, not visible laterally.

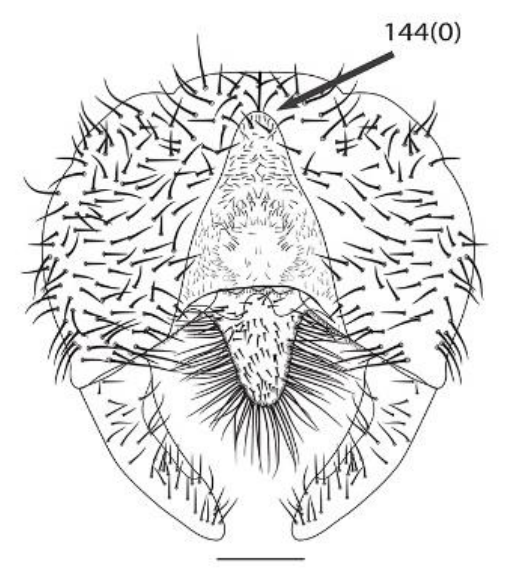

(a)

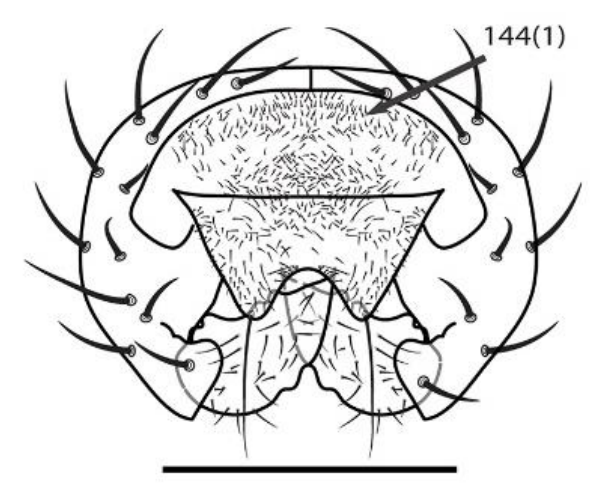

(b)

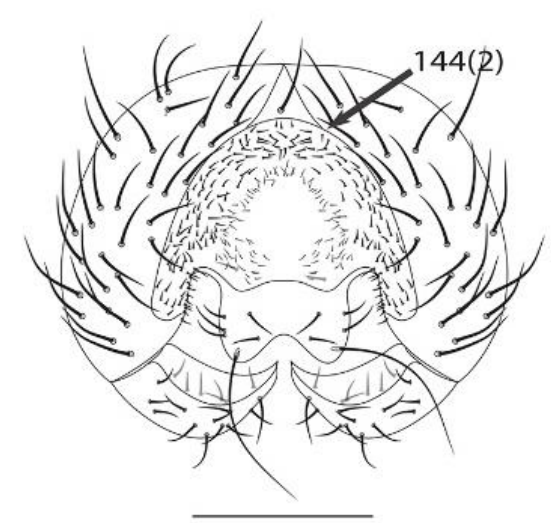

(c)

Figure 131a-c. Character 144, proctiger in posterior view: a. Lipara pullitarsis, state 0, elongated dorsoventrally; b. Loxobathmis obliquans, state 1, widened laterally; c. Calamoncosis minima, state 2 , almost as long as wide. 


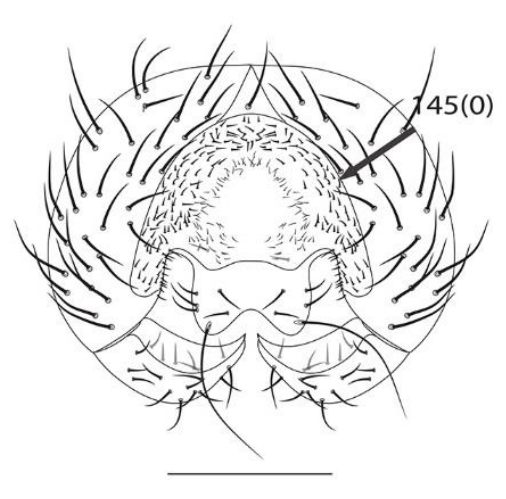

(a)

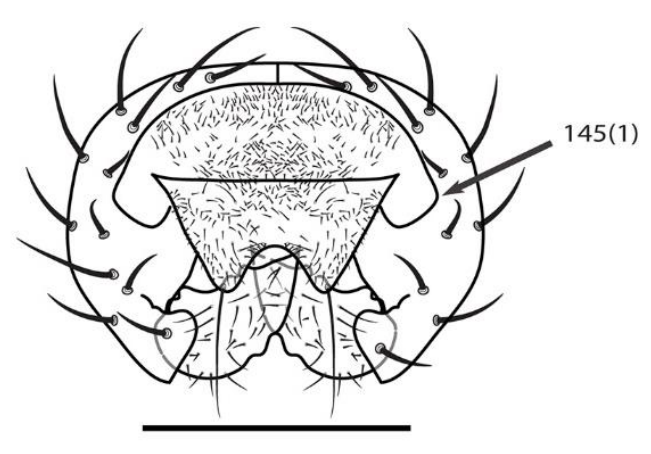

(b)

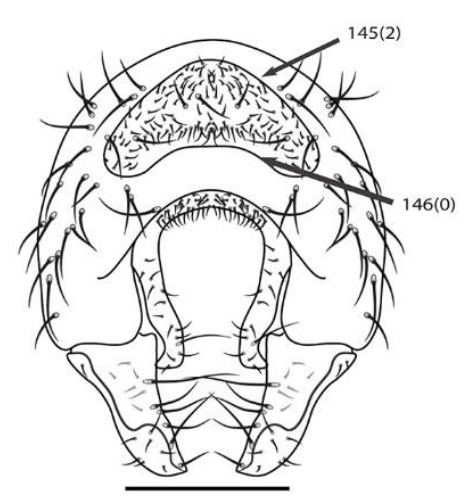

(c)

Figure 132a-c. Characters 145-146; C145, shape of proctiger in lateral margins (posterior view); C146, ventral margin of proctiger: a. Calamoncosis minima, C145, state 0, concave; b. Loxobathmis obliquans, C145, state 1 , almost $90^{\circ}$; c. Hippelates plebejus, C145, state 2, almost straight; C146, state 0 , concave.

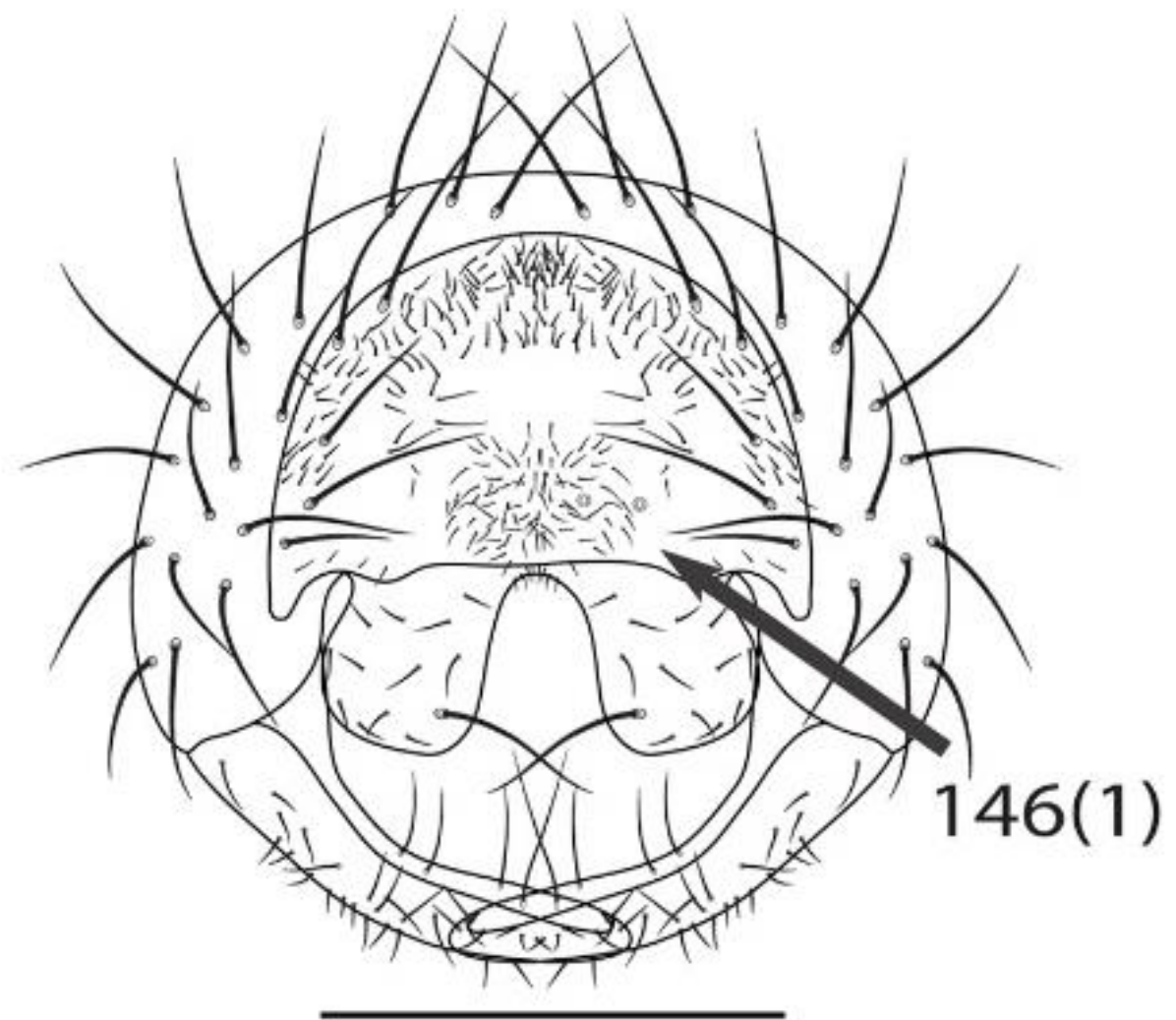

Figure 133: Character 146, ventral margin of proctiger: Pseudogaurax anchora, state 1, straight. 


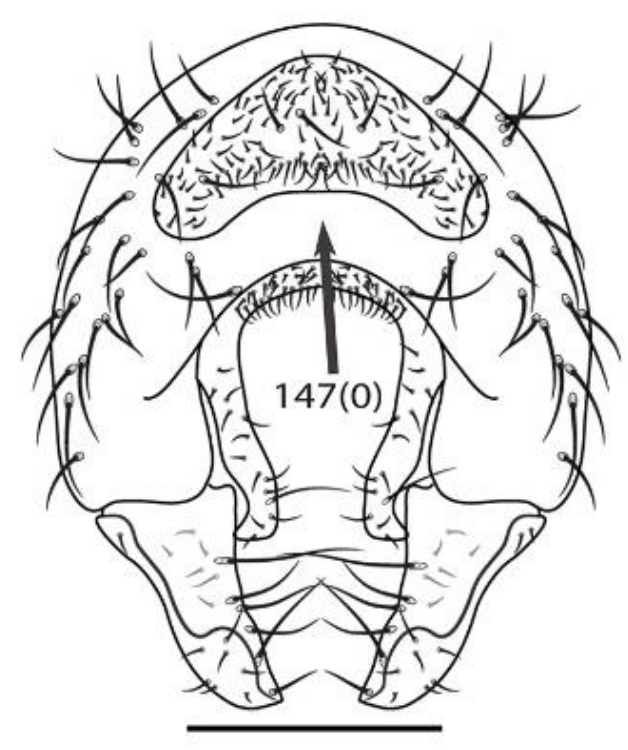

(a)

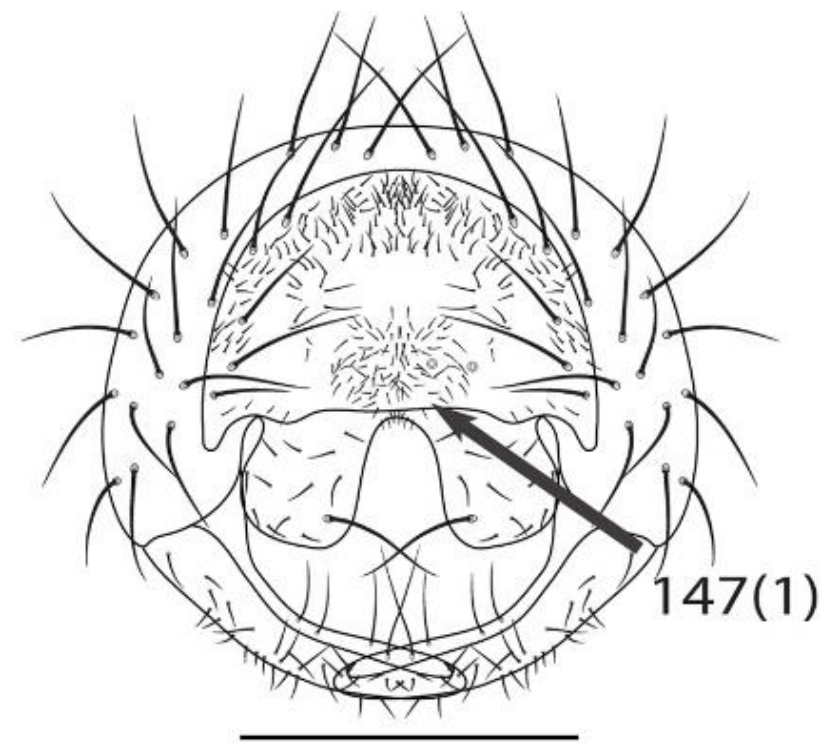

(b)

Figure 134a-b. Character 147, sub-anal plate (under proctiger): a. Hippelates plebejus, state 0 , present; b. Pseudogaurax anchora, state 1, absent.

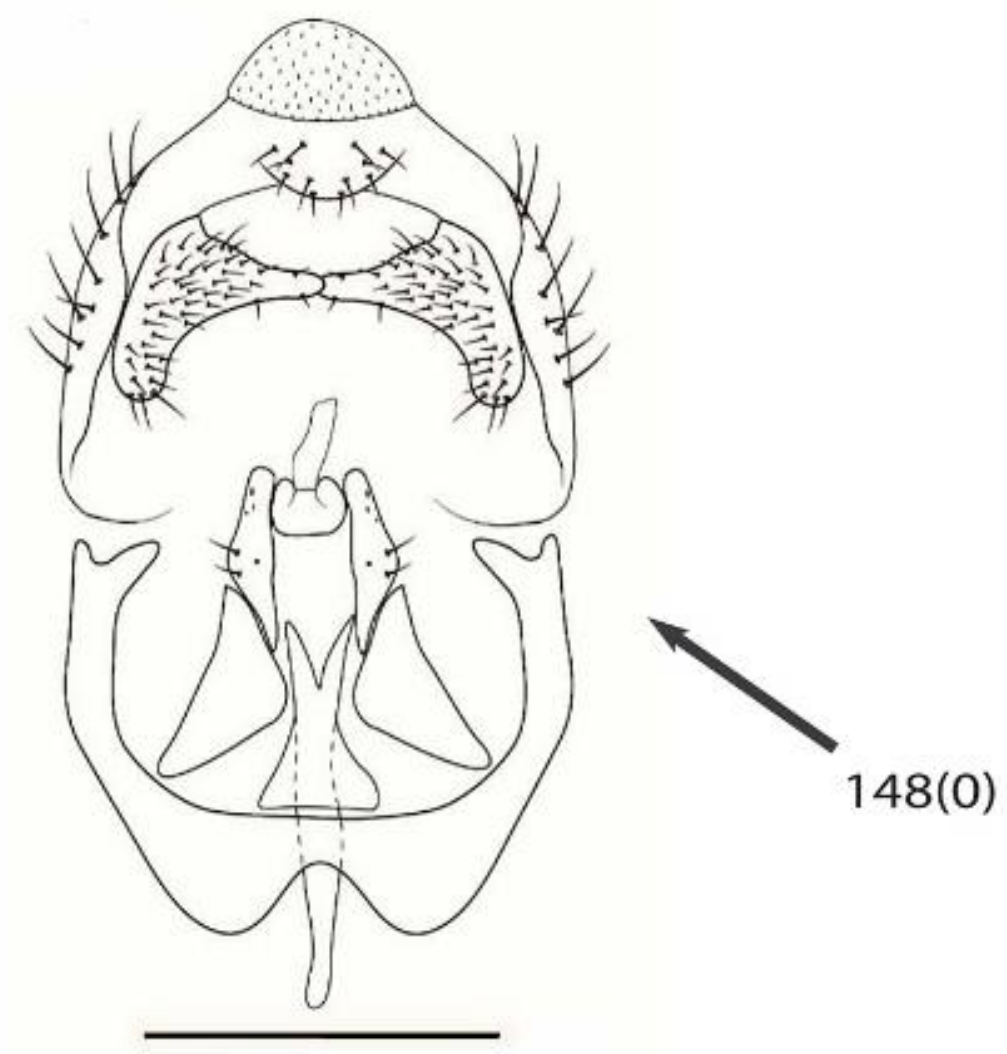

Figure 135: Character 148, hypandrium: Chloropella bipartita (Riccardi 2016), state 0, firmly connected to epandrium, articulated outside of epandrium. 


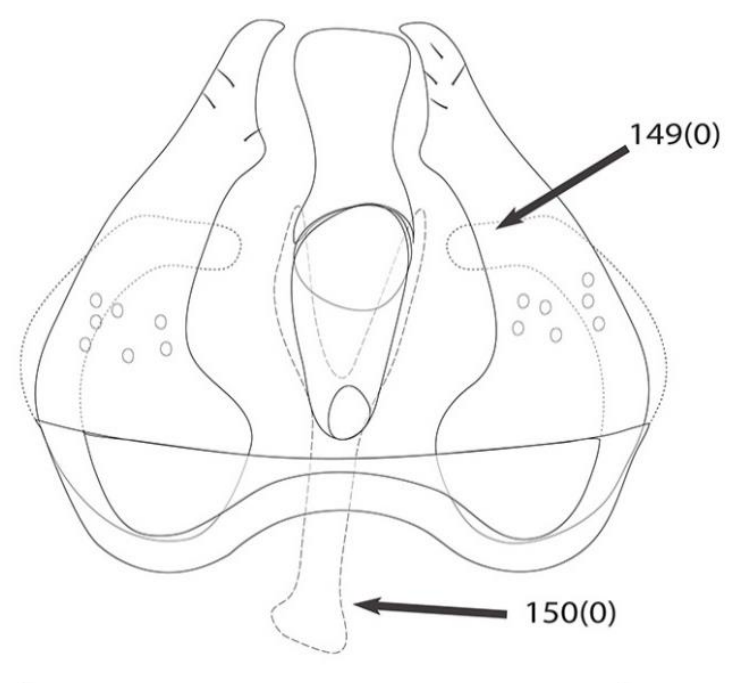

(a)

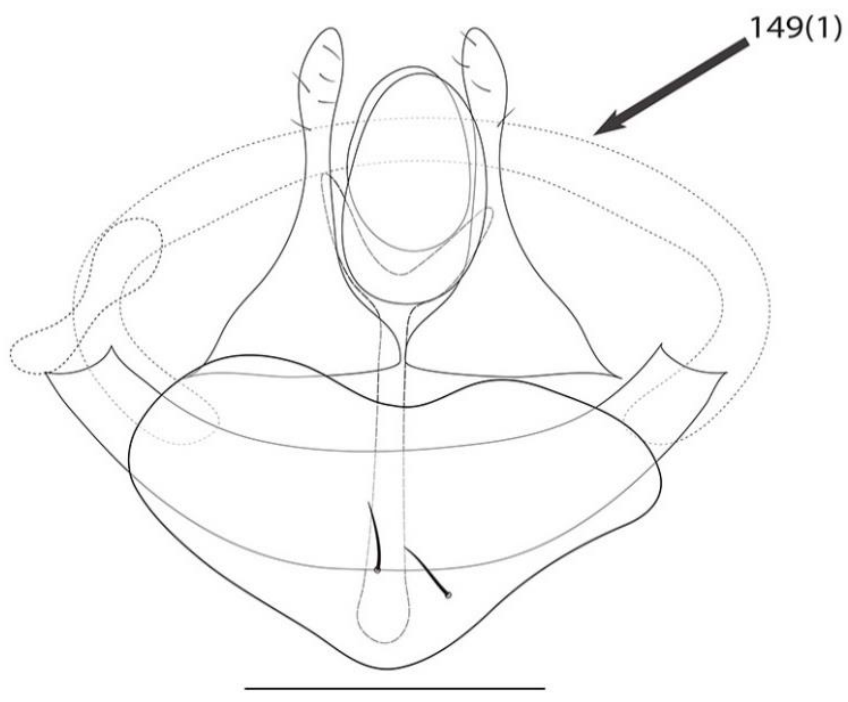

(b)

Figure 136a-b. Character 149-150; C149, shape of hypandrium; C150, phallapodeme length: a. Tricimba lineella, C149, state 0 , arms not connected posteriorly; C150, state 0 , long; b. Pseudogaurax anchora, C149, state 1, arms connected posteriorly.

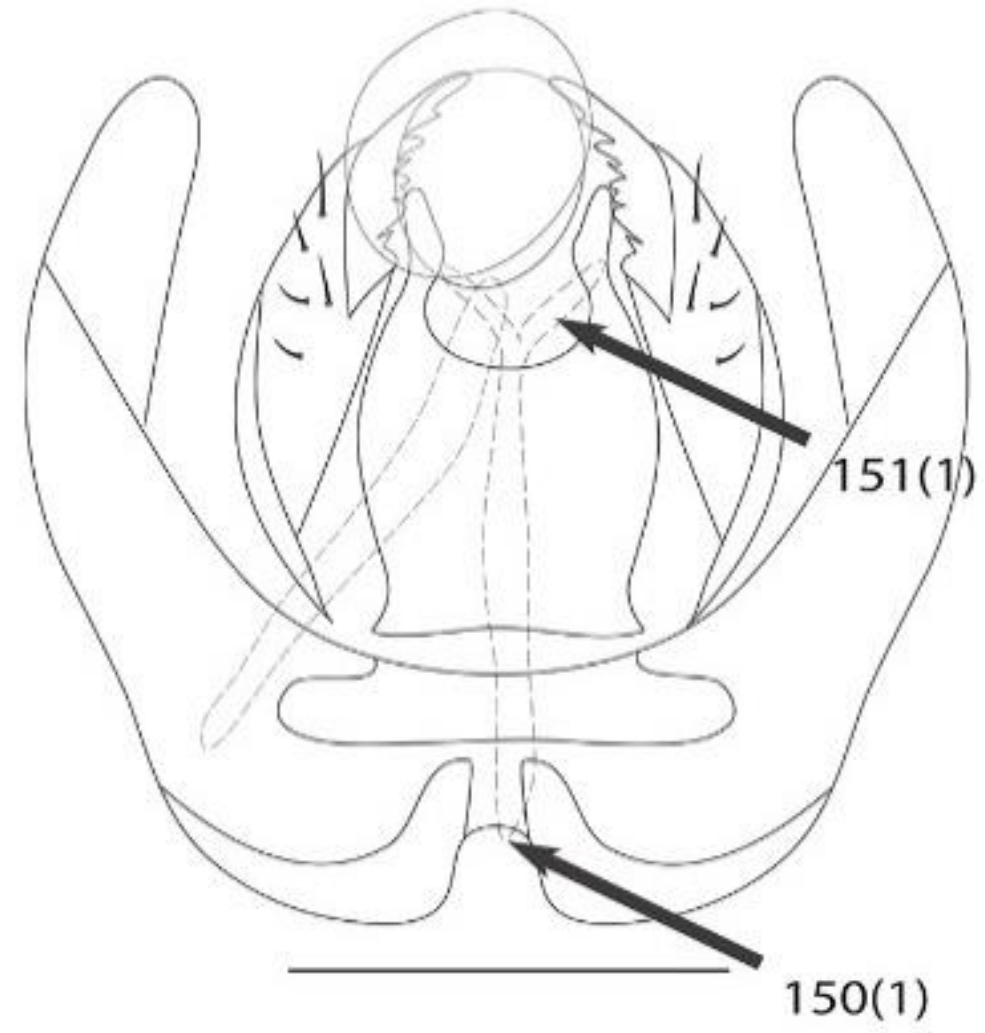

Figure 137: Character 150-151; C150, phallapodeme length and shape of base of phallapodeme; C151, base of phallapodeme: Fiebrigella sp., C150, state 1, short; C151, state 1, branched basally. 


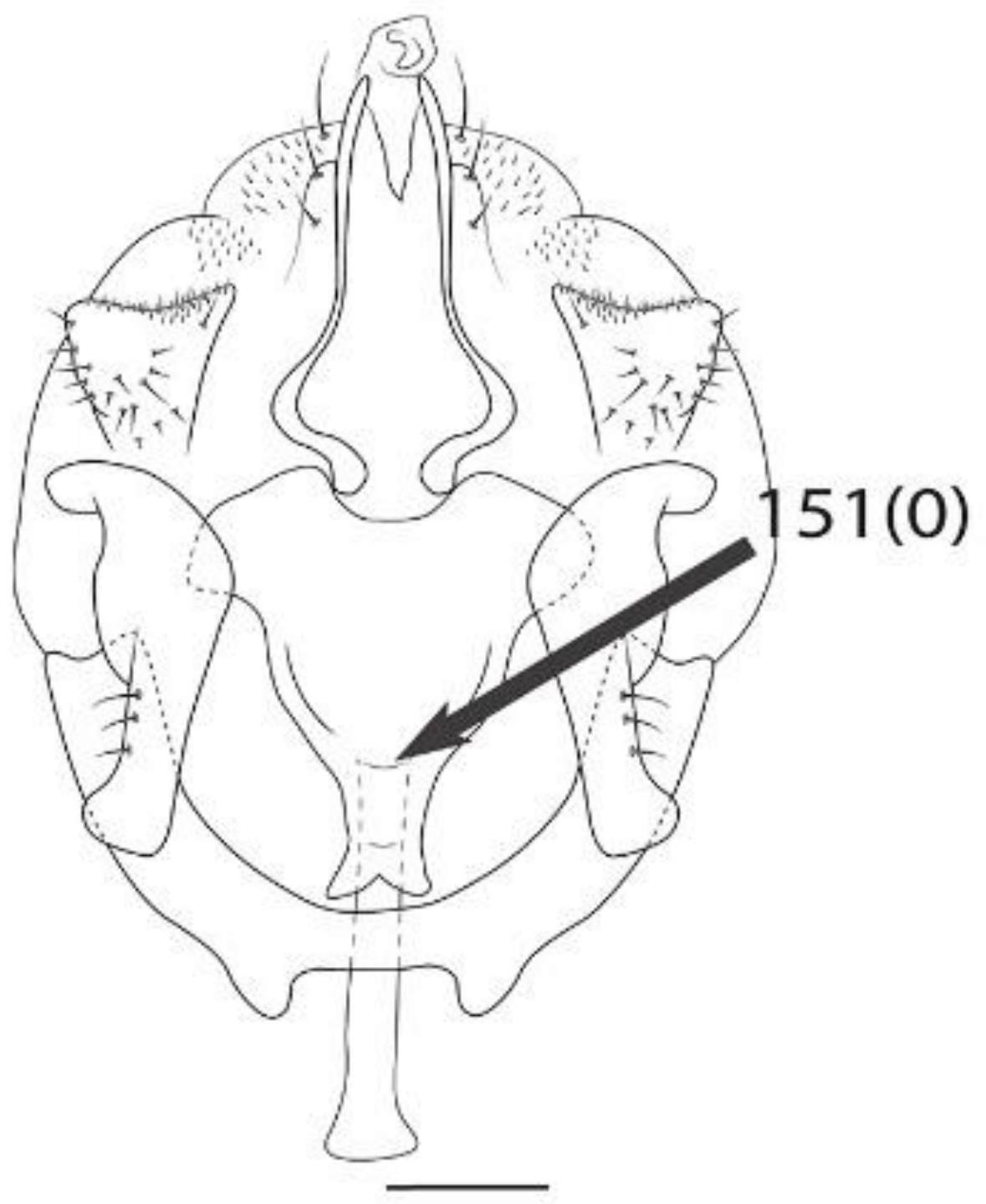

Figure 138: Character 151, base of phallapodeme: Meromyza saltatrix (Riccardi 2016), state 0 , unbranched basally. 


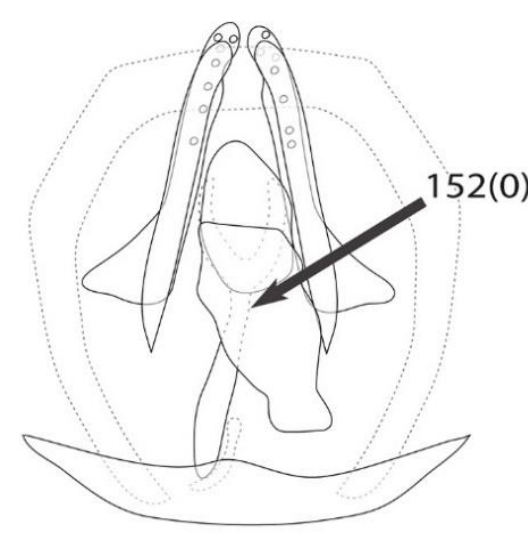

(a)

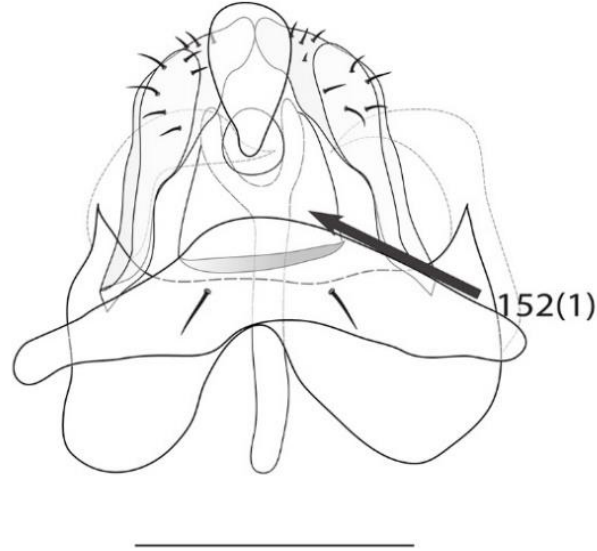

(b)

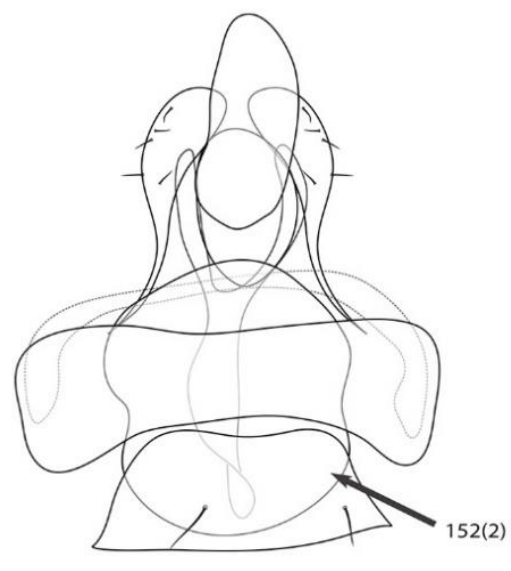

(c)

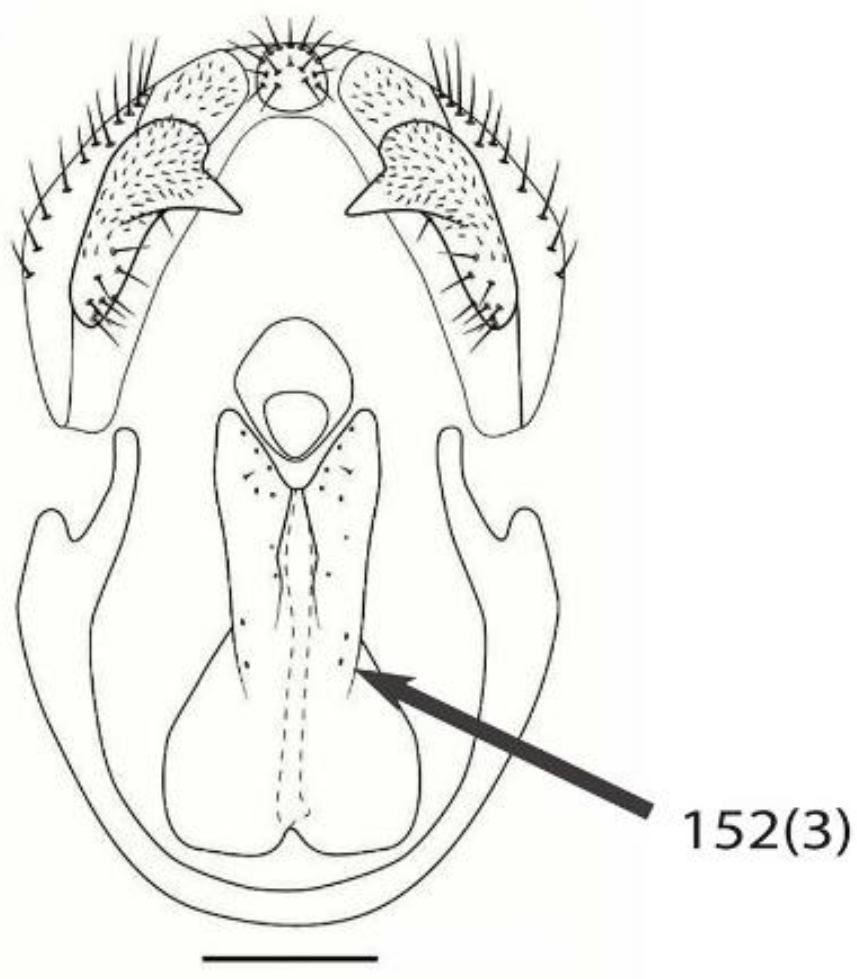

(d)

Figure 139a-d. Character 152, phallapodem sclerite: a. Apallates dissidens, state 0 , absent; b. Calamoncosis minima, state 1 , developed only basally (branches are occupied by that); $\mathrm{c}$. Notaulacella sp., state 2, developed only distally (branches are free); d. Diplotoxa versicolor (Riccardi 2016), state 3, developed basally and distally. 


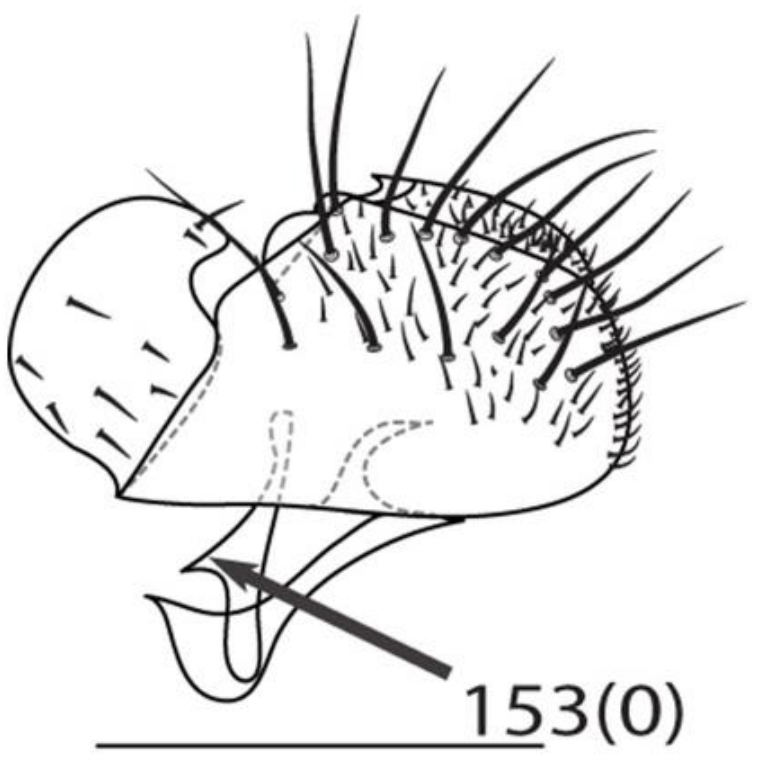

(a)

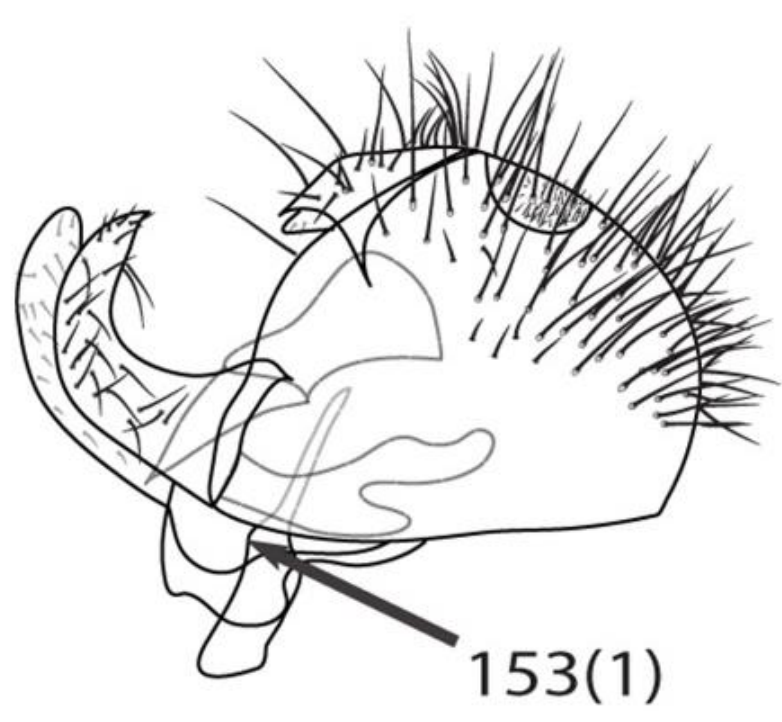

(b)

Figure 140a-b. Character 153, length of endophalic process: a. Biorbitella hesperia, state 0, developed; b. Batrachomyia nigritarsis, state 1, vestigial.

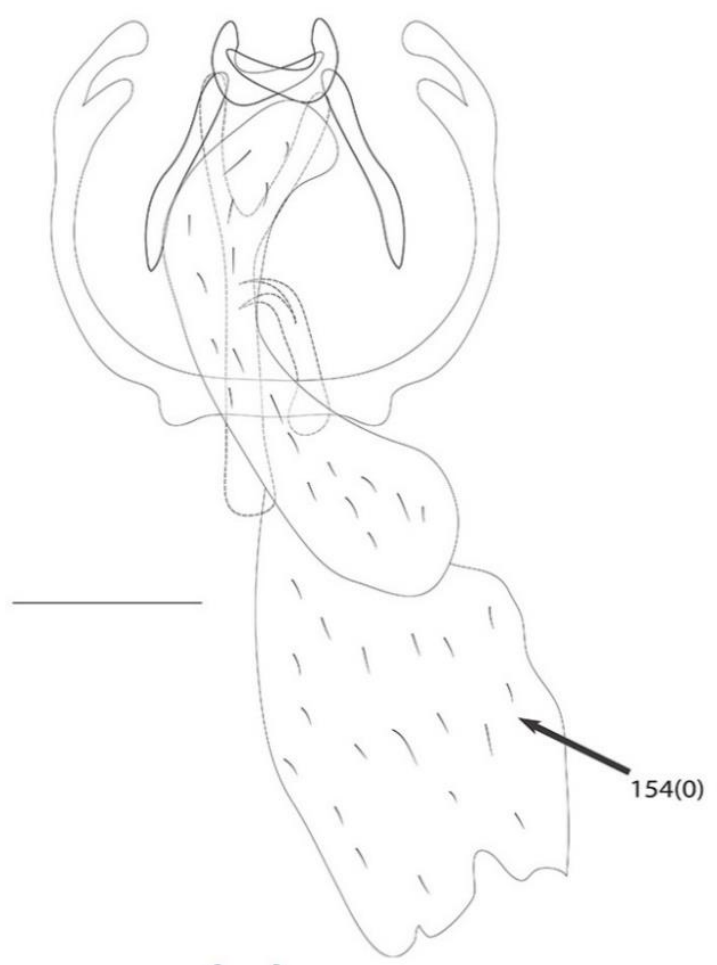

(a)

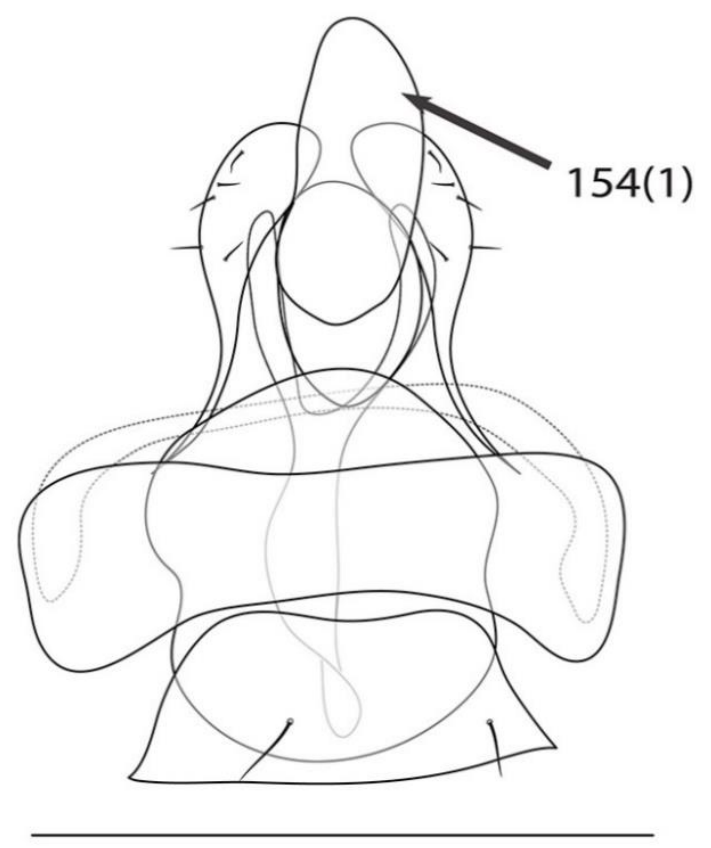

(b)

Figure 141a-b. Character 154, distiphallus: a. Meoneura flavifacies (Drawn by Paula Raile Riccardi), state 0 , hairy; b. Notaulacella sp., state 1 , bare. 


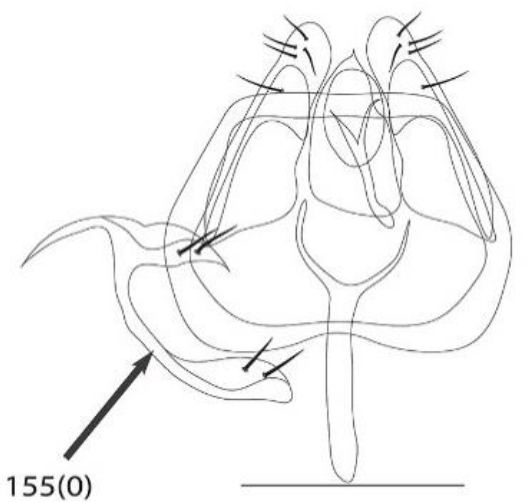

(a)

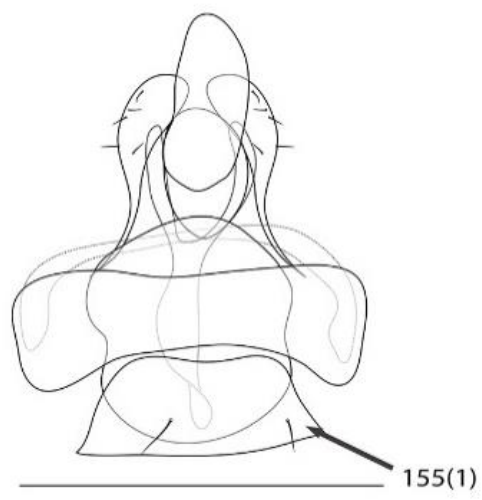

(b)

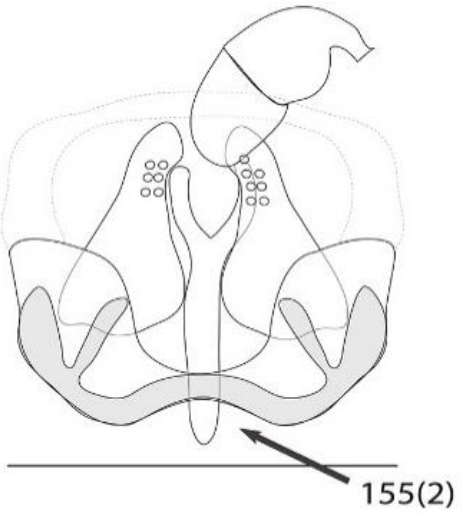

(c)

Figure 142a-c. Character 155, synchrony of S6: a. Siphonellopsis lacteibasis, state 0, asymmetrical; b. Notaulacella sp., state 1, symmetrical, frontally covers huypandrium; c. Malloewia diabolus, state 2, absent.

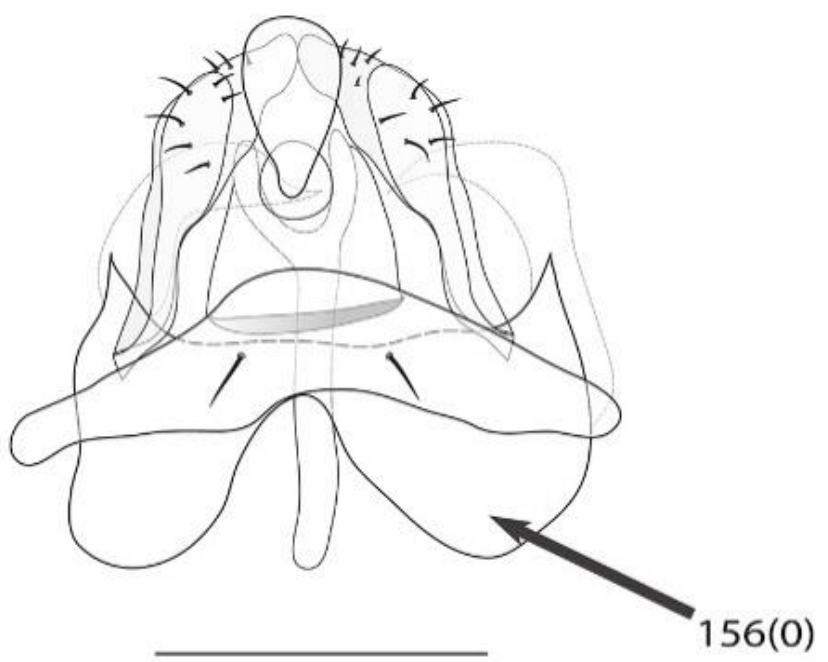

(a)

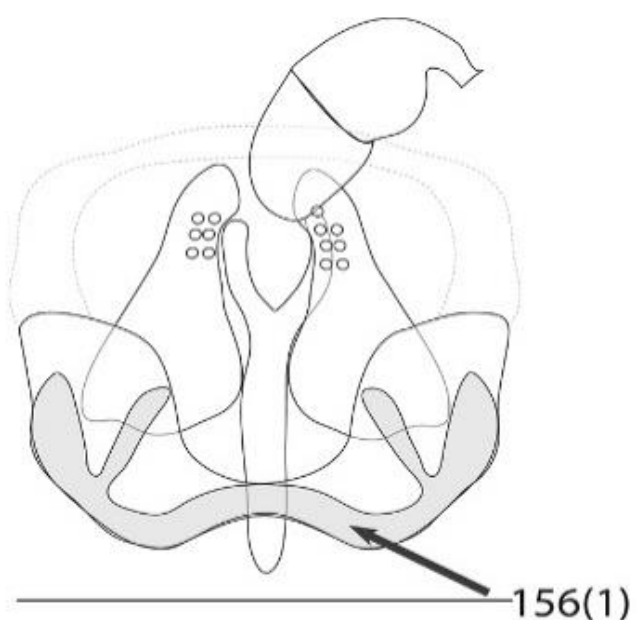

(b)

Figure 143a-b. Character 156, hypandrium, anterior view: a. Calamoncosis minima, state 0 , not sclerotized ventrally; b. Malloewia diabolus, state 1, sclerotized ventrally, divided it in 2 parts. 


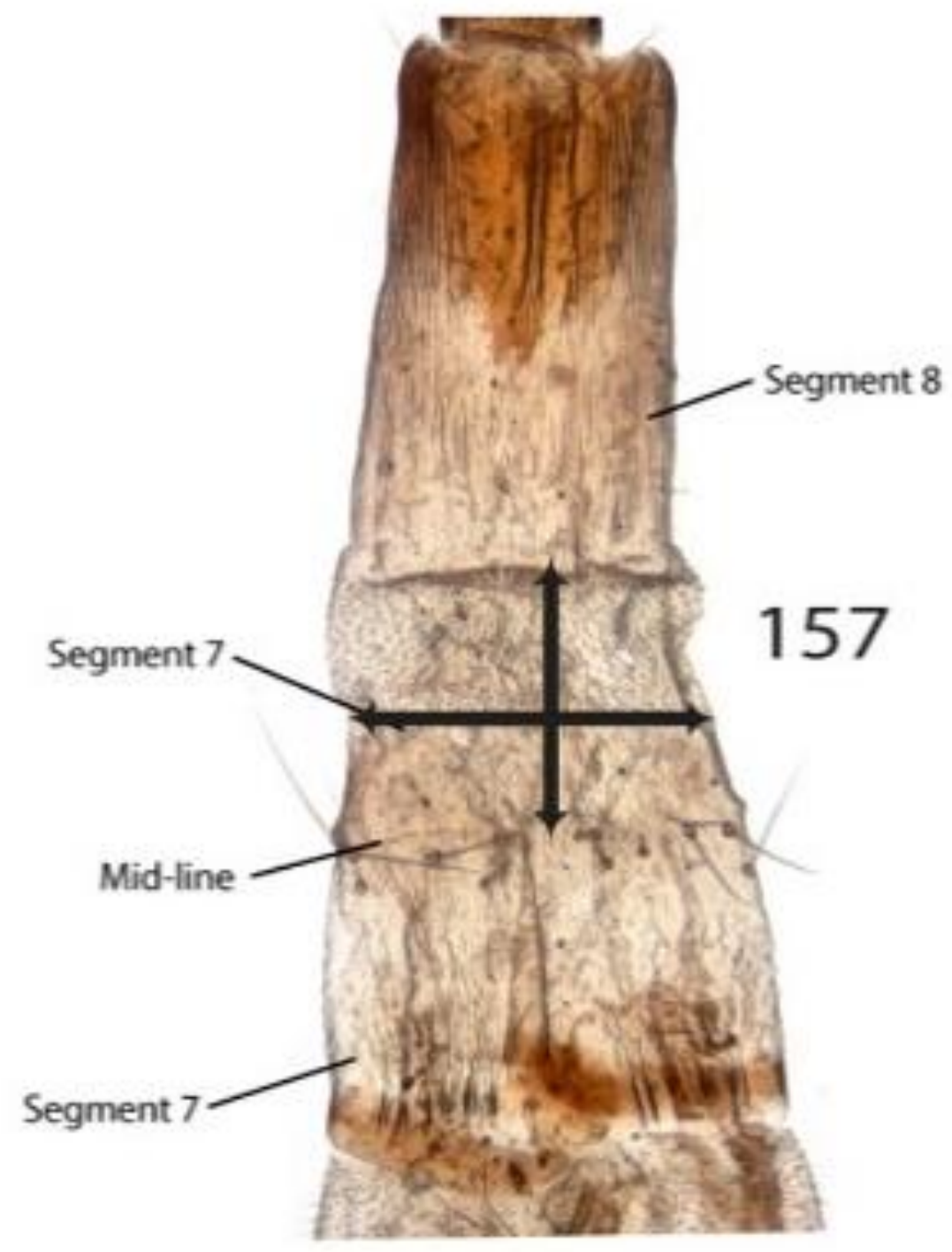

Figure 144: Character 157, shape of segment 7: Anatrichus erinaceus. 


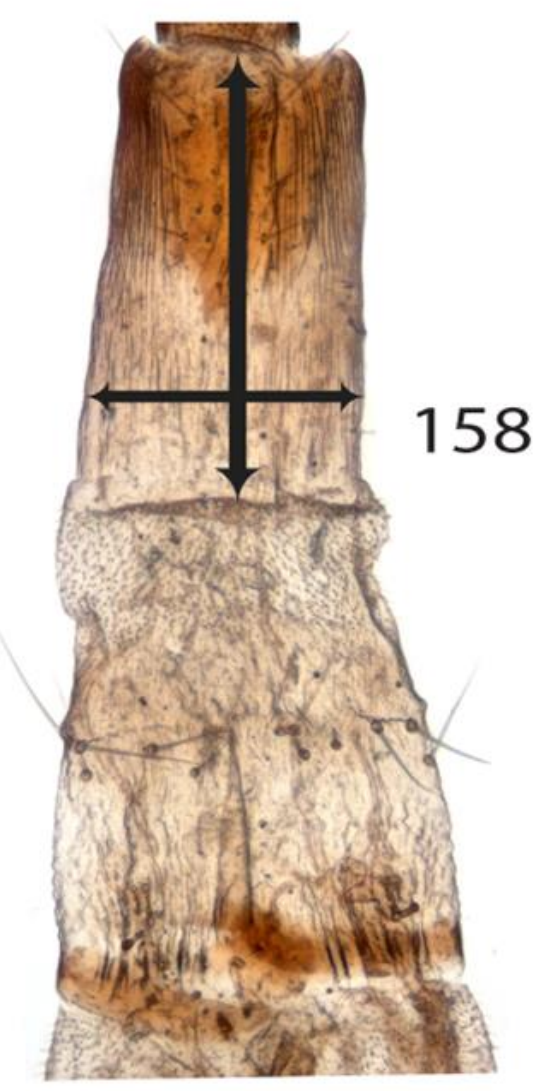

Figure 145: Character 158, shape of segment 8: Anatrichus erinaceus.

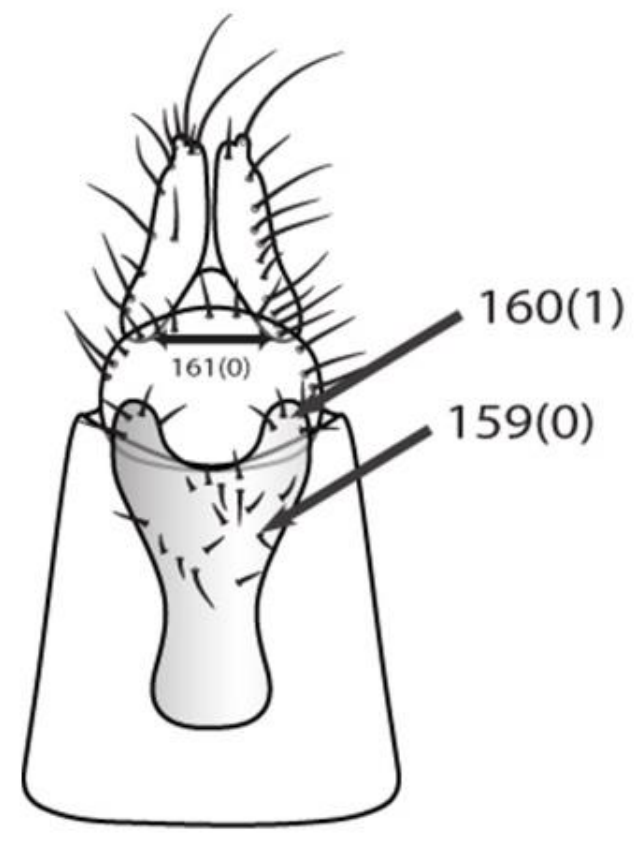

(a)

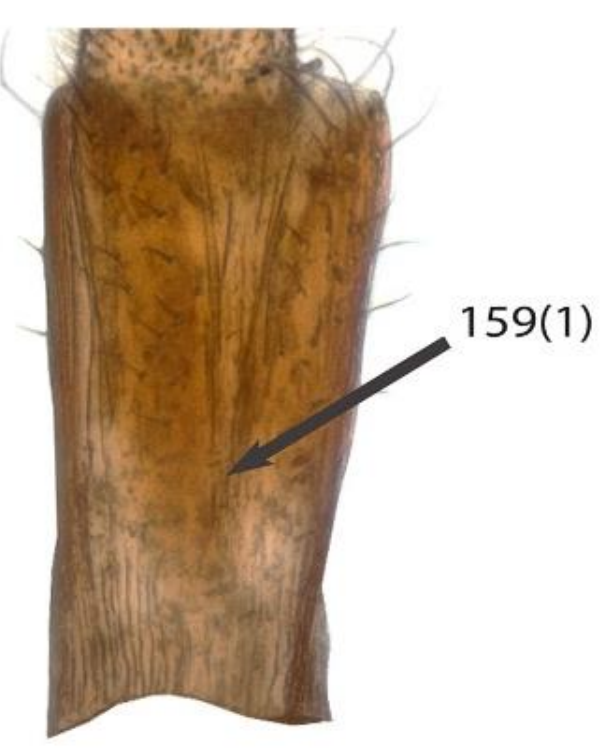

(b)

Figure 146a-b. Characters 159-160; C159, sternite 8; C160, shape of sternite 8: a. Schematic shape, C159, state 0 , one piece; $\mathrm{C} 160$, state 1 , protruding in 2 lobes anteriorly; b. Disciphus peregrinus, C159, state 1, V-shaped. 


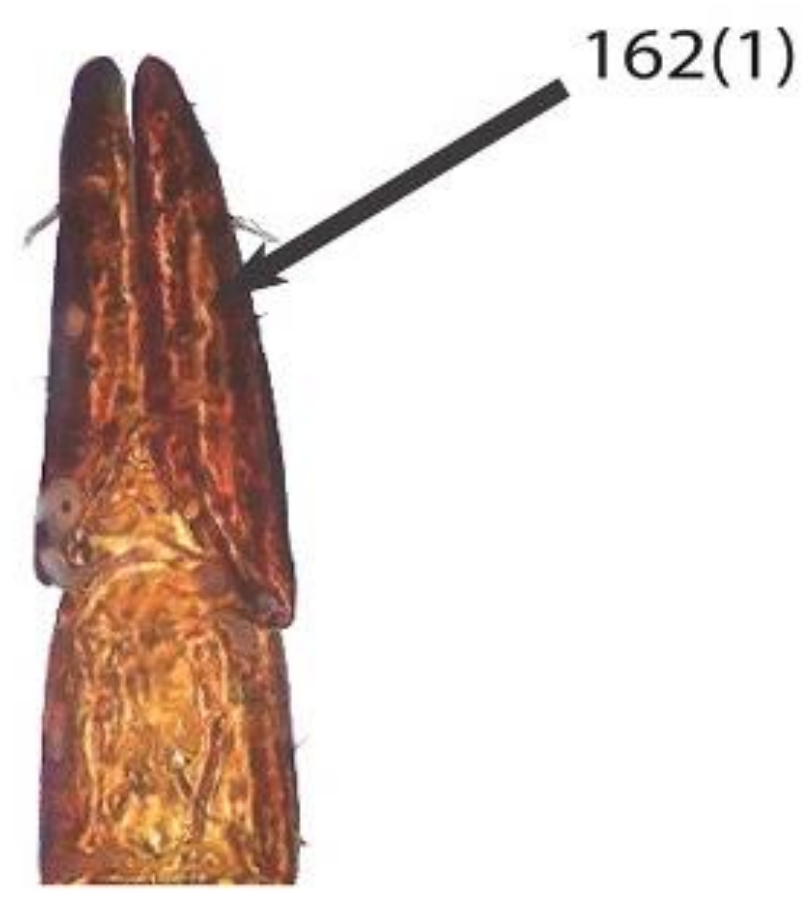

Figure 147: Character 162, cerci: Opetiophora straminea, state 1, sclerotized.

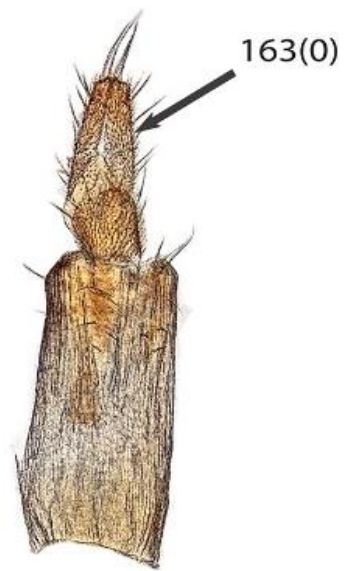

(a)

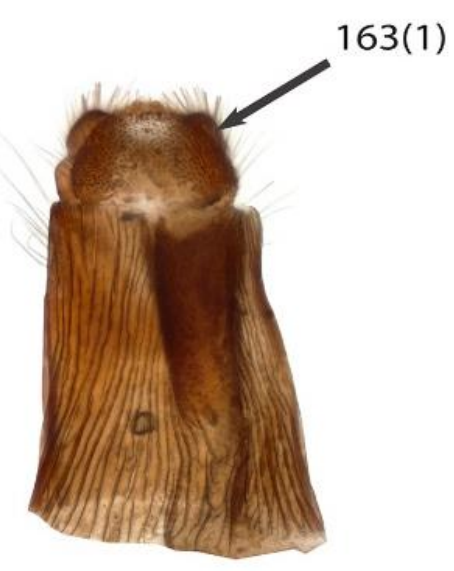

(b)

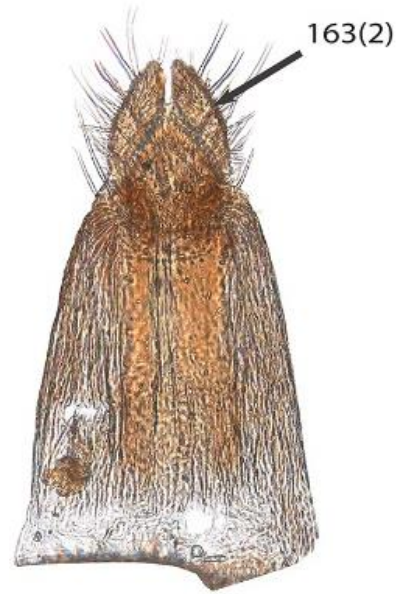

(c)

Figure 148a-c. Character 163, shape of cerci: a. Oscinimorpha albisetosa, state 0 , elongated, cylindral; b. Lipara lucens, state 1, short, round; c. Speccafrons mallochi, state 2, short, triangular. 


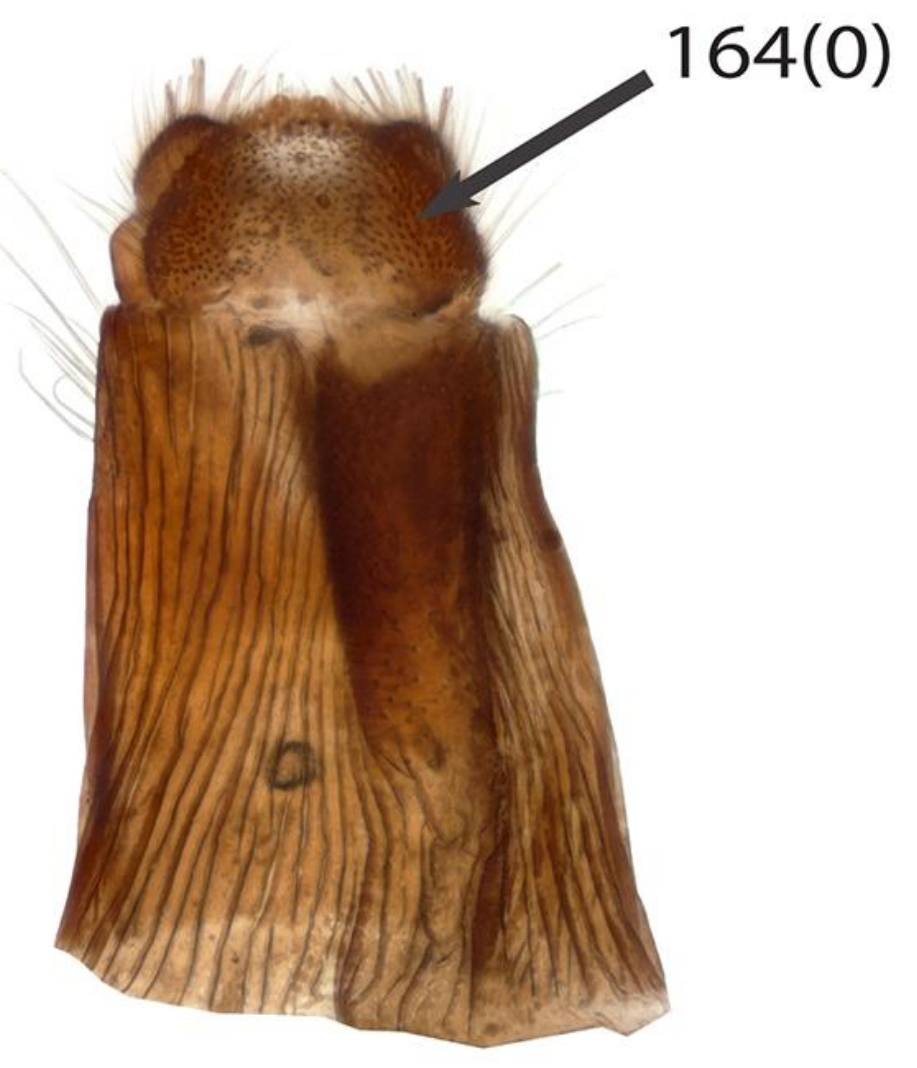

Figure 149: Character 164, hypoproct: Lipara lucens, state 0 , densely covered with microtrichia.

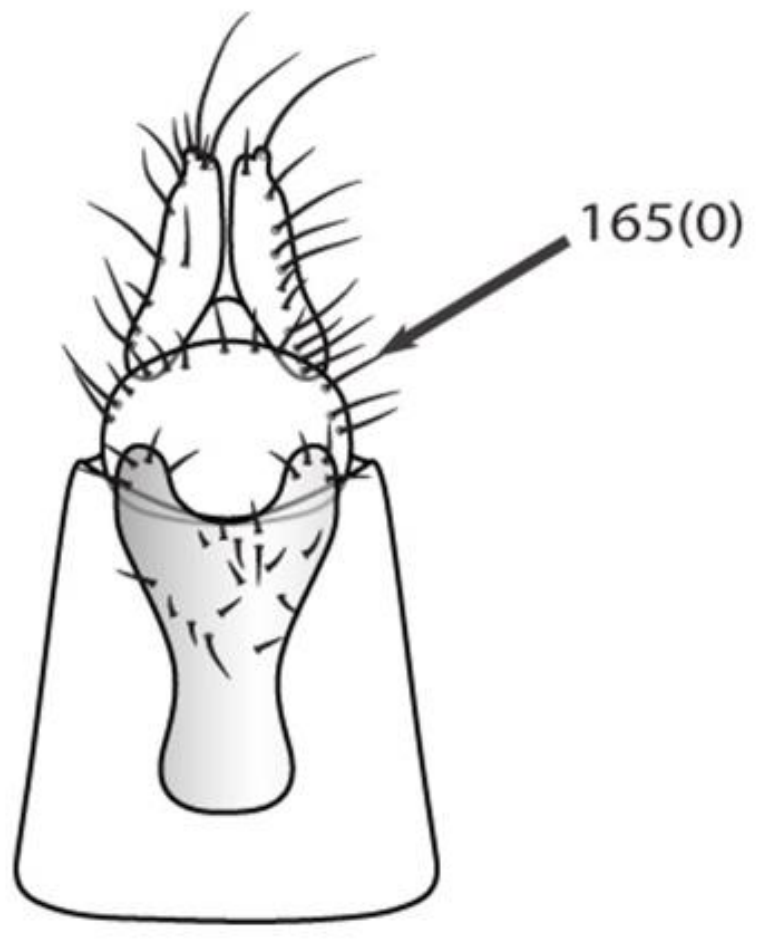

Figure 150: Character 165, hypoproct setation: Schematic shape, state 0, aligned at the frontal margin of hypandrium in row(s). 


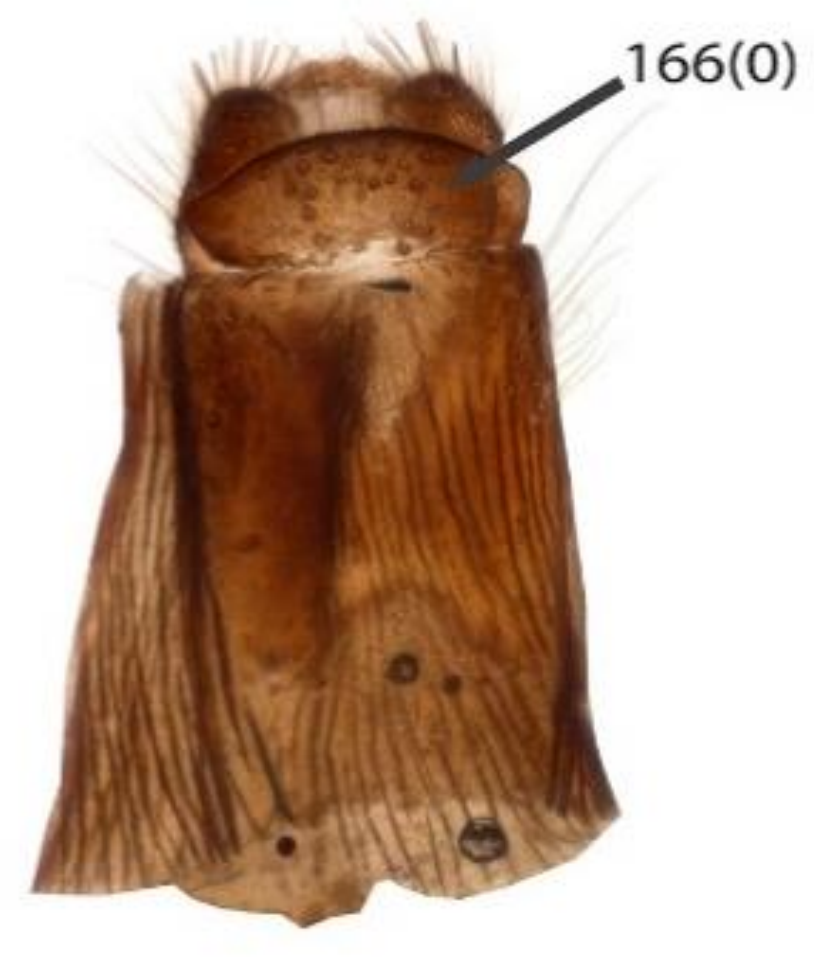

Figure 151: Character 166, epiproct: Lipara lucens, state 0, with microtrichia.

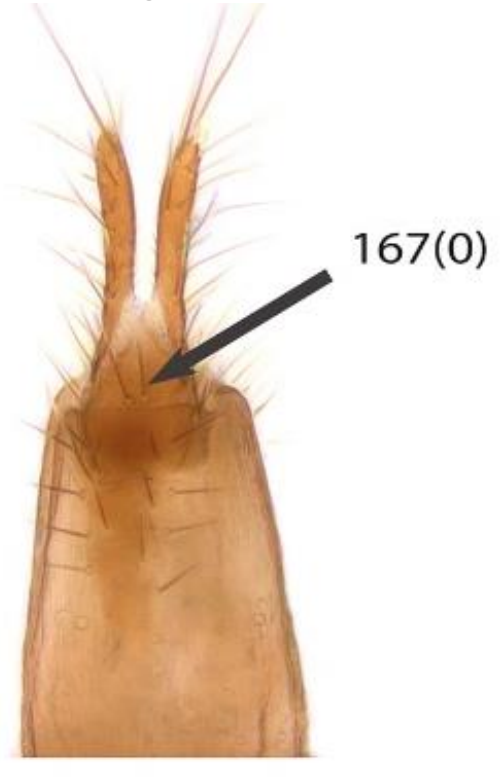

(a)

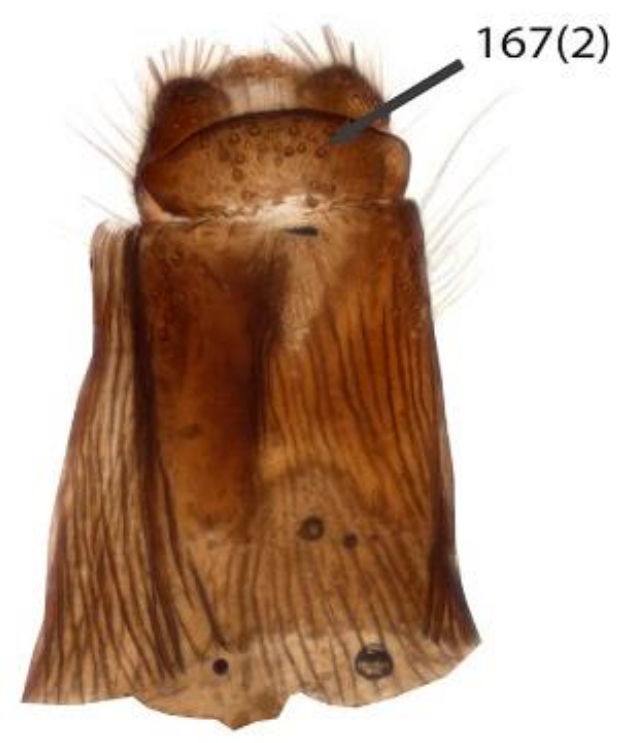

(b)

Figure 152a-b. Character 167, number of setae on epiproct: a. Psilacrum lineatum (Riccardi \& Ismay 2017), state 0 , two in the centre of epiproct disk; b. Lipara lucens, state 2, numerous and dispersal. 
Table 
Table 1: Morphological data matrix. ?, missing data; -, inapplicable characters

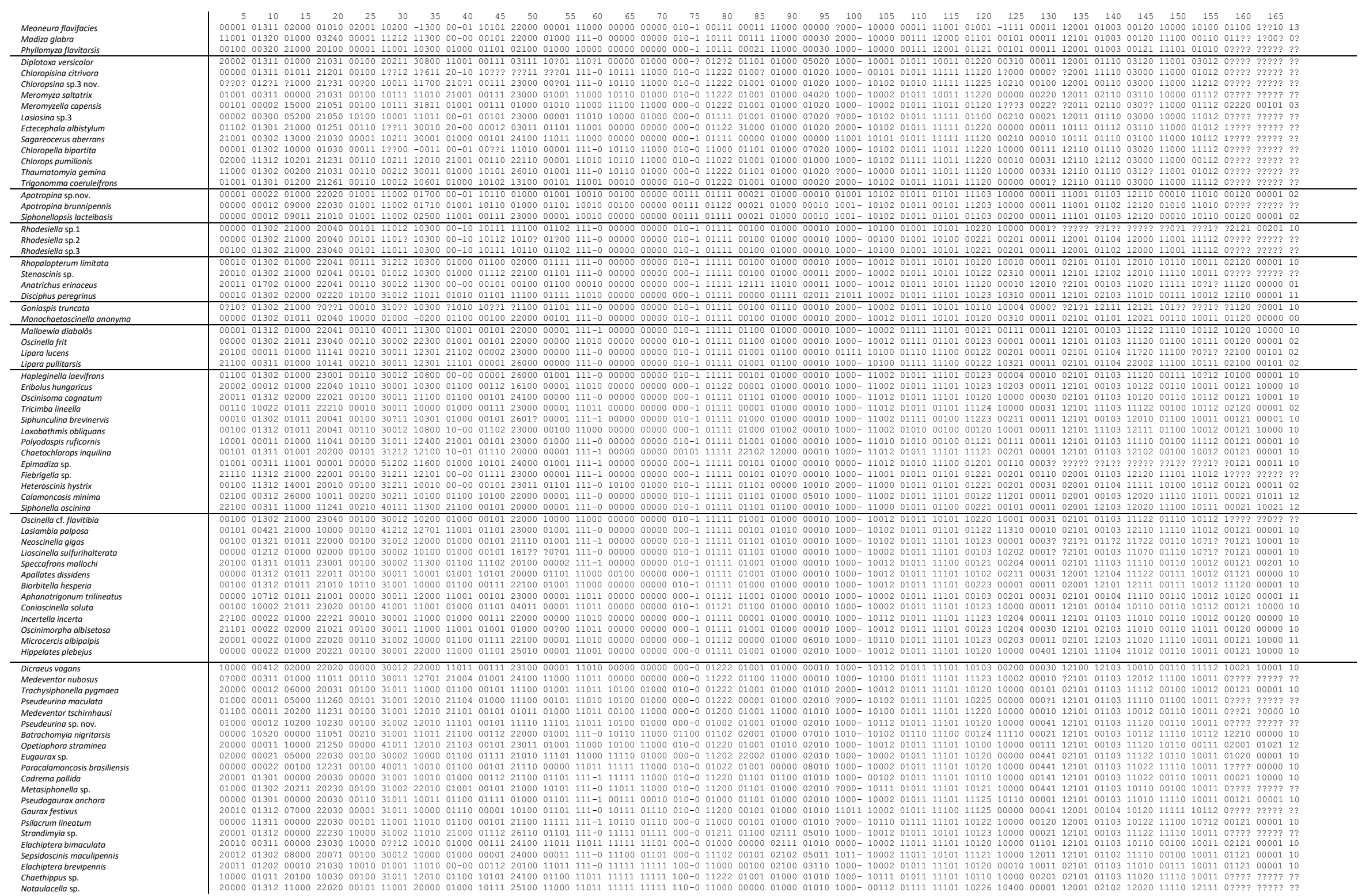


Appendix 
Appendix I. Placement of the genera of Oscinellinae and Rhodesiellinae into tribes or genus groups in different papers.

\begin{tabular}{|c|c|c|c|c|c|}
\hline Genus & Andersson 1977 & Kanmiya 1983 & Nartshuk 1983 & Nartshuk 2012 & $\begin{array}{c}\text { Mlynarek \& } \\
\text { Wheeler } 2018\end{array}$ \\
\hline Acanthopeltastes Enderlein, 1911 & Unplaced & Unplaced & Unplaced & Fiebrigellini & Unplaced \\
\hline $\begin{array}{l}\text { Agrophaspidium Wheeler \& Mlynarek, } \\
2008\end{array}$ & Unplaced & Unplaced & Unplaced & Unplaced & Unplaced \\
\hline Allomedeia Mlynarek \& Wheeler, 2010 & Unplaced & Unplaced & Unplaced & Unplaced & Elachipterini \\
\hline Alombus Becker, 1914 & Unplaced & Unplaced & Elachipterini & Elachipterini & Elachipterini \\
\hline Amnonella Cherian, 1992 & Unplaced & Unplaced & Unplaced & Rhodesiellini & Unplaced \\
\hline Anacamptoneurum Becker, 1903 & Unplaced & Polyodaspis (Oscinellini s.I.) & Fiebrigellini & Unplaced & Unplaced \\
\hline Anatrichus Loew, 1860 & Elachiptera (Oscinellini s.I.) & Elachiptera (Oscinellini s.I.) & Elachipterini & Elachipterini & Elachipterini \\
\hline Anomoeoceros Lamb, 1918 & Lipara (Oscinellini s.I.) & Lipara (Oscinellini s.I.) & Siphonellini & Liparaini & Unplaced \\
\hline Apallates Sabrosky, 1980 & Unplaced & Unplaced & Incertellini & Incertellini & Unplaced \\
\hline Aphanotrigonella Nartshuk, 1964 & $\begin{array}{l}\text { Aphanotrigonum (Oscinellini } \\
\text { s.I.) }\end{array}$ & Unplaced & Tricimbini & Tricimbini & Unplaced \\
\hline Aphanotrigonum Duda, 1932 & $\begin{array}{l}\text { Aphanotrigonum (Oscinellini } \\
\text { s.l.) }\end{array}$ & $\begin{array}{l}\text { Aphanotrigonum (Oscinellini } \\
\text { s.I.) }\end{array}$ & Incertellini & Incertellini & Unplaced \\
\hline Aprometopis Becker, 1910 & $\begin{array}{l}\text { Aphanotrigonum (Oscinellini } \\
\text { s.l.) }\end{array}$ & $\begin{array}{l}\text { Aphanotrigonum (Oscinellini } \\
\text { s.I.) }\end{array}$ & Unplaced & Tricimbini & Unplaced \\
\hline Apterosepsis Richards, 1962 & Unplaced & Unplaced & Unplaced & Unplaced & Unplaced \\
\hline Arcuator Sabrosky, 1985 & Unplaced & Unplaced & Unplaced & Unplaced & Unplaced \\
\hline Aulacogaurax Becker \& Meijere, 1913 & Unplaced & Unplaced & Unplaced & Unplaced & Unplaced \\
\hline Batrachomyia Krefft, 1864 & Unplaced & Unplaced & Unplaced & Unplaced & Unplaced \\
\hline Benjaminella Malloch, 1925 & Unplaced & Unplaced & Unplaced & Unplaced & Unplaced \\
\hline Bharathella Cherian, 1990 & Unplaced & Unplaced & Unplaced & Rhodesiellini & Unplaced \\
\hline Biorbitella Sabrosky, 1980 & Unplaced & Unplaced & Incertellini & Incertellini & Unplaced \\
\hline Cadrema Walker, 1859 & Elachiptera (Oscinellini s.I.) & Cadrema (Oscinellini s.I.) & Hippelatinae s.I. & Unplaced & Unplaced \\
\hline Calamoncosis Enderlein, 1911 & Lipara (Oscinellini s.I.) & Lipara (Oscinellini s.I.) & Siphonellini & Liparaini & Unplaced \\
\hline
\end{tabular}




\begin{tabular}{|c|c|c|c|c|c|}
\hline $\begin{array}{l}\text { Camptopeltes Wheeler \& Mlynarek, } \\
2010\end{array}$ & Unplaced & Unplaced & Unplaced & Unplaced & Unplaced \\
\hline Camptoscinella Sabrosky, 1951 & Unplaced & Unplaced & Oscinellini & Oscinellini & Unplaced \\
\hline Cauloscinis Yang \& Yang, 1991 & Unplaced & Unplaced & Unplaced & Unplaced & Unplaced \\
\hline Caviceps Malloch, 1924 & Unplaced & $\begin{array}{l}\text { Aphanotrigonum (Oscinellini } \\
\text { s.l.) }\end{array}$ & Unplaced & Unplaced & Unplaced \\
\hline Cestoplectus Lamb, 1918 & Gaurax (Oscinellini s.I.) & Gaurax (Oscinellini s.I.) & Unplaced & Botanobiini & Unplaced \\
\hline Chaethippus Duda, 1930 & Unplaced & Unplaced & Unplaced & Unplaced & Unplaced \\
\hline Chaetochlorops Malloch, 1914 & Unplaced & Polyodaspis (Oscinellini s.I.) & Fiebrigellini & Fiebrigellini & Unplaced \\
\hline Conioscinella Duda, 1929 & $\begin{array}{l}\text { Aphanotrigonum (Oscinellini } \\
\text { s.l.) }\end{array}$ & $\begin{array}{l}\text { Aphanotrigonum (Oscinellini } \\
\text { s.I.) }\end{array}$ & Oscinellini & Oscinellini & Unplaced \\
\hline Coryphisoptron Enderlein, 1911 & Unplaced & Unplaced & Unplaced & Unplaced & Unplaced \\
\hline Cyrtomomyia Becker, 1913 & Unplaced & Unplaced & Elachipterini & Elachipterini & $\begin{array}{l}\text { Elachipterini } \\
\text { (Synonimyzed) }\end{array}$ \\
\hline Dactylothyrea Meijere, 1910 & Rhodesiellini s.l. & Rhodesiellini s.I. & Rhodesiellini & Rhodesiellini & Unplaced \\
\hline Dasyopa Malloch, 1918 & Unplaced & Unplaced & Siphonellini & Liparaini & Unplaced \\
\hline Deltastoma Malloch, 1924 & Unplaced & Unplaced & Unplaced & Unplaced & Unplaced \\
\hline Dicraeus Loew, 1873 & Dicreaus (Oscinellini s.I.) & Dicraeus (Oscinellini s.I.) & Dicraeini & Dicraeini & Unplaced \\
\hline Disciphus Becker, 1911 & Elachiptera (Oscinellini s.I.) & Elachiptera (Oscinellini s.I.) & Elachipterini & Elachipterini & Elachipterini \\
\hline Discogastrella Enderlein, 1911 & Unplaced & Unplaced & Unplaced & Unplaced & Unplaced \\
\hline Dysartia Sabrosky, 1991 & Unplaced & Unplaced & Unplaced & Unplaced & Unplaced \\
\hline Elachiptera Macquart, 1835 & Elachiptera (Oscinellini s.I.) & Elachiptera (Oscinellini s.I.) & Elachipterini & Elachipterini & Elachipterini \\
\hline Elaphaspis Bezzi, 1912 & Unplaced & Unplaced & Unplaced & Unplaced & Unplaced \\
\hline Enderleiniella Becker, 1912 & Unplaced & Unplaced & Unplaced & Unplaced & Unplaced \\
\hline Epimadiza Becker, 1910 & Unplaced & Polyodaspis (Oscinellini s.l.) & Fiebrigellini & Fiebrigellini & Unplaced \\
\hline Eribolus Becker, 1910 & Eribolus (Oscinellini s.l.) & Unplaced & Oscinisomatini & Oscinisomatini & Unplaced \\
\hline Eugaurax Malloch, 1913 & Unplaced & Unplaced & Unplaced & Unplaced & Unplaced \\
\hline Euthyridium Frey, 1923 & Rhodesiellini s.I. & Rhodesiellini s.l. & Rhodesiellini & Rhodesiellini & Unplaced \\
\hline
\end{tabular}




\begin{tabular}{|c|c|c|c|c|c|}
\hline Fiebrigella Duda, 1921 & Polyodaspis (Oscinellini s.I.) & Polyodaspis (Oscinellini s.I.) & Fiebrigellini & Fiebrigellini & Unplaced \\
\hline Gampsocera Schiner, 1862 & Gaurax (Oscinellini s.I.) & Gaurax (Oscinellini s.I.) & Botanobiini & Botanobiini & Unplaced \\
\hline Gaurax Loew, 1863 & Gaurax (Oscinellini s.I.) & Gaurax (Oscinellini s.I.) & Botanobiini & Botanobiini & Unplaced \\
\hline Goniaspis Duda, 1930 & Unplaced & Unplaced & Unplaced & Unplaced & Elachipterini \\
\hline Hapleginella Duda, 1933 & Oscinella (Oscinellini s.I.) & Oscinella (Oscinellini s.I.) & Botanobiini & Botanobiini & Unplaced \\
\hline Heteroscinis Lamb, 1918 & Unplaced & Unplaced & Unplaced & Fiebrigellini & Unplaced \\
\hline Heteroscinoides Cherian, 1989 & Unplaced & Unplaced & Fiebrigellini & Fiebrigellini & Unplaced \\
\hline Hippelates Loew, 1863 & Unplaced & Unplaced & Hippelatinae s.I. & Unplaced & Unplaced \\
\hline Incertella Sabrosky, 1980 & Unplaced & Oscinella (Oscinellini s.I.)?? & Incertellini & Incertellini & Unplaced \\
\hline Indometopis Cherian, 1989 & Unplaced & Unplaced & Unplaced & Tricimbini & Unplaced \\
\hline $\begin{array}{l}\text { Indonella Cherian in Cherian \& } \\
\text { Shinimol, } 2008\end{array}$ & Unplaced & Unplaced & Unplaced & Unplaced & Unplaced \\
\hline Indophthalmus Cherian, 2002 & Unplaced & Unplaced & Unplaced & Rhodesiellini & Unplaced \\
\hline Javanoscinis Andersson, 1977 & Javanoscinis (Oscinellini s.I.) & Unplaced (Oscinellini s.I.) & Stenoscinini & Stenoscinini & Unplaced \\
\hline Kurumemyia Kanmiya, 1983 & Unplaced & Siphonella (Oscinellini s.I.) & Unplaced & Siphonellini & Unplaced \\
\hline Kwarea Sabrosky, 1954 & Unplaced & Unplaced & Unplaced & Unplaced & Unplaced \\
\hline Lasiambia Anonymous & Polyodaspis (Oscinellini s.I.) & Polyodaspis (Oscinellini s.I.) & Fiebrigellini & Fiebrigellini & Unplaced \\
\hline Lasiochaeta Corti, 1909 & Unplaced & Unplaced & Unplaced & Elachipterini & \begin{tabular}{|l} 
Elachipterini \\
(Synonimyzed)
\end{tabular} \\
\hline Leucochaeta Becker, 1913 & Unplaced & Unplaced & Botanobiini & Botanobiini & Unplaced \\
\hline Liohippelates Duda 1929 & Unplaced & Unplaced & Hippelatinae s.l. & Unplaced & Unplaced \\
\hline Lioscinella Duda 1929 & Oscinella (Oscinellini s.I.) & Oscinella (Oscinellini s.I.)?? & Hippelatinae s.I. & Unplaced & Unplaced \\
\hline Lipara Meigen, 1830 & Lipara (Oscinellini s.I.) & Lipara (Oscinellini s.I.) & Siphonellini & Liparaini & Unplaced \\
\hline Loxobathmis Enderlein, 1911 & Unplaced & Unplaced & Unplaced & Unplaced & Unplaced \\
\hline Malloewia Sabrosky, 1980 & Unplaced & Unplaced & Incertellini & Incertellini & Unplaced \\
\hline Medeventor Wheeler, 2007 & Unplaced & Unplaced & Unplaced & Unplaced & Unplaced \\
\hline
\end{tabular}




\begin{tabular}{|c|c|c|c|c|c|}
\hline Meijerella Sabrosky, 1976 & Unplaced & $\begin{array}{l}\text { Aphanotrigonum (Oscinellini } \\
\text { s.l.) }\end{array}$ & Unplaced & Incertellini & Unplaced \\
\hline Melanochaetomyia Cherian, 2002 & Unplaced & Unplaced & Unplaced & Elachipterini & Unplaced \\
\hline Merobates Duda, 1934 & Unplaced & Unplaced & Rhodesiellini & Unplaced & Unplaced \\
\hline Merodonta Malloch, 1940 & Unplaced & Unplaced & Unplaced & Unplaced & Unplaced \\
\hline Metasiphonella Duda, 1930 & Unplaced & Unplaced & Unplaced & Unplaced & Unplaced \\
\hline Microcercis Beschovski, 1978 & Unplaced & Unplaced & Unplaced & Incertellini & Unplaced \\
\hline Mimosepsis Sabrosky, 1951 & Unplaced & Unplaced & Unplaced & Unplaced & Unplaced \\
\hline Monochaetoscinella Duda, 1930 & Unplaced & Unplaced & Unplaced & Unplaced & Unplaced \\
\hline Neolcella Cherian, 1996 & Unplaced & Unplaced & Unplaced & Oscinellini & Unplaced \\
\hline Neorhodesiella Cherian, 2002 & Unplaced & Unplaced & Unplaced & Rhodesiellini & Unplaced \\
\hline Neoscinella Sabrosky, 1980 & Unplaced & Unplaced & Unplaced & Oscinellini & Unplaced \\
\hline Nomba Walker, 1860 & Rhodesiellini s.I. & Rhodesiellini s.I. & Unplaced & Rhodesiellini & Unplaced \\
\hline Notaulacella Enderlein, 1911 & Unplaced & Unplaced & Unplaced & Unplaced & Unplaced \\
\hline Olcella Enderlein, 1911 & Unplaced & Unplaced & Oscinellini & Oscinellini & Unplaced \\
\hline Onychaspidium Enderlein, 1911 & Unplaced & Unplaced & Unplaced & Unplaced & Unplaced \\
\hline Opetiophora Loew, 1872 & Unplaced & Unplaced & Unplaced & Unplaced & Unplaced \\
\hline Oscinella Becker, 1909 & Oscinella (Oscinellini s.I.) & Oscinella (Oscinellini s.I.) & Oscinellini & Oscinellini & Unplaced \\
\hline Oscinicita Wheeler, 2007 & Unplaced & Unplaced & Unplaced & Unplaced & Unplaced \\
\hline Oscinimorpha Lioy, 1864 & $\begin{array}{l}\text { Aphanotrigonum (Oscinellini } \\
\text { s.l.) }\end{array}$ & $\begin{array}{l}\text { Aphanotrigonum (Oscinellini } \\
\text { s.l.) }\end{array}$ & Oscinellini & Oscinellini & Unplaced \\
\hline Oscinisoma Lioy, 1864 & Eribolus (Oscinellini s.I.) & Unplaced & Oscinisomatini & Oscinisomatini & Unplaced \\
\hline $\begin{array}{l}\text { Paraapallates Cherian in Cherian \& } \\
\text { Tilak, } 2008\end{array}$ & Unplaced & Unplaced & Unplaced & Incertellini & Unplaced \\
\hline $\begin{array}{l}\text { Paracalamoncosis Riccardi \& Bazyar, } \\
\text { 2018* }\end{array}$ & Unplaced & Unplaced & Unplaced & Unplaced & Unplaced \\
\hline Parameijerella Cherian, 1991 & Unplaced & Unplaced & Unplaced & Incertellini & Unplaced \\
\hline Parasiphonella Enderlein, 1913 & Unplaced & Unplaced & Unplaced & Unplaced & Unplaced \\
\hline
\end{tabular}




\begin{tabular}{|c|c|c|c|c|c|}
\hline Platyina Malloch, 1927 & Unplaced & Unplaced & Unplaced & Unplaced & Unplaced \\
\hline Polyodaspis Duda, 1933 & Polyodaspis (Oscinellini s.I.) & Polyodaspis (Oscinellini s.I.) & Fiebrigellini & Fiebrigellini & Unplaced \\
\hline Pselaphia Becker, 1911 & Unplaced? & Unplaced & Botanobiini & Botanobiini & Unplaced \\
\hline Pseudeurina Meijere, 1904 & Lipara (Oscinellini s.I.) & Lipara (Oscinellini s.I.) & Siphonellini & Liparaini & Unplaced \\
\hline Pseudogampsocera Sabrosky, 1964 & Unplaced & Unplaced & Unplaced & Unplaced & Unplaced \\
\hline Pseudogaurax Malloch, 1915 & Gaurax (Oscinellini s.I.) & Gaurax (Oscinellini s.I.) & Botanobiini & Botanobiini & Unplaced \\
\hline Pseudonomba Cherian, 1989 & Unplaced & Unplaced & Unplaced & Rhodesiellini & Unplaced \\
\hline Pseudotricimba Ismay, 1993 & Unplaced & Unplaced & Unplaced & Tricimbini & Unplaced \\
\hline Psilacrum Becker, 1912 & Unplaced & Unplaced & Stenoscinini & Stenoscinini & Unplaced \\
\hline Pterogaurax Duda, 1930 & Unplaced & Unplaced & Unplaced & Botanobiini & Unplaced \\
\hline Rhodesiella Adams, 1905 & Rhodesiellini s.I. & Rhodesiellini s.l. & Rhodesiellini & Rhodesiellini & Unplaced \\
\hline Rhopalopterum Duda, 1929 & Unplaced & Unplaced & Oscinisomatini & Oscinisomatini & Unplaced \\
\hline Sabroskyina Beschovski, 1987 & Unplaced & Unplaced & Unplaced & Oscinisomatini & Unplaced \\
\hline Sacatonia Sabrosky, 1967 & Unplaced & Unplaced & Unplaced & Unplaced & Unplaced \\
\hline Scoliophthalmus Becker, 1903 & Rhodesiellini s.l. & Rhodesiellini s.l. & Scoliophthalmini & Scoliophthalmini & Unplaced \\
\hline Sepsidoscinis Hendel, 1914 & Elachiptera (Oscinellini s.I.) & Myrmecosepsis s.l. & Elachipterini & Elachipterini & Elachipterini \\
\hline Siphonella Macquart, 1835 & Lipara (Oscinellini s.I.) & Siphonella (Oscinellini s.I.) & Siphonellini & Siphonellini & Unplaced \\
\hline Siphonellomyia Seguy, 1934 & Unplaced & Unplaced & Unplaced & Unplaced & Unplaced \\
\hline Siphunculina Rondani, 1856 & $\begin{array}{l}\text { Aphanotrigonum (Oscinellini } \\
\text { s.l.) }\end{array}$ & $\begin{array}{l}\text { Aphanotrigonum (Oscinellini } \\
\text { s.l.) }\end{array}$ & Tricimbini & Tricimbini & Unplaced \\
\hline Speccafrons Sabrosky, 1980 & Unplaced & Siphonella (Oscinellini s.I.) & Incertellini & Siphonellini & Unplaced \\
\hline Stenoscinis Malloch, 1918 & Unplaced & Unplaced (Oscinellini s.I.) & Stenoscinini & Stenoscinini & Unplaced \\
\hline Strandimyia Duda, 1930 & Unplaced & Unplaced & Unplaced & Unplaced & Unplaced \\
\hline Tachinoceros Meijere, 1921 & Rhodesiellini s.I. & Rhodesiellini s.I. & Rhodesiellini & Rhodesiellini & Unplaced \\
\hline Thyridula Becker, 1911 & Unplaced & Unplaced & Unplaced & Unplaced & Unplaced \\
\hline
\end{tabular}




\begin{tabular}{|c|c|c|c|c|c|}
\hline Togeciphus Nishijima, 1955 & Unplaced & Elachiptera (Oscinellini s.I.) & Elachipterini & Elachipterini & $\begin{array}{l}\text { Elachipterini } \\
\text { (Synonimyzed) }\end{array}$ \\
\hline Trachysiphonella Enderlein, 1936 & $\begin{array}{l}\text { Aphanotrigonum (Oscinellini } \\
\text { s.l.) }\end{array}$ & Unplaced & Unplaced & Unplaced & Unplaced \\
\hline Tricimba Lioy, 1864 & $\begin{array}{l}\text { Aphanotrigonum (Oscinellini } \\
\text { s.l.) }\end{array}$ & $\begin{array}{l}\text { Aphanotrigonum (Oscinellini } \\
\text { s.l.) }\end{array}$ & Tricimbini & Tricimbini & Unplaced \\
\hline Tricimbomyia Cherian, 1989 & Unplaced & Unplaced & Unplaced & Tricimbini & Unplaced \\
\hline Tropidoscinis Enderlein, 1911 & Oscinella (Oscinellini s.I.) & Oscinella (Oscinellini s.I.) & Unplaced & Unplaced & Unplaced \\
\hline Tylopterna Bezzi, 1916 & Unplaced & Unplaced & Unplaced & Unplaced & Unplaced \\
\hline Vanchium Cherian, 1999 & Unplaced & Unplaced & Unplaced & Unplaced & Unplaced \\
\hline
\end{tabular}


Appendix II. List and distribution of the genera of Oscinellinae of the world. Caption: NEO, Neotropical; NEA, Nearctic; PAL, Palaearctic; AFR, Afrotropical; ORI, Oriental; AUS, Australian. The * indicates the place of the type-species of each genus. Green background indicates the distribution of the genus.

\begin{tabular}{|c|c|c|c|c|c|c|c|}
\hline & Genus & NEO & NEA & PAL & AFR & ORI & AUS \\
\hline 1 & Acanthopeltastes Enderlein, 1911 & * & & & & & \\
\hline 2 & Agrophaspidium Wheeler \& Mlynarek, 2008 & * & & & & & \\
\hline 3 & Allomedeia Mlynarek \& Wheeler, 2010 & & & & * & & \\
\hline 4 & Alombus Becker, 1914 & & & & $*$ & & \\
\hline 5 & Amnonella Cherian, 1992 & & & & & $*$ & \\
\hline 6 & Anacamptoneurum Becker, 1903 & & & $*$ & & & \\
\hline 7 & Anatrichus Loew, 1860 & & & & $*$ & & \\
\hline 8 & Anomoeoceros Lamb, 1918 & & & & * & & \\
\hline 9 & Apallates Sabrosky, 1980 & & $*$ & & & & \\
\hline 10 & Aphanotrigonella Nartshuk, 1964 & & & $*$ & & & \\
\hline 11 & Aphanotrigonum Duda, 1932 & & & $*$ & & & \\
\hline 12 & Aprometopis Becker, 1910 & & & & $*$ & & \\
\hline 13 & Apterosepsis Richards, 1962 & & & & $*$ & & \\
\hline 14 & Arcuator Sabrosky, 1985 & & & & $*$ & & \\
\hline 15 & Aulacogaurax Becker \& Meijere, 1913 & & & & & * & \\
\hline 16 & Batrachomyia Krefft, 1864 & & & & & & $*$ \\
\hline 17 & Benjaminella Malloch, 1925 & & & & & & * \\
\hline 18 & Bharathella Cherian, 1990 & & & & & $*$ & \\
\hline 19 & Biorbitella Sabrosky, 1980 & & $*$ & & & . & \\
\hline 20 & Cadrema Walker, 1859 & & & & & * & \\
\hline 21 & Calamoncosis Enderlein, 1911 & & & $*$ & & & \\
\hline 22 & Camptopeltes Wheeler \& Mlynarek, 2010 & * & & & & & \\
\hline 23 & Camptoscinella Sabrosky, 1951 & & & & * & & \\
\hline 24 & Cauloscinis Yang \& Yang, 1991 & & & & & $*$ & \\
\hline 25 & Caviceps Malloch, 1924 & & & & & & * \\
\hline 26 & Cestoplectus Lamb, 1918 & & & & & $*$ & \\
\hline 27 & Chaethippus Duda, 1930 & * & & & & & \\
\hline 28 & Chaetochlorops Malloch, 1914 & & $*$ & & & & \\
\hline 29 & Conioscinella Duda, 1929 & * & & & & & \\
\hline 30 & Coryphisoptron Enderlein, 1911 & * & & & & & \\
\hline 31 & Cyrtomomyia Becker, 1913 & & & & * & & \\
\hline 32 & Dactylothyrea Meijere, 1910 & & & & & * & \\
\hline 33 & Dasyopa Malloch, 1918 & & $*$ & & & & \\
\hline 34 & Deltastoma Malloch, 1924 & & & & & & * \\
\hline 35 & Dicraeus Loew, 1873 & & & $*$ & & & \\
\hline 36 & Disciphus Becker, 1911 & & & & & * & \\
\hline 37 & Discogastrella Enderlein, 1911 & * & & & & & \\
\hline 38 & Dysartia Sabrosky, 1991 & & * & & & & \\
\hline 39 & Elachiptera Macquart, 1835 & & & * & & & \\
\hline 40 & Elaphaspis Bezzi, 1912 & & & & * & & \\
\hline 41 & Enderleiniella Becker, 1912 & * & & & & & \\
\hline 42 & Epimadiza Becker, 1910 & & & & $*$ & & \\
\hline 43 & Eribolus Becker, 1910 & & & $*$ & & & \\
\hline 44 & Eugaurax Malloch, 1913 & & * & & & & \\
\hline 45 & Euthyridium Frey, 1923 & & & & & $*$ & \\
\hline 46 & Fiebrigella Duda, 1921 & * & & & & & \\
\hline 47 & Gampsocera Schiner, 1862 & & & & & & * \\
\hline
\end{tabular}




\begin{tabular}{|c|c|c|c|c|c|c|c|}
\hline & Genus & NEO & NEA & PAL & AFR & ORI & AUS \\
\hline 48 & Gaurax Loew, 1863 & & * & & & & \\
\hline 49 & Goniaspis Duda, 1930 & * & & & & & \\
\hline 50 & Hapleginella Duda, 1933 & & & $*$ & & & \\
\hline 51 & Heteroscinis Lamb, 1918 & & & & * & & \\
\hline 52 & Heteroscinoides Cherian, 1989 & & & & & * & \\
\hline 53 & Hippelates Loew, 1863 & & $*$ & & & & \\
\hline 54 & Incertella Sabrosky, 1980 & & $*$ & & & & \\
\hline 55 & Indometopis Cherian, 1989 & & & & & * & \\
\hline 56 & Indonella Cherian in Cherian \& Shinimol, 2008 & & & & & * & \\
\hline 57 & Indophthalmus Cherian, 2002 & & & & & $*$ & \\
\hline 58 & Javanoscinis Andersson, 1977 & & & & & * & \\
\hline 59 & Kurumemyia Kanmiya, 1983 & & & * & & & \\
\hline 60 & Kwarea Sabrosky, 1954 & & & & $*$ & & \\
\hline 61 & Lasiambia Anonymous & & & $*$ & & & \\
\hline 62 & Lasiochaeta Corti, 1909 & & & * & & & \\
\hline 63 & Leucochaeta Becker, 1913 & & & $*$ & & & \\
\hline 64 & Liohippelates Duda, 1929 & & * & & & & \\
\hline 65 & Lioscinella Duda, 1929 & $*$ & & & & & \\
\hline 66 & Lipara Meigen, 1830 & & & $*$ & & & \\
\hline 67 & Loxobathmis Enderlein, 1911 & $*$ & & & & & \\
\hline 68 & Malloewia Sabrosky, 1980 & & * & & & & \\
\hline 69 & Medeventor Wheeler, 2007 & * & & & & & \\
\hline 70 & Meijerella Sabrosky, 1976 & & & & & * & \\
\hline 71 & Melanochaetomyia Cherian, 2002 & & & & & * & \\
\hline 72 & Merobates Duda, 1934 & & & & & $*$ & \\
\hline 73 & Merodonta Malloch, 1940 & & & & & & $*$ \\
\hline 74 & Metasiphonella Duda, 1930 & * & & & & & \\
\hline 75 & Microcercis Beschovski, 1978 & & & * & & & \\
\hline 76 & Mimosepsis Sabrosky, 1951 & & & & $*$ & & \\
\hline 77 & Monochaetoscinella Duda, 1930 & $*$ & & & & & \\
\hline 78 & Neolcella Cherian, 1996 & & & & & $*$ & \\
\hline 79 & Neorhodesiella Cherian, 2002 & & & & & * & \\
\hline 80 & Neoscinella Sabrosky, 1980 & & $*$ & & & & \\
\hline 81 & Nomba Walker, 1860 & & & & & * & \\
\hline 82 & Notaulacella Enderlein, 1911 & * & & & & & \\
\hline 83 & Olcella Enderlein, 1911 & * & & & & & \\
\hline 84 & Onychaspidium Enderlein, 1911 & * & & & & & \\
\hline 85 & Opetiophora Loew, 1872 & & $*$ & & & & \\
\hline 86 & Oscinella Becker, 1909 & & & * & & & \\
\hline 87 & Oscinicita Wheeler, 2007 & * & & & & & \\
\hline 88 & Oscinimorpha Lioy, 1864 & & & * & & & \\
\hline 89 & Oscinisoma Lioy, 1864 & & & * & & & \\
\hline 90 & Paraapallates Cherian in Cherian \& Tilak, 2008 & & & & & * & \\
\hline 91 & Paracalamoncosis Riccardi \& Bazyar, 2018 & $*$ & & & & & \\
\hline 92 & Parameijerella Cherian, 1991 & & & & & * & \\
\hline 93 & Parasiphonella Enderlein, 1913 & * & & & & & \\
\hline 94 & Platyina Malloch, 1927 & & & & & & $*$ \\
\hline 95 & Polyodaspis Duda, 1933 & & & $*$ & & & \\
\hline 96 & Pselaphia Becker, 1911 & & & & * & & \\
\hline 97 & Pseudeurina Meijere, 1904 & & & & & * & \\
\hline 98 & Pseudogampsocera Sabrosky, 1964 & & & & $*$ & & \\
\hline
\end{tabular}




\begin{tabular}{|c|c|c|c|c|c|c|c|}
\hline & Genus & NEO & NEA & PAL & AFR & ORI & AUS \\
\hline 99 & Pseudogaurax Malloch, 1915 & & * & & & & \\
\hline 100 & Pseudonomba Cherian, 1989 & & & & & * & \\
\hline 101 & Pseudotricimba Ismay, 1993 & & & & & & * \\
\hline 102 & Psilacrum Becker, 1912 & & & & * & & \\
\hline 103 & Pterogaurax Duda, 1930 & * & & & & & \\
\hline 104 & Rhodesiella Adams, 1905 & & & & * & & \\
\hline 105 & Rhopalopterum Duda, 1929 & * & & & & & \\
\hline 106 & Sabroskyina Beschovski, 1987 & & & * & & & \\
\hline 107 & Sacatonia Sabrosky, 1967 & & * & & & & \\
\hline 108 & Scoliophthalmus Becker, 1903 & & & $*$ & & & \\
\hline 109 & Sepsidoscinis Hendel, 1914 & & & & & $*$ & \\
\hline 110 & Siphonella Macquart, 1835 & & & * & & & \\
\hline 111 & Siphonellomyia Seguy, 1934 & * & & & & & \\
\hline 112 & Siphunculina Rondani, 1856 & & & * & & & \\
\hline 113 & Speccafrons Sabrosky, 1980 & & * & & & & \\
\hline 114 & Stenoscinis Malloch, 1918 & & * & & & & \\
\hline 115 & Strandimyia Duda, 1930 & * & & & & & \\
\hline 116 & Tachinoceros Meijere, 1921 & & & & & $*$ & \\
\hline 117 & Thyridula Becker, 1911 & & & & & & * \\
\hline 118 & Togeciphus Nishijima, 1955 & & & & & * & \\
\hline 119 & Trachysiphonella Enderlein, 1936 & & & * & & & \\
\hline 120 & Tricimba Lioy, 1864 & & & $*$ & & & \\
\hline 121 & Tricimbomyia Cherian, 1989 & & & & & $*$ & \\
\hline 122 & Tropidoscinis Enderlein, 1911 & * & & & & & \\
\hline 123 & Tylopterna Bezzi, 1916 & & & & & $*$ & \\
\hline 124 & Vanchium Cherian, 1999 & & & & & * & \\
\hline
\end{tabular}


Appendix III. List of species examined in this study, with label details. * = type-species of the genus; ${ }^{* *}=$ material examined only by photo. For institutional abbreviations, see above. $\mathrm{m}$, male; f, female.

\section{Carnidae}

Meoneura flavifacies Collin 1930, $1910^{\prime \prime}$

- Sweden, Lapland, N68.20\%/E18.52 ${ }^{\circ}$, Pan trap, M.V. Tschirnhaus coll., 13-17.xii.1991 (MVTPC)

Milichiidae

Madiza glabra* Fallén, 1820, 10

- Morroco, Marrakech, Imlil S'Asni, C.F. Kassebeer coll., 26.iv.1994 (MVTPC)

Phyllomyza flavitarsis (Meigen 1830), 10"

- Germany, Eifel mountains, Kreis Daun, Malaise trap, K. Colln \& T. Pompé colls., 1.i-31.xii.1991 (MVTPC)

Chloropidae (Siphonellopsinae)

Apotropina brunnipennis (de Meijere 1913), $10^{\circ}$

- Australia, Taree, Lorien Wildlife Refugee, $\mathrm{S} 31^{\circ} 45^{\prime} 04^{\prime \prime} / \mathrm{E} 152^{\circ} 32^{\prime} 03^{\prime \prime}$, Malaise trap, G. \& B. Williams colls., 14-31.xii.2011 (NSW)

Apotropina sp. nov. Hendel 1907, $1910^{x}$

- Australia, Queensland, Carnarvon National Park, M.V. Tschirnhaus coll., 10.x.2002 (MVTPC)

Siphonellopsis lacteibasis Strobl 1906, $1910^{7}$

- Australia, Royal National Park, Aanol G. Daniels coll., 10.iv.1971 (QM)

- Australia, Newnes Tonnel, Aanol G. Daniels coll., 10.iv.1971 (QM)

Chloropidae (Chloropinae)

Chloropella bipartita* malloch $1925,10^{*}$

- $\quad$ Australia, EUA, Near EccLeston, K.A. Spencer coll., 13.xii.1962 (NSW)

Chlorops pumilionis* (Bjerkander 1778), $10^{*}$

- Austria, Linz, Bruno Wahl coll., ? (USNM)

Chloropsina citrivora (Sabrosky 1976), 10

- USA, Hawaii, Maunawili, Bait trap, P. Gow coll., 1.xi.1951 (USNM)

Chloropsina sp.3 nov. Becker 1911, $10^{7}$

- Australia, M.V. Tschirnhaus coll., ? (MVTPC)

Diplotoxa versicolor* (Loew 1863), $10^{*}$

- Canada, Saskatoon, Kennet M. King coll., 18.vi.1925 (MZUSP)

Ectecephala albistylum* Macquart 1851, $10^{x}$

- El Salvador, Santiago de Maria, G.F. \& S. Hevel colls., 29.viii.1972 (MZUSP)

Lasiosina sp.3 Becker 1910, $10^{\pi}$

- $\quad$ USA, Arizona, S. Fork, Cave Creek Coch Co., G. R. Balmer coll., 20.viii.1965 (CDFA) 
Meromyza saltatrix* (Linnaeus 1761), $10^{\prime \prime}$

- Austria, Obergurgl, Tirol 1950m, J.R. Vockeroth coll., 13.viii.1953 (MZUSP)

Meromyzella capensis* Loew 1860, 19

- $\quad$ Nigeria, Zaria, J.C. Deeming coll., 16.v.1970 (MZUSP)

Sagareocerus aberrans* (Schiner, 1868), 10"

- Colombia, Venadillo, Malaise trap, M. Revelo coll., 3.ix.1959 (MZUSP)

Thaumatomyia gemina (Becker 1912), $10^{\prime \prime}$

- Brazil, PR, Chapecó, S2707'11.74'/W52³2'20.22”, M. Savaris \& S. Lampert colls., 1-31.xii.2012 (MZUSP)

Trigonomma coeruleifrons (Becker 1912), 10

- Brazil, SP, Gália, Sweeping, ?, 3.ix.2010 (MZUSP)

Chloropidae (Rhodesiellinae)

Psilacrum lineatum Riccardi \& Ismay 2017, $1910^{\circ}$

- $\quad$ Brazil, RS, Camaquã, S31.12159\%/W51.79237², Malaise trap, F.D. Kirst \& R.F. Krüger coll., 25.xi.2011 (MZUSP)

Rhodesiella sp.1 Adams 1905, 19

- $\quad$ Germany, Rhein near Krefeld, Malaise trap, M.V. Tschinrhaus coll., v.1990 (MVTPC)

Rhodesiella sp.2 Adams 1905, 10

- Cameroon, Yaoundé, Malaise trap, Sevilor Kekeumou coll., 14-31.v.2010 (JFPC)

Rhodesiella sp.3 Adams 1905, 10"

- $\quad$ Ivory Coast, S. Hilger \& C.F.Kassebeer colls., 13.i-24.ii.1998 (MZUSP)

Stenoscinis sp. Malloch 1918, $10^{x}$

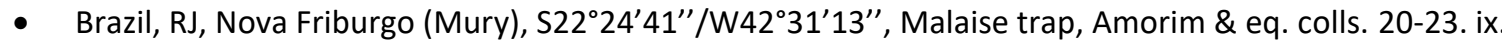
2015 (DMEL)

Chloropidae (Oscinellinae)

Anatrichus erinaceus* Loew 1860, 29

- Sumatra, Fort de Kock, E. Jacobson coll., 1925 (ZMHU)

Apallates dissidens* (Tucker 1908), $2910^{\pi}$

- Bahamas, Grand Bahama Island, W. E. Steiner, M. J. \& R. Molineaux colls., 24.v.1987 (USNM)

- Maryland, Calvert Co. Chesapeake Beach, Sweeping, Jos. J. Anderson coll., 7.vi.1981 (USNM)

- USA, Florida, ? coll., 6.iii.1930 (USNM)

Aphanotrigonum trilineatus* (Meigen 1830), 1910

- $\quad$ Poland, Nimptsch Schles, Duda coll., 26.iv.1912 (ZMHU)

Batrachomyia nigritarsis* Skuse 1889, $1910^{\prime \prime}$

- $\quad$ Australia, From Frog Royal Park, D.K. McAlpine, D.S. Kent \& B. Day colls., 30.vi.1980 (NSW)

Biorbitella hesperia* (Sabrosky 1940), 19 10

- $\quad$ USA, Antioch, Dickson coll., 18.x.1936 (USNM)

- $\quad$ USA, Victorville, San Berna, R. Schuster \& G. A. Marsh colls., 2.v.1953 (USNM) 
Cadrema pallida (de Meijere 1904), $1910^{7}$

- Madagascar, $\mathrm{N} 14^{\circ} 38^{\prime} / \mathrm{E} 50^{\circ} 12^{\prime}$. M.V Tschirnhaus coll., 6.vi. 2006 (MVTPC)

Calamoncosis minima (Strobl 1893), $1910^{\pi}$

- Germany, emerged from Lipara lucens galls, Kiel-Wellsee coll., 15.iv.1976 (MVTPC)

Chaethippus sp. Duda 1930, 19

- Peru, Madre de Dios, S13.14\% W69.61', Malaise trap, B.Brown \& G.Kung 23-25.vii.2001 (MZUSP)

Chaetochlorops inquilina* Coquillett (orig. des.). Ref. Sabrosky 1950c (key, biol.), $1910^{\text {* }}$

- USA, Falls Church Va, N!, JJDeGryse. coll., 10-19.viii.? (USNM)

- USA, Glassboro, N. J., W.F. Rapp, Jr. coll., 20.viii.1943, (NHM)

Conioscinella soluta* (Becker 1912), $1930^{\pi}$

- Costa Rica, Farm La Caja., S. José coll., 12.vi-20.vii.1924 (ZMHU)

- Brazil, Nova Teutônia, B27 ${ }^{\circ} 11^{\prime} /$ L52 $^{\circ} 23^{\prime}$, Fritz Plaumann museum coll., 1.iv.1937 (NHMUK) (Conioscinella soluta var. rufoscutellata)

Deltastoma unipunctatum Malloch 1924, 19

- Australia, Lorien Wildlife Refuge, 3 km N. Lansdowne, G.Williams coll., 3.xi.1991 (NSW)

Dicraeus vagans Meigen 1838, 1 우 $10^{\pi}$

- Germany, Neumorscheu, Halberg, N5103'/E90³6', M.V. Tschirnhaus coll., 16.vi.2007 (MVTPC)

Disciphus peregrinus* Becker 1911, $1910^{7}$

- Sumatra, SumatraZool, E. Jacobson coll., 1925 (ZMHU)

Elachiptera bimaculata (Loew 1845), $1910^{\circ}$

- Iran, Fars, Jahrom, Malaise trap, Z. Bazyar coll., 2010 (ZBPC)

Elachiptera brevipennis* (Meigen 1830), $1910^{7}$

- Poland, Laband, Os. L. Duda coll., 2.ix.1935 (ZMHU)

- Poland, Nimptsch, Schles. \& Duda colls., 01.v.1951 (AMHU)

Epimadiza sp. Becker 1910, 1 우

- Kenya, Kakamega Forest, Kramer et al. colls., 2.v.1929 (MVTPC)

Eribolus hungaricus Becker 1910, 1 웅 $10^{7}$

- Germany, Danish border, M.V. Tschirnhaus coll., 12.vi.1968 (MVTPC)

Eugaurax sp. Malloch 1913, $10^{\text {* }}$

- Nicaragua, Jinotega, Penas Blancas, N13.17\%/W35.38, Maes \& B. Hernandez colls., 25.vii.1997 (MZUSP)

Fiebrigella sp. Duda 1921, $10^{7}$

- Brazil, MA, Bom Jardim REBIO-RES, Armadilha Suspensa, F. Limeira-de-Oliveira, J.T.Camara \& J.O.A. Silva colls., 17-27. i.2010 (INPA)

Gaurax festivus* Loew 1863, $10^{7}$

- Maryland, Maryland Bethesda, McPhail Trap, G.C. Steyskal coll., 1.vii.1978 (USNM)

Goniaspis truncata (Malloch 1913), 19

- Mexico, Tepec, R. \& K. Dreisbach coll., 7.v.1956 (USNM) 
Hapleginella laevifrons* (Loew 1858), $1910^{*}$

- Austria, Tyrol. Oetz., J. Abraham \& J.C. Deeming Colls., 14-19.vi.1964 (NHM)

- Azerbaijan, Zabrat, D. Duda coll., 16.vi.1931 (ZMHU)

Heteroscinis hystrix Seguy, 1957, $1910^{7}$

- Sierra Leone, extract from rotting coffee berries, E. Hargreaves coll., 17.x.1936 (NHM)

- Nigeria, C.I.E. coll., 5-v-1983 (NHM)

Hippelates plebejus* Loew 1863, $1910^{*}$

- USA, Gatlinburg. Tenn, Sweeping, R.H. Whittaker coll., 29.v.1947 (USNM)

- USA, Ithaca N.Y., R.C.Shannon coll., 5.ix.1922 (USNM)

Incertella incerta* (Becker 1912), $2910^{*}$

- Canada, Ungva Bay, J.F. McAlpine coll., 29.vii.1954 (USNM)

- USA, Union Cty. Tn., G.I. Reeves coll., ? (USNM)

- Canada, Treesbank, Man, N. Criddle coll., 7.ix.1915 (USNM)

Lasiambia palposa (Fallén 1820), $1910^{\pi}$

- Italy, $\mathrm{N} 46^{\circ} 37^{\prime} / \mathrm{E}^{\circ} 0^{\circ} 35^{\prime}$, Ziegler et al. colls., ? (MVTPC)

Lioscinella sulfurihalterata* (Enderlein 1911), 19

- Paraguay, San Bernadino, K.Fiebrig coll., 3.ii.? (ZMHU)

Lipara lucens* Meigen 1830, 2 ?

- Germany, Berlin, Lichtwardt coll., 3.vi.1911 (ZMHU)

Lipara pullitarsis Doskocil \& Chvála 1971, 19 10"

- Germany, Bielefeld, extract from Phragmites, Doskocil \& Chvala colls., 1977 (MVTPC)

Loxobathmis obliquans* Enderlein 1911, 1 i $10^{*}$

- USA, San Mateo, Pablo Schild coll., ? (ZMHU)

Malloewia diabolus* (Becker 1912), 19 10 $10^{x}$

- USA, Redding Calif., B. Wade coll., 11. iv.1955 (USNM)

Medeventor nubosus* Wheeler 2007, $10^{* * *}$

- Costa Rica, Puntarenas, Light trap, B.J.Sinclair coll., 22-27.ii.1991 (CNC)

Medeventor tschirnhausi Bazyar \& Silva 2018, $1910^{7}$

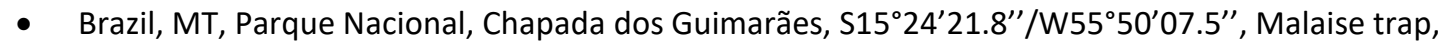
Lams \& Nihei colls., 27.viii-11.ix.2011 (MZUSP)

Metasiphonella sp. Duda 1930, $10^{*}$

- Brazil, SP, Ribeirão Preto, S2109'54"/W4750'56", Sweeping, P. Riccardi coll., 20.xi.2016 (MZUSP)

Microcercis albipalpis (Meigen 1830), $1910^{\pi}$

- Germany, Hessen : Neumorschen, M.V. Tschirnhaus coll., 16.vi.2007 (MVTPC)

Monochaetoscinella anonyma* (Williston 1896), $1910^{7}$

- USA, Hawaii, near shore, G. Steyskal coll., 6.vi.1968 (USNM)

Neoscinella gigas* (Sabrosky 1940), 19

- USA, lowa, Gould Warren coll., 5.vi.1935 (USNM) 
Notaulacella sp. Enderlein, 1911, $10^{7}$

- Nicaragua, Masaya Las Flores, UV trap, Lecocq \& Cantamessa colls., vi.1993 (DMEL)

Opetiophora straminea* Loew 1872, $1910^{*}$

- USA, Moab, Utab, G.F.Knowlton coll., 3. viii.1942 (USNM)

Oscinella frit* (Linnaeus 1758), $1910^{*}$

- USA, Hagerstown, Md, Reared from wheat, PRMyers coll., 13.x.1914 (USNM)

- USA, Middleburg Pa, Reared from wheat, PRMyers coll., 5.xi.1919 (USNM)

Oscinella cf. flavitibia (Duda 1933), 10

- Brazil, Sta. Rita, Sweeping, Amorim \& Falaschi colls., 30.xi.2008 (DMEL)

Oscinimorpha albisetosa (Duda 1932), $1910^{\circ}$

- Germany, Island Mellum North Sea, Pan trap, V. Haeseler coll., 8.vi.1985 (MVTPC)

Oscinisoma cognatum (Meigen 1830), $1910^{\pi}$

- Germany, Kiel, Extract from Glyceria maxima, H. Meyer coll., 26.vi.1983 (MVTPC)

Paracalamoncosis brasiliensis* Riccardi \& Bazyar 2018, 10"

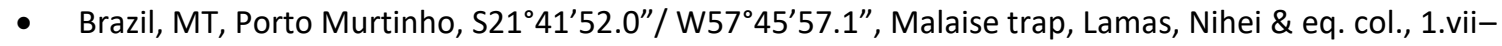
15.viii.2012 (MZUSP)

Polyodaspis ruficornis* (Macquart, 1835), $1910^{\pi}$

- Greece, Samothrace, Guichard \& Harvey coll., 17.viii. 1962 (NHM)

- France, Abzac (chaseute), K.A. Spencer coll., xi.1989 (NHM)

Pseudeurina maculata* de Meijere 1904, $10^{*}$

- Malaysia, Malaysia Johore, 15K NE Kata, J.W. Ismay coll., 28.x.1983 (JIPC)

Pseudeurina sp. nov., $1910^{\pi}$

- Brazil, SP, Salesópolis, S2339.06 $/ \mathrm{W} 45^{\circ} 53.53^{\prime}$, Sweeping, P.R. Riccardi coll., 20.xi.2016 (DMEL)

Pseudogaurax anchora* (Loew 1866), $1910^{*}$

- USA, Falls Church, Reared from Philosamia, A.B.Gurney coll., x.1950 (USNM)

- USA, Lexington, Hatched from Bagworm, KY, Monte Johnson coll., 16.v.1992 (USNM)

Rhopalopterum limitata* (Becker 1912), $1920^{\circ}$

- Costa Rica, San Mateo CR, Pablo Schild coll., ? (USNM)

- Colombia, Cundin Amarca, E.I.Schlinger \& E.S.Ross colls., 9.iii.1955 (USNM)

- Costa Rica, K.A. Spencer coll., 25.i1982 (NHM)

Sepsidoscinis maculipennis* Hendel 1914, 1 우 10"

- $\quad$ Sumatra, Fort de Kock, E. Jacobson coll., 1923 (ZMHU)

Siphonella oscinina* (Fallén 1820), $2910^{*}$

- USA, Woods Hole, A.H.Sturtevant coll., ix.1970 (USNM)

- USA, Shannon Camp, C. Beegle coll., 15.viii.1967 (USNM)

- Norway, Aurland, M.W. Joyce coll., 3.ix.1958 (NHM)

Siphunculina brevinervis* Rondani 1856, 1 i $10^{\prime \prime}$

- Poland, Nimptsch, Schles Duda coll., 8.ix.1910 (ZMHU)

- $\quad$ Austria, Rauden, O.S.I. Duda coll., 2.viii.1932 (ZMHU) 
Speccafrons mallochi* Sabrosky 1938, $1910^{\pi}$

- USA, Washington DC, From bird nest, RC SHANNON coll. 2.ii.1913 (USNM)

Strandimyia sp. Duda 1930, $10^{7}$

- Brazil, Nova Teulonia, B27 $11^{\prime} / L 52^{\circ} 23^{\prime}$, Fritz Plaumann Museum coll., x.1970 (MZUSP)

Trachysiphonella pygmaea (Meigen 1838), $1910^{\pi}$

- Germany, Niederbeisheim, N51 $03^{\prime} /$ E9 $^{\circ} 31^{\prime}$, M.V. Tschirnhaus coll., 16.vi.2007 (MVTPC)

Tricimba lineella* (Fallén 1820), $1910^{*}$

- Germany, Oldenburg, Schildhorn coll., v.1998 (USNM)

- UK, Bristol, E.A. Fonseca coll., 15.ix.1978 (NHM) 